Technische Universität Berlin

Fakultät Verkehrs- und Maschinensysteme

Institut für Land- und Seeverkehr

June Young Lee

geboren am 6. Januar 1969

\title{
Entwurf von Offshore-Strukturen unter Einsatz globaler Optimierungsstrategien
}

\author{
Bei der Fakultät V der Technischen Universität Berlin \\ -Verkehrs- und Maschinensysteme- \\ zur Erlangung des akademischen Grades \\ Doktor der Ingenieurwissenschaften \\ - Dr.-Ing. - \\ genehmigte Dissertation
}

Promotionsausschuss:

Vorsitzender: Prof. Dr.-Ing. Wolfgang Nitsche

Berichter: Prof. Dr.-Ing. Günther F. Clauss

Berichter: Prof. Dr.-Ing. Dr. h.c. Horst Nowacki

Berichter: Dr.--Ing. Lothar Birk

Tag der wissenschaftlichen Aussprache: 20. Feb. 2004

Berlin 2004

D83 


\section{Zusammenfassung}

In der vorliegenden Dissertation wird ein verbessertes numerisches System zum praktischen Entwurf von Offshore-Strukturen vorgestellt. Das System der hydrodynamischen Formoptimierung meerestechnischer Konstruktionen beruht auf drei eigenständigen numerischen Werkzeugen: der Geometriemodellierung (CAD), der hydrodynamischen Analyse (CFD) und der Wahrscheinlichkeitstheorie zur Abschätzung der hydrodynamischen Eigenschaften im unregelmäßigen Seegang. Das Gesamtsystem wird durch Optimierungsalgorithmen gesteuert, um die Seegangseigenschaften in einem automatisierten Prozess zu verbessern. Ausgehend von einem vorgegebenen Entwurf oder zufällig ausgewählter Ausgangsstrukturen werden Formvariationen durchgeführt, bis ein Minimum der Zielfunktion gefunden ist.

Zur Berechnung der Zielfunktionen stehen zwei unterschiedliche Programme zur Verfügung, die abhängig vom Typ der Struktur selektiv eingesetzt werden können: Für die Analyse hydrodynamisch kompakter Strukturen wird das 3D-Diffraktions-Radiations-Programm WAMIT verwendet, womit die zuverlässige Analyse der Kräfte und Bewegungen beliebig geformter Körper im Seegang möglich ist. Für hydrodynamisch transparente Strukturen wird das auf der Basis der vektoriellen Morisongleichung entwickelte Programm TFC (Transfer Function Calculation) eingesetzt, das erfolgreich mit Seegangsversuchsdaten validiert wurde. Damit werden die Übertragungsfunktionen für Kräfte und Bewegungen weitgehend analytisch und dadurch sehr viel schneller ermittelt, als mit einem 3D-Panelverfahren. Da das erweiterte Programm auch die zähigkeitsbedingte Widerstandskraft einbezieht, kann der Einfluss der Zähigkeit auf die Formoptimierung untersucht werden.

Bei der Formoptimierung hydrodynamisch kompakter Strukturen wird zur Vergrößerung der Formenvielfalt ein erweitertes Formgenerierungsverfahren eingesetzt. Aus den Formparametern werden die Grundkurven einer Strukturkomponente definiert und damit die Oberfläche der Komponente durch eine modifizierte Sweeping-Operation in Form einer Non-Uniform-Rational-B-Spline (NURBS) Fläche automatisch erzeugt, ohne jeglichen Eingriff des Benutzers.

Zur Bewertung der Zielfunktion werden die relativen Häufigkeiten der Seegangszustände auf Grundlage der Langzeit-Seegangsstatistik ermittelt, bei denen der operationelle Betrieb unterbrochen werden muss. Die Summation dieser Seegangszustände, d.h. die seegangsbedingte Ausfallzeit, wird als Gütekriterium für die Formoptimierung herangezogen. Außerdem werden auch Ermüdungsschäden als Zielfunktion eingesetzt, um die Lebensdauer ausgewählter Konstruktionselemente zu maximieren. Alle Zielfunktionen werden mittels einer linearen Spektralanalyse im Frequenzbereich durchgeführt.

Globale Optimierungsstrategien wurden mit einer deterministischen Methode (Sequential Quadratic Programming) hinsichtlich des Aufwandes und der Leistungsfähigkeit verglichen. An Beispielen wird illustriert, wie sich exzellente Ausgangsstrukturen mit deterministischen Verfahren noch erheblich verbessern lassen. Im multimodalen Fall kann bei der Anwendung lokaler Algorithmen das beste Optimum verfehlt werden. Hier können globale Optimierungsstrategien mit größerer Wahrscheinlichkeit bessere lokale Minima und eventuell das gesuchte globale Optimum erzielen. 


\begin{abstract}
This thesis presents an improved numerical procedure for optimum adjustment of shapes to environmental conditions and presents new results in the design of offshore structures. By employing parametric shape generation, numerical hydrodynamic analysis and assessment tools as well as Nonlinear Programming algorithms the seakeeping characteristics of offshore structures are improved automatically. Starting from an initial design defined by form parameters or from randomly selected initial designs, free variables of the hull shape are to be modified, until new optimum offshore structures with significant improvement of seakeeping qualities are found within the provided constraints, such as hydrostatic stability at design draft.

Two different Program System are available for the evaluation of objective functions. Depending on the type of structure - hydrodynamically transparent or compact hydrodynamic characteristics are evaluated by the Program TFC (Transfer Function Calculation) based on a Morison-type analysis or the 3-D diffraction-radiation software package WAMIT. Validated successfully by experimental results, the Program TFC can take viscous effects into consideration in the hydrodynamic shape optimization.

For the shape optimization of hydrodynamically compact structures the extended surface generation procedure is applied to enhance the diversity of shape variations. Based on the selected form parameters basic lines of structure components are defined and a NURBS surface of the hull surface is generated by the modified sweeping operation without any user interaction.

Rational design criteria based on short and long-term wave statistics are introduced and utilized as objective function in the optimization process. As a result, significant forces, motions, downtime and fatigue damage due to irregular sea states are minimized. Performance of a deterministic optimization algorithm (Sequential Quadratic Programming) is compared to stochastic methods like Simulated Annealing and Genetic Algorithms or combined algorithms (hybrid method). It is illustrated that favourable initial designs can be significantly improved by local methods. In the multimodal case local methods cannot ensure the best possible design. Here global optimization strategies may find better solution with greater probability and in some cases may even find the global optimum.
\end{abstract}




\section{Inhaltsverzeichnis}

Abbildungsverzeichnis $\quad$ ix

Tabellenverzeichnis $\quad \mathrm{x}$

1 Einleitung 1

2 Stand der Technik 4

3 Hydrodynamische Formoptimierung $\quad 9$

4 Optimierungsstrategien $\quad \mathbf{1 4}$

4.1 Mathematische Formulierung der Optimierungsaufgabe . . . . . . . . . 14

4.2 Klassifizierung der Optimierungsalgorithmen . . . . . . . . . . . . . . . 15

4.3 Lokale Optimierungsstrategien . . . . . . . . . . . . . . . . . . 17

4.3.1 Tangentensuchmethode $(\mathrm{TSM}) \ldots \ldots \ldots . . \ldots 17$

4.3.2 Sequential Quadratic Programming (SQP) . . . . . . . . . 17

4.4 Globale Optimierungsstrategien . . . . . . . . . . . . . . . . . . . . 19

4.4.1 Genetische Algorithmen (GA) . . . . . . . . . . . . . . 20

4.4.2 Simulated Annealing (SA) . . . . . . . . . . . . . . 21

4.4.3 Angepasste Branch-and-Bound Algorithmen (BaB) . . . . . . . 23

4.5 Verhalten bei multimodalen Zielfunktionen . . . . . . . . . . . . . 24

4.5.1 Sequential Quadratic Programming (SQP) . . . . . . . . . . . 24

4.5.2 Adaptive Simulated Annealing (ASA) . . . . . . . . . . . . . 25

4.5.3 Genetische Algorithmen (GA) . . . . . . . . . . . . . 26

4.5.4 Hybride Optimierung . . . . . . . . . . . . . . 27

5 Definition und Berechnung der Zielfunktionen $\quad 29$

5.1 Geometriemodellierung . . . . . . . . . . . . . . . . . . . . . 29

5.2 Hydrodynamische Analyse . . . . . . . . . . . . . . . . . . . 33

5.3 Zielfunktionen . . . . . . . . . . . . . . . . . 35

5.3.1 Spektralanalyse . . . . . . . . . . . . . 36

5.3 .2 Bewertung der Ausfallzeit . . . . . . . . . . . . . . . . . 38

5.3.3 Bewertung von Ermüdungsschäden . . . . . . . . . . . . . . . 40 
6 Globale Optimierungsstrategien im Vergleich $\quad 44$

6.1 Optimierung hydrodynamisch transparenter Strukturen . . . . . . . . . 44

6.1.1 Halbtaucher mit 4 freien Variablen . . . . . . . . . . . . . 45

6.1.2 Halbtaucher mit 9 freien Variablen . . . . . . . . . . . . . . 52

6.1.3 Effekte der Zähigkeit auf die Übertragungsfunktion . . . . . . . 60

6.1.4 Zähigkeitseinfluss auf die Optimierung . . . . . . . . . . 63

6.2 Optimierung hydrodynamisch kompakter Strukturen . . . . . . . . . . 72

6.2.1 Halbtaucher . . . . . . . . . . . . . . . . . . 72

6.2.2 Tension Leg Plattform . . . . . . . . . . . . . . . . . . . . 79

$\begin{array}{llr}7 & \text { Schluss } & 87\end{array}$

$\begin{array}{lr}\text { Literaturverzeichnis } & 89\end{array}$

$\begin{array}{ll}\text { A Darstellung der Spantarealkurve } & 99\end{array}$

$\begin{array}{lr}\text { B Grundlagen des TFC-Programmes } & 102\end{array}$

B.1 Hydrodynamisch transparente Strukturen . . . . . . . . . . . . . . . . . 102

B.2 Beschreibung der Struktur . . . . . . . . . . . . . . . . . . . . . . 102

B.2.1 Koordinatensystem . . . . . . . . . . . . . . . . 103

B.2.2 Bewegungsfreiheitsgrade . . . . . . . . . . . . . . . . 104

B.3 Berechnung der Kräfte auf ein Strukturelement . . . . . . . . . . . . 105

B.3.1 Kraft normal zur Zylinderachse . . . . . . . . . . . . . 105

B.3.2 Kraft in Richtung der Zylinderlängsachse . . . . . . . . . . . . . 114

B.3.3 Hydrostatische Rückstellkraft . . . . . . . . . . . . . . . . . . . 121

B.4 Die äußere Kraft auf die Gesamtstruktur . . . . . . . . . . . . . . . . . 124

B.5 Die Bewegungsdifferentialgleichungen . . . . . . . . . . . . . . . 124

B.6 Die Berechnung der Bewegungsübertragungsfunktion . . . . . . . . . . 125

B.7 Das Programm TFC (Transfer Function Calculation) . . . . . . . . . . 127

B.8 Validierung des Programmes mit Versuchdaten . . . . . . . . . . . . . 129

C Grundlagen des Programmsystems WAMIT 133

C.1 Das Randwertproblem für einen Körper im Wellenfeld . . . . . . . . . . 133

C.2 Numerische Lösung . . . . . . . . . . . . . . . . . . . . . . . . . . . 137

C.3 Erregerkräfte und die Übertragungsfunktion der Bewegung . . . . . . . 138 


\section{Abbildungsverzeichnis}

3.1 Teilaufgaben der hydrodynamischen Formoptimierung im Entwurfsprozess

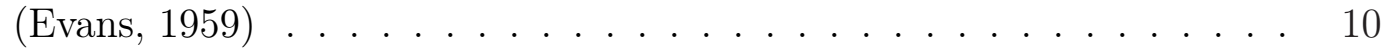

3.2 Traditioneller, interaktiver Rumpfentwurf versus hydrodynamische Formoptimierung (Birk et al., 2002) . . . . . . . . . . . . . . . 11

3.3 Ablauf der hydrodynamischen Formoptimierung (Birk, 1998) . . . . . 12

4.1 Grundsätze der Branch-and-Bound Methoden . . . . . . . . . . . . . . 23

4.2 Eine analytische, multimodale Funktion mit 2 Variablen . . . . . . . 25

4.3 Minimierung einer Griewank-Funktion unter Anwendung des Sequential Quadratic Programming (lokale Suchstrategie). . . . . . . . . . 26

4.4 Minimierung der Griewank-Funktion unter Anwendung von Adaptive Simulated Annealing (ASA, globale Optimierungsstrategie) . . . . . . . 26

4.5 Minimierung der Griewank-Funktion unter Anwendung des Genetischen Algorithmus (GA, globale Optimierungsstrategie) . . . . . . . . . . 27

4.6 Minimierung der Griewank-Funktion unter kombinierter Anwendung von ASA und SQP-Methode (Hybride Optimierungsmethode) . . . . . . . . 28

4.7 Minimierung der Griewank-Funktion: kombinierte Anwendung von GA und SQP-Methode (Hybride Optimierungsmethode) . . . . . . . . . 28

5.1 Formparameter . . . . . . . . . . . . . . . . . . . . 30

5.2 Grundkurven und Parameter der parametrischen Geometrieerzeugung einer Strukturkomponente . . . . . . . . . . . . . . . . . . . . . . . 31

5.3 Zusammenfügen mehrerer Komponenten mit Übergangsflächen (blending patches) am Beispiel eines hydrodynamisch kompakten Halbtauchers 32

5.4 Mathematisches Modell der Zielfunktion . . . . . . . . . . . . . . . . . 35

5.5 Übertragungsfunktion einer meerestechnischen Konstruktion (Clauss et al., 1992) . . . . . . . . . . . . . . . 36

5.6 Absolute Häufigkeit und daraus abgeleitete zweidimensionale Wahrscheinlichkeitsdichteverteilung für ein Jahr (Seegebiet Haltenbanken vor der norwegischen Küste, Mathisen und Bitner-Gregersen, 1990). Beispiel für Grenzkurven der für den Betrieb zulässigen signifikanten Wellenhöhen . 39

5.7 Prinzipielle Darstellung der S-N Kurve . . . . . . . . . . . . . . . . . 41

6.1 Geometrie der Ausgangsstrukturen (A) und (B) . . . . . . . . . . . . 45 
6.2 Optimierungsschritte und Änderung der Zielfunktion für SequentialQuadratic-Programming mit den Ausgangsstrukturen (A) und (B) . . . . 46

6.3 Optimierungsschritte und Änderung der Zielfunktion (a) für Genetischen Algorithmus und (b),(c) für Adaptive Simulated Annealing Al-

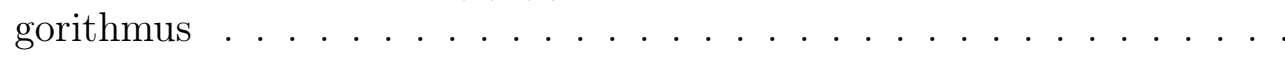

6.4 Entwicklung von Geometrie und Zielfunktion im Laufe der hydrodynamischen Formoptimierung mit einem Genetischen Algorithmus . . . . .

6.5 Darstellung der gefundenen Optima im Zielfunktionsraum für $x_{3}=$ $60 m^{2}$ und $x_{4}=189 m^{2} \ldots \ldots \ldots \ldots$

6.6 Vergleich der Übertragungsfunktionen der Tauchbewegung bei unterschiedlichen Ausgangsstrukturen . . . . . . . . . . . . . . .

6.7 Vergleich der Übertragungsfunktionen der Stampfbewegung bei unterschiedlichen Ausgangsstrukturen . . . . . . . . . . . . . . .

6.8 Vergleich der Geometrien und Grenzkurven der zulässigen signifikanten Wellenhöhen (Optimierung mit Ausgangsstruktur (B)): Seegangsrichtung von vorne . . . . . . . . . . . . . . . . . . . . . . . 52

6.9 Definition der Formparameter für den Halbtaucher mit 9 freien Variablen 53

6.10 Optimierung eines Halbtauchers mit 9 freien Variablen: Ergebnis für die lokale SQP Strategie . . . . . . . . . . . . . . . . . 54

6.11 Optimierungsschritte und Änderung der Zielfunktion für den SQP-Algorithmus: (a) mit der Ausgangsstruktur (A) (b) mit der Ausgangsstruktur (B) (c) mit der Ausgangsstruktur (C . . . . . . . . . . . . . 56

6.12 Verlauf der Optimierung für den BaB Algorithmus: Änderung der Zielfunktion auf Optimierungsschritte (a) Änderung der Zielfunktion auf BaB Zyklen . . . . . . . . . . . . . . . . .

6.13 Optimierungsschritte und Änderung der Zielfunktion für den Genetischen Algorithmus (a) und für den Adaptive Simulated Annealing Algorithmus $(\mathrm{b}) \ldots \ldots \ldots \ldots \ldots$

6.14 Ergebnisse der Optimierung eines Halbtauchers mit 9 freien Variablen mit den globalen Optimierungsalgorithmen (GA und ASA) . . . . . .

6.15 Ergebnisse der Optimierung eines Halbtauchers mit 9 freien Variablen mit den hybriden Optimierungsalgorithmen (GA+SQP und ASA+SQP)

6.16 Vergleich der Geometrien und Grenzkurven der zulässigen signifikanten Wellenhöhen: Optimierungen mit Ausgangsstruktur (C) mit der Begrenzung der maximalen Amplituden auf $2[\mathrm{~m} / \mathrm{m}]$. . . . . . . . . . . .

6.17 Effekt der Zähigkeit auf die Übertragungsfunktion der Tauchbewegung:

(a) Pierson-Moskowitz Spektren für $H_{s}=1.0 m$ und $T_{0}=[12 s, 11 s, \cdots, 5 s, 4 s]$

(b) Übertragungsfunktionen der Tauchbewegung . . . . . . . . . . . . . 61

6.18 Effekt der Zähigkeit auf die Antwortspektren der Tauchbewegung für unterschiedliche Zero-up-crossing Perioden $T_{0} \ldots \ldots \ldots$

6.19 Einfluss der Zähigkeit auf die Übertragungsfunktion der Tauchbewegung in Abhängigkeit der Wellenamplitude $\zeta_{a}$ bei Resonanz- und Auslöschungsfrequenz . . . . . . . . . . . . . . . . . 
6.20 Optimierungsschritte und Änderung der Zielfunktion für den SQP-Algorithmus unter Berücksichtigung der Zähigkeit $C_{d}=1.0, \zeta_{a}=1.0 \mathrm{~m}$ : (a) mit der Ausgangsstruktur (A) (b) mit der Ausgangsstruktur (B) (c) mit der Ausgangsstruktur C . . . . . . . . . . . . . . .

6.21 Geometrie der optimierten Strukturen mit dem lokalen Optimierungsalgorithmus SQP unter Berücksichtigung der Zähigkeit $C_{d}=1.0, \zeta_{a}=1.0 \mathrm{~m} 65$

6.22 Geometrie der optimierten Strukturen mit den globalen Optimierungsalgorithmen unter Berücksichtigung der Zähigkeit $C_{d}=1.0, \zeta_{a}=1.0 \mathrm{~m}$.

6.23 Geometrie der optimierten Strukturen mit den hybriden Optimierungsalgorithmen unter Berücksichtigung der Zähigkeit $C_{d}=1.0, \zeta_{a}=1.0 \mathrm{~m}$.

6.24 Vergleich der Übertragungsfunktionen der Tauchbewegung: Optimierung (a) mit Begrenzung des maximalen RAOs auf $2[\mathrm{~m} / \mathrm{m}]$ (b) mit Berücksichtigung des Zähigkeitseinflusses mit $C_{d}=1.0, \zeta_{a}=1.0 \mathrm{~m}$ (c) mit Berücksichtigung des Zähigkeitseinflusses mit $C_{d}=1.0, \zeta_{a}=5.0 \mathrm{~m}$.

6.25 Vergleich der Antwortspektren der Tauchbewegung für die optimierten Strukturen (ASA+SQP): (a) mit Berücksichtigung des Zähigkeitseinflusses mit $C_{d}=1.0, \zeta_{a}=1.0 \mathrm{~m}(\mathrm{~b})$ mit Berücksichtigung des Zähigkeitseinflusses mit $C_{d}=1.0, \zeta_{a}=5.0 \mathrm{~m} \ldots \ldots \ldots$

6.26 Vergleich der Geometrien und Grenzkurven der zulässigen signifikanten Wellenhöhen: Optimierungen mit Ausgangsstruktur (C) bei Berücksichtigung der Zähigkeit $C_{d}=1.0, \zeta_{a}=1.0 \mathrm{~m} \ldots \ldots \ldots$

6.27 (a) Verteilung der Häufigkeit von Seegangszuständen mit Windrichtung $\beta$ im Nordostatlantik (Marsden Square 182) (b) Abgeleitete 2D Wahrscheinlichkeitsdichteverteilung für die Windrichtung 240 [Grad] . . . . .

6.28 Ergebnis der Optimierung eines hydrodynamisch kompakten Halbtauchers mit dem Sequential Quadratic Programming Algorithmus (SQP)

6.29 Optimierungsschritte und Änderung der Zielfunktion: (a) für den Sequential Quadratic Programming Algorithmus, (b) für den Adaptive Simulated Annealing Algorithmus . . . . . . . . . . . . .

6.30 Entwicklung von Geometrie und Änderung der Zielfunktionswerte im Laufe der hydrodynamischen Formoptimierung mit einem Genetischen Algorithmus für den Halbtaucher mit $\triangle=50,000 m^{3} \ldots$. . . . . . . .

6.31 Ergebnis der globalen Optimierung eines hydrodynamisch kompakten Halbtauchers mit dem Genetischen Algorithmus (GA) und dem Adaptive Simulated Annealing Algorithmus (ASA) . . . . . . . . . .

6.32 Übertragungsfunktionen der Tauchbewegung für die Ausgangsstruktur und die optimierten Strukturen bei Windrichtung $\beta=240$ [Grad] . . .

6.33 Verteilung der zu erwartenden Ausfallzeit nach Windrichtung $\beta$ im Nordostatlantik (Marsden Square 182) . . . . . . . . . . . . .

6.34 Vergleich der Geometrien und Grenzkurven der zulässigen signifikanten Wellenhöhen für die Ausgangsstruktur und die optimierten Strukturen bei Windrichtung $\beta=240$ [Grad] . . . . . . . . . . . . . . .

6.35 Geometrie und Formparameter der Ausgangsstruktur für Tension Leg Plattform . . . . . . . . . . . . . . . . 
6.36 Mechanisches Modell für Tension Leg Plattform . . . . . . . . . . . . . 81

6.37 Vergleich der Geometrien und Lebensdauern für die optimierten Strukturen: TLP . . . . . . . . . . . . . . . . . . 85

6.38 Vergleich der Übertragungsfunktionen der dynamischen Spannungen der Tether . . . . . . . . . . . . . . . 86

6.39 Vergleich der Verteilungen der Schädigungen für das Haltenbanken-Gebiet 86

A.1 Quadratischer B-Spline . . . . . . . . . . . . . . . 101

B.1 Koordinatensysteme und Beschreibung eines Strukturelements . . . . . 103

B.2 Bewegungsfreiheitsgrade . . . . . . . . . . . . . . . . . . . . . . 104

B.3 Wellenpartikelbeschleunigung am kreiszylindrischen Strukturelement mit veränderlichen Querschnittsgrößen . . . . . . . . . . . . . . . 106

B.4 Berechnungsmodell zur Ermittlung der Druckkraft auf die in Längsrichtung eines Strukturelementes projizierte Fläche . . . . . . . . . . . 115

B.5 Beiwerte der hydrodynamischen Masse für harmonisch in Axialrichtung schwingende Körper (Clauss et al., 1992) . . . . . . . . . . . . . . . 116

B.6 Näherungsansatz für die hydrodynamische Masse mit zwei benachbarten Scheibenelementen . . . . . . . . . . . . . . . . . 118

B.7 Verdrängungszunahme bei kleinen Bewegungen . . . . . . . . . . . . 121

B.8 Krängungsmoment durch Schwerpunktsverschiebung . . . . . . . . . . . 123

B.9 Schematischer Ablauf des Programmes TFC . . . . . . . . . . . . . . . 128

B.10 Vergleich der gerechneten und experimentellen Übertragungsfunktionen bei einem Welleneinfallswinkel von $\varphi=0^{\circ}$. . . . . . . . . . . . 129

B.11 Vergleich der gerechneten und experimentellen Übertragungsfunktionen bei einem Welleneinfallswinkel von $\varphi=45^{\circ}$. . . . . . . . . . . 130

B.12 Vergleich der gerechneten und experimentellen Übertragungsfunktionen bei einem Welleneinfallswinkel von $\varphi=90^{\circ}$. . . . . . . . . . . 131

B.13 Vergleich der gerechneten und experimentellen Übertragungsfunktionen am Beispiel eines Caisson-Halbtauchers mit veränderlichen Querschnittsflächen: Welleneinfallswinkel $\varphi=0^{\circ}$. . . . . . . . . . 132

C.1 Schematische Darstellung des Diffraktion-Radiationsproblems . . . . . 135 


\section{Tabellenverzeichnis}

6.1 Definition der beiden Ausgangsstrukturen . . . . . . . . . . . . . . 45

6.2 Vergleich der gefundenen Minima nach den Optimierungsstrategien . . 47

6.3 Formparameter der gewählten Ausgangsstrukturen und optimierter Strukturen mit dem lokalen Optimierungsalgorithmus SQP . . . . . . . . . 55

6.4 Vergleich der Ergebnisse der globalen Optimierungsalgorithmen mit der lokalen SQP Strategie . . . . . . . . . . . . . . . 57

6.5 Ergebnisse mit den kombinierten Optimierungsstrategien . . . . . . . . 58

6.6 Vergleich der Optimierungsergebnisse bei Berücksichtigung des Zähigkeitseinflusses ........................ 67

6.7 Vergleich der Ergebnisse der hydrodynamischen Formoptimierungen für einen Halbtaucher mit $\triangle=50,000 m^{3} \ldots \ldots$. . . . . . . . . 75

6.8 Freie Variable der Ausgangsstruktur und der optimierten Strukturen . . 75

6.9 Verwendete Daten für die Ermüdungsanalyse . . . . . . . . . . . . . . 83

6.10 Vergleich der Ergebnisse der hydrodynamischen Formoptimierungen für eine Tension Leg Plattform . . . . . . . . . . . . . . . . . . . 83 


\section{Kapitel 1}

\section{Einleitung}

Mit dem weltweit zunehmenden Energiebedarf in den letzten sechs Jahrzehnten entwickelte sich die Offshore-Technik rapide. Eine der Hauptaufgaben der Offshore-Technik ist die Konzeption und Konstruktion von geeigneten Trägersystemen, die für die Exploration und Gewinnung von Erdöl und Erdgas aus Meereslagerstätten eingesetzt werden.

Meerestechnische Konstruktionen müssen für extrem raue Umweltbedingungen und große Wassertiefen entwickelt werden. Alle Offshore-Arbeiten, vor allem die Installation von Plattformen, Kranarbeiten oder das Setzen eines Blow-Out-Preventers gestatten nur geringe Bewegungen der Trägerstrukturen. Besonders Vertikalbewegungen sind sehr kritisch und müssen durch entsprechende Vorrichtungen ausgeglichen werden. Da die Möglichkeiten hierfür aber eingeschränkt sind, ist bei schwimmenden Strukturen die Minimierung der Bewegungen im Seegang ein wichtiges Entwurfsziel. Das Vordringen in Tiefwasser mit immer unwirtlicheren Seegangsbedingungen erfordert die ständige Verbesserung der Trägersysteme und die Entwicklung neuer Konzepte für OffshoreKonstruktionen.

In früheren Arbeiten von Clauss und Birk (1995b) und Birk (1998) konnte gezeigt werden, dass sich Seegangseigenschaften meerestechnischer Konstruktionen (wie Erregerkräfte, Bewegungen u.a. bis hin zu Einsatzzeiten) durch hydrodynamische Formoptimierung signifikant verbessern lassen. Als Optimierungsstrategie wurde dabei die Tangentensuchmethode (TSM) nach Hilleary (1966) eingesetzt. Es handelt sich hierbei um ein deterministisches, direktes Suchverfahren (siehe Abschnitt 4.3.1). Die mit der Tangentensuchmethode erzielten Ergebnisse stellen nur in einem begrenzten Bereich des Parameterraumes ein lokales Optimum dar (Birk, 1998). Diese intelligente Suchstrategie kann mit einem Sprungschritt aus der Ecke ${ }^{1}$ herauskommen, wobei zwischenzeitlich auch Punkte mit schlechtem Zielfunktionswert akzeptiert werden. Diese Eigenschaft unterscheidet TSM von anderen klassischen Suchalgorithmen (z.B. Gradientenverfahren). Bei praktischen Anwendungen ist dies jedoch schwer zu realisieren (Birk, 2003).

Ein Parameterraum kann mehrere Lösungen besitzen, deren Zielfunktionswerte klei-

\footnotetext{
${ }^{1} \mathrm{Im} n$-dimensionalen Fall stellt die Nebenbedingung eine Hyperebene dar. Durch die Schnittmenge der verschiedenen Hyperebenen kann eine enge "Ecke" des Variablenraumes vorkommen.
} 
ner als Nachbarpunkte sind. Diese Lösungen müssen nicht notwendigerweise die beste Lösung des Problems repräsentieren, aber sie sind die beste, erreichbare Lösung in einer bestimmten Subregion des Lösungsraumes. Solche Lösungen nennt man lokale Minima. Lokale Minima stellen in jedem Fall eine Verbesserung gegenüber dem Ausgangsentwurf dar und sind im Sinne des Anwenders ein Fortschritt. Zielfunktionen technischer Optimierungsaufgaben können multimodal sein, d.h. sie können mehrere Minima besitzen. Multimodal ist ein Problem, wenn entweder die Zielfunktion nicht überall konvex ist, oder mindestens eine Nebenbedingung konkav ist oder beides. Bei multimodalen Optimierungsaufgaben wächst die Gefahr, nur ein lokales Minimum zu finden. Selbst wenn die Optimierung mit unterschiedlichen Ausgangsstrukturen und Steuerparametern öfters wiederholt wird, kann das beste lokale, das sogenannte globale Minimum verfehlt werden. Obwohl der Einsatz der Tangentensuchmethode in die hydrodynamische Formoptimierung deutliche Verbesserungen des Seegangsverhaltens erzielen konnte, könnten noch wesentlich bessere Entwürfe möglich sein. Natürlich können die Suchstrategien, die ursprünglich für Funktionen mit einem Optimum (unimodale Zielfunktion) entwickelt wurden, für multimodale Fälle mit mehrfachen Anfangsvektoren verwendet werden. Es wird vermutet, dass bessere Entwürfe durch den Einsatz globaler Optimierungsstrategien erzielt werden können. Vor allem bei der Entwicklung neuartiger Strukturen, bei der nicht auf die Erfahrung aus früheren Entwürfen zurückgegriffen werden kann, und bei Fragestellungen mit vielen Optimierungsvariablen und Nebenbedingungen sollten globale Optimierungsalgorithmen mit größerer Wahrscheinlichkeit bessere lokale Minima bzw. das beste Minimum erzielen.

Ziel dieser Arbeit ist deshalb die Anwendung globaler Optimierungsstrategien auf meerestechnische Konstruktionen mit beliebigen Geometrien. Unterschiedliche Klassen von globalen Optimierungsalgorithmen werden in das bestehende Formoptimierungssystem integriert. Am Beispiel der hydrodynamischen Formoptimierung meerestechnischer Konstruktionen soll die Machbarkeit globaler Optimierung in einer komplexen technischen Anwendung aufgezeigt werden, wobei die Algorithmen hinsichtlich des Rechenaufwandes und der Güte des gefundenen Optimums untereinander sowie mit einer lokalen Strategie verglichen werden. Um einen gerechten Vergleich zu gewährleisten, sollen die Ergebnisse der einzelnen Fallstudien vorsichtig interpretiert werden.

In der vorliegenden Arbeit werden komplexe Formoptimierungsaufgaben mittels globaler Optimierungsstrategien gelöst, um bestmögliche Kombinationen der Entwurfsparameter für Offshore-Strukturen beliebiger Geometrien zu bestimmen. Die Erweiterung auf beliebige Geometrien bedeutet eine erhebliche Steigerung der Komplexität der Optimierungsaufgabe, da deutlich mehr Wahlmöglichkeiten für die freien Variablen zur Verfügung stehen. Zur Vergrößerung der Formenvielfalt bei der hydrodynamischen Formoptimierung wird ein erweitertes Formgenerierungsverfahren eingesetzt, das auf der Basis der Freiformflächen entwickelt wurde (Huang, 2000). Im Gegensatz zur Arbeit von Birk (1998) kann die Art der Querschnittsfläche für eine Strukturkomponente von Kreisen über Ellipsen, bis hin beliebigen Vierecken mit scharfen oder gerundeten Kanten variiert werden, um eventuell bessere Lösungen zu erhalten. Systematische Tests werden mit wachsender Anzahl der freien Variablen durchgeführt, um die Leistungsfähigkeit globaler Algorithmen in der Anwendung auf komplexe, technische Systeme zu 
untersuchen. In der Meerestechnik treten Optimierungsaufgaben mit vielen freien Variablen sehr häufig auf, da ständig neue Systemkonzepte entwickelt werden müssen, um den wachsenden Anforderungen an Sicherheit, Performance und Umweltverträglichkeit als auch den schwieriger werdenden Einsatzbedingungen (steigende Wassertiefe, Polarregion) gerecht $\mathrm{zu}$ werden.

Neben der Optimierung hydrodynamisch kompakter Konstruktionen werden auch hydrodynamisch transparente Strukturen als Anwendungsbeispiel betrachtet. Bislang wurde bei der Optimierung angenommen, dass die Veränderung der Übertragungsfunktion im Bereich der Resonanz- und Auslöschungsfrequenz keine Auswirkungen auf den Ablauf der Optimierung hat, da bei allen Entwürfen gleichermaßen Zähigkeitseinflüsse vernachlässigt wurden (Birk, 1998). Streng genommen gilt dies nur für eindeutig hydrodynamisch kompakte Strukturen. Kleinere Halbtaucher mit ca. 30,000 $\mathrm{m}^{3}$ Verdrängung gehören zum Übergangsbereich zwischen hydrodynamisch transparent und kompakt. Mit dem entwickelten TFC (Transfer Function Calculation) Programm kann der Einfluss der Zähigkeit auf das Ergebnis lokaler und globaler Optimierungsstrategien untersucht werden. Das TFC Programm wurde mit Versuchsdaten erfolgreich validiert (siehe Anhang B.8).

Zur Bewertung der Seegangseigenschaften einer Struktur in Bezug auf ihre gesamte Einsatzdauer an einer Lokation wurde bislang die statistische Verteilung der Seegangszustände in Form von diskreten Tabellen berücksichtigt (Birk, 1998). Dies hat den Nachteil, dass die Zielfunktion unempfindlich gegen kleine Geometrieänderungen wird: Weil der Grenzwert für die zulässige signifikante Wellenhöhe auf die Klasseneinteilung der Tabelle gerundet wird, erhält man für zwei unterschiedliche Optimierungsvariablen den gleichen Zielfunktionswert. Um kleine Verbesserungstrends der Zielfunktion nicht zu verlieren, wird in dieser Arbeit die diskrete Tabelle der statistischen Verteilung der Seegangszustände mit Hilfe einer numerischen Interpolation durch eine analytische Verteilungsfunktion ersetzt (Mathisen und Bitner-Gregersen, 1990).

Neben dem Stand der Technik findet sich im Kapitel 3 eine ausführliche Beschreibung der hydrodynamischen Formoptimierung im Entwurfsprozess. Anschließend werden im Kapitel 4 die in der hydrodynamischen Formoptimierung meerestechnischer Konstruktionen eingesetzten Optimierungsstrategien vorgestellt. Dabei werden nur die für einen Vergleich notwendigen, wesentlichen Eigenschaften erläutert. In Kapitel 5 Berechnung der Zielfunktionen werden kurz die drei in der automatisierten Optimierung zusammengefassten Schritte Geometriemodellierung, hydrodynamische Analyse und hydrodynamische Bewertung vorgestellt. Details über die Grundlagen hydrodynamischer Analyse finden sich im Anhang B und Anhang C. Obwohl sich die präsentierten Anwendungen auf schwimmende Strukturen und Minimierung der seegangsbedingten Bewegungen konzentrieren, sind andere Anwendungen ebenfalls realisierbar. Über die Arbeiten zur hydrodynamsichen Formoptimierung feststehender meerestechnischer Strukturen und Mehrkörpersysteme wird z.B. bei Clauss und Birk(1995a), Birk(1998) und Birk und Clauss(2001a) berichtet. Ergebnisse der hydrodynamischen Formoptimierung mit lokalen und globalen Optimierungsstrategien werden im Kapitel 6 präsentiert. Mit einem Schluss in Kapitel 7 und einer alphabetisch geordneten Literaturliste wird die Arbeit abgeschlossen. 


\section{Kapitel 2}

\section{Stand der Technik}

Der Einsatz meerestechnischer Konstruktionen in immer unwirtlicheren Seegebieten und die Entwicklung von Strukturen zur Erfüllung neuer Aufgaben erfordert eine optimale Anpassung der Systeme an Einsatzbedingungen und Zweck. Wichtig für die Sicherheit und Verfügbarkeit der teilweise gigantischen Offshore-Strukturen sind optimale Seegangseigenschaften. Im Entwurf wird daher die Geometrie variiert, um Kräfte und Bewegungen zu minimieren. Neben der Entwicklung und Verbesserung von CFDund CAD-Werkzeugen muss in der Forschung verstärkt die Integration der Werkzeuge in Entwurfsprozesse vorangetrieben werden (Johnson, 1990). Besonders erfolgversprechend ist die Kombination von Methoden aus CFD/CAD und der Nichtlinearen Programmierung (Optimierungsalgorithmen), wie dies im Bereich der Konstruktionsberechnung mit FE-Methoden bereits allgemeine Praxis ist (Catley et al., 1990).

Die meisten Anwendungen von numerischer, hydrodynamischer Analyse sowie Algorithmen der Nichtlinearen Programmierung untersuchen lediglich Spezialfälle, deren Methoden nur schwer auf andere Aufgaben übertragbar sind. Vasconcellos und Vassalos (1991) wenden die Direkte Suche auf die Optimierung der Hauptabmessungen eines schwimmenden Produktionssystems (FPS) an. Als Zielfunktion wird die Resonanzperiode der Tauchbewegung maximiert. Strukturgewicht, Deckslast und Stabilität werden ebenfalls einbezogen. Lokale Formänderungen wurden nicht berücksichtigt.

Zur Optimierung einer Anordnung von vertikalen schwimmenden Zylindern in Wellen setzt Kagemoto (1992) eine Straffunktionsmethode nach Fiacco und McCormick (1968) ein. Als Gütefunktion wurden entweder die gesamte Horizontalkraft, die gesamte Vertikalkraft oder die mittlere Driftkraft jeweils für eine spezielle Wellenlänge minimiert. Die Beschreibung des Srömungspotentials erfolgt analytisch nach Kagemoto und Yue (1986). Optimierte Anordnungen verschiedener Anzahlen vertikaler Zylinder und ausgesuchter Wellenlängen werden präsentiert. Die Methode ist jedoch nur für vertikale Zylinder zulässig und erlaubt keine Variation der Zylinderform selbst.

Die oben genannten Arbeiten verwenden keine praxisnahe Beschreibung des Seeganges. Die Ergebnisse sind daher nur eingeschränkt auf das Verhalten in realen Seegangszuständen übertragbar. Akagi und Ito (1984) greifen in ihrer Arbeit die spektrale Betrachtungsweise von Chou (1977) auf und minimieren die Varianz bzw. die Energie der Tauchbewegung eines Halbtauchers durch Variation der Hauptabmessungen. Der Seegangszustand wird dabei durch ein Energiedichtespektrum beschrieben, wo- 
mit die Anregung der Struktur über einen begrenzten Zeitraum genau genug erfasst wird. Energiedichtespektren wurden zuvor bereits von Söding und Häusler (1976) bei der Optimierung von SWATH-ähnlichen Körpern angewandt. In der Schiffsdynamik findet die Anwendung der Spektralanalyse bereits seit der wichtigen Publikation von St. Denis und Pierson (1953) statt.

Clauss und Birk (1995b) stellten ein komplexes Optimierungssystem vor, das die Optimierung von schwimmenden und bodengegründeten Systemen bis hin zu Mehrkörpersystemen erlaubt. Ausgehend von einem vorgegebenen Entwurf werden so lange Variationen der Geometrie durchgeführt, bis eine Form mit minimaler seegangsbedingter Ausfallzeit gefunden ist. Details über verschiedene Anwendungsbeispiele für hydrodynamische Formoptimierungen finden sich bei Clauss und Birk (1994b, 1995a, 1996, 1998a), Birk (1998) und Birk und Clauss (1999). Zur Ablaufsteuerung sind zwei deterministische Optimierungsalgorithmen im Programm implementiert (Hilleary,1966; Himmelblau und Paviani,1969). Beide Algorithmen konvergieren schnell zu einem lokalen Minimum wie am Beispiel einer schwerkraftgegründeten Plattform mit dem deutlichen Abfall des Zielfunktionswertes über die ersten 60 Iterationsschritte zu sehen ist (Birk, 1998).

Als wichtigen Preprozessor enthält das Optimierungssystem ein Formgenerierungsund Diskretisierungsmodul (Clauss und Birk, 1994a), das meerestechnische Konstruktionen durch affine Verzerrung einfacher Grundgeometrien (Kreis, Rechtecke) entlang einer Gerade erzeugt. Die einfache Geometrieverarbeitung beschränkt allerdings die Möglichkeiten des Optimierungssystems. Zur Vergrößerung der Formenvielfalt bei hydrodynamischer Formoptimierung wurde ein erweitertes Formgenerierungsverfahren entwickelt (Huang, 2000), wobei aus Grundkurven die Oberfläche der Strukturkomponenten durch modifizierte Sweeping-Operation erzeugt und als Non-Uniform-RationalB-Spline (NURBS) dargestellt wird. Mit Hilfe des erweiterten Formgenerierungsverfahren wurde das Optimierungssystem weiter entwickelt und neue Anwendungsbeispiele für hydrodynamische Formoptimierungen präsentiert (Birk und Clauss; 2001a, 2001b).

Die vorgestellten Arbeiten verwenden zur Lösung der Optimierungsaufgabe klassische Algorithmen der Nichtlinearen Programmierung für unimodale Funktionen. Die Zielfunktionen technischer Optimierungsaufgaben können multimodal sein. Aufgrund dieser multimodalen Eigenschaften des Lösungsraumes repräsentieren die Lösungen lokale Optima. Unterschiedliche Ausgangsstrukturen, aber auch Änderungen der Steuerparameter führen zu anderen optimierten Strukturen. Es kann deshalb das beste lokale, das sogenannte globale Optimum verfehlt werden. Die Kenntnis des globalen Optimums im Parameterraum würde eine deutliche Verbesserung des Entwurfs von Offshore-Strukturen bedeuten. Als Lösung bietet sich der Einsatz globaler Optimierungsalgorithmen an. Diese vergleichsweise neuen Algorithmen werden seit etwa 20 Jahren intensiv untersucht. Sie finden nur langsam Eingang in technische Fragestellungen, da sie normalerweise mehr Auswertungen der Zielfunktion als lokale Optimierungsalgorithmen benötigen. Der erhöhte Rechenaufwand muss gegenüber den zu erwartenden besseren Ergebnissen abgewogen werden.

Man unterscheidet drei wichtige Klassen von globalen Optimierungsalgorithmen: 
- Simulated Annealing (SA)

- Genetische Algorithmen (GA) bzw. Evolutionsstrategien (ES) und

- Branch-and-Bound Verfahren (BaB).

Daneben existieren noch eine ganze Reihe meist heuristischer, auf spezielle Probleme abgestellter Ansätze. Da diese keine große Verbreitung haben und kaum theoretische Grundlagen dazu erarbeitet worden sind, sind sie für dieses Projekt nicht in Betracht gezogen worden.

Die ersten beiden der oben genannten Klassen (SA,GA bzw. ES) sind stochastische Methoden, die auf der Nachahmung natürlicher Prozesse basieren. Simulated Annealing ${ }^{1}$ entstand aus einer Erweiterung des Metropolis-Algorithmus zur Lösung von Linienintegralen (Metropolis et al., 1953) um einen als Temperatur bezeichneten Relaxationsparameter (Kirkpatrick et al., 1983). Die Funktionsweise dieses Algorithmus wird an dem folgenden Beispiel verdeutlicht: Um Kristalle mit möglichst geringen Defekten (d.h. stabiler Zustand minimaler Energie) zu erzeugen, wird eine Schmelze hoch erhitzt. Kontrolliertes Absenken der Temperatur ermöglicht den Atomen, die optimale Anordnung einzunehmen. Bei hohen Temperaturen sind noch große Positionsänderungen möglich, die beim Abkühlen immer kleiner werden, bis die Kristallstruktur erstarrt ist. Zur Lösung von Optimierungsaufgaben wird diese Grundidee des Annealing simuliert. Dabei stellt der Kontroll-Parameter T, der zur Steuerung der Wahrscheinlichkeiten für die Änderungen der freien Variablen des Systems eingesetzt wird, das Analogon zur Temperatur $T$ bei einem Werkstück dar: Bei hoher Temperatur haben große Änderungen auch große Wahrscheinlichkeiten. Bei niedrigen Temperaturen treten meist nur noch kleine Änderungen auf. Eine geringe Wahrscheinlichkeit für große Sprünge im Parameterraum ist allerdings immer noch vorhanden. Wird für einen neuen Parametervektor ein schlechterer (größerer) Zielfunktionswert gefunden, wird anhand einer Akzeptanzfunktion entschieden, ob der schlechtere Wert angenommen oder verworfen wird. Parametervektoren mit besseren Zielfunktionswerten werden immer als neue Ausgangswerte akzeptiert. Die Akzeptanzwahrscheinlichkeit schlechterer Zielfunktionswerte sinkt mit der Temperatur T. Durch die Möglichkeit, auch schlechtere Funktionswerte beizubehalten, wird die Gefahr reduziert, in lokalen Minima stecken zu bleiben.

Simulated Annealing stellt geringe Ansprüche an die Gütefunktion. Selbst unstetige und nicht differenzierbare Funktionen sind zulässig (Rosen, 1992). Da der ursprüngliche SA-Algorithmus nur sehr langsam konvergiert, wurden von verschiedenen Autoren Varianten mit rascherer Temperaturabsenkung und besserer Konvergenz entwickelt (Ingber, 1989). Bennage und Dhingra (1995) verwenden einen SA-Algorithmus um Gewicht und Verformung eines räumlichen Tragwerkes zu minimieren. In der vorliegenden Arbeit wird der Adaptive Simulated Annealing (ASA) Algorithmus von Ingber $(1993,1996)$ verwendet, der schon in mehreren Wissenschaftsbereichen erfolgreich eingesetzt wird (Sen und Stoffa, 1995; Mayer et al., 1996). Dieser Algorithmus erlaubt die Berücksichtigung von Nebenbedingungen in Form von Straffunktionen sowie die Parallelisierung des Algorithmus (Ingber, 1992).

\footnotetext{
${ }^{1}$ annealing, engl. für das Normalisieren / Glühen von Stahl
} 
Genetische Algorithmen (GA) und Evolutionsstrategien (ES) entstanden Anfang der siebziger Jahre, wobei die Entwicklung der GA auf Arbeiten von Holland (1975) in den USA zurückgeht, während die Entwicklung von ES durch Schwefel (1971) und Rechenberg (1973) in Deutschland initiiert wurde. Beide Algorithmen werden kontinuierlich weiterentwickelt (Holland, 1992; Rechenberg, 1994). Die Basis beider Verfahren besteht aus der Adaption von Methoden der biologischen Evolution für Optimierungsprozesse. Obwohl der theoretische Nachweis für das sichere Auffinden eines globalen Optimums bislang nicht erbracht ist, existieren eine Vielzahl erfolgreicher technischer Anwendungen (Arciszewski et al., 1999; Deb, 1999; Cvetkovic und Parmee, 1999; Kitamura, 2001; Marcelin, 1999; Murawski et al., 2000). Hierbei beziehen sich die Optimierungsaufgaben meistens auf den Konstruktionsentwurf. Kitamura (2001) verwendet z.B. einen Genetischen Algorithmus um die Konstruktionskosten eines Schiffsmaschinenraumes zu minimieren. Eine gute Übersicht über Anwendungen von GA bieten Schwefel et al. (1993) und Bentley und Wakefield (1996). Zur effizienten Optimierung werden GA mit deterministischen Suchstrategien gekoppelt, da die lokalen Algorithmen schneller konvergieren. Lee et al. (2002) zeigen eine effektive Anwendung der globalen und lokalen (hybride) Optimierungsmethode, bei der ein direktes Suchverfahren gezielt mit GA kombiniert wird.

Obwohl die Grenzen zwischen Genetischen Algorithmen und Evolutionsstrategien immer mehr verwischen, unterscheiden sich GA und ES in der Umsetzung und der Gewichtung der drei wichtigsten Evolutionsmechanismen Selektion, Rekombination und Mutation. Genetische Algorithmen erzielen Fortschritte durch ausgefeilte Rekombinationsstrategien, während bei ES Mutationen und die Steuerung der Schrittweiten eine größere Rolle spielen (Schoeneburg et al., 1994). Während bei Evolutionsstrategien die freien Variablen als reellwertige Vektoren abgespeichert werden, werden bei GA die freien Variablen üblicherweise in binärer Form kodiert. Dies erweist sich allerdings als ungünstig bei der Speicherung reeller Zahlenwerte, da die einzelnen Gene im Code nicht gleich gewichtet sind. Ein Bit hat eine um so größere Wichtung, je weiter links es steht. Demzufolge ist die Topologie des Raumes der sog. Phänotypen anders als die Topologie des Kodierungsraumes, da Nachbarschaftsbeziehungen verändert werden. Diese Schwierigkeit tritt bei der reellwertigen Kodierung eines Individuums nicht auf. Wie Michalewicz (1994) in seiner Untersuchung durch den Vergleich der binären Kodierung von GA mit der reellwertigen Kodierung zeigt, ist die reellwertige Repräsentation hinsichtlich der Genauigkeit und des Rechenaufwandes gegenüber der binären Kodierung deutlich effizienter. In der hydrodynamischen Formoptimierung wird deshalb ein Genetischer Algorithmus von Houck et al. (1995b) eingesetzt, bei dem die freien Variablen direkt als reellwertige Vektoren eingegeben werden können. Dieser in Matlab entwickelte Algorithmus wurde mit einer Reihe von nichtlinearen und multimodalen Testproblemen getestet (Houck et al., 1995a). Aufgrund der einfachen Übertragbarkeit und der zusätzlich eingebauten, numerischen Funktionen von Matlab lässt sich dieser Genetische Algorithmus einfach in das hydrodynamische Formoptimierungssystem integrieren.

Branch-and-Bound (BaB) Verfahren gehören zur Gruppe der deterministischen Verfahren (Horst und Tuy, 1993). Während die stochastischen Verfahren Vorgänge der 
Natur nachbilden, sind BaB-Verfahren mathematisch orientiert. Schon früh entstanden theoretische Arbeiten zu Eigenschaften und Konvergenzkriterien (Mitten, 1970). Die Grundidee von BaB-Verfahren ist, dass man nicht wirklich alle zulässigen Lösungen untersucht, sondern große Untermengen von zulässigen Lösungen herausfiltert, die das globale Optimum nicht enthalten können (Weihe, 1998). Dazu wird der Parameterraum in kleinere Abschnitte (Branching) aufgeteilt, die zur weiteren Bearbeitung in einer Liste abgespeichert werden (siehe Abschnitt 4.4.3). Für jeden Abschnitt wird eine untere und obere Grenze der Zielfunktionswerte bestimmt (Bounding). Jede zulässige Lösung ergibt eine obere Schranke für den optimalen Zielfunktionswert und wird in der Regel über eine Näherungslösung bestimmt und im Laufe des Verfahrens aktualisiert (Grossmann und Terno, 1997). Durch den Vergleich der unteren und oberen Schranken unterschiedlicher Abschnitte, können alle Abschnitte, deren untere Grenze größer ist als die obere Schranke anderer, von der weiteren Suche ausgeschlossen werden (Cut-Off-Test). Der abzusuchende Parameterraum wird dadurch schnell und deutlich verkleinert. Abschnitte, die nicht durch den Cut-Off-Test von der Bearbeitungsliste entfernt wurden, werden erneut aufgeteilt und die entstehenden Abschnitte werden in die Bearbeitungsliste eingefügt. Bei Optimierungen mit kontinuierlichen Variablen wird der Prozess beendet, wenn das Intervall mit dem besten (globalen) Minimum eine vorgegebene Größe unterschreitet, bzw. die obere Schranke für das globale Optimum mit der unteren Schranke eines Abschnittes zusammenfällt. Gute Approximation der unteren und der oberen Schranken sind entscheidend für den Erfolg des BaB-Verfahren. Sie bestimmen die Größe des Branch-and-Bound Baumes und damit letztlich das Laufzeitverhalten des Algorithmus. Ferner sind sie wichtig zur Beurteilung der Güte der ermittelten Lösungen.

BaB-Algorithmen erbringen unter gewissen Voraussetzungen nicht nur eine obere und untere Grenze des Funktionswertes für das globale Optimum (Einschließung) sondern auch einen Nachweis über dessen Eindeutigkeit. Besonders effektiv können BaB-Verfahren eingesetzt werden, wenn detaillierte Kenntnisse über die Beschaffenheit der Zielfunktion verfügbar sind. Im Vergleich zu den stochastischen Methoden sind BaB-Verfahren schwierig zu implementieren. Insbesondere die Berechnung der unteren Grenze der Zielfunktion für einen Parameterabschnitt stellt eine hohe Hürde dar. Ein Schwerpunkt der aktuellen Forschung auf diesem Gebiet bildet der Einsatz von Intervallarithmetik (siehe z.B. Ratschek und Rokne, 1988; Hansen, 1992; Ratz, 1995; Kearfott, 1997). In der hydrodynamischen Formoptimierung erscheint der Einsatz von Intervallarithmetik derzeit schwierig, da das äußerst komplizierte mathematische Modell der Zielfunktion keinen expliziten Zusammenhang zwischen freien Variablen und Gütefunktion enthält. Praktikabel für die Berechnung der unteren Zielfunktionsgrenze erscheinen hier Ansätze, bei denen die Gütefunktion in einem kleinen Abschnitt durch konvexe Funktionen nach unten abgeschätzt wird (siehe z.B. Horst und Tuy, 1993; Epperly, 1995; Floudas et al., 1996; Royo und Sahinidis, 1996). Das Minimum des Abschnittes kann dann mit lokalen Algorithmen bestimmt werden, da konvexe Funktionen unimodal sind. 


\section{Kapitel 3}

\section{Hydrodynamische Formoptimierung}

Ziel eines jeden Entwurfsprozesses ist die schnelle Entwicklung einer zulässigen Lösung. Als Bewertungskriterien dienen Maßstäbe, die die Machbarkeit, Sicherheit, Wirtschaftlichkeit und Einsetzbarkeit des Entwurfs beurteilen, jedoch sind diese Kriterien nicht immer formal analytisch ausformuliert. Natürlich muss der Entwurfsprozess unter Beachtung der zur Verfügung stehenden Ressourcen an Zeit, Arbeitskraft und finanziellen Mitteln ablaufen. Wie von Evans (1959) eingeführt, werden Entwurfsprozesse häufig als Spirale dargestellt (Abb.3.1). Die Speichen des Spiralrades stellen die unterschiedlichen Teilaspekte der Entwurfsaufgabe dar, die iterativ wiederholt durchlaufen werden. Die Kontrolle, wie oft die Schritte durchgeführt werden und wann das Ergebnis als optimal betrachtet wird, liegt in der Hand des Entwerfers. Der in allen Bereichen der Analyse heute nicht mehr wegzudenkende Computer bietet jedoch auch Möglichkeiten, den Entwurfsprozess zumindest in Teilabschnitten zu automatisieren und für optimale Ergebnisse zu sorgen.

Aus den vielen Teilaufgaben des Entwurfsprozesses meerestechnischer Konstruktionen wird hier die Entwicklung der Rumpfform nach hydrodynamischen Kriterien als formale Optimierungsaufgabe betrachtet (siehe Abb.3.1). Die Kontrolle des (Teil-) Entwurfsprozesses wird von Optimierungsalgorithmen übernommen. Eine Untermenge der das System definierenden Größen, die freien Variablen, wird vom Optimierungsalgorithmus mit dem Ziel variiert, ein Entscheidungskriterium -die sogenannte Zielfunktionzu minimieren. Vorgegebene Nebenbedingungen wie z.B. hydrostatische Stabilität sind gleichzeitig zu erfüllen. Die Automatisierung des Entwurfsprozesses mit Optimierungsalgorithmen setzt voraus, dass Zielfunktion und Nebenbedingungen in mathematischen bzw. numerischen Modellen formuliert werden. Im Falle der hydrodynamischen Formoptimierung sind drei Schritte zur Berechnung der Zielfunktion notwendig (Abb. 3.1):

- Geometriemodellierung (CAD)

- Hydrodynamische Analyse (CFD) und

- Bewertung der Zielfunktion.

Alle dazu notwendigen Schritte und Schnittstellen müssen automatisiert werden. 


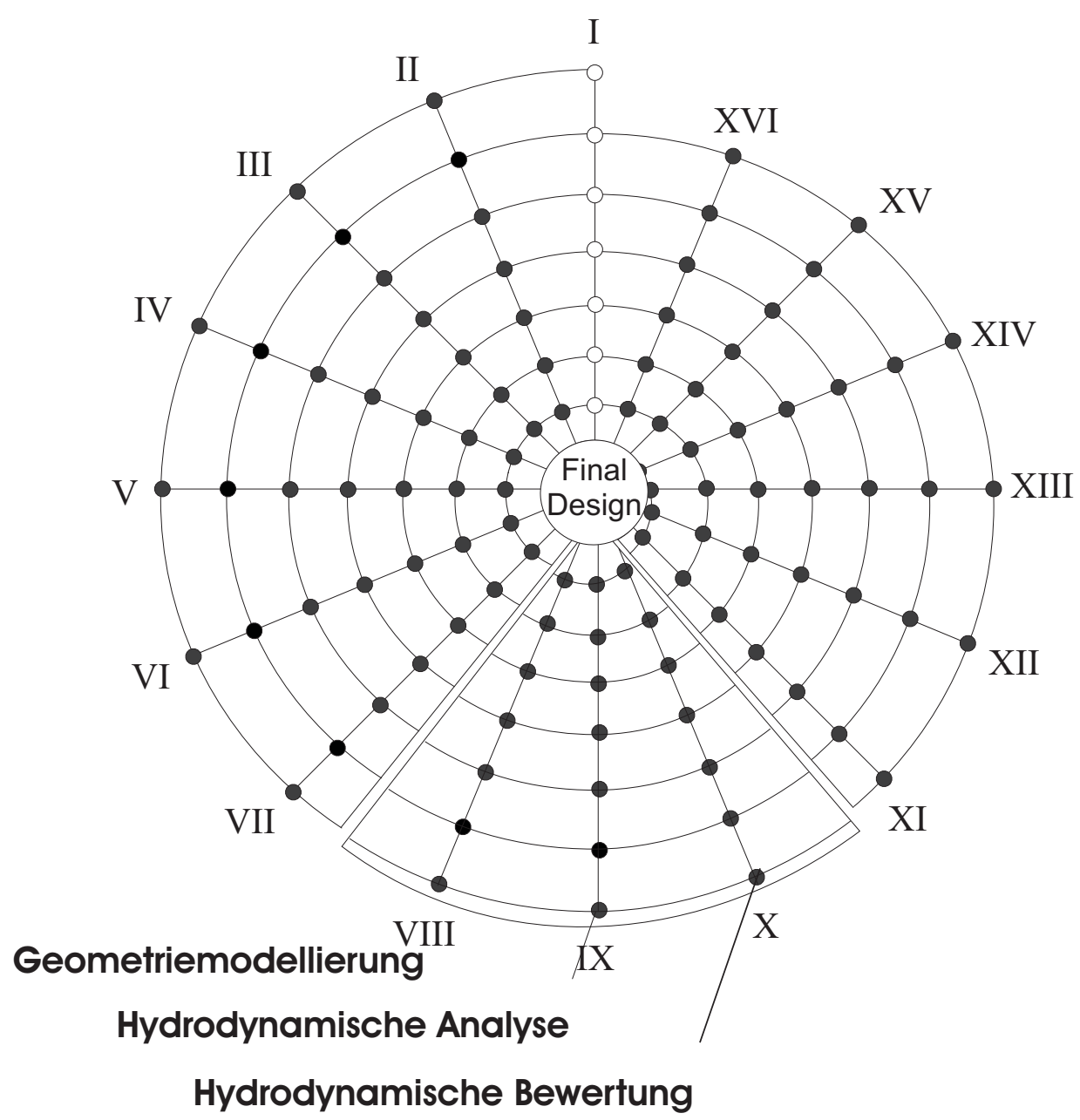

Abbildung 3.1: Teilaufgaben der hydrodynamischen Formoptimierung im Entwurfsprozess (Evans, 1959)

Die Einführung von Optimierungsalgorithmen in den Entwurfsprozess bedeutet eine erhebliche Umstellung für den Entwerfer. Abb.3.2 verdeutlicht die generellen Unterschiede. Im traditionellen Formentwurf wird mit interaktiven CAD Programmen die Rumpfgeometrie erzeugt. Aus Zeitersparnisgründen wird meist, vor allem in der Schiffstechnik, ein vorhandener Entwurf entsprechend modifiziert. Die Rumpfeigenschaften (Glattheit, Hydrostatik, etc.) werden mit Rechnerunterstützung vom Entwerfer kontrolliert, bevor die hydrodynamische Analyse gestartet wird. Anschließend wird vom Ingenieur anhand der Ergebnisse der hydrodynamischen Analyse eine Bewertung des Entwurfs vorgenommen. Das Erzeugen von Entwurfsvarianten und das wiederholte Durchführen der Analyse benötigt viel Zeit. Aufgrund der zeitlichen und wirtschaftlichen Restriktionen kann der Entwurfsprozess schon beendet werden, sobald der aktuelle Entwicklungsstand die Mindestvoraussetzungen erfüllt. Die optimale, d.h. beste Lösung ist somit schwer zu erreichen. Im Gegensatz dazu stellt die Verwendung von Optimierungsalgorithmen eine optimale Lösung des Teilproblems sicher. In der hydro- 
Traditioneller Rumpfentwurf

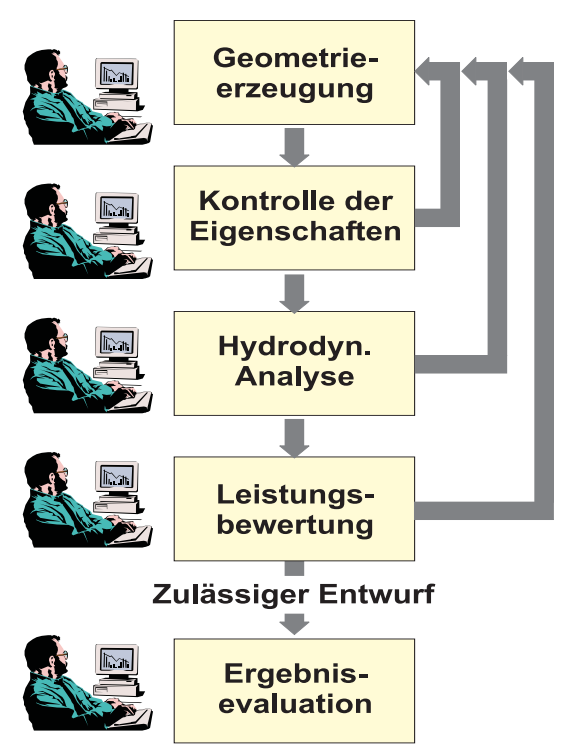

Hydrodynamische Formoptimierung

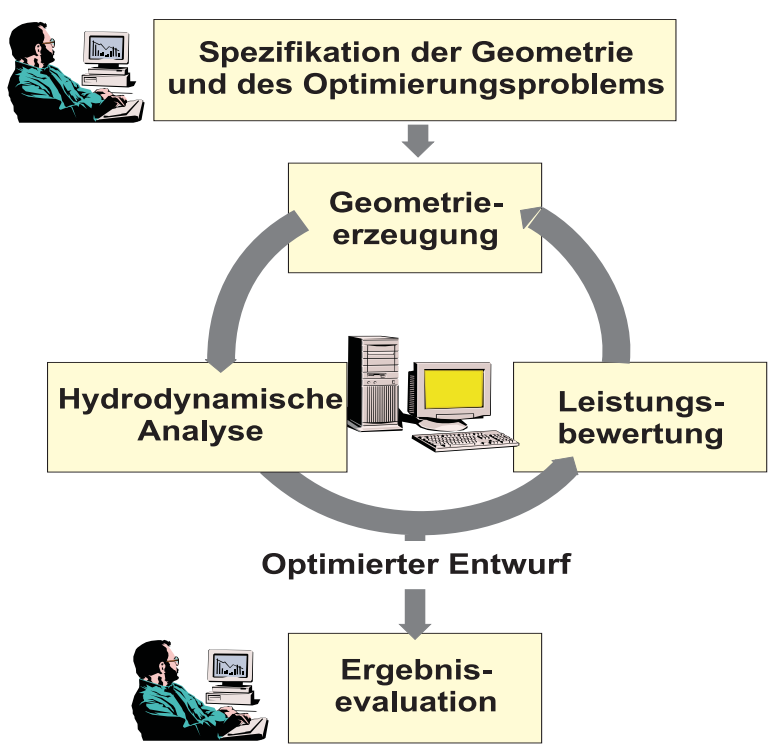

Abbildung 3.2: Traditioneller, interaktiver Rumpfentwurf versus hydrodynamische Formoptimierung (Birk et al., 2002)

dynamischen Formoptimierung wird der Ingenieur von den Routineaufgaben befreit, da der Rechner die Aufgabe des Erzeugens der Entwurfsvarianten und deren Analyse übernimmt. Der Arbeitsschwerpunkt des Ingenieurs verschiebt sich hin zu Definition und Vorbereitung der Optimierungsaufgabe und es bleibt mehr Zeit für die wichtige Kontrolle des Endergebnisses. Ist dieses nicht zufriedenstellend, wird die Optimierungsaufgabe verändert (z.B. die Randbedingungen) und nochmals gelöst.

Hydrodynamische Formoptimierung kann in der Praxis nur dann erfolgreich sein, wenn das System ausreichende Fähigkeiten zur parametrischen Geometrieerzeugung besitzt, da nur die vom System erzeugbaren Entwürfe die potentielle Lösungsmenge der Optimierungsaufgabe bilden. Auf dem Gebiet des parametergesteuerten Formentwurfs wurde in den letzten Jahren intensiv geforscht und Methoden und Softwaretools entwickelt (Birk und Harries, 2000, Abt et al., 2001). Die hydrodynamische Analyse ist wegen der langen Rechenzeiten meist bereits auf den automatisierten Betrieb ausgelegt, jedoch muss zusätzlich die Übergabe der Geometrie von der Modellierung zum CFDProgramm (Gittergenerierung) automatisiert werden. Dies ist heute für sogenannte Randelementverfahren (Panelmethoden) gut möglich, aber im Falle von Navier-StokesLösern noch nicht Stand der Technik.

Es ist zwar generell wünschenswert aber nicht unbedingt notwendig, dass die verwendeten Analyseprogramme korrekte Absolutwerte mit hoher Genauigkeit ermitteln können. In jedem Fall müssen sie die von Formänderungen verursachten Trends richtig wiedergeben. Mit den Ergebnissen der hydrodynamischen Analyse (Drücke, Kräfte, Bewegungen, etc.) muss eine Bewertung des Entwurfs vorgenommen werden. Da sich unter Umständen manche der Kriterien (z.B. Sicherheit gegen Wirtschaftlichkeit) entgegenwirken, ist eine Automatisierung und Zusammenfassung in einer Zielfunktion oft 
schwierig. Es existieren für Optimierungsprobleme mit konkurrierenden Zielfunktionen Lösungsstrategien, z.B. Multicriteria Optimization (siehe Osyczka (1984)).

Bei meerestechnischen Konstruktionen sind optimale Seegangseigenschaften von entscheidender Bedeutung für einen ökonomischen und sicheren Betrieb. Aussagekräftige Zielfunktionen können auf der Basis linearer Seegangs- und Strukturmodelle verknüpft mit der Seegangsstatistik erstellt werden (Clauss und Birk, 1998a; Birk und Clauss, 2001b).

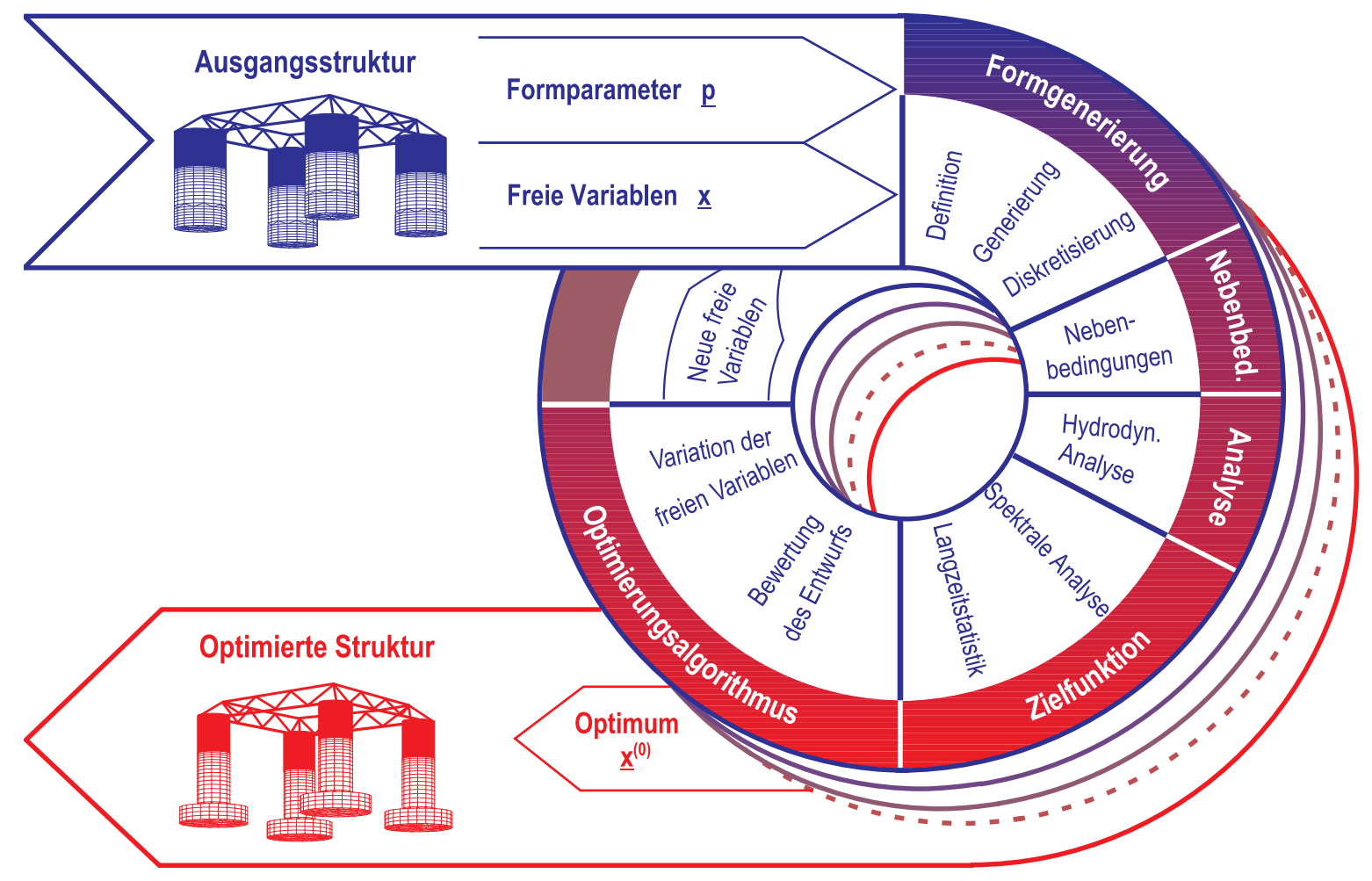

Abbildung 3.3: Ablauf der hydrodynamischen Formoptimierung (Birk, 1998)

Abb. 3.3 zeigt den Ablauf der hydrodynamischen Formoptimierung. Vor Beginn der Optimierung müssen die Zielfunktion, die Nebenbedingungen und aus den vorhandenen Formparametern die freien Variablen ausgewählt werden. Wie in Abb 5.1 beispielsweise aufgeführt, können alle Parameter der Struktur in beliebiger Kombination als freie Variable für die Optimierung gewählt werden. Meistens muss auch eine Ausgangsstruktur angegeben werden. Manche Algorithmen erzeugen diese durch zufällige Auswahl von Werten der selektierten freien Variablen selbst. Mit diesen Anfangswerten beginnt der Optimierungsalgorithmus die Schleife aus Geometrieerzeugung, hydrodynamischer Analyse und hydrodynamischer Bewertung. In Abhängigkeit von den Änderungen der Zielfunktion wird vom Optimierungsalgorithmus der Vektor der freien Variablen modifiziert, um in möglichst wenigen Schritten ein Minimum der Zielfunktion aufzufinden. Die Abbruchkriterien sind von Algorithmus zu Algorithmus unterschiedlich, bauen aber meist darauf, dass in einer Umgebung eines aktuellen Bestpunktes $\underline{x}^{*}$ oder innerhalb einer vorgegebenen Toleranz (z.B. Anzahl der Zielfunktionsauswertungen) keine bessere 
Lösung mehr ermittelt werden kann. 


\section{Kapitel 4}

\section{Optimierungsstrategien}

Um ein technisches Entwurfsproblem durch Optimierungsalgorithmen zu lösen, muss zunächst ein entsprechendes mathematisches Modell formuliert werden. Insbesondere sind folgende Teile des Modells zu definieren:

- die Zielfunktion bzw. das Gütekriterium (measure of merit), das die Qualität des Entwurfs ausdrückt,

- freie Variablen, welche die Eigenschaften des Entwurfs beschreiben und während des Optimierungsprozesses geändert werden, und

- Nebenbedingungen, die sicherstellen, dass der Entwurf technisch und ökonomisch zulässig ist.

Obwohl die Zielfunktion und die Nebenbedingungen von vielen Parametern abhängen können, sind manche Parameter nicht unter der Kontrolle des Entwerfers, d.h. sie sind meistens unveränderlich, sondern an den spezifischen Werten durch Anforderungen festgelegt. Nur die Teilmenge der Parameter, die als freie Variablen ausgewählt werden, kann während des Entwurfsprozesses geändert werden. Bei technischen Entwurfsproblemen spielen Nebenbedingungen eine wichtige Rolle im Optimierungsprozess. Man unterscheidet zwischen Gleichheits- und Ungleichheitsnebenbedingungen, die Kurven bzw. Hyperflächen (oder Hyperflächenschar von Isoparameterflächen) im $n$-dimensionalen Raum der freien Variablen bilden.

Das mathematische Modell muss einen Zusammenhang zwischen den gewählten freien Variablen $\underline{x}=\left\{x_{1}, x_{2}, \ldots, x_{n}\right\}$ sowie zusätzlichen, unveränderlichen Parametern $\underline{p}$ und der Zielfunktion $f(\underline{x}, \underline{p})$ herstellen. In den wenigsten Fällen wird dieses Modell nur aus einer expliziten Gleichung für die zu minimierende Zielfunktion bestehen.

\subsection{Mathematische Formulierung der Optimierungs- aufgabe}

Optimierungsprobleme mit Nebenbedingungen lassen sich allgemein als Minimierungsaufgaben formulieren: Für eine Zielfunktion $f(\underline{x})$ soll der Vektor der freien Variablen 
$\underline{x}^{*}$ gefunden werden, der ein Minimum der Funktion $f$ definiert und gleichzeitig die gegebenen Nebenbedingungen $h\left(\underline{x}^{*}\right)$ und $g\left(\underline{x}^{*}\right)$ erfüllt. Die Optimierungsaufgabe lautet also:

$$
\begin{aligned}
& \text { Finde den Vektor } \underline{x}^{*}=\left(x_{1}, x_{2}, \cdots, x_{n}\right)^{T^{*}}, \\
& \text { der die Gleichung } \\
& f\left(\underline{x}^{*}\right)=\min [f(\underline{x})]
\end{aligned}
$$

und die Nebenbedingungen

$$
\begin{gathered}
h_{j}\left(\underline{x}^{*}\right)=0 \quad j=1 \ldots m \\
g_{j}\left(\underline{x}^{*}\right) \geq 0 \quad \quad j=m+1 \ldots p \\
\underline{x}_{l} \leq \underline{x}^{*} \leq \underline{x}_{u} \\
\text { erfüllt. }
\end{gathered}
$$

Die Nebenbedingungen $h_{k}$ und $g_{j}$ sind somit jeweils als Gleichungen und Ungleichheiten formuliert. Ist z.B. der Wert der Ungleichheits-Nebenbedingungsfunktion negativ, wird die Nebenbedingung verletzt, d.h. der zugehörige Vektor der freien Variablen ist unzulässig. Speziell bei Sequential Quadratic Programming werden Nebenbedingungen und Zielfunktion zu generalisierter Lagrangefunktion zusammengefasst. Umfassende Darstellungen dieser Theorie finden sich z.B. bei Himmelblau (1972), Bertsekas (1999). Bei anderen Algorithmen werden Nebenbedingungen meist als Straffunktionsmethoden berücksichtigt.

\subsection{Klassifizierung der Optimierungsalgorithmen}

Im praktischen Sinne ist die Optimierung wahrscheinlich so alt wie die Menschheit. Der Wunsch, das Resultat von Entscheidungen bewusst zu optimieren, ist ein einzigartiges, menschliches Merkmal. Obwohl man den Optimierungsprozess der Natur nicht genau wissen kann, ist der Wunsch und die Fähigkeit des Menschen, Entscheidungen zu optimieren, vor allem die Auswahl von Alternativen unstrittig. Die Optimierung ist ein bewusstes oder unterbewusstes menschliches Verhalten. Eine Übersicht über die Frühgeschichte der Optimierung findet man bei Nowacki (2003).

Im Laufe der vergangenen vier Jahrzehnte wurde zur Lösung der Optimierungsaufgabe (4.1) eine Vielzahl numerischer Verfahren entwickelt (siehe z.B. Himmelblau, 1972; Fletcher, 1987). Die Aussage, welcher Algorithmus für welche Aufgabe besser geeignet ist, kann durch die Analyse der Funktionen auf statistischer Grundlage beantwortet werden. Tests mit unterschiedlichen Anwendungen sollen ein deutliches Bild liefern, mit welchen Algorithmen meerestechnische Konstruktionen mit den besten Seegangseigenschaften effektiv entwickelt werden können.

Je nachdem aus welcher Perspektive das Optimierungsproblem betrachtet wird, kann das Problem in unterschiedlicher Weise eingestuft werden. Bei Klassifizierung nach Art der Zielfunktion wird zwischen lokalen und globalen Algorithmen unterschieden. So genannte lokale Optimierungsstrategien sind für uni-modale Zielfunktionen entwickelt worden, d.h. ihre Konvergenznachweise sind nur für Zielfunktionen mit einem 
einzigen Minimum gültig. Sie können natürlich auch auf multi-modale Zielfunktionen angewandt werden, jedoch besteht die Gefahr, dass aufgrund der multimodalen Eigenschaften der Zielfunktion nur ein lokales Minimum gefunden wird. Die Chancen, das globale Minimum zu finden, erhöhen sich, wenn der Variablenraum von unterschiedlichen Startpunkten aus durchsucht wird. Allerdings vergrößert sich der Rechenaufwand entsprechend.

Globale Optimierungsstrategien sollen bei multi-modalen Zielfunktionen das beste aller lokalen Minima (globales Minimum) finden. Viele der Algorithmen ahmen in der Natur vorkommende Prozesse nach und sind beim Auffinden des globalen Minimums überraschend erfolgreich. Der Rechenaufwand ist im Vergleich zu lokalen Algorithmen meist erheblich größer. Es bleibt dem Anwender überlassen, zu entscheiden, ob eine lokal optimale Lösung ausreichend ist oder ob mit mehr Zeitaufwand und einem gewissen Unsicherheitsfaktor behaftet nach dem besten Entwurf gesucht werden muss.

Zur Lösung der Optimierungsaufgabe (4.1) wird gewöhnlich ein Anfangsvektor der freien Variablen $\underline{x}^{(1)}$ vom Anwender ausgewählt. Daraus wird eine Reihe von Vektoren der freien Variablen $\left\{\underline{x}^{(k)}\right\}$ erzeugt, die den Zielfunktionswert schrittweise verkleinert $f\left(\underline{x}^{(k+1)}\right)<f\left(\underline{x}^{(k)}\right)$. Für die Bestimmung der neuen Vektoren der freien Variablen gilt:

$$
\underline{x}^{(k+1)}=\underline{x}^{(k)}+\alpha^{(k)} \cdot \underline{d}^{(k)} .
$$

Der Parameter $\alpha^{(k)}$ bezeichnet die Schrittweite und $\underline{d}^{(k)}$ den Vektor der Suchrichtung. ${ }^{1}$ Man möchte nun einen Algorithmus haben, um ein Minimum möglichst in wenigen Schritten zu finden.

Bezüglich der Methodik, die zur Modifikation der freien Variablen von Optimierungsschritt zu Optimierungsschritt verwendet wird, unterteilt man die Algorithmen in deterministische und stochastische (probabilistische) Verfahren:

- Deterministische Verfahren ändern die freien Variablen nach algebraischen, festgelegten Regeln. Der Fortgang der Optimierung entscheidet, wann welche Regel zur Anwendung kommt. Die Änderungen der freien Variablen von $\underline{x}^{(k)} \mathrm{zu} \underline{x}^{(k+1)}$ sind daher vorhersehbar und beruhen auf den Informationen, die in den vorhergehenden Optimierungsschritten gewonnen werden.

- Bei stochastischen Verfahren wird eine Zufallskomponente bzw. eine Wahrscheinlichkeitsdichteverteilung in die Änderung der freien Variablen eingebaut. Deshalb sind die Bewegungen des Vektors der freien Variablen "unvorhersehbar".

Lokale Verfahren sind meist deterministisch, während die überwiegende Anzahl der globalen Algorithmen stochastischer Natur ist. Die deterministischen Methoden können wiederum in zwei Kategorien unterteilt werden. Wenn die Methode zur Bestimmung eines Optimums die ersten partiellen Ableitungen bzw. Krümmungsinformationen benötigt, wird diese Methode Gradientenverfahren genannt. Falls die Methode nur auf Zielfunktionswerten beruht und nicht versucht, die Ableitungen und Krümmungen auszuwerten oder zu approximieren, gehört sie zu den Suchmethoden.

\footnotetext{
${ }^{1}$ Der Parameter $\alpha^{(k)}$ kann auch ein Vektor sein, da die Schrittweiten in jede Richtung verschieden sein können.
} 


\subsection{Lokale Optimierungsstrategien}

\subsubsection{Tangentensuchmethode (TSM)}

Search methods oder Suchmethoden sind in den 1960-er und in frühen 1970-er Jahren entwickelt worden (Zoutendijk,1960; Hooke und Jeeves,1961) und werden noch häufig verwendet. Powell $(1970,1971)$ und Himmelblau (1972) geben einen umfassenden Überblick über die Entwicklungen dieser Optimierungsalgorithmen. Als Beispiel für eine intelligente Suchmethode wird hier die sogenannte Tangent Search Method oder Tangentensuchmethode (TSM) nach Hilleary (1966) kurz präsentiert, die eine klassische Suchstrategie für Optimierungsaufgaben mit Ungleichheitsnebenbedingungen ist.

Die Tangentensuchmethode vereinigt zwei unterschiedliche Algorithmen: Der Begriff Tangente bezieht sich hier auf die Methode der zulässigen Richtungen (Klingman und Himmelblau, 1964). Im zulässigen Bereich, d.h. wenn keine Nebenbedingungen verletzt ist, wird eine modifizierte direkte Suche nach Cooper und Glass (1965) durchgeführt. Um das zulässige Gebiet nicht zu verlassen, sucht der Algorithmus möglichst tangential zur Hyperfläche der Nebenbedingungen nach besseren Lösungen im Raum der freien Variablen, wann immer eine Nebenbedingung verletzt wird. Die notwendigen Ableitungen der Nebenbedingungen nach den freien Variablen werden durch einfache numerische Differenzenquotienten approximiert. TSM wurde in verschiedene hydrodynamische Optimierungsaufgaben mit bis zu 20 freien Variablen erfolgreich eingesetzt (siehe z.B. Papanikolaou et al., 1991; Clauss und Birk, 1994a; Birk, 1998; Birk und Clauss, 2001a).

In engen "Ecken" des Variablenraumes kann TSM mehrmals hintereinander eine Nebenbedingung verletzen. Es wird dann versucht mit einem Sprungschritt aus der Ecke herauszukommen, wobei zwischenzeitlich auch Punkte mit schlechtem Zielfunktionswert akzeptiert werden. Führt dies nicht zum Erfolg, wird die Suche am letzten Bestpunkt mit verkleinerter Schrittweite wieder fortgesetzt (Hilleary, 1966).

\subsubsection{Sequential Quadratic Programming (SQP)}

Ein in vielen Anwendungen der Nichtlinearen Programming sehr effizientes Verfahren ist Sequential Quadratic Programming (SQP), ein spezielles Newton-Verfahren. Eine Übersicht zur Theorie von SQP-Verfahren findet sich bei Boggs und Tolle (1995). Die Grundidee von SQP ist, dass in jeder Iteration ein quadratisches Subproblem mit linearen Randbedingungen gelöst wird, um einen passenden Suchschritt $\underline{d}=\underline{x}-\underline{x}^{k}$ zu finden. Das Subproblem wird im Allgemeinen abgeleitet, indem man in der Nähe des Minimums eine Taylor-Approximation zweiter Ordnung bildet und die notwendige Bedingung für einen Extremwert der Zielfunktion anwendet, d.h. der Gradient der Lagrange-Funktion muss verschwinden. Das resultierende nichtlineare Gleichungssystem entspricht nun einem quadratischen Problem:

$$
\begin{gathered}
\text { Minimiere } \\
\left(\underline{r}^{k}\right)+\frac{1}{2}(\underline{d})^{T} \underline{\underline{B}}^{k} \underline{d}
\end{gathered}
$$


mit den Randbedingungen

$$
\begin{array}{ll}
\underline{\nabla} h_{i}\left(\underline{x}^{k}\right)^{T} \underline{d}+\underline{\nabla} h_{i}\left(\underline{x}^{k}\right)=0 & i=1,2 \ldots, I \\
\underline{\nabla} g_{j}\left(\underline{x}^{k}\right)^{T} \underline{d}+\underline{\nabla} g_{j}\left(\underline{x}^{k}\right) \geq 0 & j=1,2 \ldots, J
\end{array}
$$

Der Vektor $\underline{r}^{k}$ und die positiv definite Matrix $\underline{\underline{B}}^{k}$ müssen zunächst bestimmt werden. Hierfür sind verschiedene Optionen vorhanden und unterscheiden sich von SQP- zu SQP-Algorithmus. Eine Option ist hierzu zum Beispiel für $\underline{r}^{k}$ den Gradient $\underline{\nabla} f$ und für die Matrix $\underline{B}^{k}$ eine Approximation der Hessischen Matrix der Lagrange-Funktion auszuwählen (siehe Schittkowski (1992)). Bei diesem Optimierungsproblem ist die Suchrichtung $\underline{d}$ der Vektor der freien Variablen. Mit den aus Gl. (4.3) gewonnenen Lösung $\underline{d}^{k}$ werden dann später die freien Variablen $\underline{x}$ des Optimierungsproblems aktualisiert.

Da die Optimierungsaufgabe (4.3) eine quadratische Zielfunktion enthält, wird sie nach der so genannten Quadratic Programming (QP) Methode gelöst (z.B. Gill et al., 1982). QP ist selbst ein iteratives Verfahren und wird oft bei SQP minor iteration genannt. Die QP Probleme sind im Vergleich zum ursprünglichen Optimierungsproblem einfacher zu lösen. Zur Sicherung und Beschleunigung der Konvergenz wird die Schrittweite $\alpha$ selbst als Lösung eines Optimierungsproblems ermittelt. Dadurch wird in jedem Schritt eine genügende Abnahme der Zielfunktion garantiert.

Die Aktualisierung (update) der Matrix $\underline{\underline{B}}^{k}$ für den nächsten Schritt $\underline{\underline{B}}^{k+1}$ ist der andere wichtige Teil des SQP Algorithmus. Die Berechnung der Hessischen Matrix stellt den aufwendigsten Teil dar, besonders wenn die zweiten Ableitungen numerisch approximiert werden müssen und die Auswertung von $f$ aufwendig ist. Deshalb werden relativ einfache Methoden eingesetzt, die nur Informationen von dem vorherigen Schritt verwenden, nämlich die Suchrichtung $\underline{d}^{k}$, die Gradienten der Lagrange-Funktion und die Matrix $\underline{B}^{k}$ selbst. Die meisten Algorithmen verwenden die Broyden-Fletcher-GoldfarbShanno (BFGS) Formel. Bei der Approximation muss die Hessische Matrix $\underline{\underline{B}}^{k}$ positiv definit sein. Dies ist nicht immer garantiert, wenn der aktuelle Vektor weit von einem Minimum entfernt ist. Spezielle Verfahren werden in die SQP-Algorithmen eingeführt, um die Matrix $\underline{\underline{B}}$ notfalls positiv definit zu machen.

In vielen hydrodynamischen Optimierungsproblemen liegt ein analytisch geschlossenes Modell der Zielfunktion nicht vor. Deshalb müssen die Ableitungen der Zielfunktion durch numerische Differenziation approximiert werden. In einfachster Form (forward differences) werden mindestens $n+1$ Funktionsauswertungen benötigt, um die Gradienten der Zielfunktion mit $n$ freien Variablen abzuschätzen. Bei fast allen Gradientenverfahren ist eine ausreichende Genauigkeit in der Auswertung der Zielfunktion die Voraussetzung für den Erfolg des Algorithmus.

In den letzten Jahren hat sich Sequential Quadratic Programming (SQP) als eine Art Standardverfahren zur Lösung von Optimierungsproblemen etabliert und ist Bestandteil numerischer Unterprogrammbibliotheken und Mathematikprogramme. Verschiedenste SQP-Implementierungen existieren, die sich hinsichtlich der Approximation der Hessischen Matrix, der Lösung des quadratischen Subproblems und der Schrittweitensteuerung unterscheiden (z.B. Gill et al., 1982, 1992; Fletcher, 1987; Schittkowski, 1992). Im Rahmen dieser Arbeit wird der SQP-Algorithmus nach Schittkowski (1985) 
verwendet, der auch ein Teil der MATLAB Optimierung-Toolbox und anderer numerischer Bibliotheken ist.

Die lokalen Algorithmen können auch für multimodale Zielfunktionen verwendet werden. Zum Beispiel kann der SQP-Algorithmus mit unterschiedlichen Startpunkten mehrmals gestartet werden, um möglichst viele Minima zu lokalisieren. Da SQP schnell in das nächstgelegene Minimum konvergiert, kann das globale Minimum effizient gefunden werden, wenn ein Startvektor in der Nähe des globalen Optimums liegt. Zur Lösung des multimodalen Optimierungsproblems stellte Nowacki (2003) generell folgende Methoden vor:

- Mehrfache Startpunkte an der Ecke des zulässigen Lösungsraumes

- Regionale Aufteilung des zulässigen Raumes mit Hilfe der Information über die Konvexität bzw. Konkavität der Nebenbedingungen

- Anwendung von Zufallsmethoden mit großer Standardabweichung

Ein günstiger Startvektor kann durch eine Problemanalyse der Optimierungsaufgabe gewonnen werden, bei der die Informationen über das Gebirge des Lösungsraumes und über die Art der Nebenbedingungen (konvex oder konkav) ausgenutzt werden. In der Regel ist der Rechenaufwand für die Berechnung der Zielfunktion bei der technischen Anwendung sehr groß. Es ist deshalb schwierig, einen erfolgversprechenden Startvektor vor der Optimierung zu bestimmen, vor allem wenn die Anzahl der freien Variablen und der Nebenbedingungen groß wird. Für den Fall einer pathologisch mutimodalen Zielfunktion (z.B. Fahnenmasten) sind keine der oben genannten Methode sicher.

In der vorliegenden Arbeit wird das SQP-Verfahren mit den globalen Optimierungsalgorithmen zu einem hybriden Verfahren kombiniert und bei der hydrodynamischen Formoptimierung verwendet. Im Allgemeinen konvergieren globale Optimierungsalgorithmen langsam zum globalen Optimum. Zur Beschleunigung der Optimierung bietet sich an, die durch globale Optimierungsalgorithmen gefundenen potentiellen Minima als Startvektor einer lokalen Strategie zu verwenden, die schnell in das nächstgelegene Minimum konvergiert.

\subsection{Globale Optimierungsstrategien}

Im folgenden werden drei Klassen von globalen Optimierungsstrategien vorgestellt, die im Rahmen dieser Arbeit vergleichend eingesetzt werden:

- Genetische Algorithmen bzw. Evolutionsstrategien,

- Simulated Annealing und

- Branch-and Bound Verfahren.

Bei allen drei Algorithmen existiert eine Vielzahl von Varianten. Die ersten beiden Klassen repräsentieren stochastische Methoden, die in der Natur auftretende Vorgänge nachahmen. Die letzte Klasse gehört dagegen zur Gruppe der deterministischen 
Verfahren und ist diskret-mathematisch orientiert. In den folgenden Abschnitten werden die Grundlagen dieser globalen Optimierungsstrategien kurz beschrieben, um ihre Funktionsweise bei technischen Optimierungsaufgaben zu illustrieren.

\subsubsection{Genetische Algorithmen (GA)}

Genetische Algorithmen beruhen auf den drei Prinzipien der biologischen Evolution:

- Mutation

- Fortpflanzung bzw. Rekombination und

- Selektion

Der wesentliche Motor der Evolution ist die Bewährung eines Individumms in seiner Umwelt und Konkurrenzkampf zu anderen Arten oder Artgenossen, das sogenannte Survival of the Fittest (Überleben des Geeignetsten). Zugrunde liegt die Beobachtung, dass sich besser geeignete Individuen einer Spezies mit größerer Wahrscheinlichkeit überleben und sich fortpflanzen. Bezogen auf eine technische Optimierung heißt das, dass die besten Entwürfe (Individuen) behalten werden und aus ihnen neue Entwürfe erzeugt werden, während die schlechteren Entwürfe verworfen werden (Aussterben). Was ein guter und was ein schlechter Entwurf ist, wird anhand der Zielfunktion entschieden, die im GA-Sprachgebrauch auch Fitness genannt wird.

Die Individuen einer Generation werden mit Hilfe der beiden anderen Prinzipien erzeugt. Rekombination bezeichnet den Austausch von Genen bei der geschlechtlichen Fortpflanzung der Eltern, die die Selektionsstufe überstanden haben (gute Entwürfe). Bei der Lösung von Optimierungsaufgaben wird dies durch Austausch der Werte von freien Variablen (Genen) realisiert. Die Gesamtheit aller Informationen über ein Individuum wird bei GA als Genotyp bezeichnet. Das Pendant in der technischen Optimierung ist der Vektor der freien Variablen.

Es gibt viele Möglichkeiten, wie aus der gesamten Population die für das Crossover bestimmten Individuen ermittelt werden können. Eine Möglichkeit ist, ein imaginäres Roulette-Rad zu bilden, auf der jedes Individuum so viel Platz hat wie seine Fitness im Verhältnis zur Gesamtfitness (Summe aller Einzelfitnesswerte). Dann lässt man diesen Roulette-Teller drehen und ermittelt so jeweils zwei Elternindividuen (probable selection). Eine bessere und heute öfter verwendete Methode ist eine Rangliste aufzustellen, in der die Individuen nach ihrer Fitness angeordnet werden. Daraufhin wird eine festgelegte Anzahl von zufällig ausgewählten Individuen gezogen und davon der beste für die Rekombination bestimmt (tournament selection). Dabei lässt sich durch die Größe dieser Gruppe der Selektionsdruck kontrollieren, mit der sich ein gutes Individuum gegenüber schlechteren durchsetzt. Je größer die Gruppe angelegt wird, desto schneller setzen sich Individuen mit hoher Fitness durch. Dadurch kommt es nur auf die relative Position in der Rangliste an, und auf eine absolute Skalierung kann verzichtet werden. Details über den Selektionsprozess findet sich in Goldberg (1989) und in Michalewicz (1994). 
Wäre die Rekombination die einzige Möglichkeit, neue Individuen zu erzeugen, könnten im Laufe der Evolution (Optimierung) nur Gene (Werte freier Variablen) auftreten, die bereits in der ersten Generation vorhanden sind (sozusagen Inzucht). Diese Einschränkung wird durch das Prinzip der Mutation durchbrochen. Eine Mutation bewirkt eine Änderung eines zufällig ausgewählten Gens bzw. einer freien Variablen. Durch Einsatz einer Wahrscheinlichkeitsdichteverteilung (z.B. Gauß-Verteilung mit Mittelwert $\mu=0$ ) werden große Änderungen weniger wahrscheinlich als kleine. In jeder Generation wird ein kleiner Prozentsatz der durch Rekombination erzeugten neuen Entwürfe mehr oder weniger stark durch Mutation in seinen Eigenschaften verändert, um Stillstand zu verhindern. In den ursprünglichen GA wurden die freien Variablen als binäre Zahlen kodiert. Dies erwies sich jedoch als unvorteilhaft für technische Probleme, bei denen freie Variablen meist reelle Zahlen sind. Inzwischen gibt es, wie bei Evolutionsstrategien schon immer üblich, auch reellwertige Codierung der freien Variablen bei Genetischen Algorithmen (Michalewicz, 1994).

Die Optimierung (Evolution) startet nicht mit einer einzigen Ausgangsstruktur, sondern mit einer Menge (Population) zufällig generierter Entwürfe aus dem Variablenraum. Für alle Entwürfe dieser ersten Generation wird die Zielfunktion ausgewertet, d.h. die Seegangseigenschaften berechnet und anhand dieses Fitness-Maßstabes die Eltern der nächsten Generation selektiert. Durch Rekombination und Mutation wird aus den Eltern die folgende Generation erzeugt. In manchen GA-Implementierungen werden die besten Entwürfe der Elterngeneration mit in die Kindergeneration übernommen, d.h. sie leben länger. Bewertung der neuen Entwürfe und anschließende Selektion erzeugen wiederum neue Eltern für die übernächste Entwurfsgeneration. Meist wird die Optimierung beendet, wenn sich der Mittelwert der Güte der Entwürfe einer Generation über mehrere Generationen hinweg nicht mehr merklich ändert.

Bei den unzähligen Varianten Genetischer Algorithmen, die in den letzten zwanzig Jahren entstanden sind, fällt es schwer, den Überblick zu behalten. Verschiedene Methoden der Selektion, Rekombination und Mutation wurden implementiert und an Beispielen getestet. Eine allgemeingültige Aussage, welche Methoden für welche Aufgabenstellung am besten geeignet sind, kann es nicht geben. Auch fehlt für Genetische Algorithmen der Nachweis, dass sie gegen das globale Optimum konvergieren. Durch ausgiebige Tests müssen die Parameter der verwendeten Implementierung an die eigene Aufgabenstellung angepasst werden. Man kann dies durchaus als Optimierung der Optimierung bezeichnen und auch hierfür kann es automatisierte Verfahren geben.

Bei der hydrodynamischen Formoptimierung wird ein genetischer Algorithmus nach Houck et al. (1995b) eingesetzt, bei dem die freien Variablen direkt als reellwertige Vektoren eingegeben werden können. Der Algorithmus verwendet die sogenannte RankingMethode für die Selektion der Individuen. Zur Mutation wird als Steuerparameter Non-Uniform Mutation ausgewählt.

\subsubsection{Simulated Annealing (SA)}

Simulated Annealing bildet das langsame Abkühlen einer Schmelze nach, bei dem durch kontrollierte Wärmezufuhr die Bildung perfekter atomarer Strukturen (z.B. bei großen Kristallen) erzielt werden soll. Während des langsamen Abkühlens eines Metallstückes 
ordnen sich die einzelnen Atome so an, dass sie einen Zustand möglichst niedriger Energie einnehmen. Ein optimaler Kristall besitzt eine Konfiguration geringster Energie. Wird zu schnell abgekühlt, erreichen die Kristalle nicht die optimale, energieärmste Konfiguration. Ein Fehlstelle kann man sich als Atom vorstellen, das in einer lokalen Energiemulde festsitzt. Werden perfekte Kristalle benötigt, so muss man sie in einem aufwendigen Verfahren herstellen: Sie werden sehr langsam, mit kontrollierter Wärmezufuhr abgekühlt. Die partiell zugeführte Energie erlaubt es den in den Mulden festsitzenden Atomen, die energetischen Barrieren zu überwinden und ihre korrekten Gitterplätze geringster Energie zu erreichen. Die Grundidee der Abkühlung wurde von Kirkpatrick et al. (1983) und Cerny (1985) unabhängig voneinander auf Optimierungsprobleme übertragen.

Die Temperatur $T_{k}$ übernimmt in der Optimierung die Steuerung von Wahrscheinlichkeiten, die die Änderungen der freien Variablen beeinflussen. In jedem Optimierungsschritt $k$ wird ein neuer Entwurf durch eine zufällige Variation des Vektors der freien Variablen erzeugt. Die Schrittweiten der freien Variablenänderung sind von einer Wahrscheinlichkeitsdichtefunktion, der so genannten Erzeugungsfunktion, und den Unter- und Obergrenzen der freien Variablen abhängig. Die Erzeugungsfunktion wird im Laufe der Optimierung mit sinkender Temperatur $T_{k}$ so verändert, dass die Wahrscheinlichkeiten großer Schrittweiten immer kleiner werden. Eine geringe Wahrscheinlichkeit für große Sprünge im Variablenraum ist immer noch vorhanden, und ermöglicht das Verlassen lokaler Minima. Der Wert der Zielfunktion des veränderten Entwurfs wird mit dem Original verglichen und im Falle einer Verbesserung akzeptiert.

Im Gegensatz zu lokalen Verfahren, werden bei SA-Algorithmen jedoch auch schlechtere Zielfunktionswerte als Ausgangsbasis des nächsten Schrittes akzeptiert: Anhand einer Akzeptanzfunktion und einer Zufallszahl wird entschieden, ob der neue Entwurf übernommen wird (Zufallszahl kleiner oder gleich der Akzeptanzfunktion) oder nicht (Zufallszahl größer als Akzeptanzfunktion). Auch die Akzeptanzfunktion wird mit sinkender Temperatur $T_{k}$ so verändert, dass die Wahrscheinlichkeit, einen schlechteren Entwurf als Basis zu übernehmen, kleiner wird. Am Ende des Optimierungsschritts wird die Temperatur für den nächsten Schritt abgesenkt $\left(T_{k+1}<T_{k}\right)$ und eine neue Variablenvariation durchgeführt. Das Verfahren endet, wenn die vorgegebene minimale Temperatur erreicht wird.

Durch die Möglichkeit, auch schlechtere Funktionswerte beizubehalten, wird die Gefahr, in lokalen Minima stecken zu bleiben, zusätzlich reduziert. Da der ursprüngliche SA-Algorithmus von Kirkpatrick et al. (1983) nur sehr langsam konvergiert, wurden von verschiedenen Autoren Varianten mit rascherer Temperaturabsenkung und besserer Konvergenz entwickelt. Große Verbreitung fanden die Arbeiten von Ingber (1989), dessen Adaptive Simulated Annealing (ASA) Algorithmus auch im Rahmen dieses Projektes verwendet wird (Ingber, 1993, 1996). Der ASA Algorithmus erlaubt die Berücksichtigung von Nebenbedingungen. Für die entarteten Geometrien und die Entwürfe, die eine Nebenbedingung verletzen, verwirft ASA solche Kombinationen freier Variablen. 


\subsubsection{Angepasste Branch-and-Bound Algorithmen (BaB)}

BaB-Algorithmen beginnen mit dem Lösen eines sogenannten root-Problems, das durch die Unterschätzung der Zielfunktion erstellt wird (Relaxation, Abb 4.1 (a)). Die Lösung des root-Problems liefert eine untere Grenze $L_{R}$ für die Zielfunktion und wird als Anfangspunkt für lokale Algorithmen verwendet, um eine obere Grenze $U_{R}$ für das globale Optimum zu bestimmen (Abb 4.1 (a)). Ist die Differenz $\left(U_{R}-L_{R}\right)$ nicht klein genug, wird der zulässige Lösungsraum in kleinere Abschnitte $\left(R_{1}, R_{2}\right)$ aufgeteilt (Branching, Abb.4.1 (b)), die zur weiteren Bearbeitung in einer Liste abgespeichert werden. Für jeden Abschnitt wird eine untere und obere Grenze der Zielfunktionswerte abgeschätzt (Bounding, Abb.4.1 (c)). Durch den Vergleich der unteren und oberen Schranken unterschiedlicher Abschnitte, können alle Abschnitte, deren untere Grenze größer ist als die obere Schranke anderer $\left(L_{2}>U_{1}\right)$, von der weiteren Suche ausgeschlossen werden (Cut-Off-Test, Abb.4.1 (c)). Solche Abschnitte werden fathomed (ausgelotet) bezeichnet. Der abzusuchende Lösungsraum wird dadurch schnell und deutlich verkleinert. Abschnitte, die nicht durch den Cut-Off-Test von der Bearbeitungsliste entfernt wurden, werden erneut aufgeteilt $\left(R_{1 a}, R_{1 b}\right)$ und die entstehenden Abschnitte werden in die Bearbeitungsliste eingefügt. Bei Optimierungen mit kontinuierlichen Variablen wird der Prozess beendet, wenn das Intervall $\left(U_{1 a}-L_{1 a}\right)$ mit dem besten (globalen) Minimum eine vorgegebene Größe unterschreitet, bzw. die obere Schranke für das globale Optimum mit der unteren Schranke eines Abschnittes zusammenfällt (Abb.4.1 (d)).

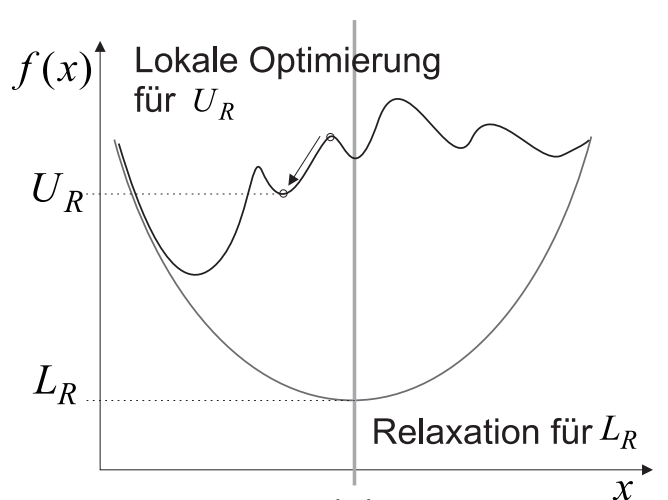

(a)

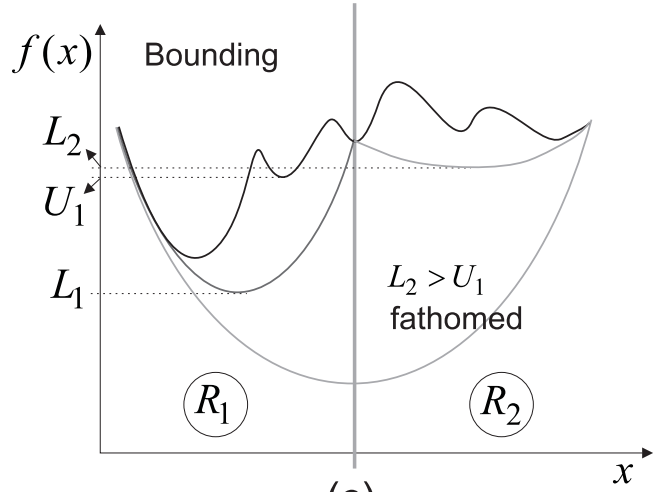

(c)

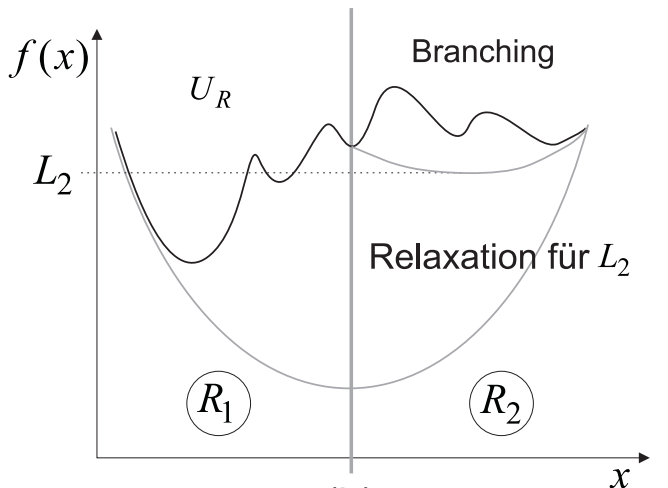

(b)

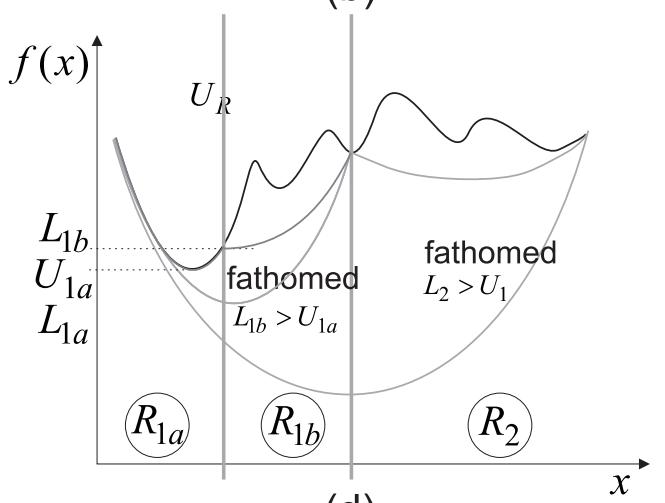

(d)

Abbildung 4.1: Grundsätze der Branch-and-Bound Methoden 
In die hydrodynamiche Formoptimierung wird ein MINLP (Mixed Integer Nonlinear Programming) Algorithmus eingesetzt (Kuipers, 2000), der auf den BaB Algorithmen basiert und sowohl für kontinuierliche als auch ganzzahlige Variable anwendbar ist (Grossman, 1990). Der MINLP Algorithmus beginnt mit einem ersten Subproblem, wobei ganzzahlige als kontinuierliche Variable betrachtet werden. Daraus wird ein kontinuierliches NLP (Nonlinear Programming) Problem gebildet, das durch den lokalen Algorithmus SQP gelöst wird. Danach werden durch Branching Teilbäume erzeugt, die nacheinander eine Untermenge der ganzzahligen Variablen haben. Diese Subprobleme werden wiederum wie ein kontinuierliches NLP Problem gelöst, um die untere Grenze für die Zielfunktion zu bestimmen. Da bei der Formoptimierung nur kontinuierliche freie Variable verwendet werden, muss für den Einsatz des MINLP Algorithmus mindestens eine freie Variable in eine Ganzzahlige umgewandelt. Der Hauptnachteil von MINLP Algorithmen ist, dass viele Lösungen des NLP-Subproblems benötigt werden.

\subsection{Verhalten bei multimodalen Zielfunktionen}

Um das Konvergenzverhalten der Optimierungsalgorithmen bei multimodalen Zielfunktionen zu untersuchen, wurden die lokalen und globalen Optimierungsalgorithmen an einer analytischen, multimodalen Funktion getestet. Als Testfunktion wurde die Griewank-Funktion (Törn und Zilinskas, 1989) ausgewählt.

$$
f(\mathbf{x})=\frac{x_{1}^{2}+x_{2}^{2}}{200}-\cos \left(x_{1}\right) \cos \frac{x_{2}}{\sqrt{2}}+1
$$

Sie hat zwei unabhängige freie Variablen $\mathbf{x}=\left(x_{1}, x_{2}\right)$, was die graphische Darstellung der Optimierungsabläufe ermöglicht. Abb. 4.2 zeigt das Funktionsgebirge, das viele lokale Minima und ein globales Minimum bei $\mathbf{x}=(0,0)$ mit $f(\mathbf{x})=0$ aufweist. Die Optimierungsstrategien sollten von einem beliebigen Startpunkt ausgehend in möglichst wenigen Schritten das globale Optimum auffinden.

In den folgenden Tests soll anhand dieser analytischen Funktion demonstriert werden, wie sich die unterschiedlichen Optimierungsalgorithmen bei der Suche nach dem besten Minimum im multimodalen Parameterraum verhalten.

\subsubsection{Sequential Quadratic Programming (SQP)}

Wie zu erwarten war, findet das lokale SQP-Verfahren in der Regel nur das dem Startpunkt nächstgelegene lokale Minimum. Als Beispiel zeigt Abb. 4.3, wie sich die SQPMethode mit dem Startvektor $(-5,6)$ nach dem 20. Optimierungsschritt einem lokalen Minimum $f=0.14$ mit $\mathbf{x}=(-3.11,4.36)$ nähert, das in der Nähe des Startpunktes liegt. Bei unterschiedlichen Anfangsparametern liefert die SQP-Methode andere lokale Minima. Liegt der Anfangsvektor der freien Variablen $\mathbf{x}=\left(x_{1}, x_{2}\right)$ zufällig in der Nähe des globalen Minimums, so findet die Methode auch das gesuchte Optimum. 

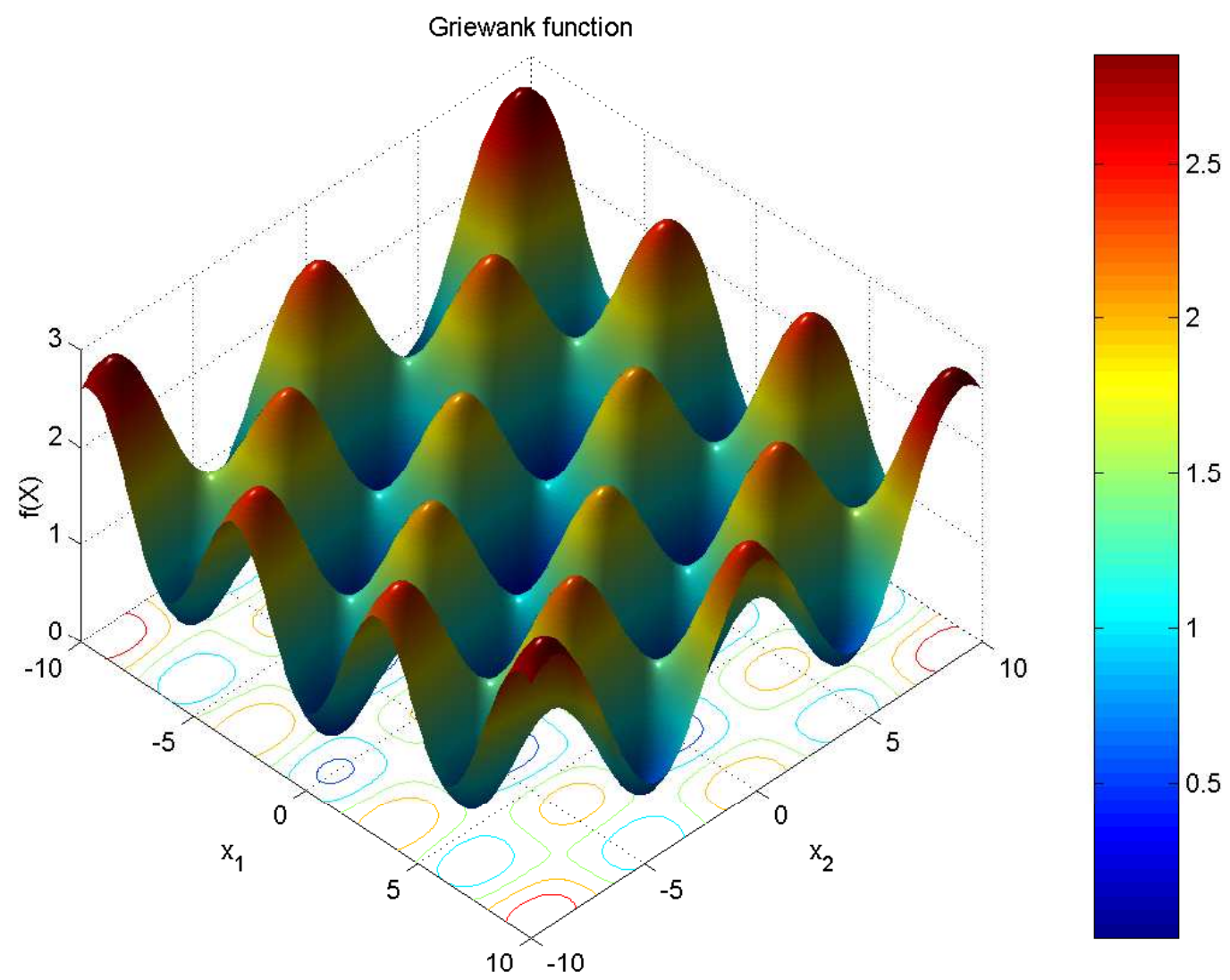

Abbildung 4.2: Eine analytische, multimodale Funktion mit 2 Variablen

\subsubsection{Adaptive Simulated Annealing (ASA)}

Im Gegensatz zu den lokalen Optimierungsstrategien konnte bei Anwendung von $A d$ aptive Simulated Annealing (ASA) das globale Minimum erfolgreich gefunden werden (Abb. 4.4). Dazu benötigt das stochastische Suchverfahren jedoch sehr viele Funktionswertberechnungen. Es ist ein starkes Oszillieren der Variablen und der Werte der Zielfunktion zu beobachten. Wie in Abb. 4.4 die Zielfunktionswerte in Abhängigkeit des Optimierungsschrittes zeigen, nähert sich das ASA-Verfahren bereits nach dem hundertsten Schritt dem globalen Minimum bei $\mathbf{x}=(0,0)$. Da die Kontrolltemperatur $T$ für die Konvergenz der Lösung noch nicht weit genug abgesunken ist, sucht das Verfahren mit starken Sprüngen weiter nach besseren Lösungen. Dies läßt vermuten, dass sich durch problemspezifische Anpassungen der Steuerparameter (Akzeptanzwahrscheinlichkeit, Genauigkeit des Zielfunktionswertes etc.) noch Reduzierungen in der Zahl der Optimierungsschritte erzielen lassen. Bei allen Testläufen findet das Simulated Annealing das globale Minimum. 

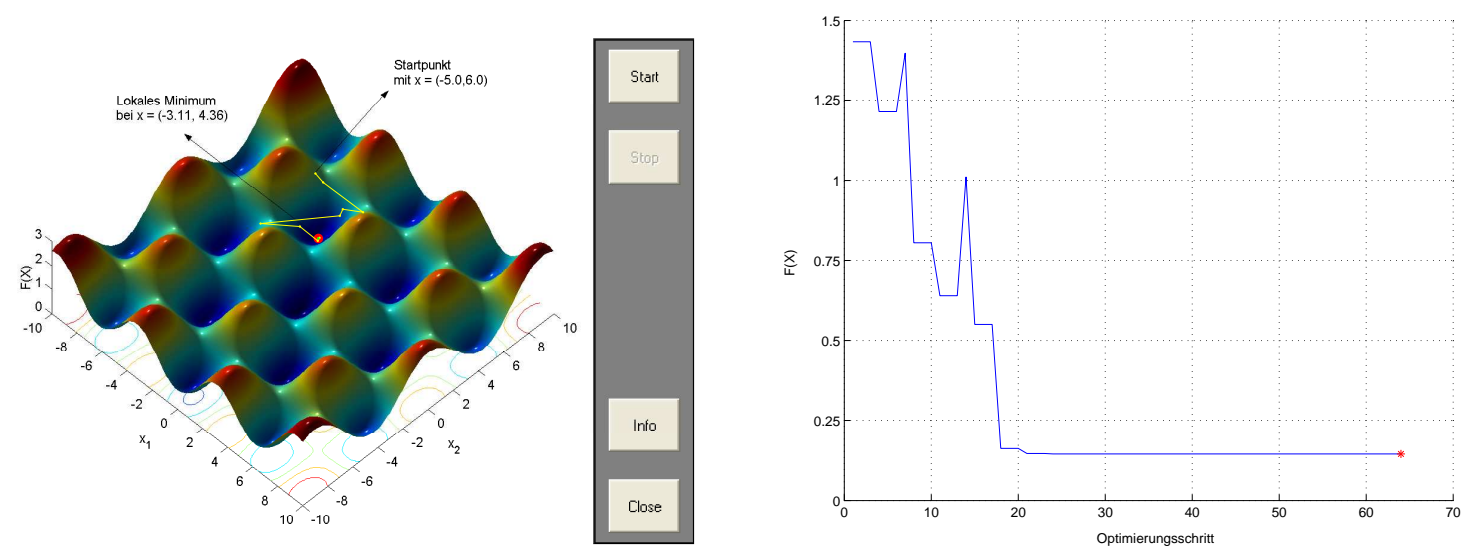

Abbildung 4.3: Minimierung einer Griewank-Funktion unter Anwendung des Sequential Quadratic Programming (lokale Suchstrategie).
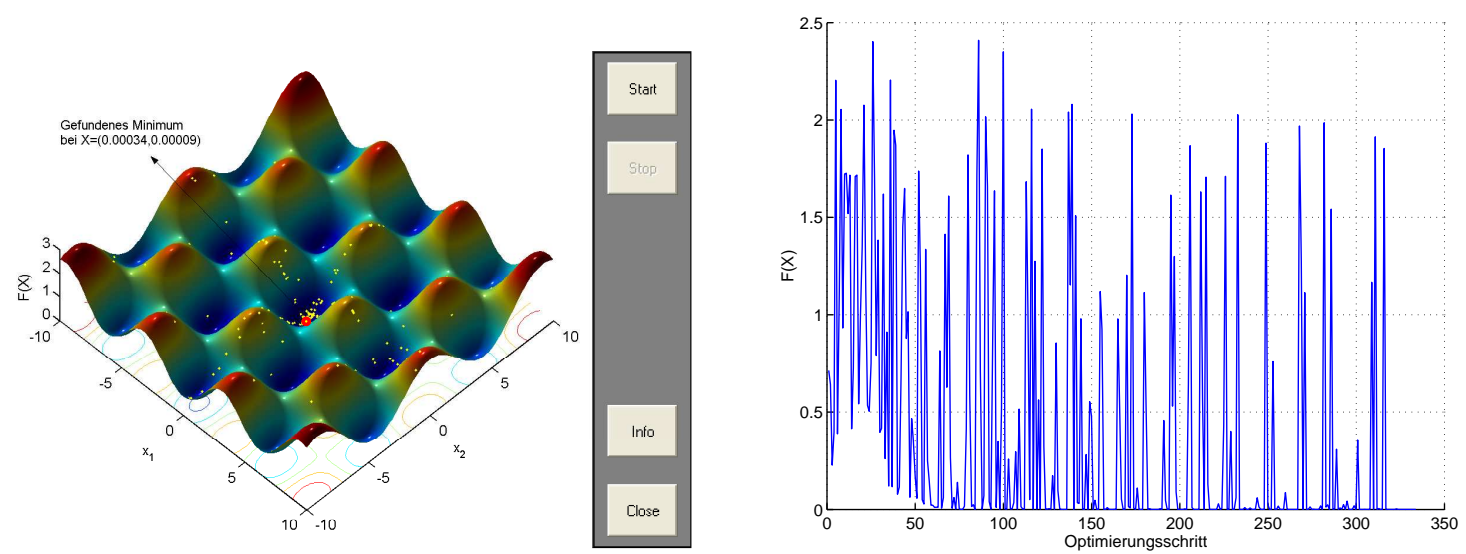

Abbildung 4.4: Minimierung der Griewank-Funktion unter Anwendung von Adaptive Simulated Annealing (ASA, globale Optimierungsstrategie)

\subsubsection{Genetische Algorithmen (GA)}

Wie Abb. 4.5 zeigt, findet auch der Genetische Algorithmus (GA) das globale Optimum. Die Streuung der freien Variablenwerte ist dabei deutlich größer als beim Adaptive Simulated Annealing. Das Ergebnis der Optimierung hängt bei GA von der maximalen Anzahl der zulässigen Generationen ab. Je höher, desto größer die Wahrscheinlichkeit, das globale Optimum zu treffen. Bei dem abgebildeten Beispiel findet GA nur in 60 $\%$ der Testläufe das globale Optimum, trotz gleicher Steuerparameter (z.B. Größe der Anfangspopulation, Parameter für Mutation und Rekombination etc.). Tendenziell werden mehr Auswertungen der Zielfunktion benötigt als beim Adaptive Simulated Annealing und die Wahrscheinlichkeit das globale Optimum zu finden ist für diese Testfunktion bei GA kleiner. 

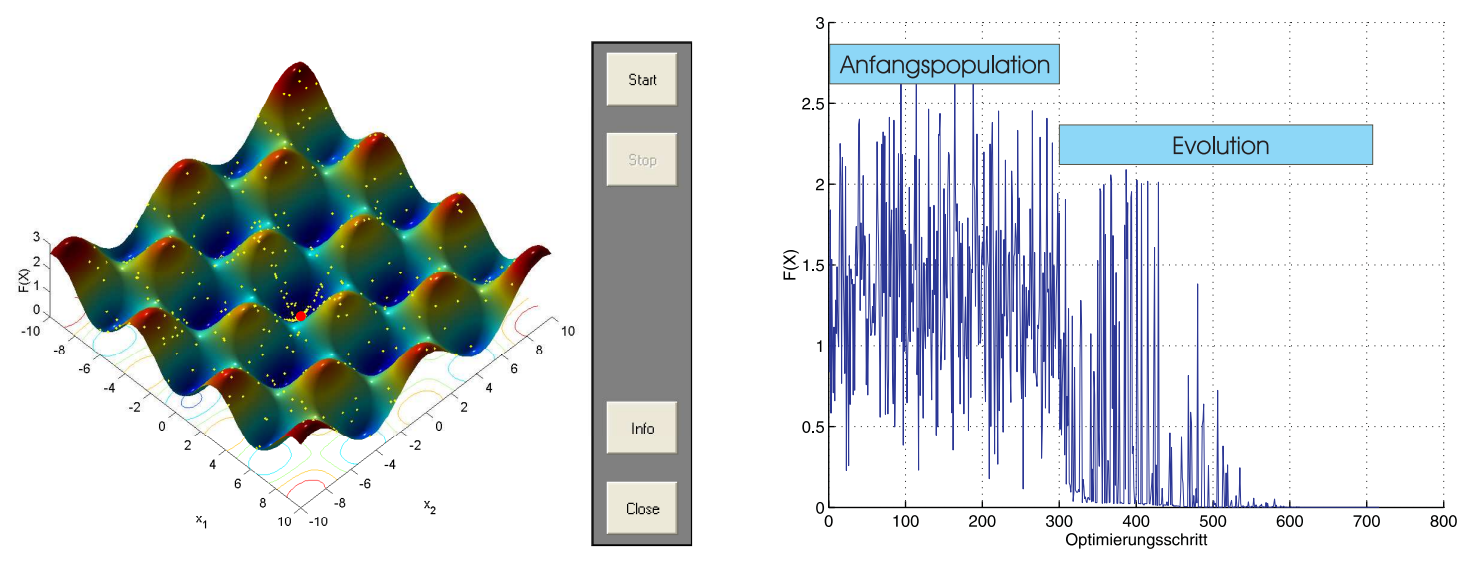

Abbildung 4.5: Minimierung der Griewank-Funktion unter Anwendung des Genetischen Algorithmus (GA, globale Optimierungsstrategie)

\subsubsection{Hybride Optimierung}

Wie das Beispiel aus Abb. 4.4 deutlich macht, trifft das Simulated Annealing bereits nach 100 Schritten das globale Optimum. Dieses wird dann allerdings noch nicht als solches erkannt, weil die Konvergenzkriterien noch nicht erfüllt sind. Es bietet sich an, solch potenzielle Optima als Startvektor einer lokalen Strategie zu verwenden, die schnell in das nächstgelegene Optimum konvergiert. Vor diesem Hintergrund wurde das ASA-Verfahren mit dem SQP-Verfahren zu einem hybriden Verfahren kombiniert und auf die Griewank-Funktion (Gl. 4.4) angewandt (siehe Abb. 4.6). In Schritt 61 findet ASA ein potentielles Optimum bei $x=(-0.18,0.4063)$, das als Anfangsvektor der lokalen Suchstrategie SQP verwendet wird. Das SQP-Verfahren findet nach wenigen Optimierungsschritten das globale Optimum mit hohen Genauigkeit $\left(1 e^{-12}\right)$, da das ASA-Verfahren bereits in der Nähe des Optimums konvergiert war. Im Vergleich zu der Anwendung mit dem ASA-Verfahren allein zeigt dieses Ergebnis, dass beim Auffinden des globalen Optimums eine deutliche Beschleunigung der Optimierung durch die gezielte Kombination des SQP-Verfahren mit dem ASA-Verfahren erzielt werden kann.

Abb. 4.7 zeigt als weiteres Beispiel einer hybriden Optimierung die Minimierung der Griewank-Funktion unter Anwendung von GA und SQP. Aus 300 zufällig verteilten Anfangsindividuen liefert GA-Verfahren ein potenzielles Minimum, das sich nach 10 Generationen als "bestes Individuum" auszeichnet. Mit diesem Anfangsvektor findet das SQP-Verfahren das gesuchte globale Optimum. Die Anzahl der notwendigen Schritte wurde gegenüber der Anwendung des reinen Genetischen Algorithmus um ca. $40 \%$ gesenkt.

Beide Ergebnisse bestätigen, dass der Rechenaufwand bei globaler Optimierung durch den gezielten Einsatz von hybriden Optimierungsmethoden reduziert werden kann. Allerdings bleibt die Gefahr, das tatsächliche Optimum zu verfehlen, da globale Algorithmen nicht immer einen Erfolg versprechenden Anfangsvektor für die lokale, deterministische Suchstrategie ermitteln können. 


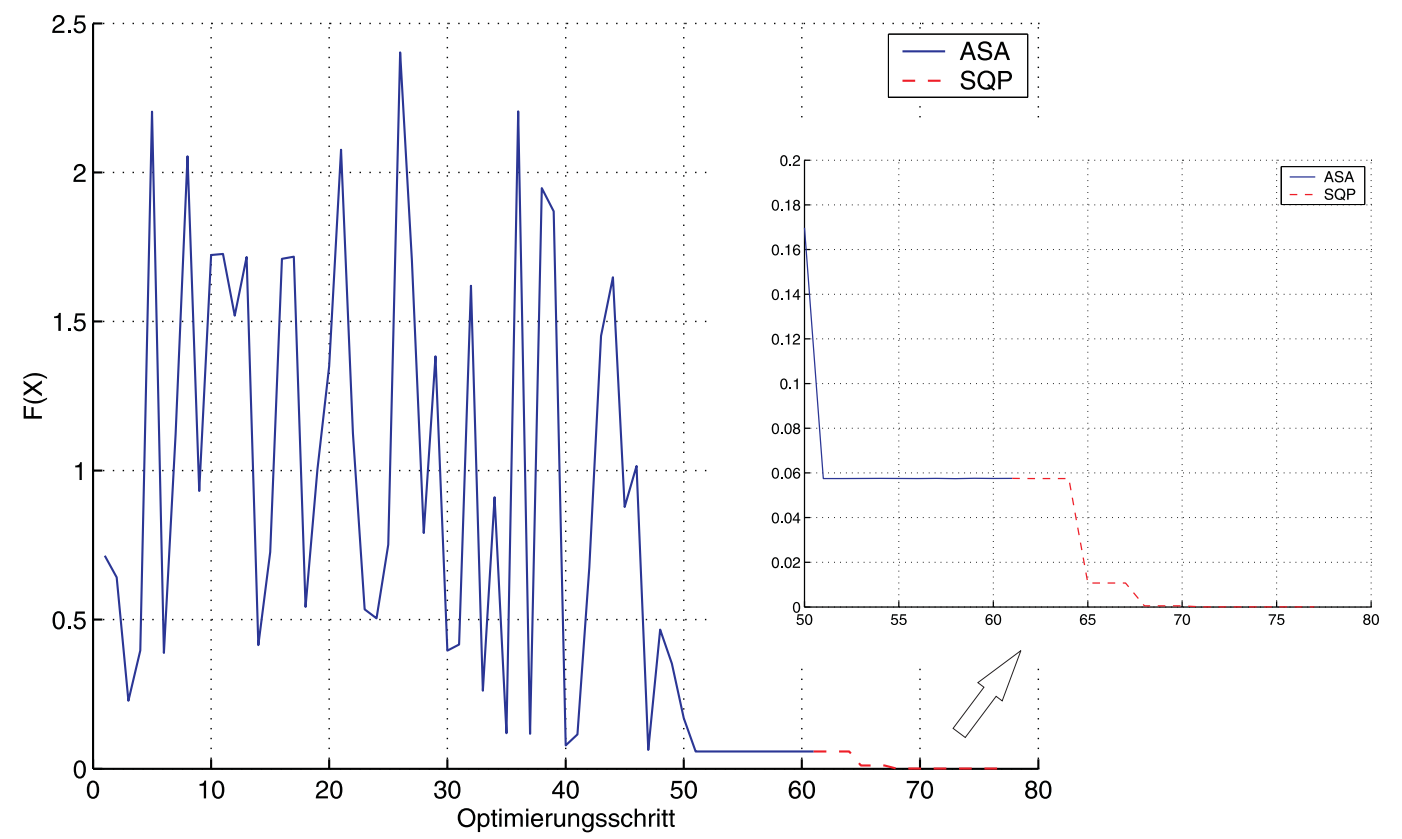

Abbildung 4.6: Minimierung der Griewank-Funktion unter kombinierter Anwendung von ASA und SQP-Methode (Hybride Optimierungsmethode)

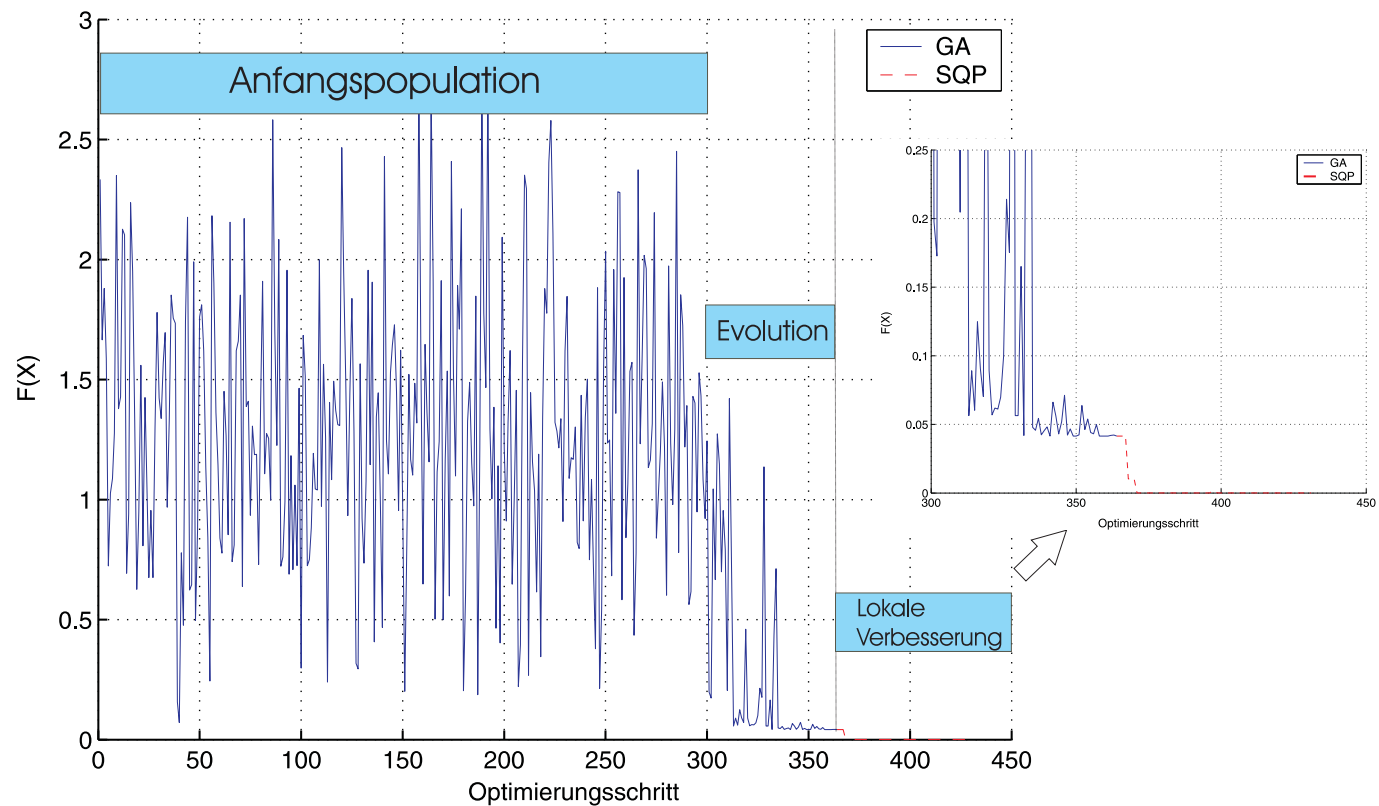

Abbildung 4.7: Minimierung der Griewank-Funktion: kombinierte Anwendung von GA und SQP-Methode (Hybride Optimierungsmethode) 


\section{Kapitel 5}

\section{Definition und Berechnung der Zielfunktionen}

Die automatische, durch Formparameter gesteuerte, Geometrieerzeugung ist eine notwendige Voraussetzung für die Durchführung der Formoptimierung. Im ersten Abschnitt wird deshalb zunächst das Verfahren der Geometriemodellierung beschrieben. Für die Lösung von Optimierungsaufgaben ist die Festlegung der Zielfunktion und des für ihre Berechnung notwendigen mathematischen Modells von grundlegender Bedeutung, da die Ergebnisse der Optimierung von den Eigenschaften des Modells der Zielfunktion abhängen. Im Abschnitt 5.2 wird kurz die hydrodynamische Analyse beschrieben, wobei die Kräfte und Bewegungen der Konstruktionen im Seegang ermittelt werden. Die theoretischen Grundlagen der hydrodynamischen Analyse werden im Anhang $\mathrm{B}$ und $\mathrm{C}$ vorgestellt. Anschließend werden im Abschnitt 5.3 die in dieser Arbeit verwendeten Zielfunktionen und deren Berechnungen erläutert.

\subsection{Geometriemodellierung}

Im rechnergestützten Schiffsentwurf werden Schiffskörper aus Grundlinien (z.B. Wasserlinien, Spantarealkurve, usw.) erzeugt (Nowacki, 1994; Nowacki et al., 1995). Meerestechnische Konstruktionen besitzen in der Regel nicht die bei Schiffen üblichen komplexen Freiformflächen, sondern sie bestehen vielmehr aus mehreren Komponenten mit einfachen Grundformen (Zylinder, Quader, etc.). Auch bei meerestechnischen Konstruktionen kann eine Modellierung für je eine Strukturkomponente auf der Basis sogenannter Grundkurven eingesetzt werden, wie sie im Prinzip schon von Taylor zur Erzeugung seiner Serienmodelle verwendet wurde (Taylor, 1915). Drei Grundkurven werden zur Geometriemodellierung für jede Komponente definiert:

- Bauteilachse

- Spantarealkurve

- Querschnittsform 


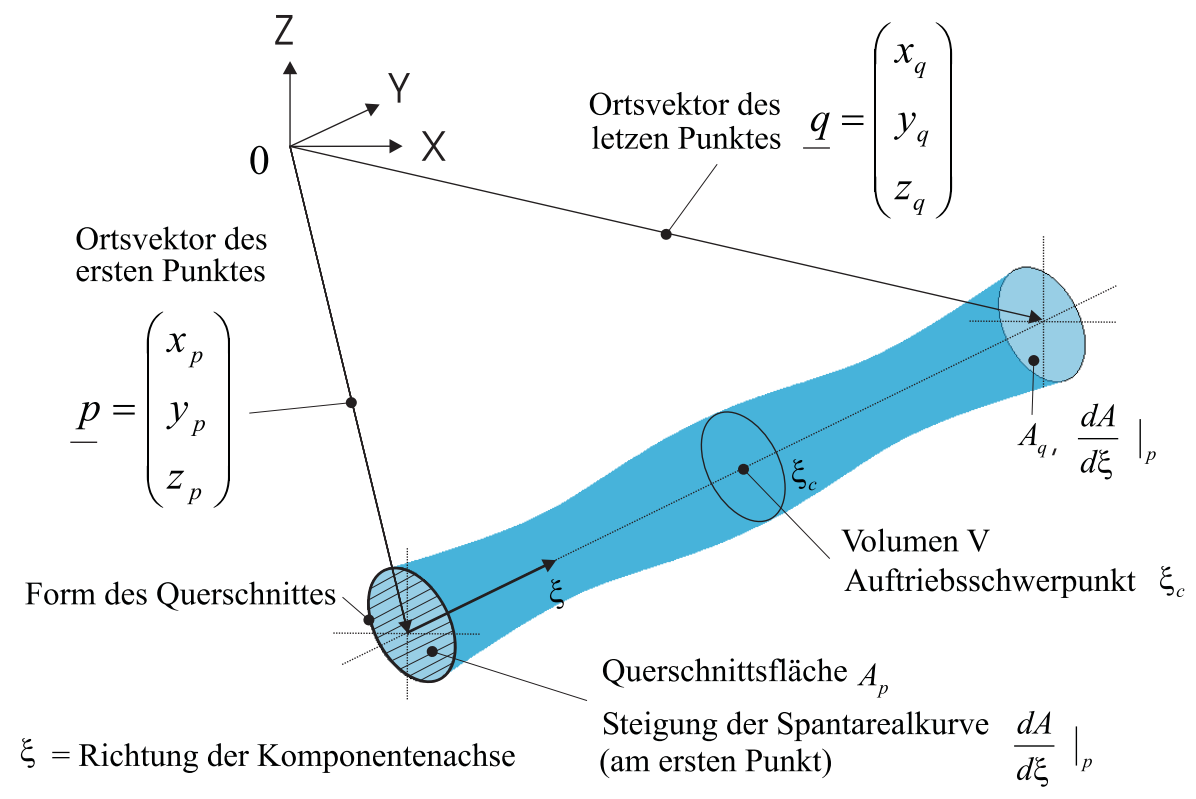

Abbildung 5.1: Formparameter

In der hydrodynamischen Formoptimierung ist besonders die Verdrängungsverteilung von Bedeutung, da sie einen wesentlichen Einfluss auf die Seegangskräfte sowie hydrodynamische Massen hat. Jede Strukturkomponente wird deshalb durch Parameter definiert, die eine Festlegung und Variation der Verdrängungsverteilung gestatten (Abb. 5.1). Die beiden Ortsvektoren $\underline{p}=\left(x_{p}, y_{p}, z_{p}\right)^{T}$ und $\underline{q}=\left(x_{q}, y_{q}, z_{q}\right)^{T}$ markieren Beginn und Ende der Bauteilachse und legen so die Lage der Komponente im Raum fest. Eine dimensionslose Koordinate $\underline{\xi}(0 \leq \xi \leq 1)$ beschreibt Punkte entlang der Achse. Aus Volumen $V$, der Lage des Verdrängungsschwerpunktes $\xi_{c}$ auf der $\xi$ Achse, den Querschnittsflächen $A_{p}$ und $A_{q}$ sowie deren Ableitungen bezüglich $\xi$ an den Endpunkten $d A / d \xi_{p}$ und $d A / d \xi_{q}$ wird die Verdrängungsverteilung in Form einer Spantarealkurve festgelegt (Abb. 5.2). Aus diesen Formparametern (Abb. 5.1) lässt sich eine Spantarealkurve z.B. mittels der Methode der Einflußpolynome (Taylor,1915; Weinblum,1953;Kwik, 1969;Nowacki und Reed,1974) generieren, wobei der Anwender auf vier unterschiedliche Parametersätze zurückgreifen kann:

1. $A_{p}, A_{q}, V$ und $\xi_{c}$

2. $A_{p}, A_{q}, V, \xi_{c}$ und $d A /\left.d \xi\right|_{p}$

3. $A_{p}, A_{q}, V, \xi_{c}$ und $d A /\left.d \xi\right|_{q}$

4. $A_{p}, A_{q}, V, \xi_{c}, d A /\left.d \xi\right|_{p}$ und $d A /\left.d \xi\right|_{q}$

Das von Nowacki und Reed benutzte Verfahren arbeitet zwar stabil, jedoch ist die Formenvielfalt begrenzt (Clauss und Birk, 1995b). Zur Verbesserung der Formgenerierung werden daher die Grundlinien als Non-Uniform-Rational-B-Spline (NURBS) gespeichert (Huang, 2000). Details über die mathematische Darstellung der Spantarealkurve mittels einer B-Spline-Kurve finden sich in Anhang A. 


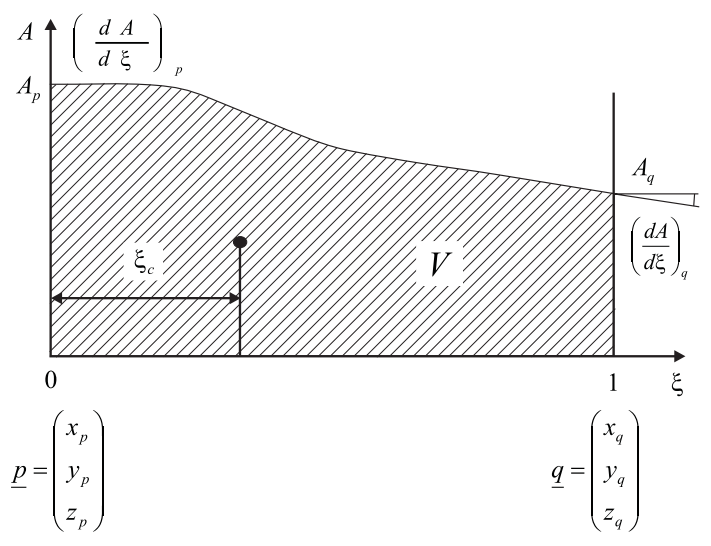

(a) Spantarealkurve und Komponentenachse

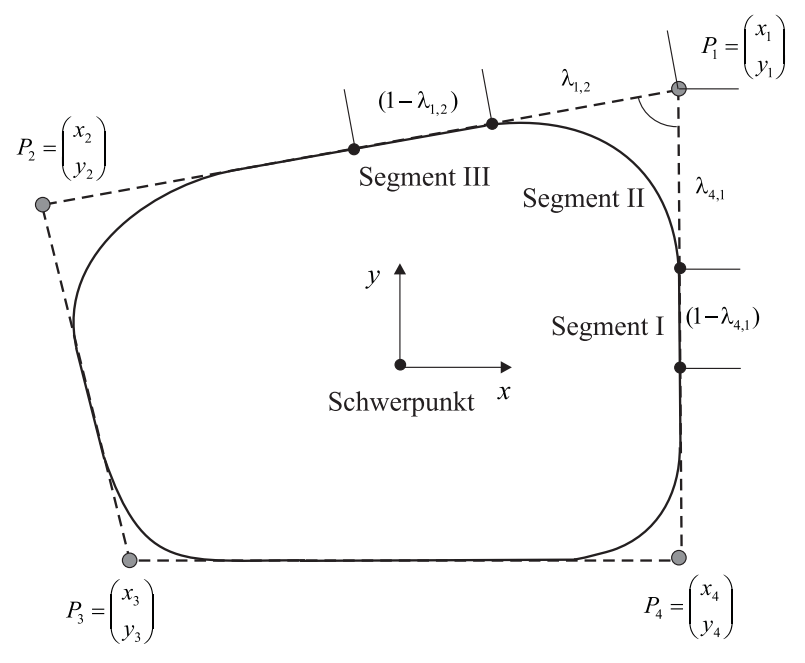

(b) Spantform

Abbildung 5.2: Grundkurven und Parameter der parametrischen Geometrieerzeugung einer Strukturkomponente

Die dritte Basiskurve definiert die Spantform der Komponente durch vier mehrsegmentige NURBS-Kurven. Vier Eckpunkte $\underline{P}_{1}$ bis $\underline{P}_{4}$ legen die Grundform des Querschnitts fest (Abb. 5.2(b)). Die Mittelpunkte der Viereckseiten dienen als Start- und Endpunkte der NURBS-Kurven, die aus zwei geraden Segmenten an den Enden und einem elliptischen Bogen in der Mitte (Segment II) bestehen. Mit den Rundungsparametern $\lambda_{i, j}$ können die Anteile von Geraden und Bogen so verändert werden, dass von Kreisen über Ellipsen, bis hin zu beliebigen Vierecken mit scharfen oder gerundeten Kanten die meisten üblichen Querschnittsformen meerestechnischer Konstruktionen beschrieben werden können. Die Formparameter für Achse, Spantarealkurve und Querschnitt müssen für jede Strukturkomponente definiert werden. Dabei können Parameter der einen Komponente von Parametern der anderen Komponente abhängen. Topologische Zusammenhänge, wie Schnittpunkte und -kurven zwischen Strukturkomponenten, können so einfach implementiert werden.

Bei hydrodynamisch kompakten Strukturen (siehe Abschnitt 5.2) wird zur Vergrößerung der Formenvielfalt ein erweitertes Formgenerierungsverfahren von Huang (2000) eingesetzt, das mit der Programmiersprache Python (van Rossum und Drake Jr., 2003) entwickelt wurde. Durch eine modifizierte Sweeping-Operation wird die Oberfläche der Komponente aus den Grundkurven erzeugt und als NURBS-Fläche gespeichert. Diese Formgenerierungstechnik ermöglicht eine Variation der Form des Querschnitts. Durch Änderungen der Formparameter kann die Querschnittsformen zwischen Kreis und Ellipse bis zum Viereck mit und ohne abgerundeten Ecken variieren. Abb. 5.3 zeigt den Prozess des Zusammenfügens einzelner Komponenten am Beispiel eines Halbtauchers. Nachdem alle Komponenten aus Formparametern generiert wurden (a), werden die Überlappungsbereiche aller Komponenten berechnet. Wo nötig -wie hier zwischen Säule und Ponton wird eine Komponente gekürzt und ein Ausschnitt erzeugt, um Platz für Übergangsflächen zu machen (b). Die Übergangsflächen werden aus den Rand- 
kurven automatisch erzeugt (c) und die Komponenten können zu einer geschlossenen Oberflächenbeschreibung zusammengefügt werden (d). Details über die Berechnung der Übergangsflächen sind bei Huang (2000) beschrieben.

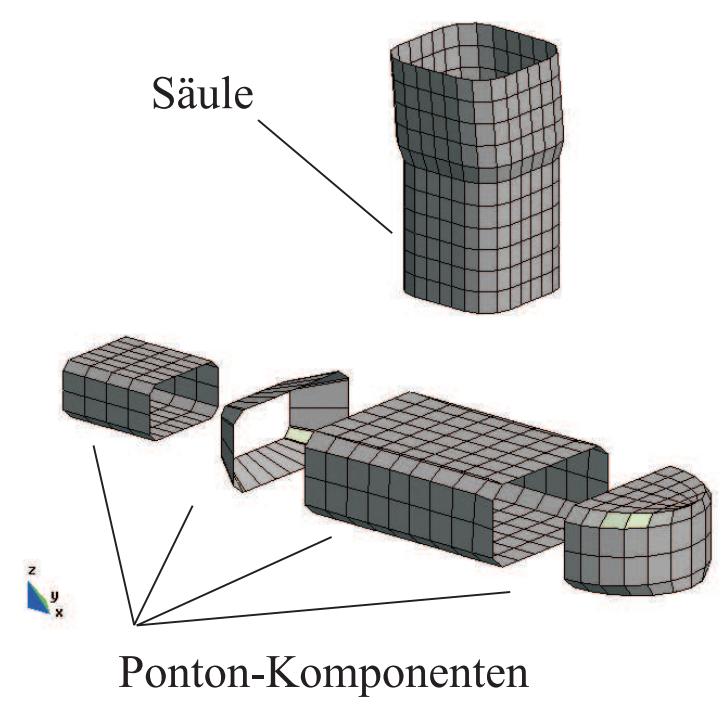

(a) Komponenten erzeugen

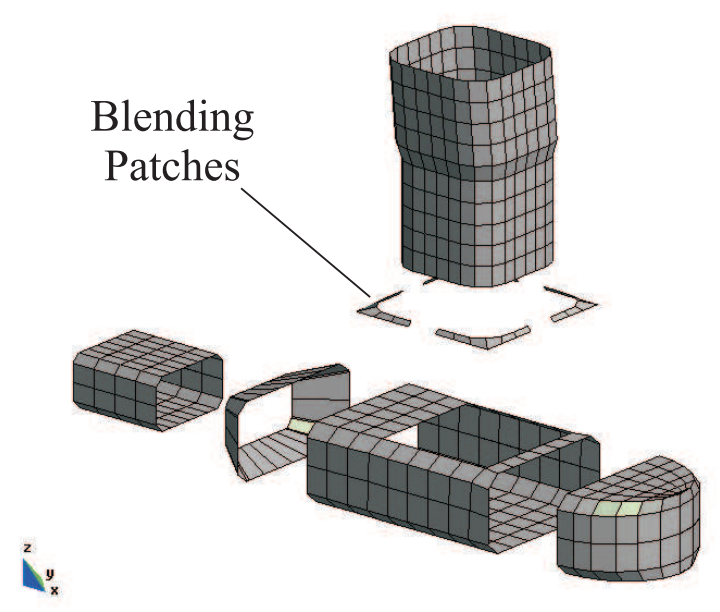

(c) Füllflächen erzeugen
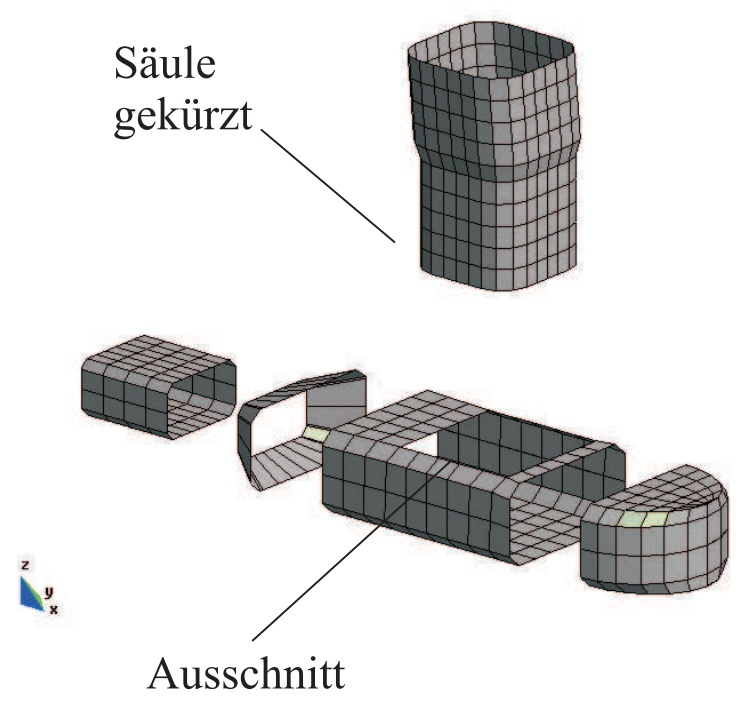

(b) ausschneiden und kürzen

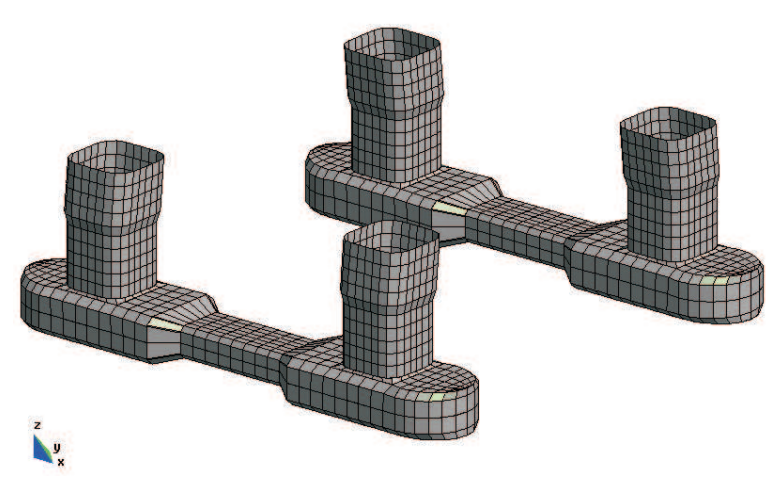

(d) zusammenfügen und spiegeln

Abbildung 5.3: Zusammenfügen mehrerer Komponenten mit Übergangsflächen (blending patches) am Beispiel eines hydrodynamisch kompakten Halbtauchers 
Bei hydrodynamisch transparenten Strukturen (siehe Abschnitt 5.2) ist keine Beschreibung der Komponentenoberfläche erforderlich, da die Strömungsgrößen Druck, Beschleunigungen usw. nicht auf der realen benetzten Oberfläche sondern näherungsweise auf der Bauteilachse bestimmt werden können. Insofern reichen Bauteilachsen und Spantarealkurven der Komponenten zur Beschreibung der Geometrie der Konstruktion aus. Die Querschnittsfläche für eine Strukturkomponente wird entweder kreisförmig oder rechteckig definiert. Zusätzlich müssen hydrodynamische Eigenschaften der Querschnittsform durch Koeffizienten vorgegeben werden.

\subsection{Hydrodynamische Analyse}

Meerestechnische Konstruktionen werden hinsichtlich ihrer Wechselwirkung mit dem Seegang in zwei Klassen aufgeteilt:

- Hydrodynamisch transparente Strukturen und

- hydrodynamisch kompakte Strukturen.

Hydrodynamisch transparente Strukturen verändern das Wellenfeld nicht, d.h. die Wellen passieren die Struktur ungestört. Dies trifft für Strukturen zu, deren charakteristischer Bauteildurchmesser $D$ kleiner als ein Fünftel der Wellenlänge $L_{w}$ ist $\left(D / L_{w}<0.2\right)$, wie z.B. Stahljackets und kleinere Halbtaucher. Da die Wellenlänge abhängig von der Wellenkreisfrequenz $\omega$ ist, können Strukturen in einem Seegangszustand transparent, in einem anderen kompakt sein.

Kräfte und Bewegungen hydrodynamisch transparenter Strukturen werden auf Basis der semi-empirischen Morison-Gleichung ermittelt. Diese, ursprünglich für feststehende, senkrechte Pfähle entwickelte Formel (Morison et al., 1950) wurde später auf beliebig orientierte Bauteile erweitert (siehe z.B. Clauss et al., 1992). Die vektorielle Kraft $d \underline{f}$ auf eine infinitesimal dicke Scheibe des Bauteils ist durch einen Trägheitskraftanteil $d \underline{f}_{m}$ und durch einen nichtlinearen Widerstandskraftanteil $d \underline{f}_{d}$ gegeben:

$$
d \underline{f}=d \underline{f}_{m}+d \underline{f}_{d}
$$

Die Strömungsgrößen werden näherungsweise an der Bauteilachse ermittelt, da - aufgrund der hydrodynamischen Transparenz der Struktur - eine Veränderung der Strömung vernachlässigt werden kann. Die Form des Bauteilquerschnitts wird durch den Trägheitsbeiwert $C_{m}$ und den zähigkeitsbedingten Widerstandbeiwert $C_{d}$ berücksichtigt. Um die Erregerkräfte sowie die hydrodynamischen Massen und Dämpfungen für die linearisierten Bewegungsgleichungen zu erhalten, wird die Morison-Kraft (5.1) in die (bekannten) seegangsabhängigen und (unbekannten) bewegungsabhängigen Anteile aufgeteilt. Die Integration von (5.1) über die getauchten Bauteillängen liefert die für die Gesamtstruktur gültigen Größen.

Für die hydrodynamische Formoptimierung hydrodynamisch transparenter Strukturen wurde das Programm TFC (Transfer Function Calculation) entwickelt, das die Übertragungsfunktionen für Kräfte und Bewegungen weitgehend analytisch und dadurch sehr viel schneller ermittelt, als mit einem 3D-Panelverfahren (Clauss et al., 
2002). Details über die Implementierung des TFC-Programmes sowie die Validierung mit Versuchdaten finden sich in Anhang B. Durch Linearisierung der zähigkeitsbedingten Widerstandskraft wird das lineare, komplexe Gleichungssystem (siehe Gl. B.105) gelöst, wobei die mit der unbekannten Körperbewegung gekoppelte Erregerkraft sowie die Dämpfungsmatrix mit dem Startvektor (Nullvektor) iterativ berechnet werden (siehe Abschnitt B.6). Die Konvergenz der Lösung ist von der Wellenkreisfrequenz abhängig. Im Bereich der Resonanz- und der Auslöschungsfrequenz, bei denen die Zähigkeitsdämpfung eine Rolle spielt, benötigt die Berechnung mehr Iterationsschritte. Im übrigen Frequenzbereich konvergiert die Lösung schnell.

Streng genommen ist das Konzept der Übertragungsfunktionen nur auf lineare Systeme anwendbar, d.h. Systeme, deren Antwort (Bewegung, Kraft) proportional zur Erregung (Welle) sind. Da die zähigkeitsbedingte Widerstandskraft von dem Quadrat der Wellenamplitude $\zeta_{a}^{2}$ abhängig ist, ist die Übertragungsfunktion nach der Linearisierung noch von der Wellenamplitude $\zeta_{a}$ abhängig (siehe Gl. B.38 und Gl. B.42). Antwort und Erregung sind demnach nicht proportional. Die hier mit dem TFC-programm berechneten Übertragungsfunktionen sind von der Wellenamplitude abhängig, die für die Berechnung der Dämpfungsmatrix und den zähigkeitsbedingten Anteil der Erregerkraft verwendet wurde. Ein Vergleich unterschiedlicher Übertragungsfunktionen (andere Freiheitsgrade, anderes System) kann deshalb nur bei gleicher Wellenamplitude vorgenommen werden. Die zähigkeitsbedingten Effekte sind jedoch auf den Bereich der Resonanz- und der Auslöschungsfrequenz begrenzt.

Der charakteristische Bauteildurchmesser hydrodynamisch kompakter Strukturen ist so groß, dass sie das Wellenfeld in ihrer Umgebung durch Diffraktion (Beugung) und Reflexion merklich verändern $\left(D / L_{w} \geq 0.2\right)$. Da bei kompakten Strukturen Zähigkeitskräfte vernachlässigbar klein gegenüber den Trägheitskräften sind, kann das Strömungsfeld auf potentialtheoretischer Basis berechnet werden (Newman, 1977; Clauss et al., 1992). Dazu muss die Laplacegleichung zusammen mit den entsprechenden Randbedingungen auf der Körperoberfläche, am Meeresboden, an der freien Flüssigkeitsoberfläche und im Unendlichen gelöst werden. Zuverlässige numerische Verfahren für diese Diffraktions-Radiations Probleme bei Offshore-Strukturen entstanden in den achtziger Jahren (Breit et al., 1985). Für die hydrodynamische Formoptimierung wird das lineare Diffraktions-Radiations Programm $W A$ MIT ${ }^{\circledR}{ }^{1}$ eingesetzt (WAMIT, 1994), das von Newman und seiner Arbeitsgruppe am Ocean Engineering Department des MIT entwickelt wurde (WAMIT, 1994). Details über die Grundlagen des 3D-Diffraktion-Radiation-Programmes WAMIT finden sich in Anhang C.

Die Bewegungen der Konstruktion in allen sechs Freiheitsgraden $\underline{s}$, gewinnt man durch Lösung der linearisierten Bewegungsgleichungen. Mit einem harmonischen Lösungsansatz für Bewegungen und Erregerkraft,

$$
\underline{s}=\underline{\widehat{s}} \cdot e^{-i \omega t} \text { und } \underline{F}_{e r r}=\underline{\widehat{F}}_{e r r} \cdot e^{-i \omega t}
$$

erhält man:

$$
\left[-\omega^{2}(\underline{\underline{M}}+\underline{\underline{A}})-i \omega \underline{\underline{B}}+\underline{\underline{C}}\right] \frac{\underline{s}}{\zeta_{a}}=\frac{\underline{F}_{e r r}}{\zeta_{a}} .
$$

\footnotetext{
${ }^{1}$ WAMIT: Wave Analysis Massachusetts Institute of Technology; WAMIT ist ein eingetragenes Warenzeichen von WAMIT Inc., Chestnut Hill MA, USA.
} 
$\underline{\widehat{F}}_{e r r}$ und $\underline{\widehat{s}}$ sind die Vektoren der so genannten komplexen Amplituden, d.h. sie beinhalten außer dem Betrag auch noch die Phaseninformation. Die Matrix der hydrodynamischen Massen $\underline{\underline{A}}$, die Matrix der hydrodynamischen Dämpfungen $\underline{B}$ und der Vektor der Erregerkräfte bzw. -momente $\underline{F}_{e r r}$ hängen von der Wellenkreisfrequenz $\omega$ ab. Durch die Linearisierung werden alle Größen (bis auf zähigkeitsbedingte Dämpfungen und Widerstandkräfte) proportional zur Wellenamplitude $\zeta_{A}$ und man erhält die unbekannten Bewegungen in Form von komplexen Übertragungsfunktionen

$$
H_{i}(\omega)=\frac{\widehat{s}_{i}}{\zeta_{a}}=\frac{s_{a}}{\zeta_{a}} \cdot e^{i \varepsilon}
$$

Sie geben das Verhältnis von Systemantwort (Bewegung) und Erregung (Seegang) in harmonischen Wellen der Frequenz $\omega$ wieder. $s_{a}$ ist die reelle Amplitude und $\varepsilon$ die Phasenverschiebung in Bezug auf die Wellenerhebung.

Da bei der numerischen Berechnung nur eine endliche Zahl von Wellenfrequenzen berücksichtigt werden kann, müssen neben den bekannten Diskretisierungsfehlern der Geometrie auch die Diskretisierungsfehler im Frequenzbereich berücksichtigt werden. Feine Details der Übertragungsfunktonen, wie z.B. Resonanzspitzen, könnten leicht übersehen werden, oder durch zufälliges Auftreten im Ergebnis den Optimierungsablauf verfälschen.

\subsection{Zielfunktionen}

Für den Entwurf des zu optimierenden Systems sind relevante Kriterien erforderlich, um einen schlechten von einem guten Entwurf zu unterscheiden. Abb. 5.4 zeigt schematisch den Ablauf der Bestimmung der Zielfunktion. Das mathematische Modell der Zielfunktion stellt einen Zusammenhang zwischen den gewählten freien Variablen $\mathbf{x}=\left(x_{1}, x_{2}, \cdots, x_{n}\right)^{T}$ sowie zusätzlichen, unveränderlichen Parametern $\mathbf{p}=$ $\left(p_{1}, p_{2}, \cdots, p_{m}\right)^{T}$ und dem Gütekriterium bzw. der Zielfunktion $f(\mathbf{x}, \mathbf{p})$ her.

In wenigen Fällen wird dieses Modell aus einer expliziten Gleichung für die zu minimierende Funktion $f$ bestehen. Je nach Komplexität und Umfang der Zielfunktion kann die Implementierung eines Modells mehrere komplexe Programmsysteme umfassen. Verschiedene Aspekte der technischen und ökonomischen Disziplinen können darin

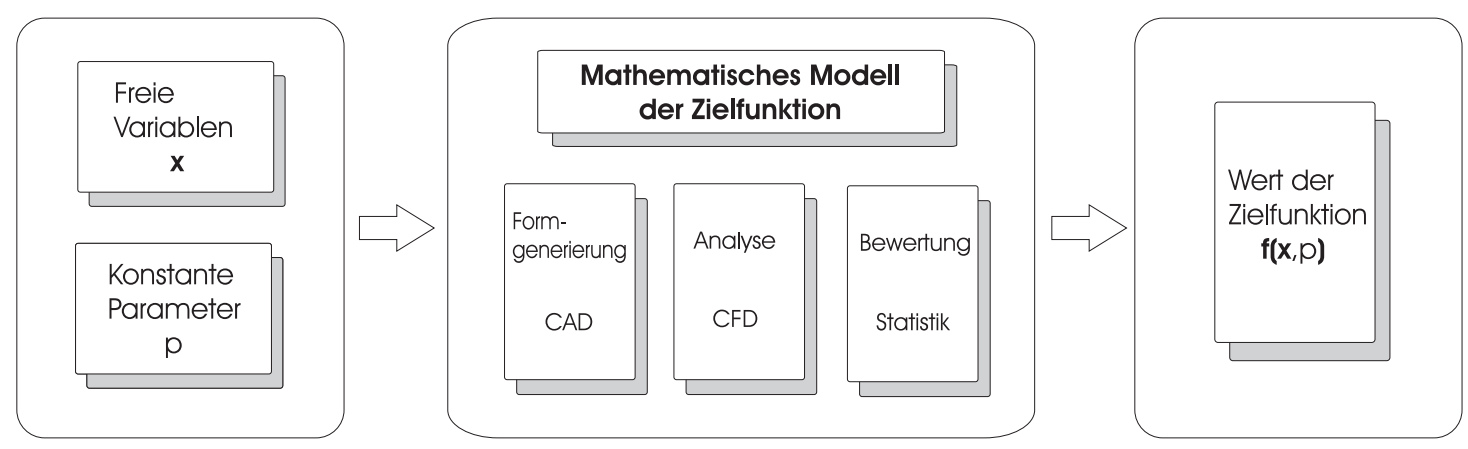

Abbildung 5.4: Mathematisches Modell der Zielfunktion 
behandelt werden. In der hier beschriebenen hydrodynamischen Formoptimierung soll der Entwurf bezüglich der Seegangseigenschaften der Systeme bewertet werden. Das bedeutet, es sollen Geometrien entwickelt werden, die sich durch geringe Belastungen und Bewegungen auszeichnen.

\subsubsection{Spektralanalyse}

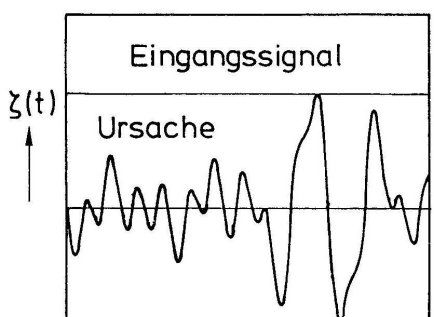

Wellenerregung
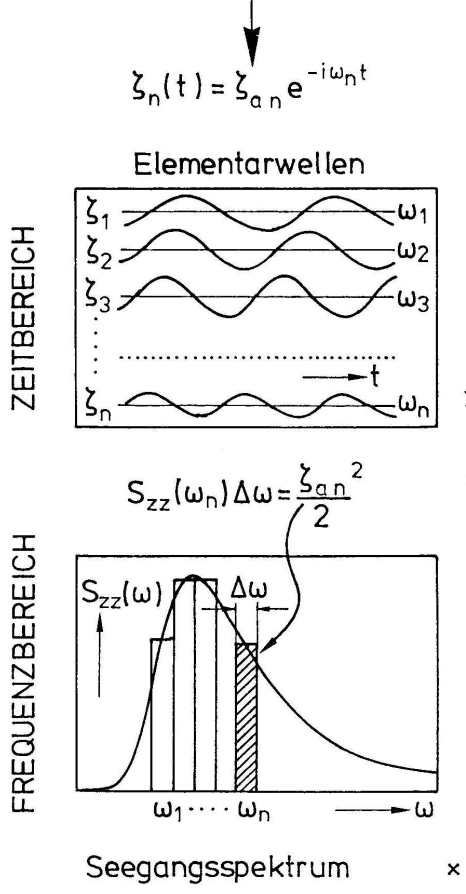

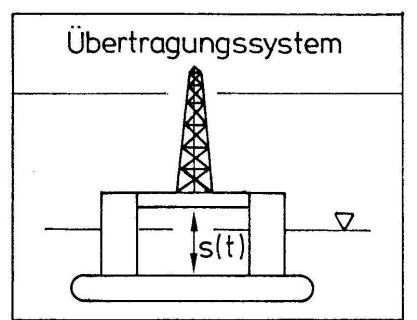

Meerestechnische Konstruktion Übertragungsfunktion

$H(\omega)=\frac{S_{a n}}{\zeta_{a n}} e^{i \varepsilon_{n}}$
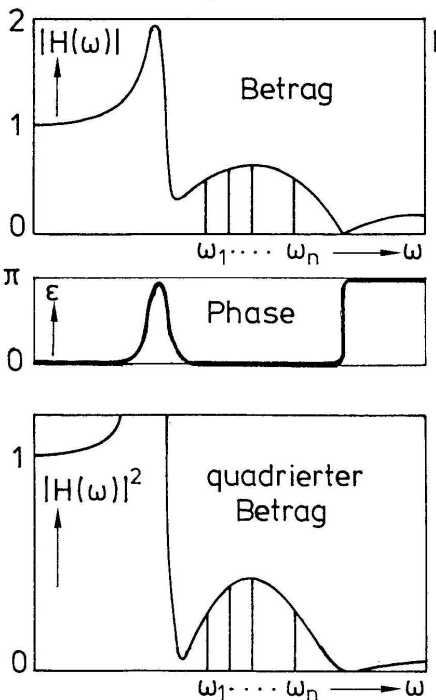
Quadrat des Betrages der
Übertraaunasfunktion

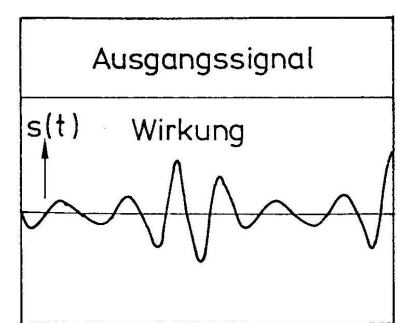

Kräfte und Bewegungen
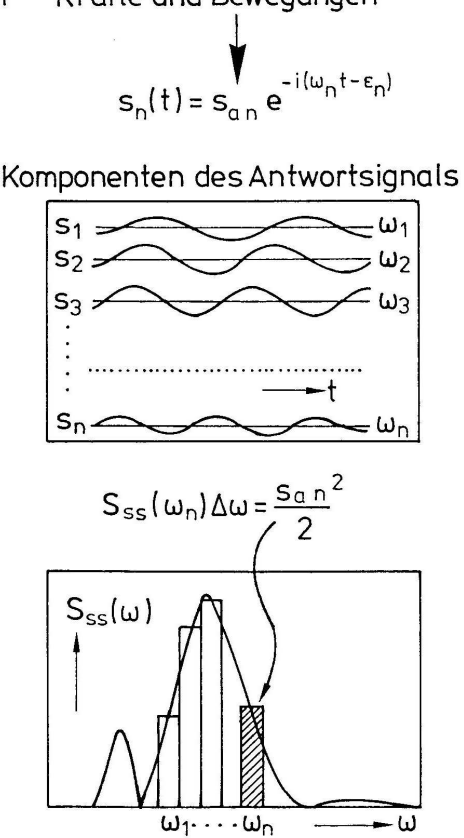

= Spektrum des Antwortsignals

Abbildung 5.5: Übertragungsfunktion einer meerestechnischen Konstruktion

(Clauss et al., 1992)

In der Schiffsdynamik fängt die Anwendung der Spektralanalyse mit der fundamentalen Publikation von St. Denis und Pierson (1953) an. Auf See kommen harmonische Wellen praktisch nicht vor. Lediglich die von weit entfernten Stürmen erzeugte Dünung (Swell) kann mit harmonischen Wellen verglichen werden. Die Erfahrung hat jedoch gezeigt, dass durch lineare Superposition vieler harmonischer Wellen unterschiedlicher Frequenz, Amplitude und Phase irregulärer Seegang sehr gut approximiert werden kann, wenn keine extreme Wellenamplitude auftritt. Jede harmonische Komponente $n$ 
erbringt einen Beitrag zur Gesamtenergie des Seegangs, der dem Quadrat der entsprechenden Wellenamplitude $\zeta_{a n}$ proportional ist:

$$
S_{\zeta}\left(\omega_{n}\right) d \omega=\frac{1}{2} \zeta_{a n}^{2}
$$

Das Energiedichtespektrum $S_{\zeta}$ stellt die Verteilung der Energie in Abhängigkeit von der Wellenfrequenz $\omega$ dar (Abb. 5.5).

Der Begriff der Energiedichtespektren ist eng mit der Wahrscheinlichkeitstheorie verknüpft. So kann Seegang jeweils für die Dauer von zwei bis drei Stunden in guter Näherung als ein stationärer Zufallsprozess betrachtet werden, bei dem die Wasserspiegelauslenkung einer Gaußschen Wahrscheinlichkeitsdichteverteilung folgt. Die Fläche $m_{0 \zeta}$ unter dem Energiedichtespektrum entspricht der Varianz $\sigma^{2}$ des Zufallsprozesses. Für Entwurfszwecke verwendet man in Ermangelung von in-situ gemessenen Spektren analytische, an Messungen angepasste Energiedichtespektren. Im Rahmen dieser Arbeit werden sogenannte Pierson-Moskowitz Spektren eingesetzt, die von den Parametern signifikante Wellenhöhe $H_{s}$ und Aufwärtsnullstellenperiode (zero-up-crossing period) $T_{0}$ abhängen:

$$
S_{\zeta}=4 \pi^{3} \frac{H_{s}^{2}}{T_{0}^{4} \omega^{5}} \exp \left(-\frac{16 \pi^{3}}{T_{0}^{4} \omega^{4}}\right)
$$

Für allgemeine Zufallsprozess fanden Cartwright und Longuet-Higgins (1956) eine Formel für die Verteilung der Extremwerte in Abhängigkeit vom Bandbreitenparameter $\varepsilon$ (siehe auch Kinsman (1965)). Ein sehr breitbandiger Prozess zeigt kein ausgeprägtes Maximum im Frequenzbereich. Es gibt also mehr Maximalwerte als Aufwärtsnullstellen. Als Grenzfall ist vorstellbar, dass die Aufwärtsnullstellenperiode extrem groß wird und sich dadurch $\varepsilon=1$ ergibt. Im Gegensatz dazu treten beim schmalbandigen Prozess nur positive Maxima und negative Minima auf. Seegang mit $\varepsilon<0.8$ ist leidlich mit einer Rayleighverteilung der Extremwerte zu beschreiben. Daraus lässt sich dann nach Longuet-Higgins (1952) zeigen, dass auch die Wellenhöhen der Rayleighverteilung unterliegen. Unter dieser Voraussetzung ergibt sich, dass die signifikante Wellenhöhe $H_{s}$ proportional zur Wurzel aus der Varianz ist

$$
H_{s}=4 \sqrt{m_{0 \zeta}} .
$$

Die Varianz bzw. das nullte Moment $m_{0 \zeta}$ des Energiedichtespektrums erhält man durch einfache Integration über die Wellenkreisfrequenz $\omega$

$$
m_{0}=\int_{0}^{\infty} S_{\zeta}(\omega) d \omega
$$

Für die mittlere Zero-up-crossing Periode $T_{0}$ gilt die Beziehung

$$
T_{0}=2 \pi \sqrt{\frac{m_{0}}{m_{2}}}
$$

mit dem zweiten Moment des Energiedichtespektrums

$$
m_{2}=\int_{0}^{\infty} \omega^{2} S_{\zeta}(\omega) d \omega
$$


Die Schmalbandigkeit des Seegangs wird meist ohne weitere Prüfung als gegeben vorausgesetzt.

Auch die Strukturantwort im irregulären Seegang kann unter der Annahme des linearen Verhaltens als Überlagerung unendlich vieler harmonischer Elementarantworten dargestellt werden. Entsprechend dem Energiedichtespektrum des Seegangs $S_{\zeta}(\omega)$ kann auch der Strukturantwort ein Spektrum zugeordnet werden. Den Zusammenhang zwischen Seegangs- und Antwortspektrum liefert die Übertragungsfunktion $H(\omega)$ :

$$
S_{s s}(\omega)=|H(\omega)|^{2} \cdot S_{\zeta}(\omega) .
$$

In Analogie zu (5.7) folgt aus dem Antwortspektrum die signifikante Doppelamplitude der Strukturantwort $\left(2 s_{a}\right)_{s}$ :

$$
\left(2 s_{a}\right)_{s}=4 \sqrt{\int_{0}^{\infty} S_{s s}(\omega) d \omega} .
$$

Die signifikante Doppelamplitude der untersuchten Kraft oder Bewegung charakterisiert das Strukturverhalten in einem vorgegebenen Seegangszustand durch eine skalare Größe. Sie ist deshalb besonders gut als Zielfunktion für die hydrodynamische Formoptimierung geeignet.

\subsubsection{Bewertung der Ausfallzeit}

Um die Seegangseigenschaften einer Struktur in Bezug auf ihre gesamte Einsatzdauer an einer Lokation zu optimieren, muss die statistische Verteilung $r_{i j}$ der einzelnen, kurzzeitigen Seegangszustände $\left(H_{s i}, T_{0 j}\right)$ zur Bewertung herangezogen werden (Abb. 5.6). Die Seegangsstatistiken werden summarisch für ein Jahr, oder auch nach Seegangsrichtungen bzw. Jahreszeiten geordnet, angegeben. Entsprechende Aufzeichnungen findet man z. B. bei Hogben und Lumb (1967). In manchen Fällen kann, wie in Abb. 5.6 rechts gezeigt, die diskrete Häufigkeitsverteilung durch spezielle nichtlineare Regressionsansätze als kontinuierliche, zweidimensionale Wahrscheinlichkeitsdichteverteilungen $p\left(H_{s}, T_{0}\right)$ in analytischer Form dargestellt werden (Mathisen und Bitner-Gregersen, 1990).

Dem Offshore-Betrieb werden durch die Bewegungen der Plattform Grenzen gesetzt. So sind z.B. für das Setzen eines Blow-Out-Preventers (BOP) Tauchbewegungen bis maximal 1.0m zulässig (Clauss et al., 1992). Überschreiten die signifikanten Bewegungen der Plattform das zulässige vorgegebene Limit $\left(2 s_{a}\right)_{s, z u l}$, müssen die Arbeiten mit erheblichen Ausfallkosten eingestellt werden. Hydrodynamische Formoptimierung ermöglicht, die seegangsbedingten Ausfallzeiten (Downtime) zu reduzieren und die Einsatzmöglichkeiten einer neuen Konstruktion zu verbessern.

Systematische Berechnungen nach (5.11) und (5.12) für verschiedene mittlere Zeroup-crossing Perioden $T_{0}$ ergeben eine als signifikante Übertragungsfunktion bezeichnete Funktion, die nur noch von $T_{0}$ abhängig ist:

$$
\frac{\left(2 s_{a}\right)_{s}}{H_{s}}\left(T_{0}\right)=4 \cdot \frac{\sqrt{\int_{0}^{\infty} S_{s s}(\omega) d \omega}}{H_{s}}
$$




\begin{tabular}{|c|c|c|c|c|c|c|c|c|c|c|c|c|}
\hline \multirow{3}{*}{$\begin{array}{c}\text { Sign.wave } \\
\text { height } \\
H_{s}[m]\end{array}$} & \multicolumn{12}{|c|}{ Zero-up-crossing period } \\
\hline & 0 & 4 & 5 & 6 & 7 & 8 & 9 & 10 & 11 & 12 & 13 & 14 \\
\hline & 4 & 5 & 6 & 7 & 8 & 9 & 10 & 11 & & 13 & 14 & 15 \\
\hline $11.5-12.0$ & 0 & 0 & 0 & 0 & 0 & 0 & 0 & 1 & 0 & 0 & 0 & 0 \\
\hline $11.0-11.5$ & 0 & 0 & 0 & 0 & 0 & 0 & 0 & 1 & 0 & 0 & 0 & 0 \\
\hline $10.5-11.0$ & \multicolumn{3}{|c|}{ zulässige sign. } & 0 & 0 & 0 & 0 & 2 & 0 & 0 & 0 & 0 \\
\hline $10.0-10.5$ & \multirow{2}{*}{\multicolumn{3}{|c|}{$\begin{array}{l}\text { zulassige sign. } \\
\text { Wellenhöhe }\end{array}$}} & 0 & 0 & 0 & 0 & 1 & 0 & 0 & 0 & 0 \\
\hline $9.5-10.0$ & \multirow{2}{*}{\multicolumn{3}{|c|}{$\mathrm{H}_{s, z u l}$}} & 0 & 0 & 0 & 2 & 3 & & 0 & 0 & 0 \\
\hline $9.0-9.5$ & & & & 0 & 0 & 0 & 10 & 4 & 1 & 0 & 0 & 0 \\
\hline $8.5-9.0$ & 0 & 0 & 0 & 0 & 0 & 0 & 13 & 5 & \multirow{4}{*}{\multicolumn{4}{|c|}{$\begin{array}{l}\text { unzulässige } \\
\text { Seegangs- } \\
\text { zustände }\end{array}$}} \\
\hline $8.0-8.5$ & 0 & 0 & 0 & 0 & 0 & 6 & 22 & 3 & & & & \\
\hline $7.5-8.0$ & 0 & 0 & 0 & 0 & 0 & 19 & 27 & 5 & & & & \\
\hline $7.0-7.5$ & 0 & 0 & 0 & 0 & 3 & 30 & 14 & 1 & & & & 0 \\
\hline $6.5-7.0$ & 0 & 0 & 0 & 0 & 8 & 61 & 16 & 1 & 0 & 0 & 0 & 0 \\
\hline $6.0-$ & 0 & 0 & 0 & 0 & 31 & 91 & 7 & 3 & 0 & 0 & 0 & 0 \\
\hline $5.5-6.0$ & & 0 & 0 & 117 & 91 & 14 & 1 & & 0 & 0 & 0 \\
\hline $5.0-5.5$ & \multirow{2}{*}{\multicolumn{2}{|c|}{ Seegangs- }} & 0 & 7 & 194 & 75 & 17 & 0 & 1 & 0 & 0 & 0 \\
\hline $4.5-5.0$ & & & 0 & 79 & 256 & 86 & 16 & 1 & & 0 & 0 & 0 \\
\hline $4.0-4.5$ & & stände & 1 & 235 & 271 & 75 & 28 & 2 & 0 & 0 & 0 & 0 \\
\hline $3.5-4.0$ & 0 & 0 & 33 & 407 & 263 & 101 & 9 & 0 & 0 & 0 & 0 & 0 \\
\hline $3.0-3.5$ & 0 & 0 & 187 & 514 & 293 & 78 & 16 & 0 & & 0 & 0 & 1 \\
\hline & 0 & 16 & & 642 & 304 & 97 & 15 & 2 & 0 & 0 & 0 & 0 \\
\hline $2.0-2$ & 0 & 310 & 1010 & 744 & 283 & 72 & 18 & 3 & 0 & 0 & 0 & 0 \\
\hline $1.5-$ & 83 & 1151 & 1106 & 607 & 198 & 39 & $\begin{array}{r}1 \\
7\end{array}$ & 0 & 0 & 0 & 0 & 0 \\
\hline $1.0-1.5$ & 725 & 1600 & 873 & 365 & 70 & 7 & & 0 & 0 & 0 & 0 & 0 \\
\hline $0.5-$ & 729 & 755 & 328 & 98 & 19 & 3 & 0 & $\overline{0}$ & & \begin{tabular}{|l}
0 \\
\end{tabular} & 0 & 0 \\
\hline $0.0-0.5$ & 22 & 18 & 9 & 2 & 0 & 0 & 0 & 0 & & 0 & 0 & 0 \\
\hline
\end{tabular}

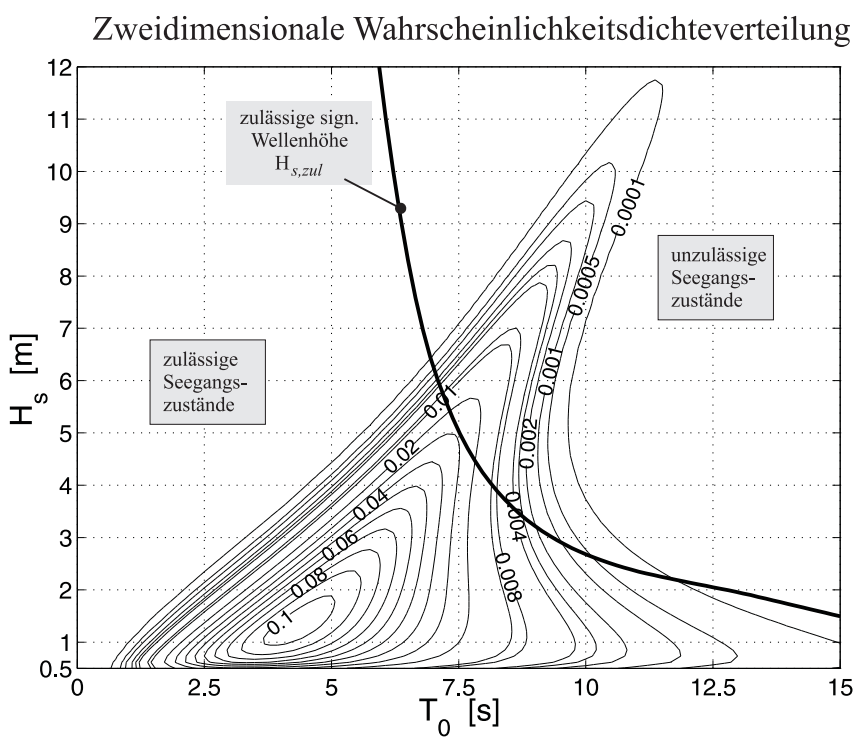

Abbildung 5.6: Absolute Häufigkeit und daraus abgeleitete zweidimensionale Wahrscheinlichkeitsdichteverteilung für ein Jahr (Seegebiet Haltenbanken vor der norwegischen Küste, Mathisen und Bitner-Gregersen, 1990). Beispiel für Grenzkurven der für den Betrieb zulässigen signifikanten Wellenhöhen

Hierbei wurde ausgenutzt, dass das Energiedichtespektrum des Seegangs proportional zum Quadrat der signifikanten Wellenhöhe (siehe Gl. 5.6) und damit die signifikante Antwort proportional zur signifikanten Wellenhöhe $H_{s}$ selbst ist. Mit der signifikanten Übertragungsfunktion (5.13) und der maximal zulässigen signifikanten Bewegungsamplitude $\left(2 s_{a}\right)_{s, z u l}$, kann die zulässige signifikante Wellenhöhe $H_{s, z u l}$ in Abhängigkeit der Zero-up-crossing Periode berechnet werden (siehe z.B. Price und Bishop (1974)).

$$
H_{s, z u l}\left(T_{0}\right)=\left(2 s_{a}\right)_{s, z u l}\left(\frac{\left(2 s_{a}\right)_{s}}{H_{s}}\right)^{-1} .
$$

Zeichnet man diese Funktion in die Seegangsstatistik ein, können die Seegangszustände $\left(H_{s i}, T_{0 j}\right)$ in zulässige und unzulässige Bereiche aufgeteilt werden (Abb. 5.6).

Die zu erwartende Ausfallzeit $P_{d}$ ergibt sich aus dem Verhältnis der Anzahl der unzulässigen Seegangszustände mit $H_{s}>H_{s, z u l}$ und der Gesamtzahl aller Beobachtungen $r=\sum_{i} \sum_{j} r_{i j}$ :

$$
\begin{aligned}
P_{d} & =\frac{\sum_{k} \sum_{j} r_{k j}}{r} \\
\text { wobei } k & \in\left\{i: H_{s i}>H_{s, z u l}\right\}
\end{aligned}
$$

Liegt die statistische Verteilung der Seegangszustände in Form von diskreten Tabellen vor, muss der Grenzwert für die zulässige signifikante Wellenhöhe auf die Klasseneinteilung der Tabelle gerundet werden (Stufenkurve in Abb. 5.6). Dies hat den Nachteil, dass die Zielfunktion unempfindlich gegen kleine Geometrieänderungen wird und für zwei leicht unterschiedliche Entwürfe den gleichen Zielfunktionswert ergeben können. Um 
kleine Verbesserungtrends der Zielfunktion nicht zu verlieren, wird hier für die hydrodynamische Formoptimierung die kontinuierliche Wahrscheinlichkeitsdichteverteilung eingesetzt, die mit einigem Mehraufwand eine feinere Auflösung der zu erwartenden Ausfallzeit erlaubt. Anstatt der einfachen Summation von relativen Häufigkeiten wird nun die Wahrscheinlichkeitsdichtefunktion $p\left(H_{s}, T_{0}\right)$ numerisch integriert.

$$
P_{d}=\int_{T_{0}} \int_{H_{s}} p\left(H_{s}>H_{s, z u l}, T_{0}\right) d H_{s} d T_{0}
$$

Um der ungleichförmigen Verteilung der Seegangsrichtungen (mittlere Fortschrittsrichtung der Wellen) und den vom Welleneinfallswinkel abhängigen hydrodynamischen Eigenschaften Rechnung zu tragen, können die Berechnungen der Ausfallzeit nach (5.15) oder (5.16) für die nach Seegangsrichtungen aufgesplitteten Seegangsstatistiken durchgeführt werden. Mit den Wahrscheinlichkeiten $q_{\beta}$ für das Auftreten eines Seegangs mit mittlerer Seegangsrichtung $\beta$ wird als Zielfunktion eine mittlere Ausfallzeit $\bar{P}_{d}$ definiert.

$$
\bar{P}_{d}=\sum_{\beta} P_{d \beta} \cdot q_{\beta}
$$

Hierbei bezeichnet $P_{d \beta}$ den für die Seegangsrichtung $\beta$ ermittelten Erwartungswert der Ausfallzeit.

\subsubsection{Bewertung von Ermüdungsschäden}

Offshore Strukturen sind zyklischen Belastungen ausgesetzt, die sich zeitlich ständig ändern. Ermüdungsschäden treten bei jeder Last auf, wenn die Spannungsamplitude $\sigma_{a}$ die Ermüdungsgrenze $\sigma_{D}$ überschreitet. Die sogenannte Wöhlerkurve (im Englisch S-N Kurven) stellen den Zusammenhang zwischen dem Spannungsniveau $\sigma_{a}$ und der Zahl der insgesamt ertragbaren Lastzyklen $N$ dar (Fricke et al., 1997). Hierbei beschreibt $N$ die Zahl der Spannungszyklen, die auf dem Spannungsniveau $\sigma_{a}$ zur ersten Rißbildung oder zum Durchriss des Probestückes führen. Ist die Zahl der Lastzyklen groß, können $\mathrm{S}-\mathrm{N}$ Kurven wie folgt approximiert werden:

$$
N\left(\sigma_{a}\right)=N_{D}\left(\frac{\sigma_{a}}{\sigma_{D}}\right)^{-k}
$$

Wie in Abb. 5.7 schematisch dargestellt, können S-N Kurven in den meisten Fällen als gerade Linien auf logarithmischer Skala dargestellt werden. Die dauerfeste Spannung $\sigma_{D}$ (Ermüdungsgrenze) und die Zahl der Lastzyklen $N_{D}$ sind vom Material abhängig und werden durch Tests bei konstanten Spannungsamplituden experimentell ermittelt. Der Exponent $k$ definiert die Neigung der Kurve. Das Spannungsniveau, bei dem die Kurve flach wird, nennt man die Ermüdungsgrenze $\sigma_{D}$ (fatigue limit). Es wird hierbei angenommen, dass zyklische Lasten, deren Spannungsamplituden unterhalb der Ermüdungsgrenze liegen, keine strukturelle Schädigung erbringen und deshalb die Lebensdauer nicht beeinträchtigen. Bei Offshore Strukturen beeinflussen jedoch der Zufallscharakter der Wellen und die Korrosion des Meeres den Ermüdungsprozess. Das 


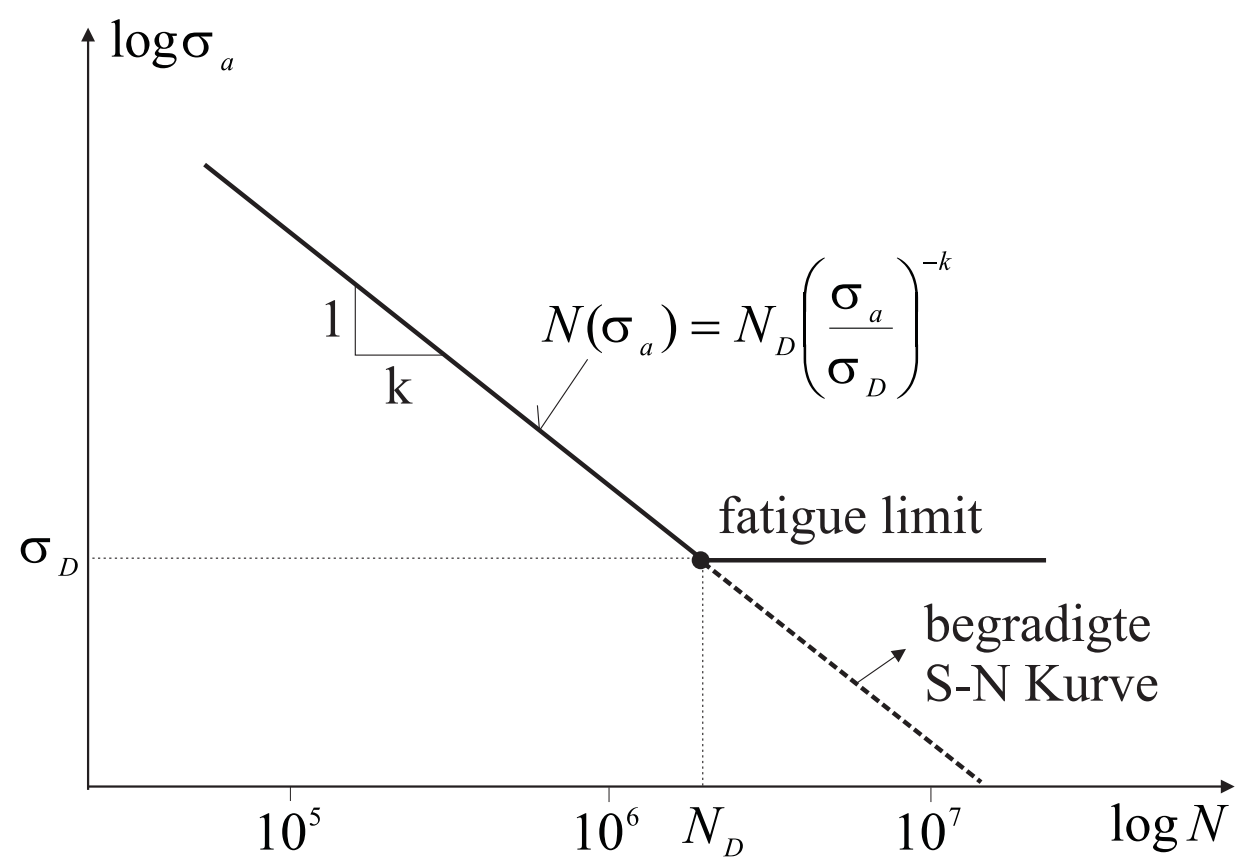

Abbildung 5.7: Prinzipielle Darstellung der S-N Kurve

führt in der Praxis dazu, dass aus Sicherheitsgründen die Ermüdungsgrenze vernachlässigt wird (Barltrop und Adams, 1991), so dass eine einfache begradigte S-N Kurve zur Berechnung der Ermüdungsschäden verwendet werden kann (siehe Abb. 5.7).

Die Vorhersage des Ermüdungsschadens wird erheblich schwieriger, wenn ein Probestück nicht mit konstanten Spannungsamplituden, sondern durch einen Zufallsprozess belastet wird. Zur Erfassung des Ermüdungsschadens infolge zufälliger Lasten wurde von Palmgren (1924) und Miner (1945) das Konzept der linearen Schadensakkumulationshypothese vorgeschlagen. Nach dieser Hypothese verursachen $n$ Lastzyklen des Spannungsniveaus $\sigma_{a}$ bei linearer Abhängigkeit die Schädigung

$$
D\left(\sigma_{a}\right)=\frac{n}{N\left(\sigma_{a}\right)}
$$

wobei $N\left(\sigma_{a}\right)$ nach (5.18) definiert wird. Es wird dabei angenommen, dass die Teilschädigung $D_{l}$ auf diesem Spannungsniveau $\sigma_{a}$ den entsprechenden Schädigungen anderer Spannungsniveaus hinzugefügt werden kann, um den Gesamtschaden $D$ zu erhalten:

$$
D=\sum_{l} D_{l}=\sum_{l} \frac{n_{l}}{N\left(\sigma_{a l}\right)}
$$

Wenn sich die Summation der Teilschädigungen $D_{l}$ über alle Spannungsniveaus dem Wert eins annähert, erhält man die hypothetische Lebensdauer der betrachteten Komponente einer Konstruktion:

$$
L \sim \frac{1}{D}
$$


Zur Ermüdungsanalyse meerestechnischer Konstruktionen sind folgende Informationen erforderlich (Brebbia und Walker, 1979):

- Seegangsstatistik

- Zusammenhang zwischen Seegang und Spannung für kritische Punkte (hot spots)

- Bestimmung der S-N Kurve

Zusätzlich ist der Spannungskonzentrationsfaktor (SCF) erforderlich, der vom Bauteil und von der Geometrie der geschweißten Verbindung abhängt. Um die zu erwartenden Ermüdungsschäden im irregulären Seegang zu ermitteln, wird zunächst nach (5.11) das Antwortspektrum der Spannungen $S_{\sigma i j}(\omega)$ für jedes Wellenspektrum $S_{\zeta i j}\left(H_{s}, T_{0}\right)$ berechnet:

$$
S_{\sigma i j}(\omega)=\left|H_{\sigma \zeta}(\omega)\right|^{2} \cdot S_{\zeta i j}(\omega) .
$$

Die mittlere Zero-Up-Crossing Periode $T_{0 \sigma i j}$ für die Spannungszyklen kann analog zu (5.9) mit dem ersten und zweiten Moment des Beanspruchungsspektrums berechnet werden

$$
T_{0 \sigma i j}=2 \pi \sqrt{\frac{m_{0 \sigma i j}}{m_{2 \sigma i j}}} .
$$

Damit lässt sich die Zahl der Spannungszyklen $n_{i j}$ in einem Seegang $S_{\zeta i j}$ ermitteln:

$$
n_{i j}=\frac{T_{i j}}{T_{\sigma i j}}
$$

wobei $T_{i j}$ die Dauer des betrachteten Seegang bezeichnet.

Mittels der Wahrscheinlichkeitsdichteverteilungen für die Maxima des Zufallsprozesses kann das zu erwartende Ereignis der Spannungsamplitude $\sigma_{a}$ berechnet werden (Crandall und Mark, 1973). Bei einem schmalbandigen Zufallsprozess ist die Wahrscheinlichkeitsdichteverteilung der Amplituden gleich der Rayleighverteilung

$$
p_{R}(\sigma)=\frac{\sigma}{m_{0 \sigma i j}} e^{\frac{-\sigma^{2}}{2 m_{0 \sigma i j}}}
$$

Damit erhält man die Zahl der Spannungszyklen für $\sigma \leq \sigma_{a}<\sigma+d \sigma$

$$
d n_{i j}=n_{i j} p_{R}(\sigma) d \sigma
$$

und die Teilschädigung $d D_{i j}$ nach (5.19)

$$
d D_{i j}=\frac{d n_{i j}}{N\left(\sigma_{a}\right)} .
$$

Setzt man die S-N Kurve (5.18) in (5.27) ein und integriert über die Spannungen, erhält man die Schädigung $D_{i j}$, die durch den Seegang $S_{\zeta i j}\left(H_{s i}, T_{o j}\right)$ verursacht werden kann:

$$
D_{i j}=\frac{n_{i j}}{N_{D} \sigma_{D}^{k} m_{0 \sigma i j}} \int_{0}^{\infty} \sigma^{k+1} e^{\frac{-\sigma^{2}}{2 m_{0 \sigma i j}}} d \sigma
$$


Da die Lösung für das uneigentliche Integral in (5.28) mit der Gammafunktion $\Gamma$ bekannt ist, lässt sich die Gleichung weiter entsprechend (5.29) umformen. Detaillierte Herleitungen finden sich in Birk (1998).

$$
D_{i j}=\Gamma\left(\frac{k}{2}+1\right) \frac{\left(2 m_{0 \sigma i j}\right)^{k / 2}}{N_{D} \sigma_{D}^{k}} \frac{T_{i j}}{T_{\sigma i j}}
$$

Schließlich erhält man die zu erwartende Gesamtschädigung $D$ nach Summation von (5.29) über alle $\left(H_{s i}, T_{o j}\right)$

$$
D=\sum_{j} \sum_{j} D_{i j}
$$

Die Ermüdungsanalyse kann nur eine grobe Schätzung des realen Systemverhaltens sein, da die Miner-Regel mit vielen Vereinfachungen verbunden ist. Nach der Palmgren-Miner-Hypothese wird z.B. die Reihenfolge der veränderlichen Lastzyklen nicht berücksichtigt. Es ist deshalb nicht zu erwarten, dass die Miner-Regel für jeden Werkstoff gleichermaßen zuverlässige Ermüdungsvorhersagen bzw. Lebensdauern liefert. Dennoch ist diese Ermüdungsanalyse als Kriterium zur vergleichenden Bewertung relativer Betriebsfestigkeit ausgewählter Konstruktionselemente von großer Bedeutung. 


\section{Kapitel 6}

\section{Globale Optimierungsstrategien im Vergleich}

Im folgenden werden anhand von zwei beispielhaften Anwendungen die im Kapitel 4 vorgestellten Optimierungsalgorithmen verglichen. Aufgrund der unterschiedlichen hydrodynamischen Analyse wird in hydrodynamisch transparente und kompakte Strukturen unterschieden. Bei hydrodynamisch transparenten Strukturen wird zusätzlich der Einfluss der Zähigkeit auf das Optimierungsergebnis untersucht.

\subsection{Optimierung hydrodynamisch transparenter Struk- turen}

Für die Exploration von Gas- und Öllagerstätten vor der Küste Norwegens (Seegebiet Haltenbanken) soll ein Halbtaucher (Verdrängung $\triangle=30000 \mathrm{~m}^{3}$ ) entwickelt werden. Die Möglichkeiten meerestechnischer Konstruktionen für das Arbeiten auf See sind durch die Vorgabe der zulässigen Bewegungen für den gewünschten Einsatz begrenzt. Bei einem Halbtaucher ist z.B. für das Setzen von Blow Out Preventer (BOP) und Riser eine maximale Amplitude der Tauchbewegung von $0.4 m-1.0 m$ zulässig (Clauss et al., 1992). Um die Einsatzmöglichkeiten zu verbessern, ist es zweckmäßig, die seegangsbedingte Ausfallzeit zu minimieren. Im Entwurf wird versucht, die statistisch zu erwartende Ausfallzeit nach (5.14) und (5.16) mit $\left(2 s_{3 a}\right)_{s, z u l}=1.0 \mathrm{~m} \mathrm{zu}$ minimieren.

Die Halbtaucher besitzen die heute übliche Standardkonfiguration aus vier Säulen und zwei Pontons (siehe Abb. 6.1 und Abb. 6.10). Im Rahmen dieser Arbeit werden zwei unterschiedliche Typen von Halbtauchern untersucht: Zunächst wird ein einfacher Halbtaucher entwickelt, der durch 4 freie Variablen definiert wird. Alle Komponenten haben hierbei einen kreiszylindrischen Querschnitt. Anschließend wird ein komplexer Halbtaucher mit neun freien Variablen optimiert, wobei die Form der Querschnittsfläche des Pontons rechteckig ist. 

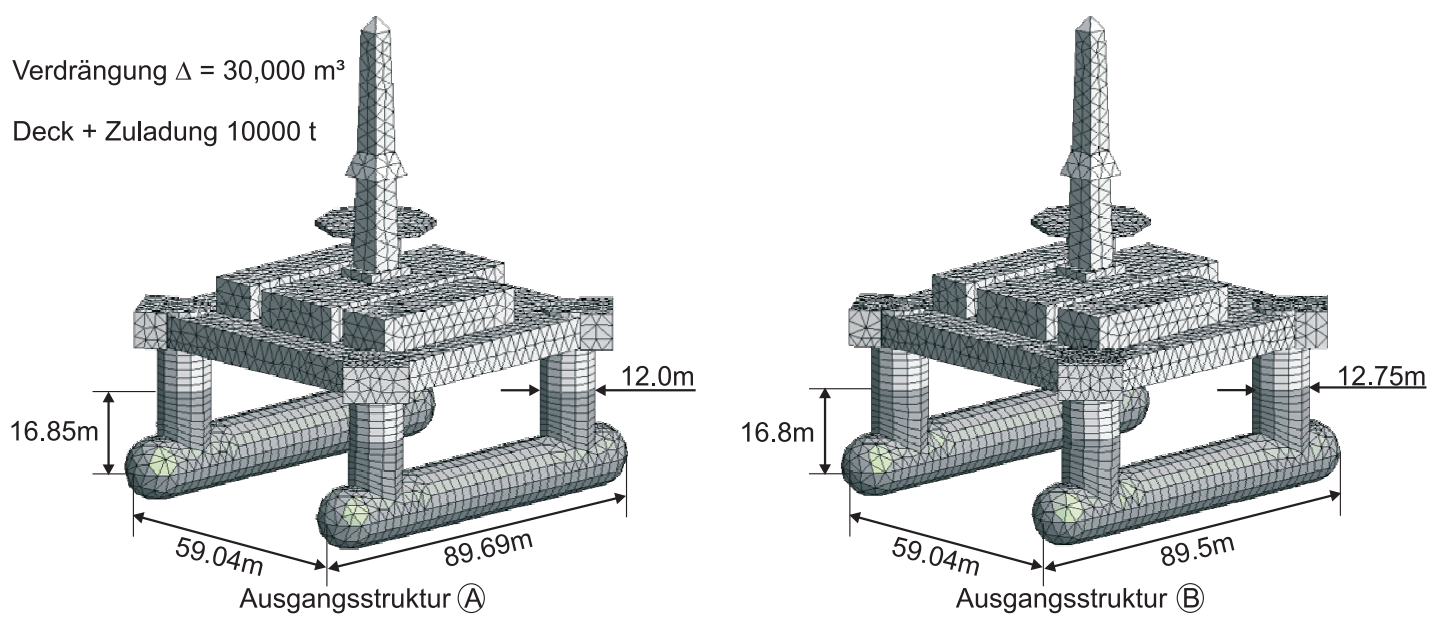

Abbildung 6.1: Geometrie der Ausgangsstrukturen (A) und (B)

\begin{tabular}{|c|c|c|c|}
\hline \multirow{2}{*}{\multicolumn{2}{|c|}{$\begin{array}{l}\text { freie } \\
\text { Variable }\end{array}$}} & \multicolumn{2}{|c|}{ Ausgangsstruktur } \\
\hline & & (A) & (B) \\
\hline$x_{1}$ & {$[-]$} & 0.85 & 0.83 \\
\hline$x_{2}$ & {$[m]$} & 12.0 & 12.75 \\
\hline$x_{3}$ & {$\left[\mathrm{~m}^{2}\right]$} & 149.8 & 145.8 \\
\hline$x_{4}$ & {$\left[m^{2}\right]$} & 149.8 & 145.8 \\
\hline
\end{tabular}

Tabelle 6.1: Definition der beiden Ausgangsstrukturen

\subsubsection{Halbtaucher mit 4 freien Variablen}

Abb 6.1 zeigt die gewählten Ausgangsstrukturen (A) und (B). Für die Optimierung wurden aus den Formparametern vier freie Variable ausgewählt (Tab. 6.1):

- Verhältnis des Pontonvolumens $\triangle_{p}$ zur Gesamtverdrängung $\triangle$ :

$$
x_{1}=\triangle_{p} / \triangle
$$

- Durchmesser der Säulen: $x_{2}=D_{s}$

- Querschnittsfläche des Pontons in der Mitte: $x_{3}=A_{p m}$

- Querschnittsfläche des Pontons vor der abschließenden Halbkugel: $x_{4}=A_{p e}$

Als Nebenbedingungen werden die minimale Querschnittsfläche der Pontons und die hydrostatische Anfangsstabilität der Konstruktion berücksichtigt:

- $A_{p, \min } \geq 50 m^{2}$

- $G M_{\text {min }} \geq 1 \mathrm{~m}$

- $\xi_{c}=0.5$ : die Lage des Verdrängungsschwerpunkts für ein Ponton 

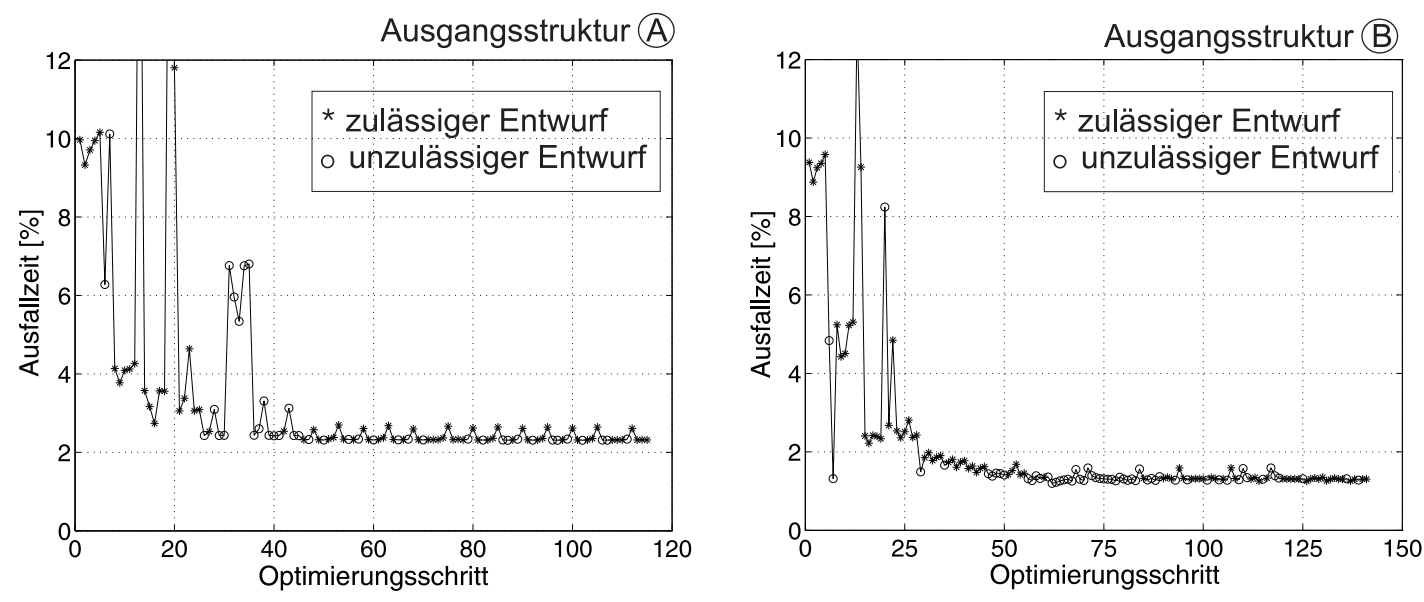

Abbildung 6.2: Optimierungsschritte und Änderung der Zielfunktion für SequentialQuadratic-Programming mit den Ausgangsstrukturen (A) und (B)

- $\Delta=30,000 m^{3}$

Aus Gründen der Längsfestigkeit darf die Querschnittsfläche der Pontons zwischen den Säulen den Wert $A_{p, \min }=50 \mathrm{~m}^{2}$ nicht unterschreiten. Die minimale metazentrische Höhe $\overline{G M}_{\text {min }}$ beträgt $1 \mathrm{~m}$. Um die Verschiebungen des Gewichtsschwerpunktes bei Variation der Geometrie zu berücksichtigen, wurde ein einfaches Massenmodell implementiert (siehe Clauss et al. (2002)). Die Gesamtverdrängung des Halbtauchers bleibt in allen Optimierungen konstant. Die untere und obere Schranke der definierten freien Variablen sind folgendermaßen festgelegt:

$$
\begin{aligned}
0.5 & \leq x_{1} \leq 0.9 \\
10 m & \leq x_{2} \leq 15 m \\
50 m^{2} & \leq x_{3} \leq 300 m^{2} \\
90 m^{2} & \leq x_{4} \leq 300 m^{2}
\end{aligned}
$$

Die Werte dieser Schranken wurden so ausgewählt, dass bei der Optimierung sowohl die Entartungen der Geometrie als auch die Verletzungen der Nebenbedingungen möglichst wenig auftreten. Die Optimierung soll also robust definiert sein.

Die Optimierungsaufgabe wurde nacheinander mit den Optimierungsstrategien Sequential Quadratic Programming (SQP), Genetischer Algorithmus (GA) und Adaptive Simulated Annealing (ASA) gelöst. Wichtige Parameter für die Steuerung der Optimierungsalgorithmen sind kurz zusammengefasst:

- Toleranz der Zielfunktionswertes: $1 e^{-6}$

- Toleranz der Nebenbedingungen: $1 e^{-6}$

- Toleranz der Schrittweite für freie Variable: $1 e^{-6}$

- Maximale Zahl der Funktionsauswertungen: 200 (SQP) 


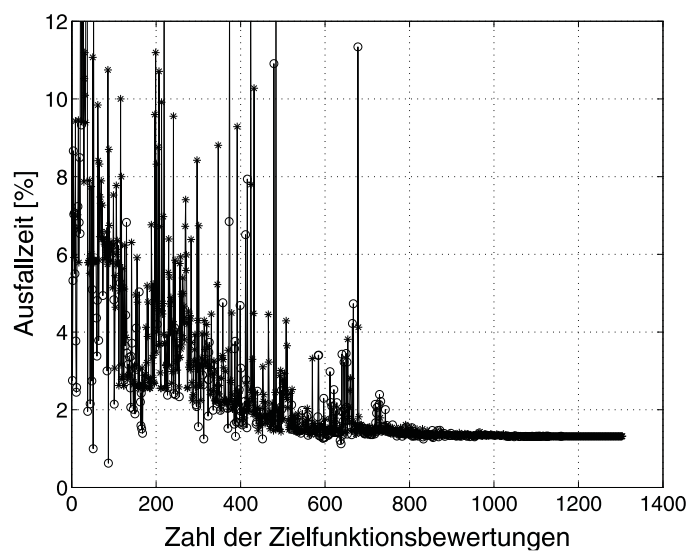

(a) Genetischer Algorithmus

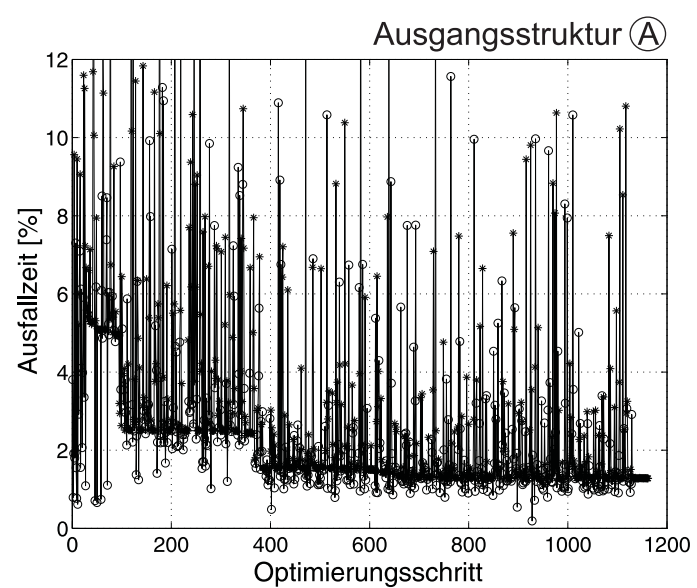

(b) Adaptive Simulated Annealing

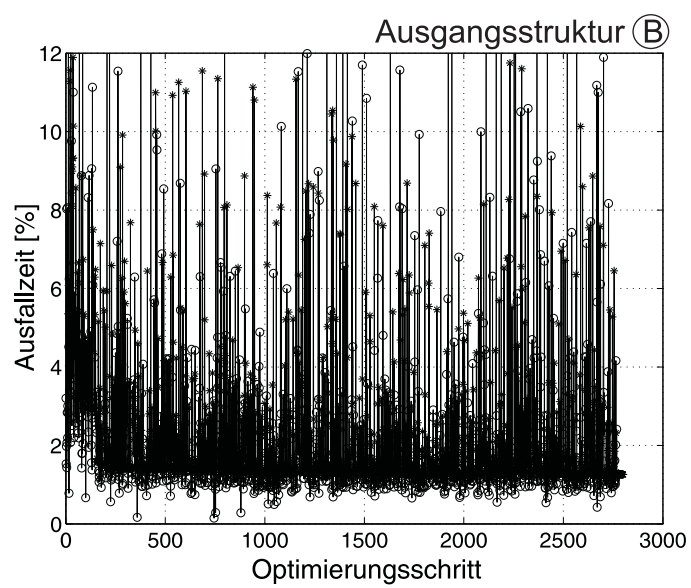

(c) Adaptive Simulated Annealing

Abbildung 6.3: Optimierungsschritte und Änderung der Zielfunktion (a) für Genetischen Algorithmus und (b),(c) für Adaptive Simulated Annealing Algorithmus

\begin{tabular}{|c|c|c|}
\hline \multicolumn{3}{|c|}{ Ausfallzeiten in \% [Zielfunktionsauswertungen } \\
\hline \multicolumn{3}{|c|}{ kein Zähigkeitseinfluss } \\
\hline $\begin{array}{l}\text { Frequenz- } \\
\text { schrittweite }\end{array}$ & \multicolumn{2}{|c|}{$\Delta \omega=0.025[\mathrm{rad} / \mathrm{s}]$} \\
\hline \multirow{2}{*}{$\begin{array}{l}\text { Ausgangs- } \\
\text { struktur }\end{array}$} & (A) & (B) \\
\hline & $9.97 \%$ & $9.38 \%$ \\
\hline Verfahren & \multicolumn{2}{|c|}{ Optimierte Strukturen } \\
\hline SQP & $2.31 \%[115]$ & $1.30 \%[141]$ \\
\hline ASA & $1.28 \%[1162]$ & $1.26 \%[2801]$ \\
\hline GA & \multicolumn{2}{|c|}{$1.31 \%[1304]$} \\
\hline
\end{tabular}

Tabelle 6.2: Vergleich der gefundenen Minima nach den Optimierungsstrategien 
- Maximale Zahl der Generationen: 100 (GA)

- Maximale Zahl der Akzeptanz: 100 (ASA)

In diesem Anwendungsbeispiel wird bei der Berechnung der Zielfunktion zunächst keine Zähigkeit berücksichtigt. Deshalb werden bei allen Entwürfen gleichermaßen Zähigkeitseinflüsse vernachlässigt. Streng genommen gilt dies nur für eindeutig hydrodynamisch kompakte Strukturen. Der Einfluss der Zähigkeit auf die Optimierung wird im Abschnitt 6.1.4 untersucht.

Abb. 6.2 zeigt den Verlauf der Optimierung unter Anwendung der lokalen SQP Strategie bei unterschiedlichen Ausgangsstrukturen. Entwürfe, die eine Nebenbedingung verletzen (unzulässiger Entwurf) sind mit einem Kreis (o) gekennzeichnet, zulässige Entwürfe mit einem Stern $(*)$. Die lokale SQP Strategie findet sehr schnell ein Minimum der Zielfunktion (mit der Ausgangsstruktur (A) nach 115 Schritten, mit (B) nach 141 Schritten). Damit ist die Anzahl der notwendigen Zielfunktionsauswertungen im Vergleich zu GA und ASA um eine Größenordnung kleiner (siehe Tab. 6.2). Bei der Ausgangsstruktur (A) wird die zu erwartende Ausfallzeit aufgrund großer Tauchbewegung deutlich von ca. $10 \%$ auf etwas mehr als $2 \%$ der Einsatzzeit reduziert. Mit der Ausgangsstruktur (B) trifft der SQP Algorithmus nach 141 Schritten-im Rahmen der numerischen Ungenauigkeiten- das Minimum mit einer Ausfallzeit von $P_{d}=1.30 \%$, das um 1\% besser als das mit der Ausgangsstruktur (A) erzielte Ergebnis ist (Abb.6.2). Bei technischen Optimierungsaufgaben unter Anwendung lokaler Suchstrategien ist es zu empfehlen, mit mehrfachen Ausgangsstrukturen die Optimierung durchzuführen. Jedoch sind die durch SQP erzielten Minima schlechter als die durch globale Optimierungsalgorithmen erzielten (siehe Tab. 6.2). Mit der Ausgangsstruktuktur (A) findet SQP nur ein lokales Minimum (siehe auch Abb. 6.5).

Bei globalen Optimierungsalgorithmen ist der Rechenaufwand deutlich größer als bei der lokalen Suchstrategie. Bei der Anwendung von GA werden als Steuerparameter für die Selektion der Individuen die sogenannte Ranking-Methode und für die $\mathrm{Mu}$ tation die Non-Uniform-Mutation verwendet (Houck et al., 1995b). Der Genetische Algorithmus braucht etwa 1300 Auswertungen der Zielfunktion, um ein Minimum von $P_{d}=1.31 \%$ zu finden. Um den Fortschritt des Genetischen Algorithmus zu dokumentieren, ist die Güte des besten Entwurfs einer Generation im Laufe der hydrodynamischen Optimierung in Abb. 6.4 aufgetragen. Jede Generation umfasst 10 verschiedene, zulässige Geometrien. Insgesamt 100 Generationen lang wurde optimiert. Die Gesamtzahl der Zielfunktionsauswertungen ist größer als 1000, da auch von Entwürfen, die eine Nebenbedingung verletzen, eine Bewertung vorgenommen wurde. Die Veränderungen der Geometrien nehmen im Laufe der Optimierung ab, da die Wahrscheinlichkeit großer Mutationen von Generation zu Generation abnimmt (Abb.6.3 (a)). Das gefundene Minimum ist zwar um 1\% besser als das für die Ausgangsstruktur (A) mit SQP erzielte Ergebnis, aber nicht besser als das für die Ausgangsstruktur (B) gefundene Minimum mit einer Ausfallzeit von $P_{d}=1.30 \%$.

Das Adaptive Simulated Annealing benötigt für das Erreichen des Minimums für Ausgangsstruktur (A) 1662 Schritte (Abb.6.3 (b)), für (B) 2801 Schritte (Abb.6.3 (c)). Mit der Ausgangsstruktur (B) benötigt ASA somit etwa 20 mal soviel Auswertungen 


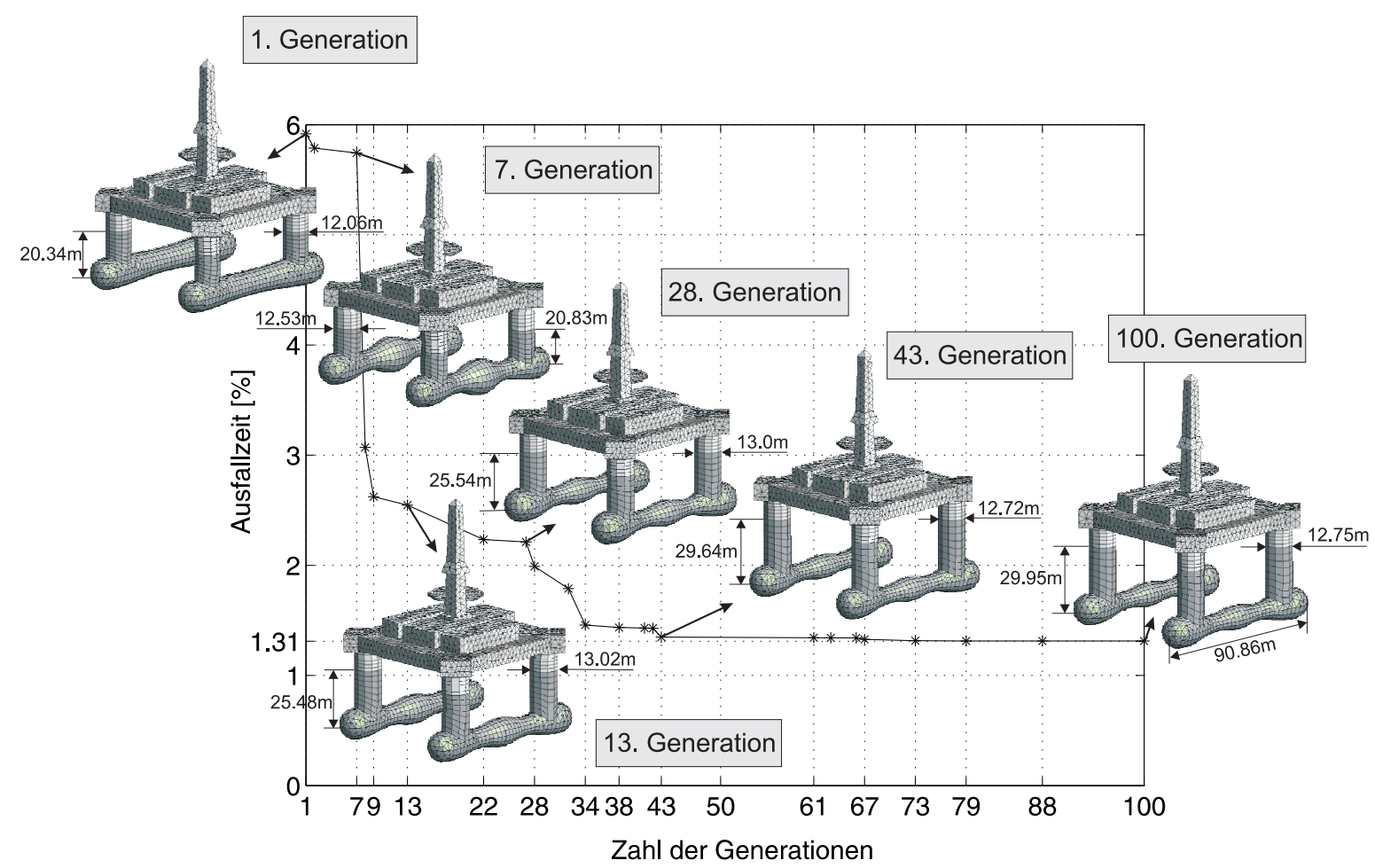

Abbildung 6.4: Entwicklung von Geometrie und Zielfunktion im Laufe der hydrodynamischen Formoptimierung mit einem Genetischen Algorithmus

der Zielfunktion wie SQP. Das gefundene Minimum ist mit einer minimierten Ausfallzeit von $P_{d}=1.26 \%$ das Beste. Insgesamt scheint ASA seltener in einem lokalen Minimum stecken zu bleiben (siehe auch Abschnitt 4.5.2). Wie Abb.6.3 (b) und (c) zeigen, macht ASA auch gegen Ende der Optimierung noch größere Sprünge. Diese Fähigkeit ist verantwortlich dafür, dass ASA öfters bessere lokale Minima als andere Optimierungsalgorithmen findet.

Die Zielfunktionswerte für die optimierten Strukturen SQP (B), ASA (A), ASA (B) und GA unterscheiden sich nur geringfügig (siehe Tab.6.2). Nur die von der Ausgangsstruktur (A) gestartete Optimierung mit SQP ergibt einen deutlich schlechteren Zielfunktionswert. Eine eingehende Untersuchung zeigt auf, ob hier tatsächlich unterschiedliche lokale Minima aufgefunden wurden. Da sich die Ergebnisse im 4-dimensionalen Variablenraum nur schwer visualisieren lassen, wurde ein Schnitt in $x_{1}-x_{2}$-Ebene durchgeführt. Die Variablen $x_{1}$ und $x_{2}$ haben einen größeren Einfluss auf den Zielfunktionswert als $x_{3}$ und $x_{4}$. Deshalb wurden die Werte von $x_{3}$ mit $60 \mathrm{~m}^{2}$ und $x_{4}$ mit $189 \mathrm{~m}^{2}$ festgehalten.

Wie Abb. 6.5 verdeutlicht, liegen SQP (A) und die Gruppe aus SQP (B), ASA (A), ASA (B) und GA tatsächlich auf unterschiedlichen Seiten einer „Rippe“ des „Zielfunktionsgebirges". Die „Rippe“ wird durch die Resonanzstelle der Tauchbewegung verursacht. Da die Übertragungsfunktion der Tauchbewegung an diskreten Frequenzen ausgewertet wird, schwanken die maximalen Amplituden im Resonanzpeak. Diese Schwankungen verursachen die separaten Spitzen entlang der Rippe. 

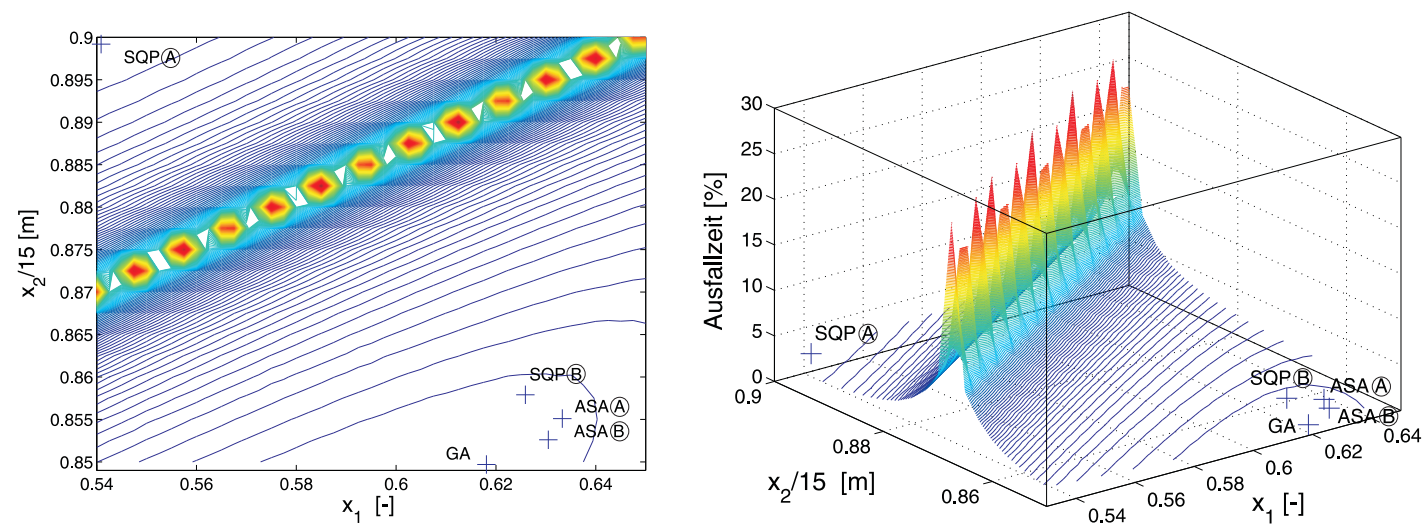

Abbildung 6.5: Darstellung der gefundenen Optima im Zielfunktionsraum für $x_{3}=$ $60 \mathrm{~m}^{2}$ und $x_{4}=189 \mathrm{~m}^{2}$

Die Gruppe von optimalen Lösungen (SQP (B), ASA (A), ASA (B), GA) liegt - zumindest im gezeigten Schnitt der $x_{1}-x_{2}$-Ebene - in einer flachen Mulde der Zielfunktion. Die Lösungen beschreiben vermutlich alle das gleiche lokale Minimum. Die geringen Unterschiede in der Geometrie sind auf numerische Ungenauigkeiten der hydrodynamischen Analyse und unterschiedliche Implementierungen der Abbruchkriterien in den Optimierungsalgorithmen zurückzuführen. Zusammenfassend belegt die Analyse die Abhängigkeit der Ergebnisse der lokalen SQP Strategie vom Ausgangspunkt und die Existenz mehrerer lokalen Minima.

Der Erfolg der hydrodynamischen Formoptimierung zeigt sich sowohl in den Übertragungsfunktionen der Tauchbewegung (Abb.6.6) als auch in den Übertragungsfunktionen der Stampfbewegung (Abb.6.7). Für beide Ausgangsstrukturen wirkt sich die große Amplitude der Übertragungsfunktion besonders im Bereich der mittleren Wellenfrequenz mit $0.4<\omega<0.7$ nachteilig auf die zu erwartende Ausfallzeit aus, da der Seegang in diesem Bereich die meiste Energie besitzt. Obwohl die Frequenzen der Tauchresonanz bei der optimierten Strukturen leicht erhöht wurden, sind die Amplituden der Tauchbewegung in diesem Frequenzbereich um mehr als die Hälfte verringert. Durch die Formoptimierung wurden auch die Amplituden der Stampfbewegung im Bereich der Wellenfrequenz mit $0.4<\omega<1.0$ deutlich reduziert. Im Bereich der Auslöschungsfrequenz $(\omega=0.3 \sim 0.4 \mathrm{rad} / \mathrm{s})$ treten Stampfbewegungen mit endlichen Amplituden auf, die im Falle ohne Zähigkeit eigentlich verschwinden müssten. Dies liegt an den Diskretisierungsfehlern im Frequenzbereich, die bei der numerischen Berechnung infolge der endlichen Zahl von Wellenfrequenzen verursacht werden.

Für die Optimierung mit der Ausgangsstruktur (B) werden in Abb. 6.8 Geometrien und Grenzkurven der zulässigen signifikanten Wellenhöhen $H_{s, z u l}$ dargestellt. Die Unterschiede in den Hauptabmessungen sind gering und die Grenzkurven sind für alle drei optimierten Entwürfe fast identisch. Charakteristisch für alle optimierten Konstruktionen ist die Vergrößerung des Tiefgangs und die Umverteilung der Verdrängung von der Pontonmitte zu den Pontonenden. Ersteres verringert deutlich die vertikale Erregerkraft, da die getauchten Pontons weiter aus dem Wirkungsbereich der Wellen 

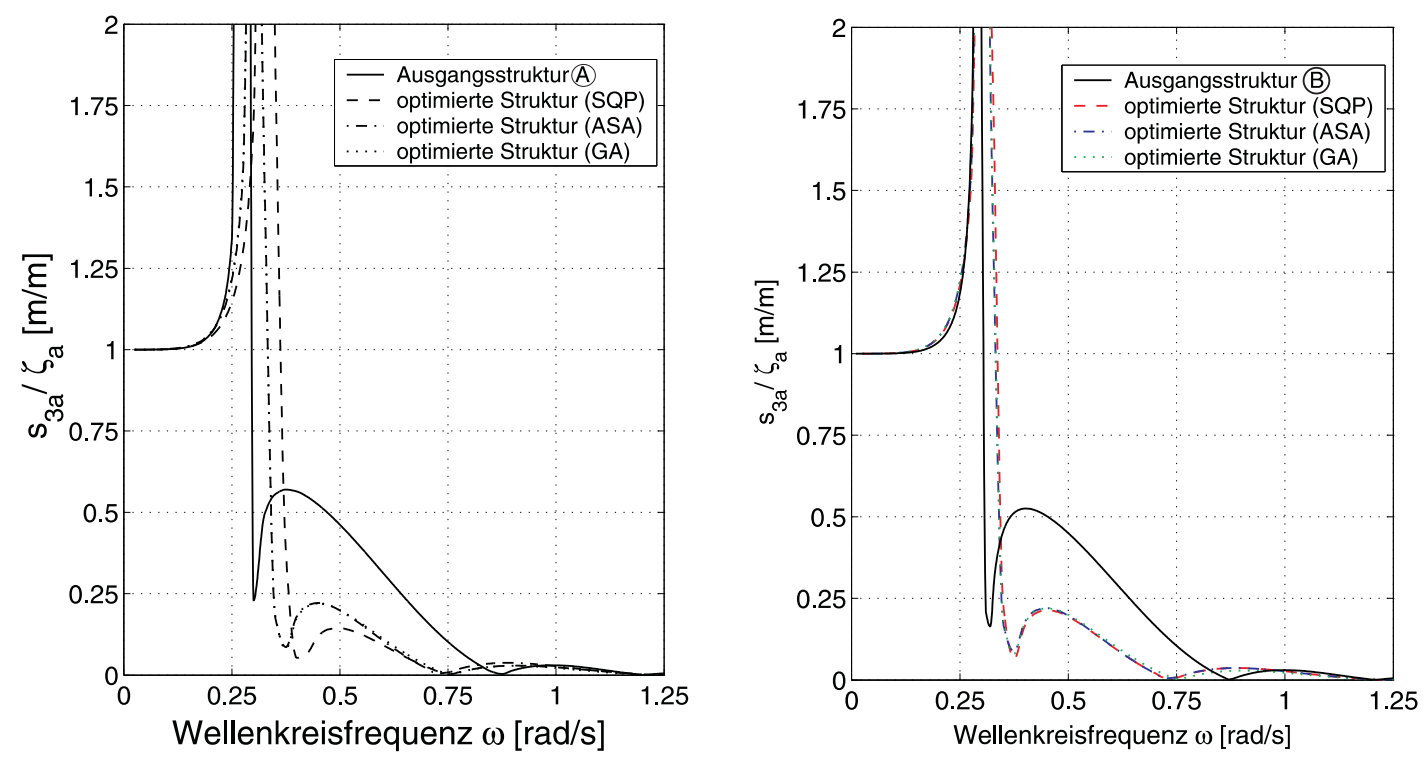

Abbildung 6.6: Vergleich der Übertragungsfunktionen der Tauchbewegung bei unterschiedlichen Ausgangsstrukturen
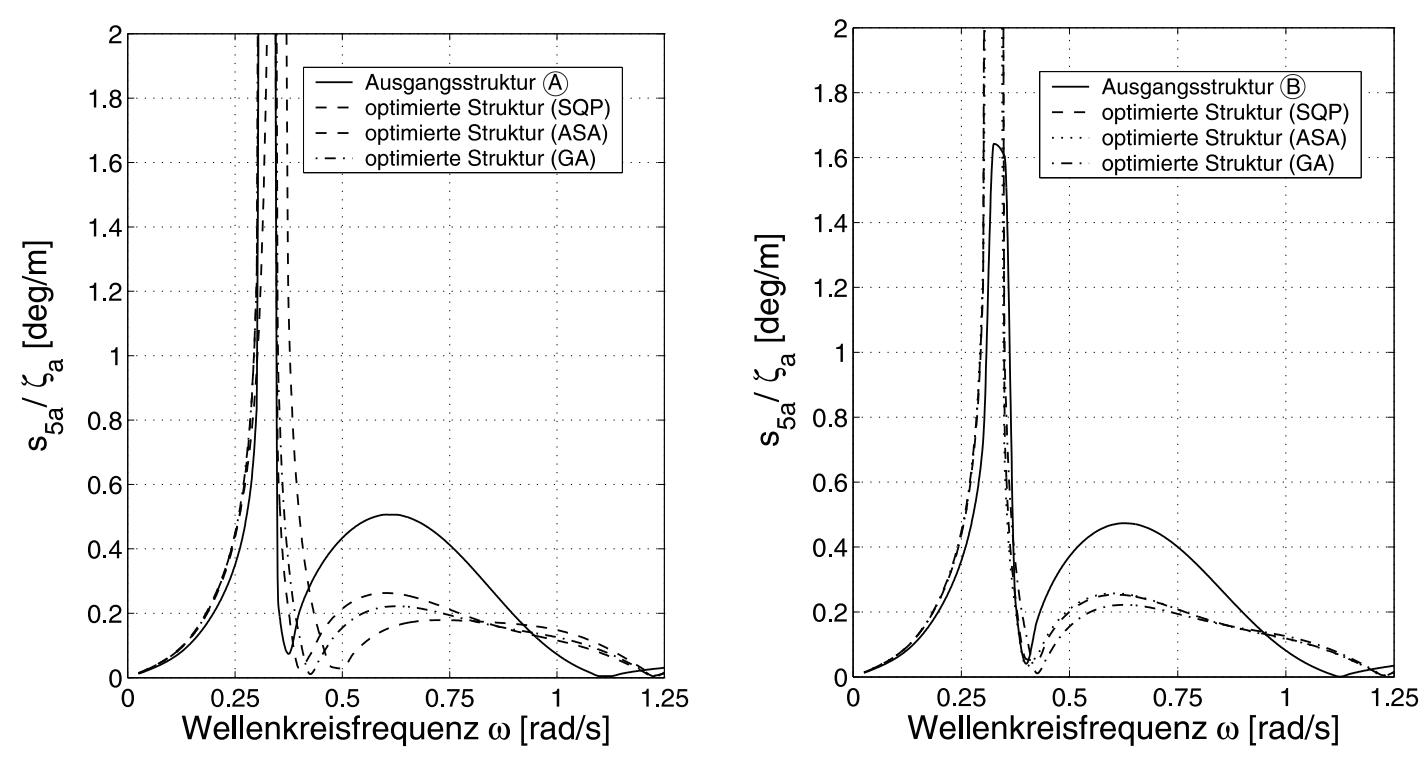

Abbildung 6.7: Vergleich der Übertragungsfunktionen der Stampfbewegung bei unterschiedlichen Ausgangsstrukturen 


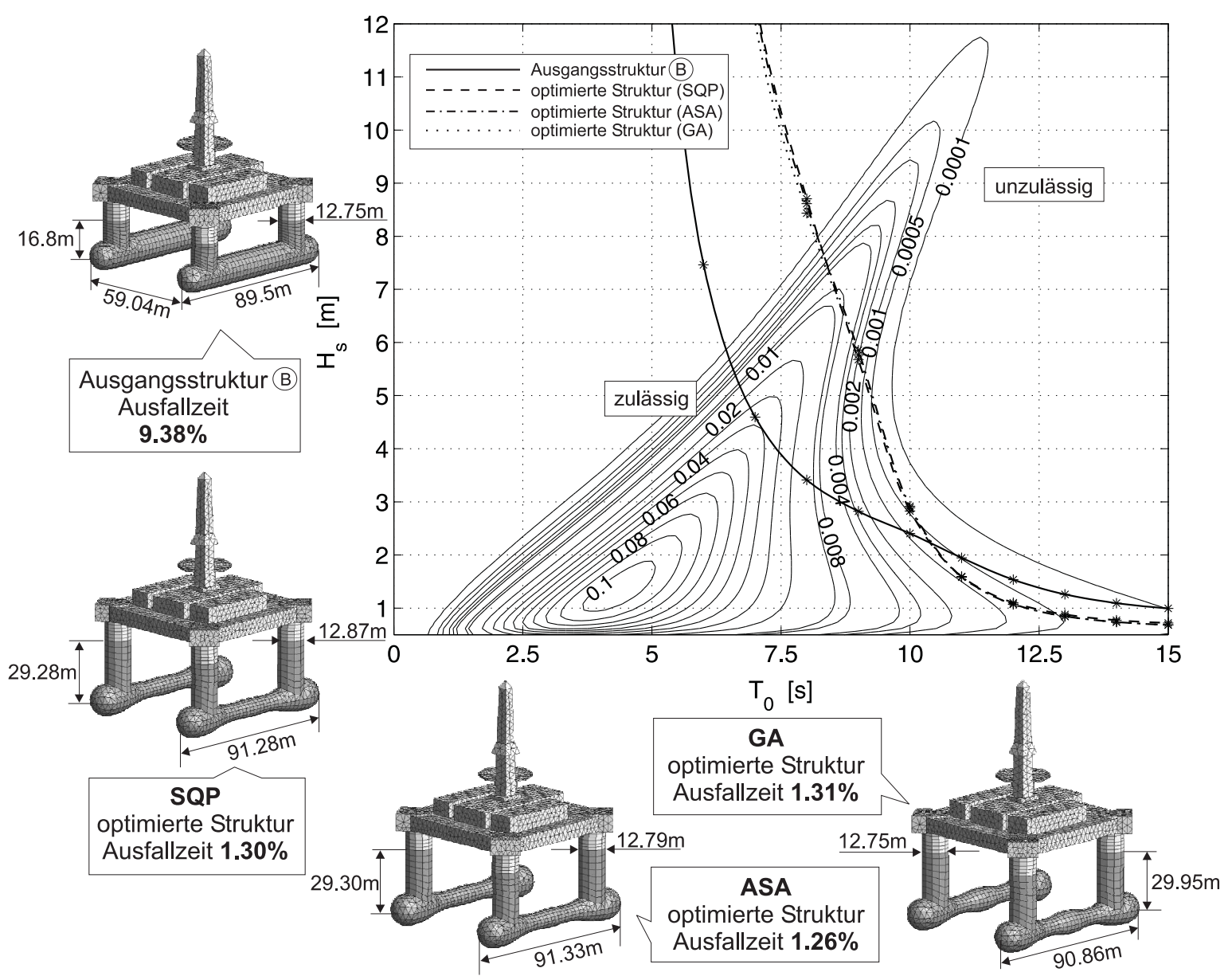

Abbildung 6.8: Vergleich der Geometrien und Grenzkurven der zulässigen signifikanten Wellenhöhen (Optimierung mit Ausgangsstruktur (B): Seegangsrichtung von vorne

herausgeschoben werden.

\subsubsection{Halbtaucher mit 9 freien Variablen}

Optimierungsaufgaben mit vielen freien Variablen treten z.B. dann auf, wenn der Bereich „guter" Entwurfslösungen der Optimierungsaufgabe nicht durch bereits vorhandene Entwurfserfahrung eingeschränkt werden kann. Um die Zuverlässigkeit sowie die Leistung der Optimierungsstrategien bei wachsender Anzahl der freien Variablen (und auch Nebenbedingungen) zu untersuchen, wird ein Halbtaucher mit Verdrängung von $\triangle=30,000 m^{3}$ entwickelt, der wiederum aus vier Säulen und zwei Pontons besteht. Die Querschnittsfläche des Pontons ist rechteckig. Die geeigneten hydrodynamischen Massenbeiwerte $C_{a}$ für Quader können in Abhängigkeit des Seitenverhältnisses aus Diagrammen entnommen werden (siehe z.B. Clauss et al. (1992)). Eine Pontonhälfte wird in drei Abschnitte unterteilt: Mittelsektion, Hauptsektion unter der Säule und Endsektion (Abb. 6.9). Als freie Variable sind die folgenden Formparameter ausgewählt worden: 


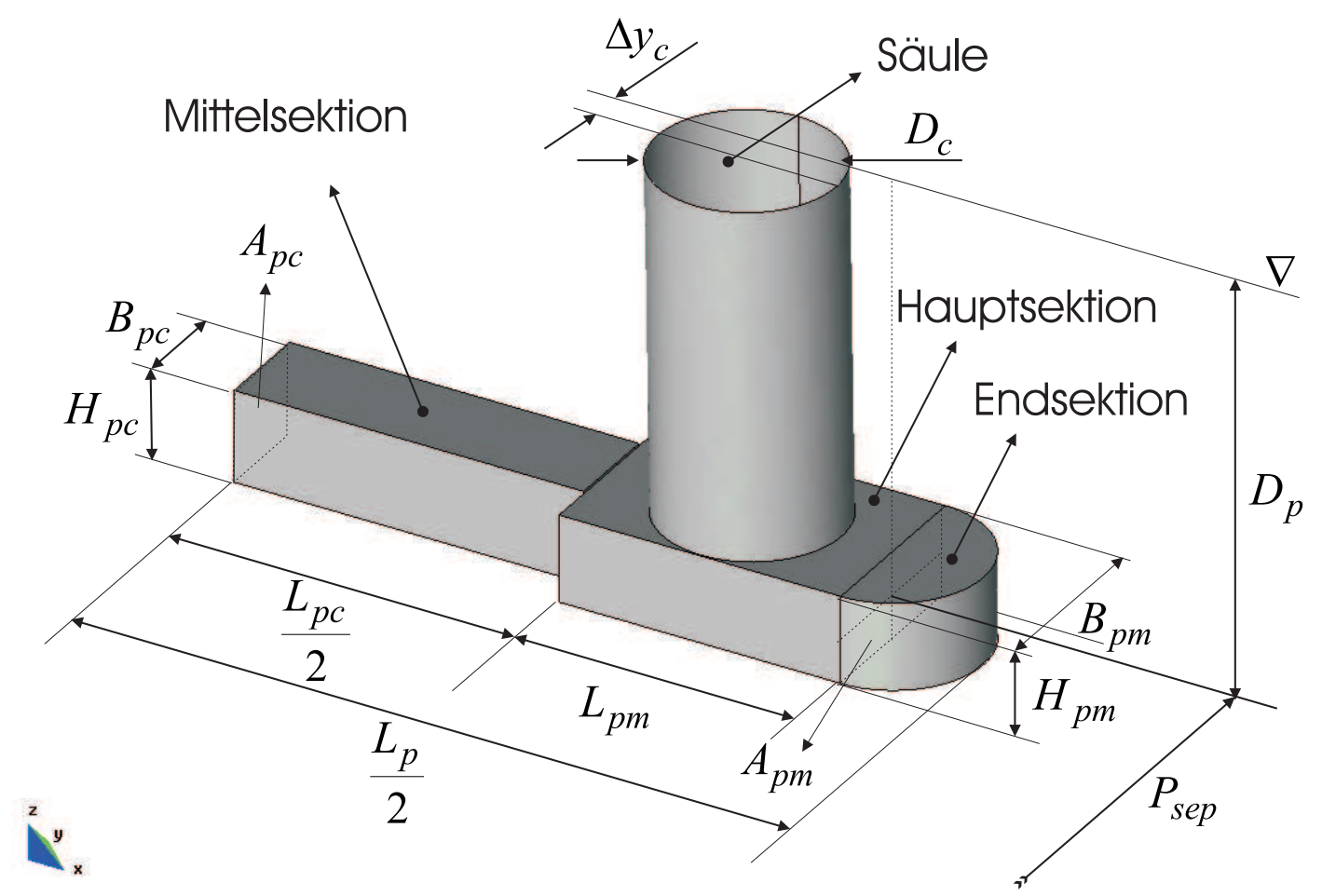

Abbildung 6.9: Definition der Formparameter für den Halbtaucher mit 9 freien Variablen

- der Abstand zwischen beiden Pontonachsen: $x_{1}=P_{s e p}$

- die Tauchtiefe der Pontonachse: $x_{2}=D_{p}$

- das Verhältnis der Querschnittsflächen von Hauptsektion und Mittelsektion: $x_{3}=A_{p m} / A_{p c}$

- das Verhältnis der Länge von Hauptsektion und Mittelsektion: $x_{4}=L_{p m} / L_{p c}$

- das Verhältnis von Breite zu Höhe der Hauptsektion: $x_{5}=B_{p m} / H_{p m}$

- das Verhältnis von Breite zu Höhe der Mittelsektion: $x_{6}=B_{p c} / H_{p c}$

- der Durchmesser der Säulen: $x_{7}=D_{c}$

- die Querverschiebung der Säulen an der Wasseroberfläche (nach Innen): $x_{8}=\triangle y_{c}$ und

- die Länge des Pontons: $x_{9}=L_{p}=L_{p c}+2 L_{p m}+B_{p m}$

Für die Optimierung werden auch geometrische und hydrostatische Nebenbedingungen angenommen: 

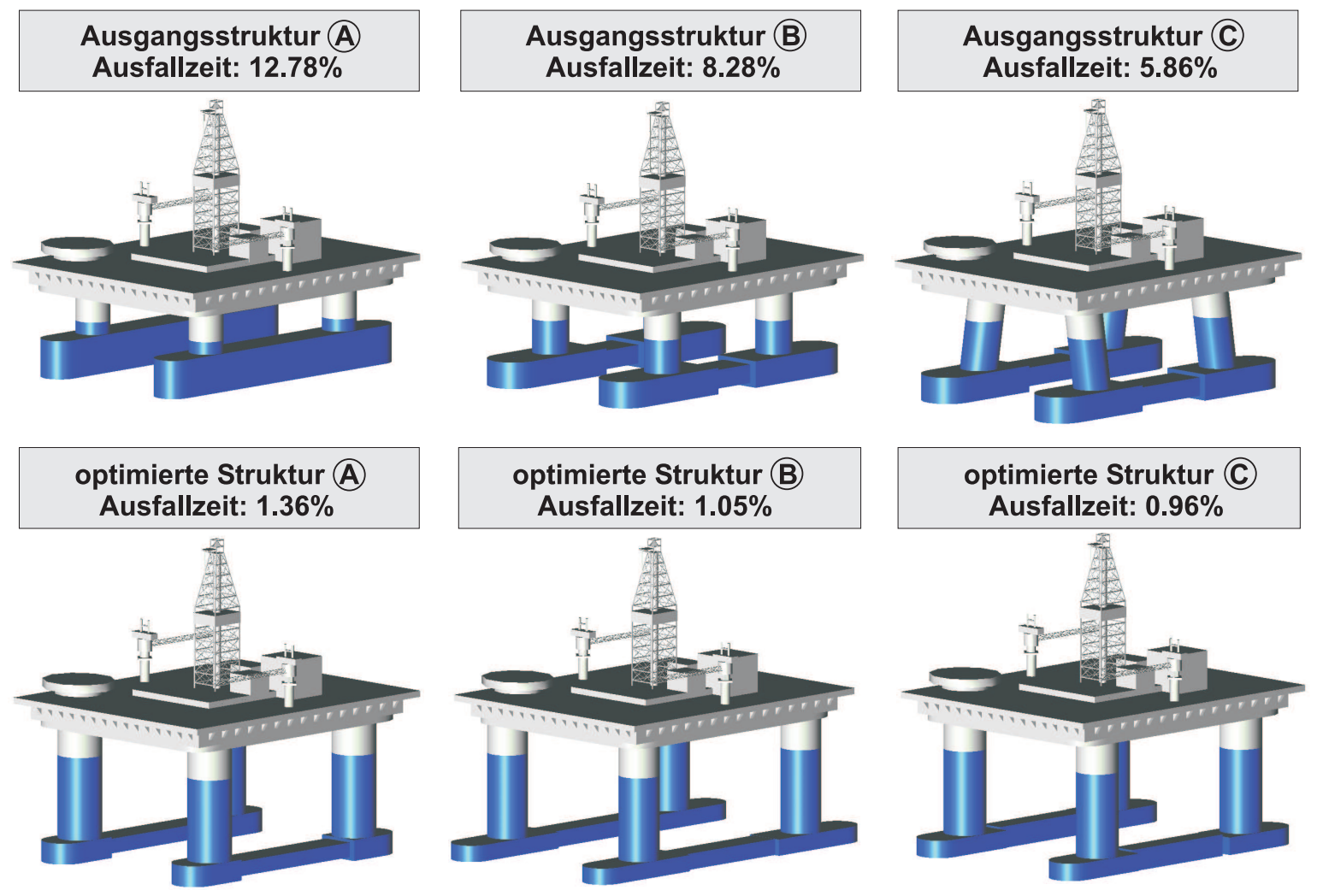

Abbildung 6.10: Optimierung eines Halbtauchers mit 9 freien Variablen: Ergebnis für die lokale SQP Strategie

- die minimale metazentrische Höhe in Quer- und Längsrichtung soll größer als $1 \mathrm{~m}$ sein: $\overline{G M}_{T 0} \geq 1.0 \mathrm{~m}, \overline{G M}_{L 0} \geq 1.0 \mathrm{~m}$

- die minimale Querschnittsfläche des Pontons für die Mittelsektion darf nicht weniger als $50 \mathrm{~m}^{2}$ sein: $A_{p c}>50 \mathrm{~m}^{2}$

- der Durchmesser der Säulen soll kleiner als die Breite und die Länge der Haupsektion sein: $B_{p m}>D_{c}, L_{p m}>D_{c}$

Optimiert werden soll die Unterwassergeometrie des Halbtauchers, wobei die statistisch zu erwartende Ausfallzeit für das Seegebiet Haltenbanken minimiert wird. Bei der numerischen Berechnung für die Zielfunktion wird hier auch die Zähigkeit nicht berücksichtigt. Die Resonanzspitze der Übertragungsfunktion wird jedoch auf $2[\mathrm{~m} / \mathrm{m}]$ begrenzt, um verfälschende Einflüsse des zufälligen Auftretens extrem großer Zielfunktionswerte im Resonanzfall auszuschalten. Die Optimierungen für das lokale Suchverfahren SQP wurden mit 3 Ausgangsstrukturen durchgeführt (Abb. 6.10, siehe auch Tab. 6.3). Die Ausgangsstrukturen sind so ausgewählt, dass sie möglichst unterschiedliche Formparameter und damit verschiedene Seegangseigenschaften besitzen. Alle Ausgangsstrukturen sind zulässige Entwürfe. Die Ausgangsstruktur (C) besitzt die kleinste Ausfallzeit (5.86\%, siehe Abb.6.10). 


\begin{tabular}{|l|lllllllll|}
\hline \multicolumn{10}{|c|}{ Ausgangsstrukturen } \\
\hline freie & $x_{1}$ & $x_{2}$ & $x_{3}$ & $x_{4}$ & $x_{5}$ & $x_{6}$ & $x_{7}$ & $x_{8}$ & $x_{9}$ \\
Variable & {$[\mathrm{m}]$} & {$[\mathrm{m}]$} & {$[\cdot]$} & {$[\cdot]$} & {$[\cdot]$} & {$[\cdot]$} & {$[\mathrm{m}]$} & {$[\mathrm{m}]$} & {$[\mathrm{m}]$} \\
\hline Typ (A) & 50.0 & 10.0 & 1.0 & 1.0 & 1.0 & 1.0 & 11.0 & 0.0 & 100.0 \\
Typ (B) & 50.0 & 15.0 & 2.0 & 2.0 & 2.0 & 2.0 & 12.0 & 0.0 & 100.0 \\
Typ (C) & 50.0 & 20.0 & 2.0 & 1.0 & 2.0 & 2.0 & 12.0 & 2.5 & 100.0 \\
\hline \multicolumn{10}{|c|}{ optimierte Strukturen: SQP } \\
\hline SQP (A) & 58.31 & 30.0 & 1.92 & 0.29 & 2.13 & 2.24 & 14.35 & 0.02 & 93.38 \\
SQP (B) & 59.83 & 30.0 & 1.41 & 0.82 & 2.50 & 1.83 & 13.28 & 0.54 & 119.95 \\
SQP (C) & 59.51 & 30.0 & 1.49 & 0.58 & 2.50 & 1.10 & 13.85 & 0.00 & 102.63 \\
\hline
\end{tabular}

Tabelle 6.3: Formparameter der gewählten Ausgangsstrukturen und optimierter Strukturen mit dem lokalen Optimierungsalgorithmus SQP

Wie Abb. 6.10 und Abb. 6.11 zeigen, konnte die zu erwartende Ausfallzeit durch die Formoptimierung mit der lokalen SQP Suchstrategie um $80 \%$ vermindert werden. Dabei führen die drei Ausgangsstrukturen jeweils zu deutlich verschiedenen Optima (siehe Tab.6.3), obwohl die Optimierungen unter gleichen Bedingungen (z.B. mit gleichen Toleranzen der Schrittweite $1 e^{-4}$ und Abbruchkriterien $1 e^{-3}$ ) durchgeführt wurden. Die Gründe, wann welche Ausgangsstruktur zu welchem Ergebnis führt, können nur detaillierte Untersuchungen des gesamten Entwurfsraumes ergeben. Diese sind jedoch so aufwendig, dass sie in der Praxis nicht durchgeführt werden können. Mit der Ausgangsstruktur (C) erzielt der SQP Algorithmus die beste optimierte Struktur mit einer Ausfallzeit von $0.96 \%$, während mit der Ausgangsstruktur (A) die minimierte Ausfallzeit immer noch $1.36 \%$ beträgt. Einige der durch den SQP Algorithmus gefundenen Formparameter liegen direkt an den Grenzwerten, die vor der Optimierung als Oberund Untergrenze definiert sind. Bei allen drei optimierten Strukturen ist z.B. der Tiefgang mit $D_{p}=30 \mathrm{~m}$ gleich. Der optimierte Abstand zwischen beiden Pontonachsen $P_{\text {sep }}$ erreicht fast den maximalen Wert von 60m. Für die Querverschiebung der Säulen an der Wasseroberfläche liegen die Formparameter an der Untergrenze Null, d.h. die Säulen der optimierten Strukturen stehen fast senkrecht.

Abb. 6.11 zeigt den typischen Verlauf der Optimierung unter Anwendung der lokalen SQP Strategie. Dargestellt ist die Entwicklung der zu erwartenden Ausfallzeit als Funktion der Anzahl der Optimierungsschritte. Der SQP Algorithmus findet, ausgehend von der Ausgangsstruktur (B), bereits nach 30 Schritten ein Minimum der Zielfunktion. Mit der Ausgangsstruktur (A) wird dagegen das Minimum erst nach ca. 80 Schritten gefunden, da Nebenbedingungen verletzt wurden. Jeder Schritt bei SQP entspricht einer Auswertung der Zielfunktion. Typisch für alle lokalen Suchstrategien ist das Suchen einer Abstiegsrichtung im Variablenraum während der ersten Schritte (110) und der daraufhin folgende schnelle Erfolg der Suche: das SQP Verfahren benötigt hierbei 10 Funktionsauswertungen, um den Gradienten der Zielfunktion (mit 9 freien 


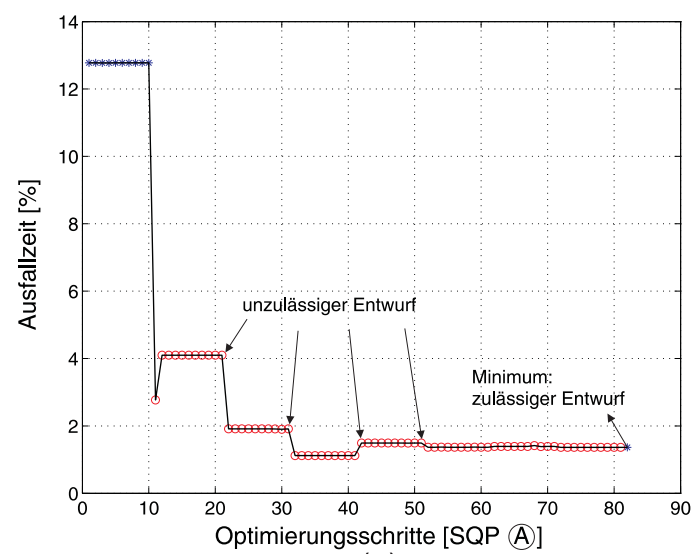

(a)

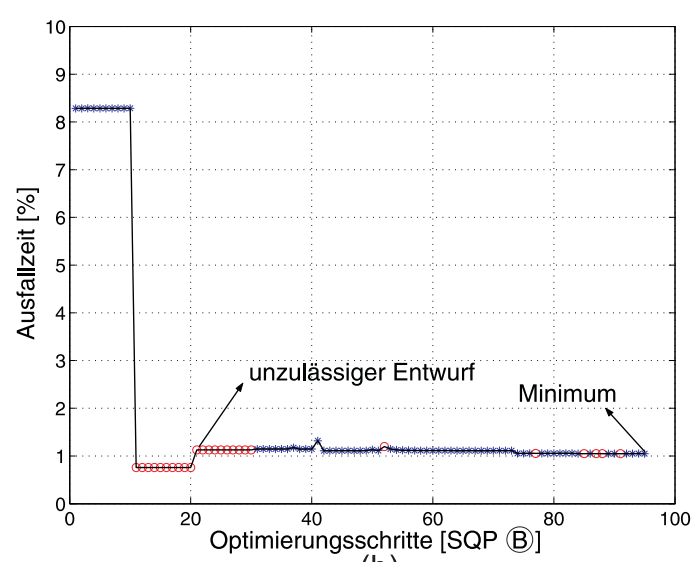

(b)

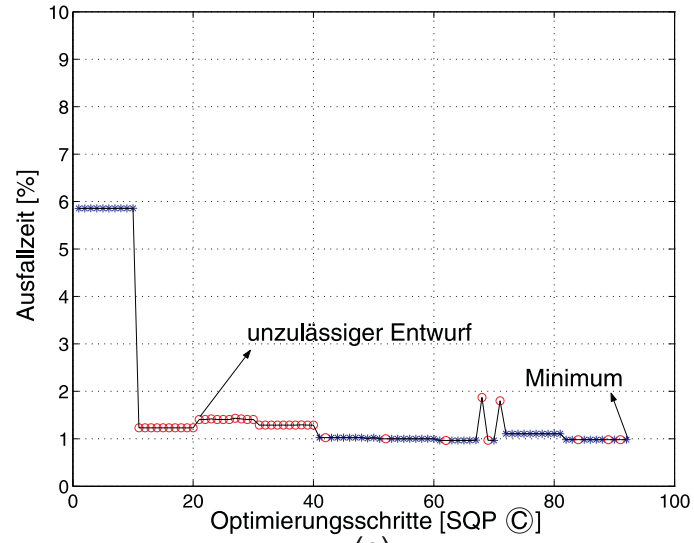

(c)

Abbildung 6.11: Optimierungsschritte und Änderung der Zielfunktion für den SQPAlgorithmus: (a) mit der Ausgangsstruktur (A) (b) mit der Ausgangsstruktur (B) (c) mit der Ausgangsstruktur (C)

Variablen) abzuschätzen. Wenn ein unzulässiger Entwurf entsteht, sucht das Verfahren nach einer neuen zulässigen Richtung. Eine ganze Reihe von zusätzlichen Funktionsauswertungen ist notwendig, um das gefundene Minimum genauer einzugrenzen und zu bestätigen. Mit der Ausgangsstruktur (C) findet der SQP Algorithmus z.B. nach 40 Funktionsauswertungen ein Minimum der Zielfunktion, wobei viermal die Gradienten der Zielfunktion abgeschätzt werden. Zu beobachten ist, dass der Rechenaufwand (Anzahl der Auswertungen der Zielfunktion) im Vergleich zum vorigen Optimierungsproblem mit 4 freien Variablen nicht größer wird (siehe Tab.6.2), obwohl die Anzahl der freien Variablen und der Nebenbedingungen vergrößert wurde.

Die gleiche Optimierungsaufgabe wurde auch mit den globalen Strategien, Genetischer Algorithmus (GA), Adaptive Simulated Annealing (ASA) und Branch-and-Bound Algorithmus (BaB) gelöst. Tab.6.4 fasst die Ergebnisse zusammen. Die gefundenen Minima und die Anzahl der benötigten Zielfunktionsbewertungen in Tab. 6.4 zeigen, dass die durch die globalen Optimierungsalgorithmen gefundenen Minima bis auf BaB besser als die mit SQP erzielten sind. Bezüglich des Rechenaufwandes und der Leistung 


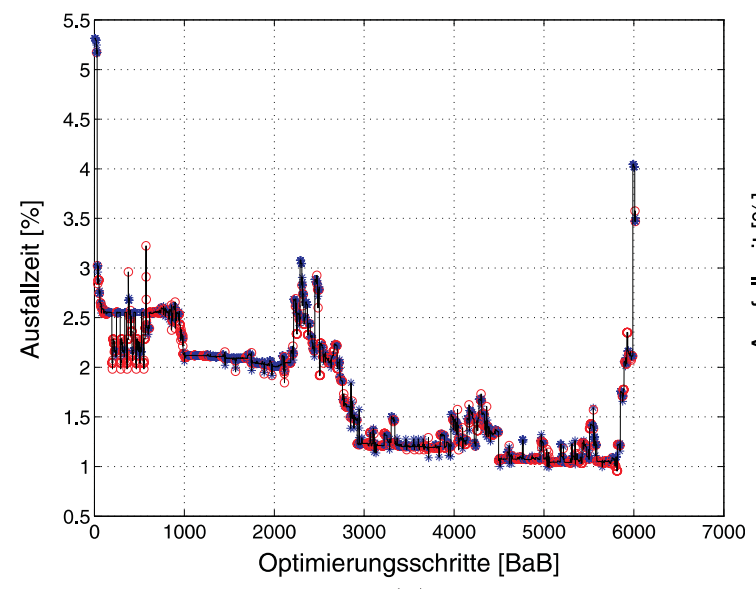

(a)

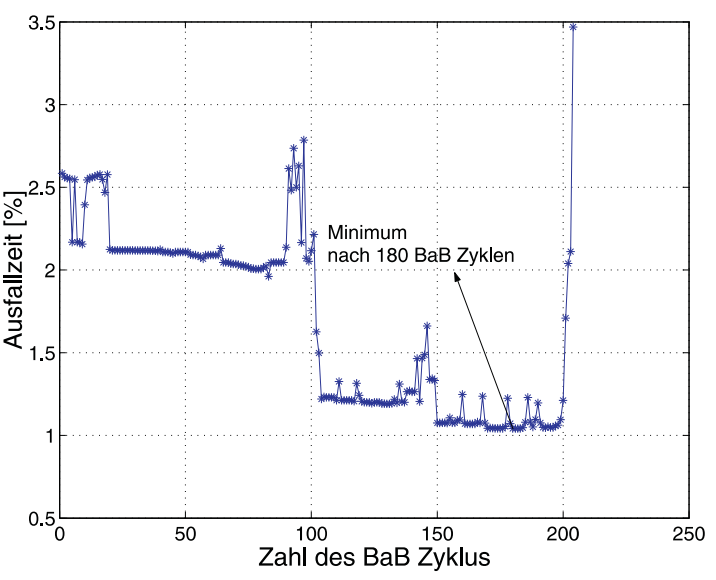

(b)

Abbildung 6.12: Verlauf der Optimierung für den BaB Algorithmus: Änderung der Zielfunktion auf Optimierungsschritte (a) Änderung der Zielfunktion auf BaB Zyklen

\begin{tabular}{|c|c|c|}
\hline \multicolumn{3}{|c|}{ Begrenzung des RAOs auf $2[\mathrm{~m} / \mathrm{m}]$} \\
\hline Algorithmen & Anzahl der & $\begin{array}{l}\text { Ausfallzeit } \\
\text { in \% }\end{array}$ \\
\hline SQP (A) & 82 & 1.36 \\
SQP (B) & 95 & 1.05 \\
SQP C & 92 & 0.96 \\
GA & 1297 & 0.90 \\
ASA C & 2320 & 0.90 \\
BaB C) & 6019 & 1.04 \\
\hline
\end{tabular}

Tabelle 6.4: Vergleich der Ergebnisse der globalen Optimierungsalgorithmen mit der lokalen SQP Strategie

hat sich der $\mathrm{BaB}$ Algorithmus allerdings als am schlechtesten erwiesen. BaB benötigt mehr als 6000 Funktionsauswertungen und findet ein Minimum von $1.04 \%$ nach 180 BaB Zyklen (siehe Abb. 6.12). Das Minimum von SQP $\mathbb{C}$ ist hier sogar um $0.08 \%$ besser als das mit $\mathrm{BaB}$ erzielte. Obwohl der BaB Algorithmus vermutlich durch bessere Abstimmung von Steuerparametern noch bessere Ergebnisse erzielen könnte, wurde aufgrund des enormen Rechenaufwandes BaB nicht in der Formoptimierung hydrodynamisch kompakter Strukturen verwendet.

Der Genetische Algorithmus und das Adaptive Simulated Annealing benötigten zwar einen deutlich größeren Rechenaufwand, erzielen aber bessere Ergebnisse als die lokale Suchstrategie SQP. Wie in Abb. 6.13(a) dargestellt, benötigt der GA Algorithmus etwa 1300 Auswertungen der Zielfunktion (Tab.6.4). Die Zahl der Zielfunktionsauswertungen bei GA hängt vor allem von der maximalen Anzahl der zulässigen 


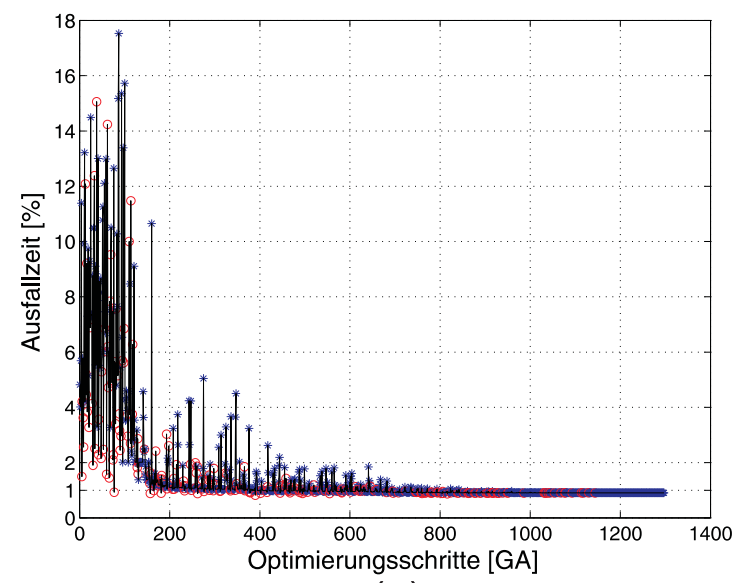

(a)

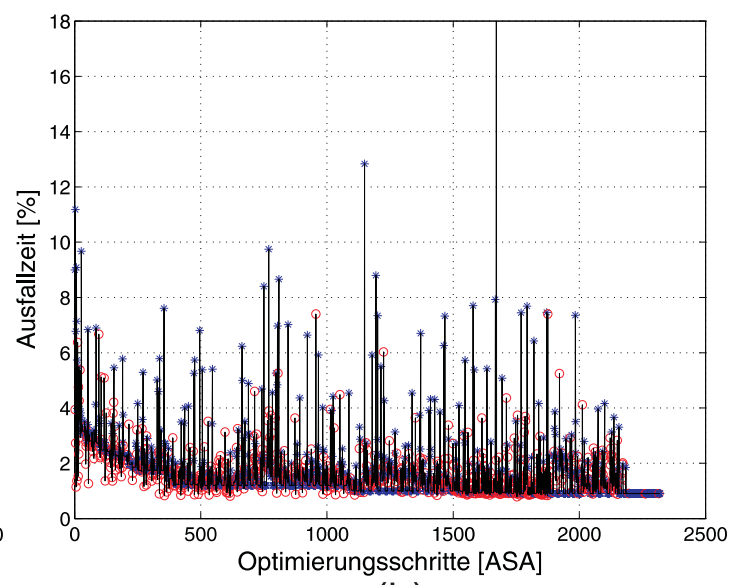

(b)

Abbildung 6.13: Optimierungsschritte und Änderung der Zielfunktion für den Genetischen Algorithmus (a) und für den Adaptive Simulated Annealing Algorithmus (b)

\begin{tabular}{|c|c|c|}
\hline \multicolumn{3}{|c|}{ Kombinierte Optimierungsstrategien } \\
\hline \multicolumn{2}{|c|}{ Begrenzung des RAOs auf $2[\mathrm{~m} / \mathrm{m}]$} \\
\hline Algorithmen & $\begin{array}{l}\text { Anzahl der } \\
\text { Zielfunktionsbewertungen }\end{array}$ & $\begin{array}{l}\text { Ausfallzeit } \\
\text { in } \%\end{array}$ \\
\hline GA + SQP & $1297(\mathrm{GA})+105(\mathrm{SQP})$ & 0.86 \\
ASA + SQP & $2320(\mathrm{ASA})+121(\mathrm{SQP})$ & 0.89 \\
\hline
\end{tabular}

Tabelle 6.5: Ergebnisse mit den kombinierten Optimierungsstrategien

Generationen ab. Da das Minimum innerhalb der 100 Generationen nicht nach der vorgegebenen numerischen Genauigkeit eingegrenzt werden konnte, wurde die Optimierung bis zur maximalen Zahl der Generationen durchgeführt. ASA braucht etwa 1000 Auswertungen der Zielfunktion mehr als GA, um das selbe Minimum zu lokalisieren (Abb. 6.13(b)).

Im Gegensatz zum Genetischen Algorithmus behält ASA bis zum Schluss die Fähigkeit, größere Schritte im Variablenraum zu unternehmen, obwohl auch hier die Wahrscheinlichkeiten für große Schritte im Laufe der Optimierung abnehmen. Aufgrund der großen Änderungen der freien Variablen wurden sehr häufig Nebenbedingungen verletzt. Im Laufe der Optimierung verwirft das ASA Verfahren die Kombination der freien Variablen, die Nebenbedingungen nicht erfüllen. Dies ist ein Grund für den größeren Rechenaufwand bei Optimierungsaufgaben mit mehreren Nebenbedingungen.

Mit einer Ausfallzeit von $P_{d}=0.90 \%$ haben die durch GA und ASA ermittelten optimierten Strukturen zwar fast den gleichen Zielfunktionswert, jedoch sind die gefundenen Formparameter leicht verschieden (Abb. 6.14). Bei der durch GA optimierten Struktur ist z.B. die Querverschiebung der Säulen an der Wasseroberfläche mit 3.33m größer als bei der durch ASA optimierten Struktur mit 0.73m (schrägstehende Säulen bei GA, siehe Abb. 6.14). 


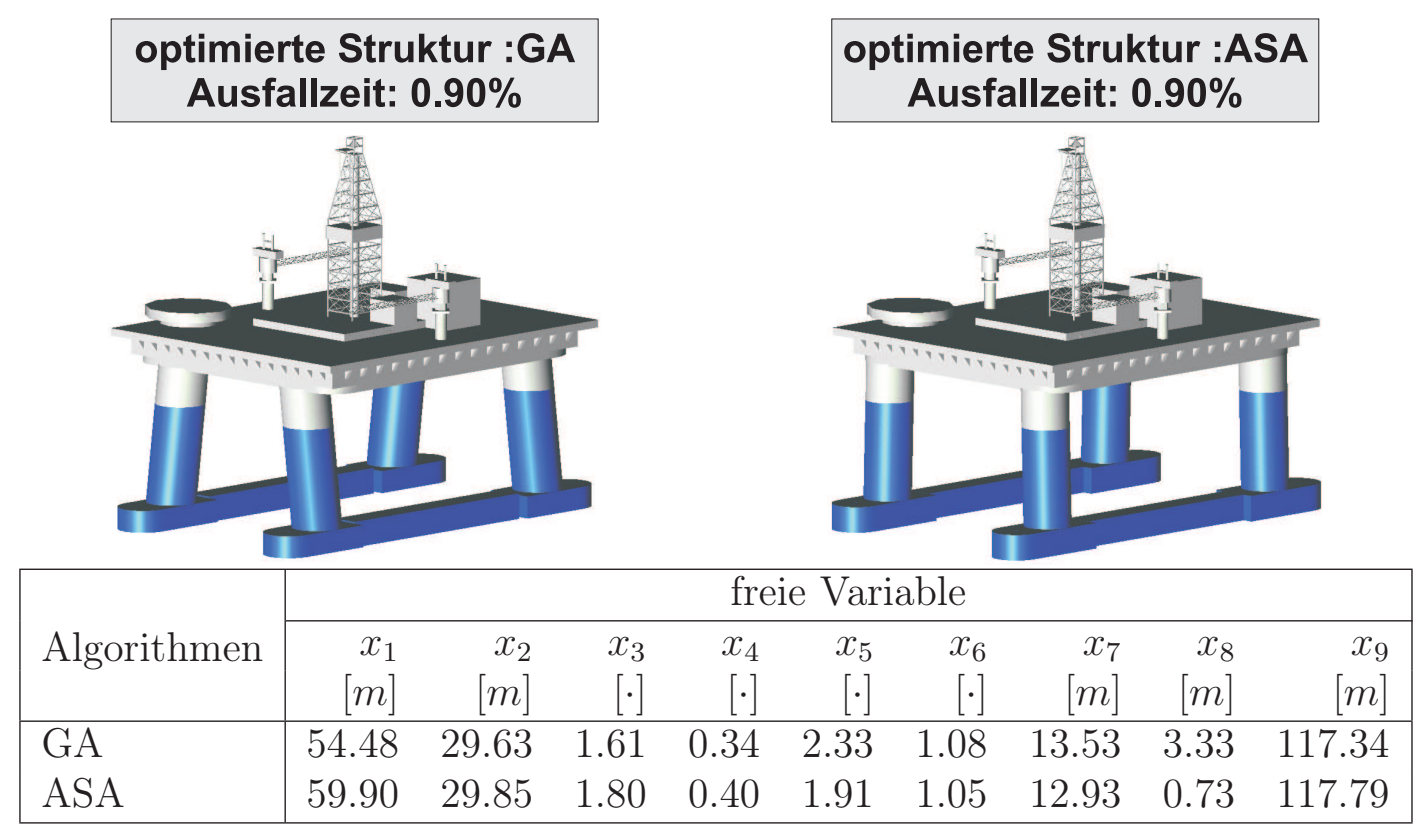

Abbildung 6.14: Ergebnisse der Optimierung eines Halbtauchers mit 9 freien Variablen mit den globalen Optimierungsalgorithmen (GA und ASA)

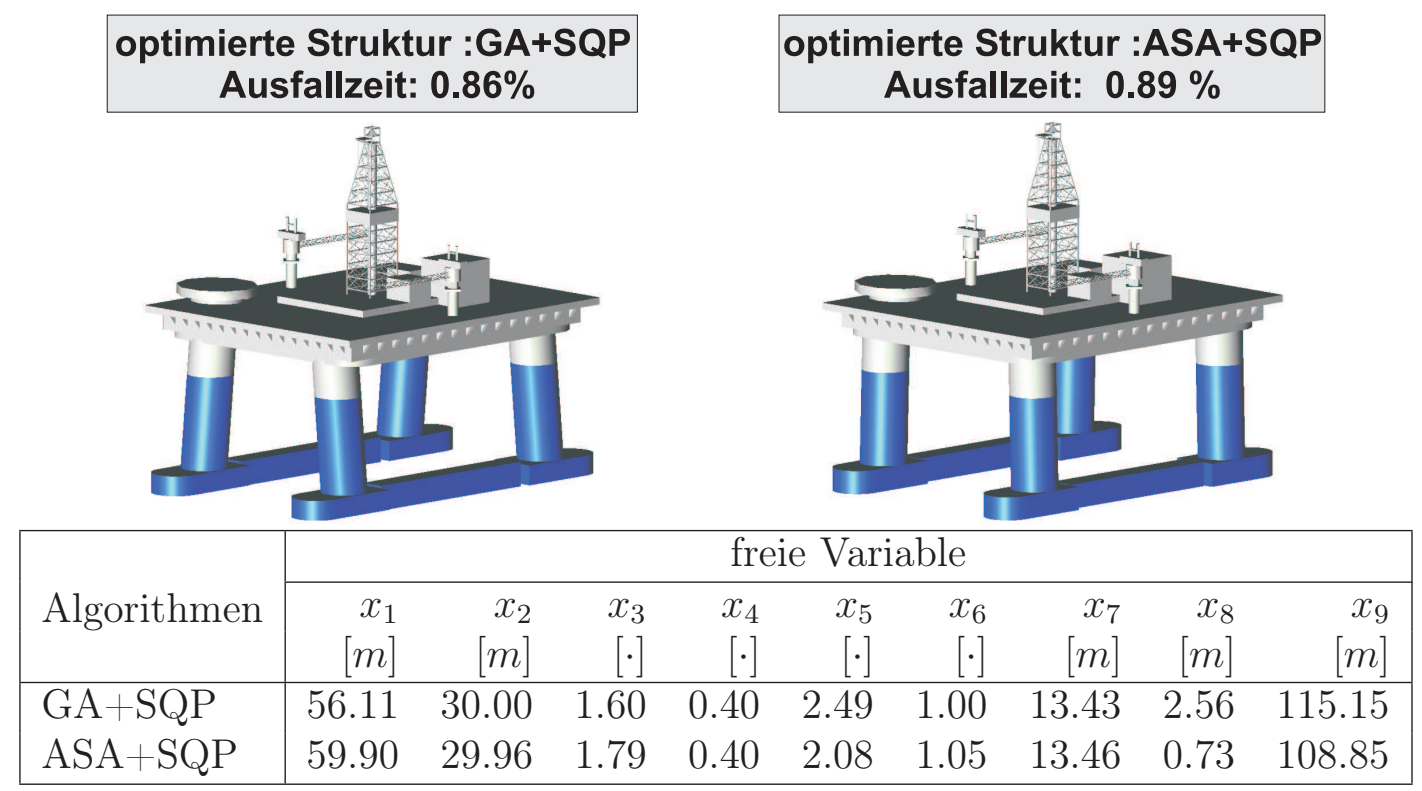

Abbildung 6.15: Ergebnisse der Optimierung eines Halbtauchers mit 9 freien Variablen mit den hybriden Optimierungsalgorithmen $(\mathrm{GA}+\mathrm{SQP}$ und $\mathrm{ASA}+\mathrm{SQP})$ 


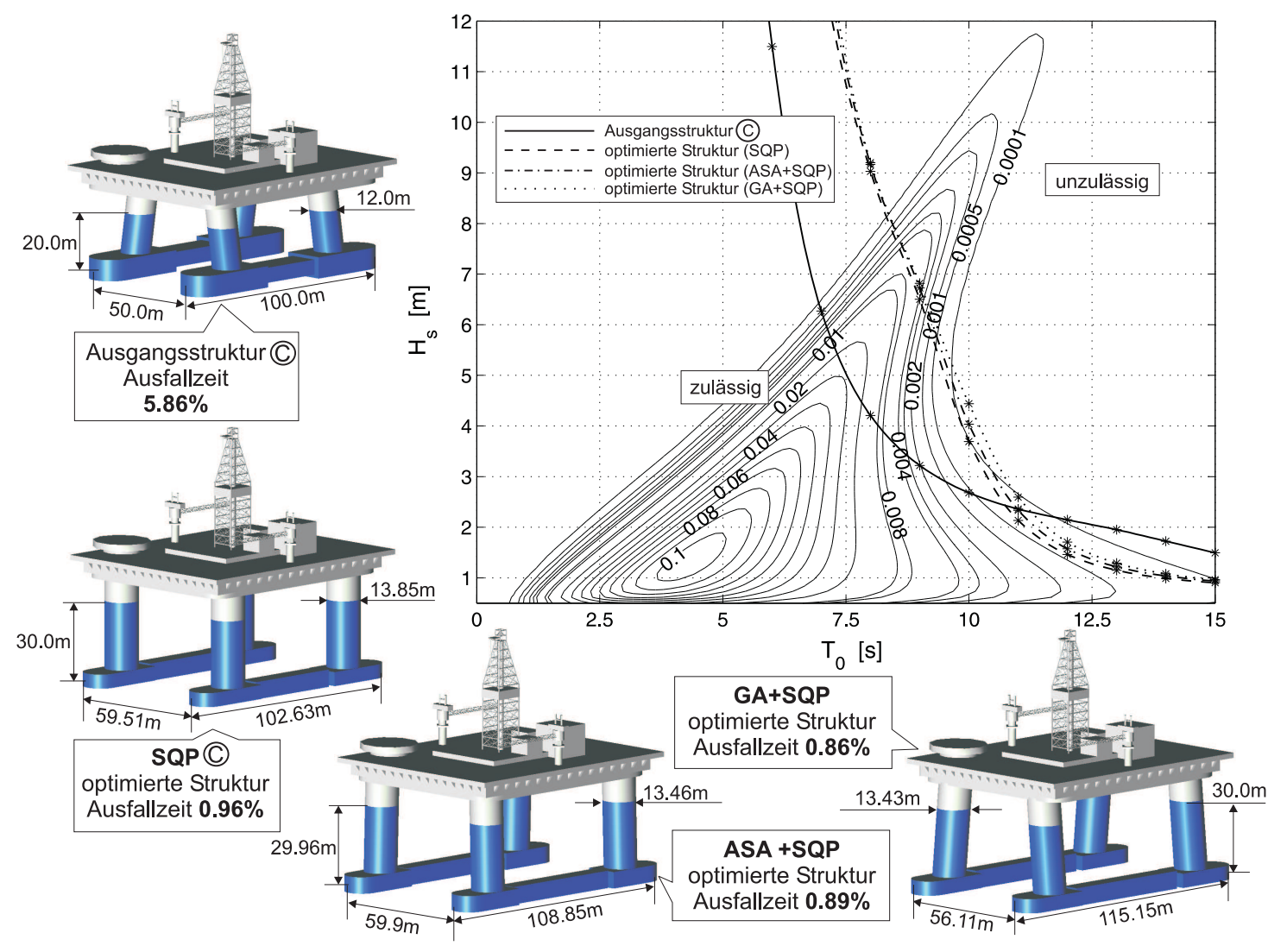

Abbildung 6.16: Vergleich der Geometrien und Grenzkurven der zulässigen signifikanten Wellenhöhen: Optimierungen mit Ausgangsstruktur $\mathbb{C}$ mit der Begrenzung der maximalen Amplituden auf $2[\mathrm{~m} / \mathrm{m}]$

In Abb.6.15 werden die Ergebnisse der kombinierten bzw. hybriden Optimierung dargestellt. Die beiden Minima konnten durch eine nachgeschaltete Optimierung mit der lokalen Suchstrategie SQP weiter verbessert werden. Das beste Minimum wurde ausgehend von der durch GA minimierten Struktur mit der Ausfallzeit von $0.86 \%$ erzielt. Mit der optimierten Struktur von ASA wurde allerdings nur eine geringe Verbesserung der Zielfunktion mit 0.01\% erreicht (siehe Tab.6.5).

Zum Vergleich wurden die Geometrien und die Grenzkurven der zulässigen signifikanten Wellenhöhen für die durch den lokalen (SQP (C) und die globalen (bzw. hybriden) Algorithmen (ASA+SQP, GA+SQP) optimierten Entwürfe in Abb. 6.16 dargestellt. Bei allen optimierten Strukturen werden die Grenzkurven der zulässigen Wellenhöhen deutlich nach rechts verschoben und damit der Bereich der zulässigen Seegangszustände erweitert.

\subsubsection{Effekte der Zähigkeit auf die Übertragungsfunktion}

Bei der Analyse hydrodynamisch transparenter Konstruktionen ist es möglich, den Zähigkeitseinfluss auf die Formoptimierung zu berücksichtigen, weil das entwickelte TFC-Programm die linearisierte zähigkeitsbedingte Widerstandskraft einbezieht (sie- 


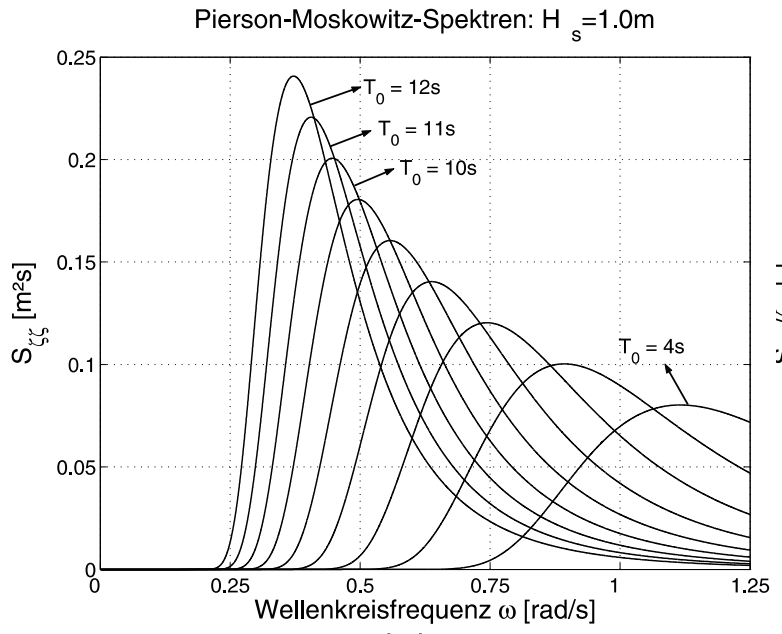

(a)

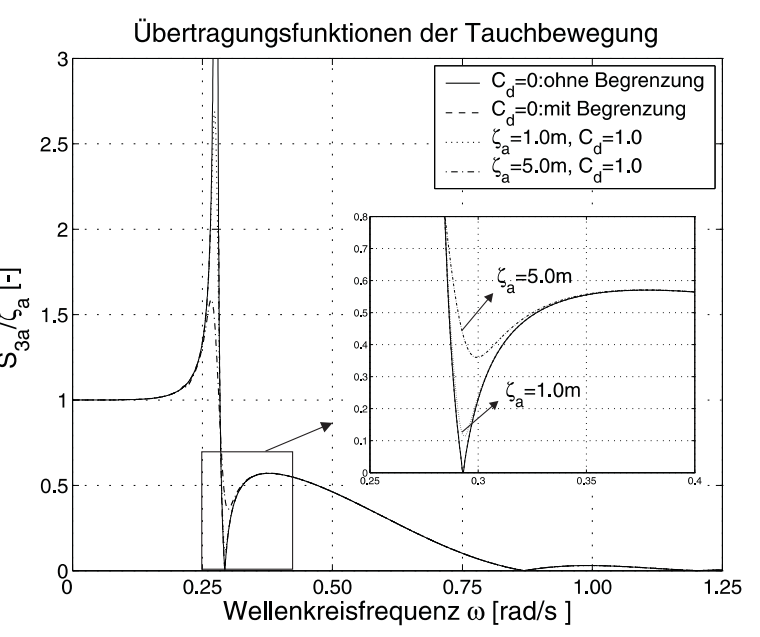

(b)

Abbildung 6.17: Effekt der Zähigkeit auf die Übertragungsfunktion der Tauchbewegung: (a) Pierson-Moskowitz Spektren für $H_{s}=1.0 \mathrm{~m}$ und $T_{0}=[12 \mathrm{~s}, 11 \mathrm{~s}, \cdots, 5 \mathrm{~s}, 4 \mathrm{~s}]$ (b) Übertragungsfunktionen der Tauchbewegung

he Anhang B). Da die mit dem TFC-Programm berechneten Übertragungsfunktionen von der Wellenamplitude $\zeta_{a}$ abhängig sind (siehe Abschnitt 5.2), müssen zuerst die Effekte der Zähigkeit auf die Übertragungsfunktionen der Tauchbewegung mit unterschiedlichen Wellenamplituden untersucht werden. Dann können die Effekte der von der Wellenamplitude abhängigen Zähigkeitsdämpfungen und -kräfte auf das Anwortspektrum der Tauchbewegung untersucht werden, die auch schließlich den Zielfunktionswert, d.h. die zu erwartende Ausfallzeit beeinflussen.

Als Seegangsspektrum wird hier Pierson-Moskowitz Spektrum verwendet. Um die Zähigkeitseffekte auf die Strukturantwort in unterschiedlichen Seegängen zu untersuchen, werden für die Seegangsspektren die signifikante Wellenhöhe $H_{s}=1.0 \mathrm{~m}$ und Zero-up-crossing Perioden $T_{0}=[12 s, 11 s, \cdots, 5 s, 4 s]$ definiert (Abb. 6.17 (a)). Abb. 6.17 (b) zeigt die Übertragungsfunktionen (RAO) der Tauchbewegung für einen Halbtaucher mit Verdrängung von 30,000 $\mathrm{m}^{3}$ (siehe Ausgangsstruktur (A) in Abb.6.1), wobei die Übertragungsfunktionen für die Wellenamplituden $\zeta_{a}=1 \mathrm{~m}$ und $5.0 \mathrm{~m}$ berechnet werden. Zum Vergleich wird auch die Übertragungsfunktion ohne Zähigkeit $\left(C_{d}=0\right)$ dargestellt, bei der der unendliche Resonanzspitzenwert auf eine endliche Amplitude $2[\mathrm{~m} / \mathrm{m}]$ begrenzt wurde.

In Abb. 6.17 (b) zeigt sich deutlich, dass die zähigkeitsbedingte Dämpfung und Kraft nur im Bereich der Resonanz- und der Auslöschungsfrequenz von Bedeutung sind. Bei der Resonanzfrequenz $\omega_{R} \approx 0.27[\mathrm{rad} / \mathrm{s}]$ liegt der Betrag der Übertragungsfunktion für die Tauchbewegung für die Wellenamplitude $\zeta_{a}=1.0 \mathrm{~m}$ etwa bei $2.7[\mathrm{~m} / \mathrm{m}]$, was größer als die auf $\left(s_{3 a} / \zeta_{a}\right)_{\max }=2[\mathrm{~m} / \mathrm{m}]$ begrenzte Übertragungsfunktion ist. Für die Wellenamplitude $\zeta_{a}=5.0 \mathrm{~m}$ begrenzt die zähigkeitsbedingte Dämpfung die Übertragungsfunktion der Tauchbewegung auf $1.55[\mathrm{~m} / \mathrm{m}]$, d.h. je größer die Wellenamplitude $\zeta_{a}$ ist, desto stärker wirkt sich die Zähigkeitsdämpfung auf die Tauchbewegung aus. 

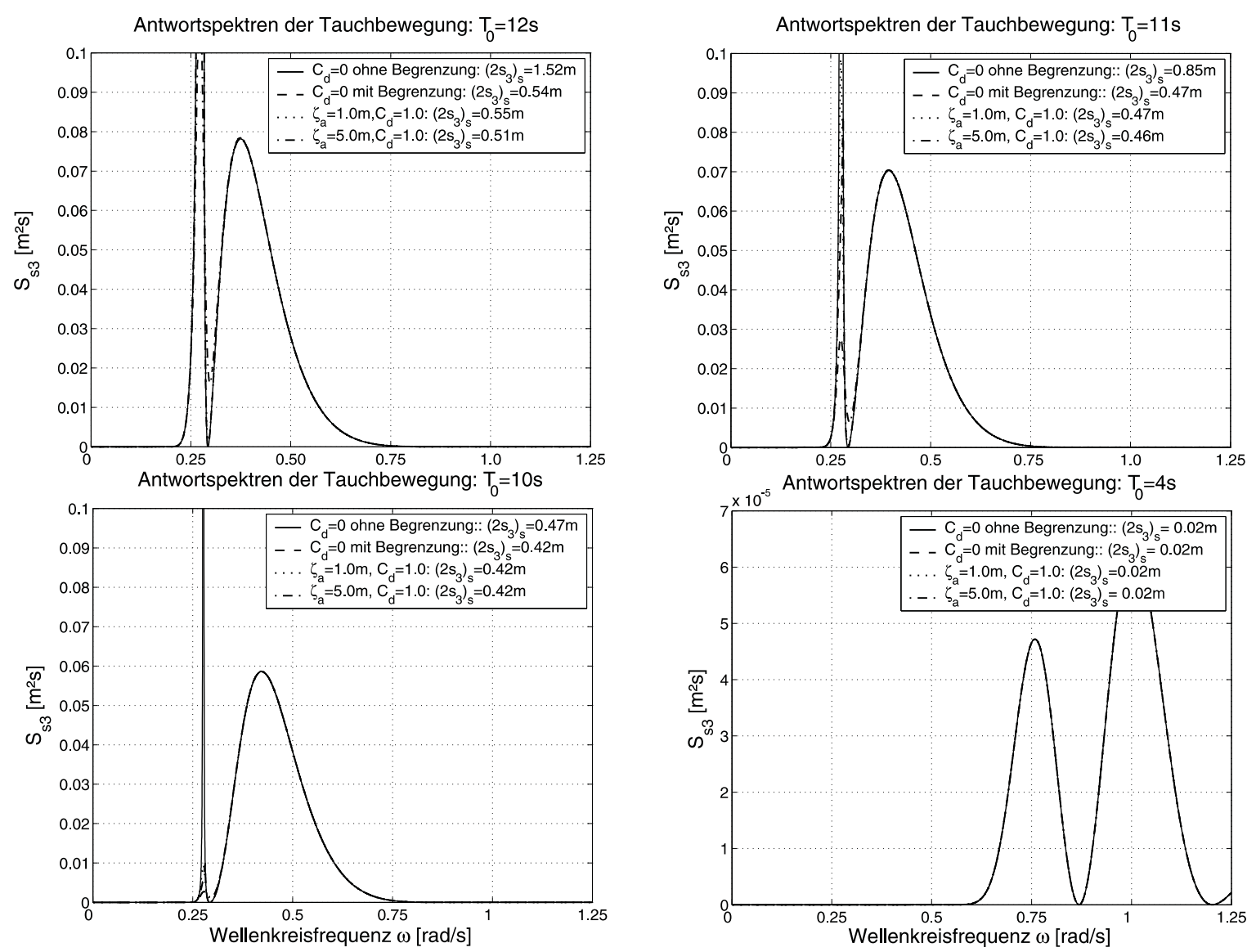

Abbildung 6.18: Effekt der Zähigkeit auf die Antwortspektren der Tauchbewegung für unterschiedliche Zero-up-crossing Perioden $T_{0}$

Im Bereich der Auslöschungsfrequenz $\omega_{C} \approx 0.3[\mathrm{rad} / \mathrm{s}]$ führt die zähigkeitsbedingte Kraft zu endlichen Bewegungen (siehe vergrößertes Bild in Abb. 6.17 (b)). Bei anderen Frequenzen beeinflusst die Zähigkeit die Übertragungsfunktionen der Tauchbewegung nicht merklich.

Nach Gl. 5.11 erhält man die Antwortspektren der Tauchbewegung, indem man die Seegangsspektren in Abb. 6.17 (a) mit dem Quadrat der Übertragungsfunktion in Abb. 6.17 (b) multipliziert. Die Antwortspektren für unterschiedliche Zero-up-crossing Perioden $T_{0}$ in Abb 6.18 zeigen, dass der Effekt der Zähigkeit auf die Strukturantwort auch von dem entsprechenden Seegangszustand abhängt: Für die Zero-up-crossing Perioden $T_{0}=4 s$ sind die signifikanten Doppelamplituden der Tauchbewegung unabhängig von der Wellenamplitude identisch mit $\left(2 s_{3}\right)_{a}=0.02 m$, obwohl die Übertragungsfunktionen der Tauchbewegung abhängig von der Wellenamplitude verschieden sind. Das liegt daran, dass das Seegangsspektrum für $T_{0}=4 s$ im Bereich der Frequenz $\omega<0.5$, in dem Resonanz- und Auslöschungsfrequenz liegen, kaum Seegangsenergie enthält. Im Gegensatz dazu sind bei Antwortspektren für $T_{0}=12 s, 11 s$ die Effekte der Zähigkeit deutlich zu merken: Bei der Resonanzfrequenz wird die Strukturantwort aufgrund der Zähigkeit gedämpft. Bei der Auslöschungsfrequenz führt die Zähigkeitskraft zu 

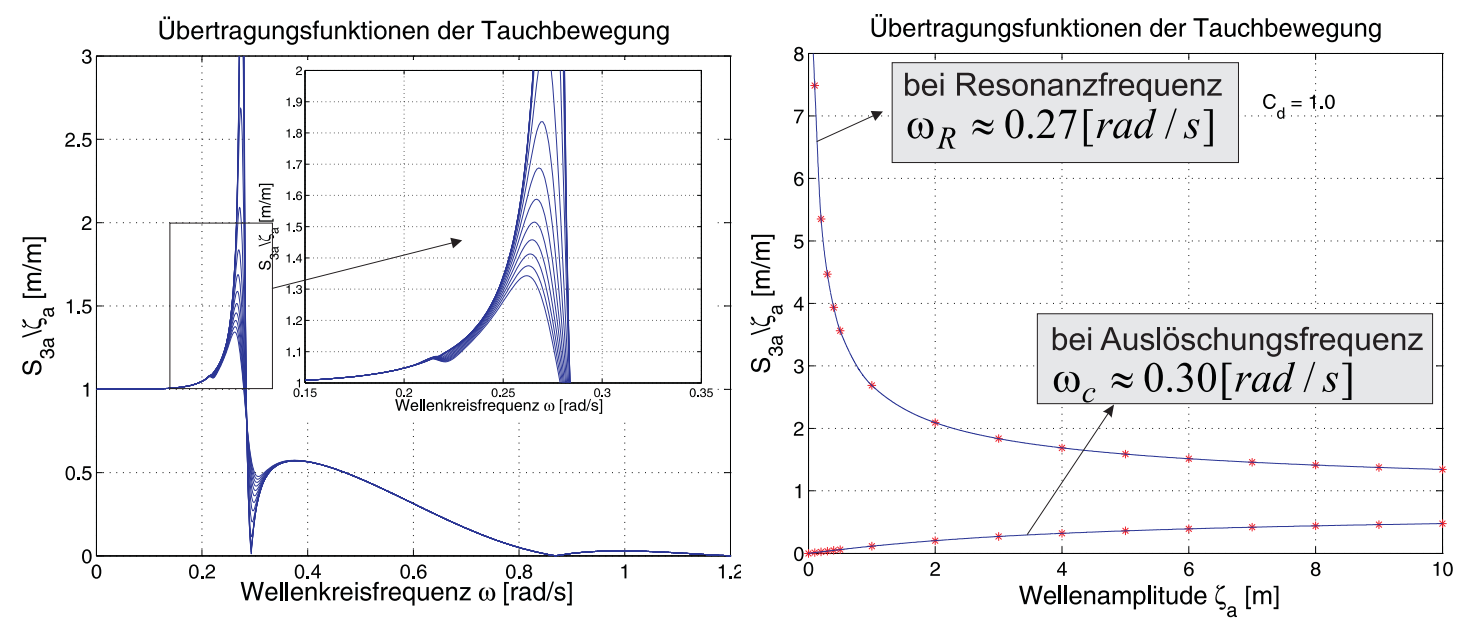

Abbildung 6.19: Einfluss der Zähigkeit auf die Übertragungsfunktion der Tauchbewegung in Abhängigkeit der Wellenamplitude $\zeta_{a}$ bei Resonanz- und Auslöschungsfrequenz

endlichen Strukturbewegungen. Der Einfluss der Zähigkeit auf die Strukturbewegungen ist demnach nur in extremen Seegänge mit $T_{0}>9 s$ von Bedeutung. Außerdem liegt die Resonanzfrequenz des Halbtauchers meistens im Bereich der Wellenfrequenz $0.25<\omega<0.4$, wo der Seegang mit $T_{0} \leq 9 \mathrm{~s}$ in diesem Frequenzbereich wenig Energie besitzt.

Die Abhängigkeit der Übertragungsfunktionen von der Wellenamplitude $\zeta_{a}$ lässt sich deutlich durch Abb. 6.19 zeigen, wobei die Amplitude der Übertragungsfunktionen bei der Resonanzfrequenz und bei der Auslöschungsfrequenz über $\zeta_{a}$ aufgetragen werden. Es wird deutlich, dass sowohl die gedämpften Bewegungen bei der Resonanzfrequenz als auch die endlichen Bewegungen aufgrund der Zähigkeitskraft bei der Auslöschungsfrequenz nicht proportional zu der Wellenamplitude $\zeta_{a}$ sind. Mit zunehmender Wellenamplitude wird das Dekrement der Resonanzpeaks kleiner, wobei auch die Zunahme der endlichen Bewegungsamplitude bei $\omega \approx \omega_{c}$ kleiner wird.

\subsubsection{Zähigkeitseinfluss auf die Optimierung}

Bei der numerischen Berechnung für die Zielfunktion wurden bisher die Resonanzpeaks entweder ohne Begrenzung behandelt oder mit der vordefinierten maximalen Resonanzamplitude (z.B. $s_{3 a} / \zeta_{a}=2$ ) begrenzt. In realistischen Seegangssituationen werden die Starrkörperbewegungen der schwimmenden Strukturen bei Resonanzstellen durch Dämpfungen begrenzt. Um den Einfluss der Dämpfung auf den Optimierungsablauf zu untersuchen, werden die zähigkeitsbedingten Kräfte und Dämpfungen in der hydrodynamischen Formoptimierung berücksichtigt. Als Anwendungsbeispiel für die Optimierung wird hier weiter derselbe Halbtaucher mit 9 freien Variablen verwendet.

Der für die Berechnung der Zähigkeitsdämpfung und -kräfte notwendige Widerstandsbeiwert $C_{d}$ hängt von mehreren Faktoren ab, vor allem von der Reynoldszahl 


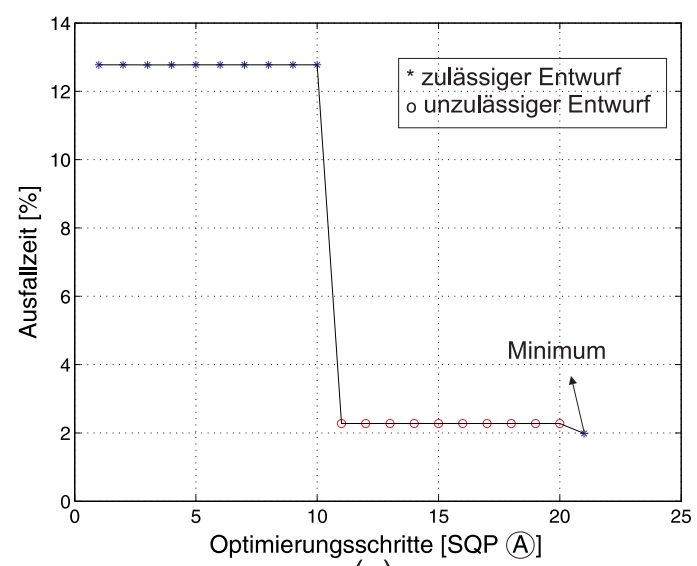

(a)

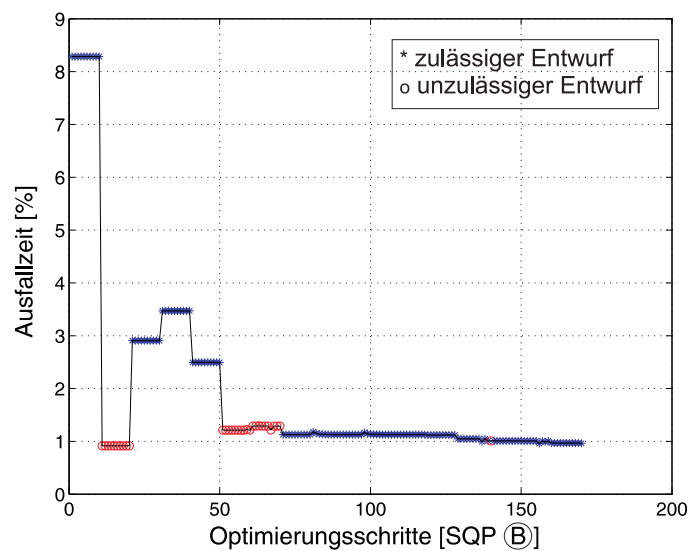

(b)

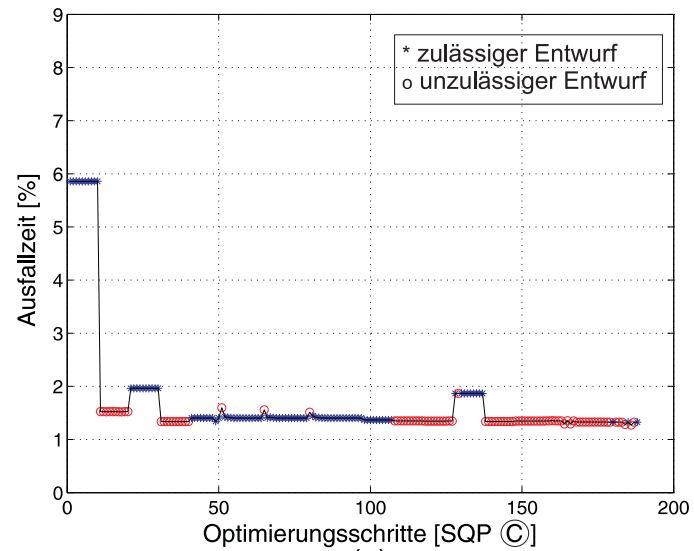

(c)

Abbildung 6.20: Optimierungsschritte und Änderung der Zielfunktion für den SQPAlgorithmus unter Berücksichtigung der Zähigkeit $C_{d}=1.0, \zeta_{a}=1.0 \mathrm{~m}$ : (a) mit der Ausgangsstruktur (A) (b) mit der Ausgangsstruktur (B) (c) mit der Ausgangsstruktur (C)

$\left(R e=u_{a} D / \nu\right)^{1}$ und der Keulegan-Carpenter Zahl $\left(K C=u_{a} T / D\right)^{2}$. Bei der Analyse meerestechnischer Konstruktionen werden diese Beiwerte im allgemeinen durch Messungen gewonnen. Da der $C_{d^{-}}$Beiwert bei der Optimierung nicht automatisch bestimmt werden kann, wird hier der konstante $C_{d}$-Beiwert von 1.0 eingesetzt. Wie bereits in Abschnitt 6.1.3 diskutiert, sind die mit dem TFC-Programm berechneten Übertragungsfunktionen von der Wellenamplitude abhängig. Um den Einfluss der Dämpfung auf die Optimierung systematisch zu untersuchen, wird hier die Optimierung auch für die Wellenamplituden $\zeta_{a}=1.0 \mathrm{~m}$ und $5.0 \mathrm{~m}$ durchgeführt.

Wie der Verlauf der Optimierungen in Abb.6.20 zeigen, weist das SQP-Verfahren bei Berücksichtigung des Zähigkeitseinflusses $\left(C_{d}=1.0, \zeta_{a}=1.0 \mathrm{~m}\right)$ ein anderes Optimierungsverhalten auf (Vergleiche Abb. 6.11). Mit der Ausgangsstruktur (A) bleibt der SQP-Algorithmus nach 20 Schritten in einem lokalen Minimum mit einer Aus-

\footnotetext{
${ }^{1}$ Verhältnis von Trägheitskraft zu Zähigkeitskraft

${ }^{2}$ Verhältnis von Umfang der Wellenpartikelbahn in Tiefwasser zum Bauwerksdurchmesser
} 


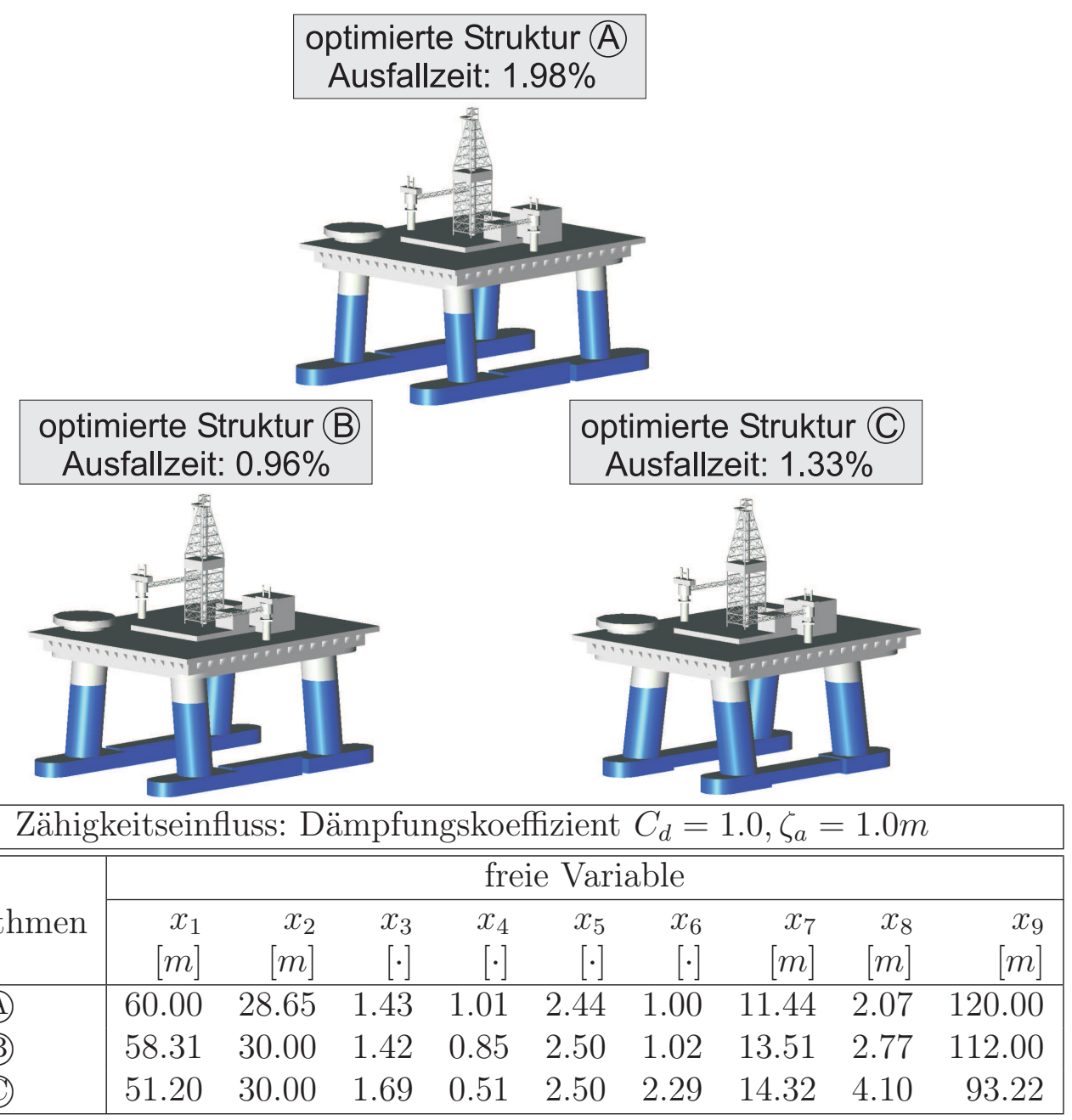

Abbildung 6.21: Geometrie der optimierten Strukturen mit dem lokalen Optimierungsalgorithmus SQP unter Berücksichtigung der Zähigkeit $C_{d}=1.0, \zeta_{a}=1.0 \mathrm{~m}$

fallzeit von $1.98 \%$ stecken. Dagegen erzielt der SQP-Algorithmus ausgehend von der Ausgangsstruktur (B) ein Minimum mit der Ausfallzeit 0.96\%. Auffällig ist dabei, dass die Ausfallzeit der optimierten Struktur SQP (C) mit 1.33\% schlechter ist als die von SQP (B) (Abb. 6.21), während bei Begrenzung der maximalen Amplitude auf $2[\mathrm{~m} / \mathrm{m}]$ der SQP-Algorithmus mit der Ausgangsstruktur (C) ein besseres Minimum erzielen konnte (siehe Abb.6.10). Im Vergleich zu den Geometrien der optimierten Strukturen in Tab. 6.3, zeigen die optimierten Strukturen in Abb. 6.21 deutlich, dass der SQP-Algorithmus unter Berücksichtigung der Zähigkeit leicht unterschiedliche Formparameter findet. In diesem Fall ergeben sich für alle optimierten Strukturen z.B. eine erkennbare Querverschiebung der Säule an der Wasseroberfläche.

Geometrien der optimierten Strukturen durch Anwendung globaler Optimierungsalgorithmen sind mit den minimierten Ausfallzeiten in Abb.6.22 dargestellt. Bei Berücksichtigung der Zähigkeit findet der Genetische Algorithmus nur ein lokales Minimum mit einer Ausfallzeit von 1.57\%, während der Adaptive Simulated Annealing Algorithmus wieder das beste Minimum mit einer Ausfallzeit von 0.94\% erzielt. Ver- 


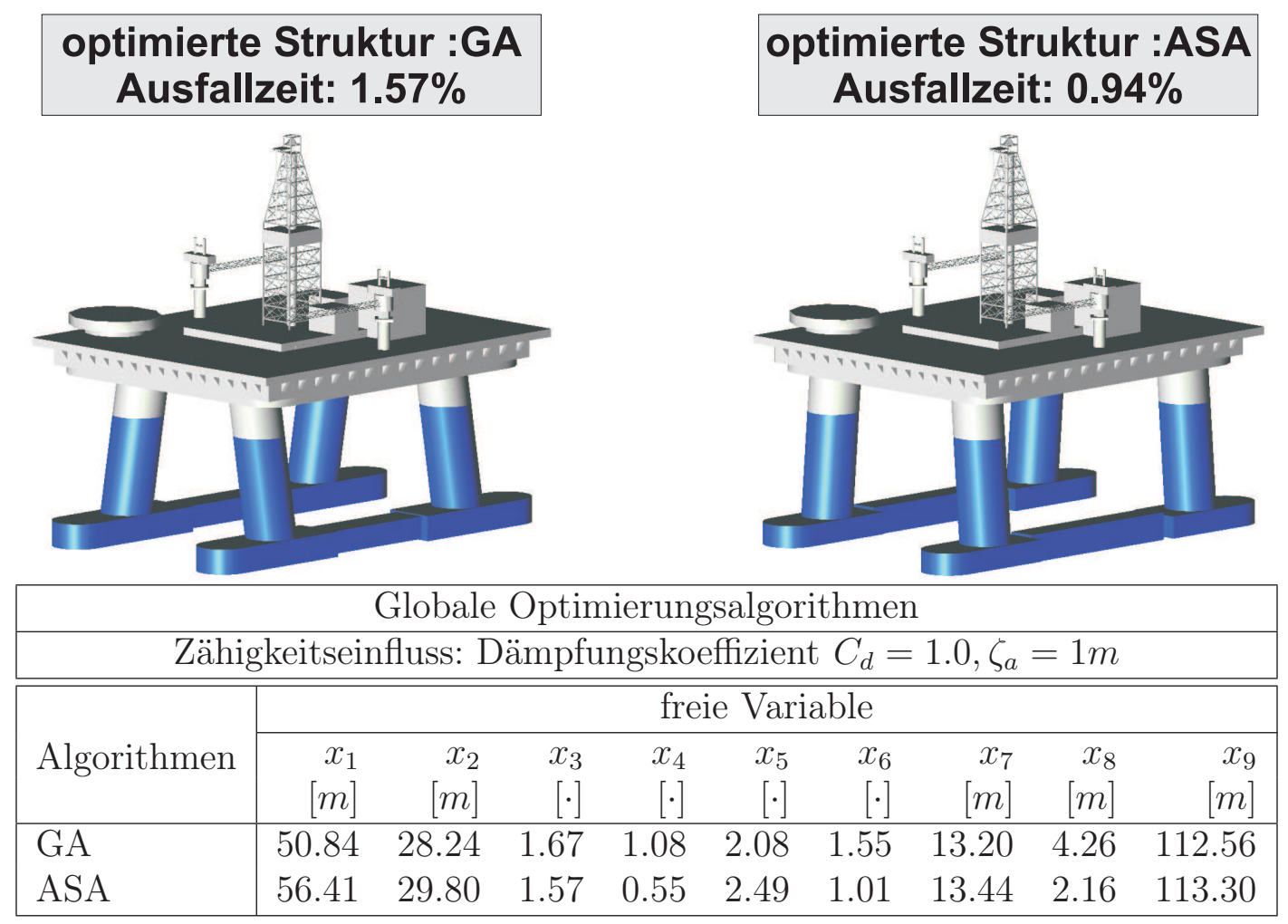

Abbildung 6.22: Geometrie der optimierten Strukturen mit den globalen Optimierungsalgorithmen unter Berücksichtigung der Zähigkeit $C_{d}=1.0, \zeta_{a}=1.0 \mathrm{~m}$

mutlich spielen die zufällig ausgewählten Anfangsgenerationen eine wichtige Rolle für den Erfolg des Genetischen Algorithmus. Durch eine nachgeschaltete Optimierung mit der lokalen Suchstrategie SQP konnte wiederum eine weitere Reduktion der Zielfunktionswerte erzielt werden (Abb.6.23). Der lokale SQP-Algorithmus kann also mit mehrfachen Startpunkten oder durch gezielte Kombination stochastischer Methoden auch global sein und für multimodale Zielfunktionen eingesetzt werden. Das beste Minimum mit der Ausfallzeit von $0.85 \%$ wurde hierbei aus der durch ASA minimierten Struktur gewonnen. Das gefundene Minimum der optimierten Struktur von GA+SQP ist jedoch mit einer Ausfallzeit von 1.16\% nicht besser als das durch SQP (B) erzielte. Trotz der Unterschiede in den Hauptabmessungen und in den Zielfunktionswerten weisen alle optimierte Strukturen in Abb 6.22 und in Abb 6.23 auch eine ähnliche Formänderungstendenz auf, d.h. die Vergrößerung des Tiefgangs und die Verteilung der Pontonverdrängung von der Pontonmitte zu den Pontonenden wie schon bei den optimierten Strukturen in Abb.6.14 und in Abb.6.15.

Die Anzahl der benötigten Zielfunktionsbewertungen und die zu erwartenden Ausfallzeiten sind nach den Optimierungsalgorithmen in Tab. 6.6 zusammengefasst. Dabei werden die Optimierungsergebnisse abhängig von den verwendeten Wellenamplituden $\zeta_{a}=1.0 \mathrm{~m}$ und $\zeta_{a}=5.0 \mathrm{~m}$ verglichen. Die Zähigkeitsdämpfung und -kräfte beeinflussen sowohl den Optimierungsablauf als auch die Zielfunktionswerte (vergleiche Tab 6.4). Vor allem ist der Zähigkeitseinfluss auf das Optimierungsverhalten bei der lokalen Suchstrategie SQP deutlich. Bei Berücksichtigung der Zähigkeit mit $\zeta_{a}=5.0 \mathrm{~m}$ finden die 

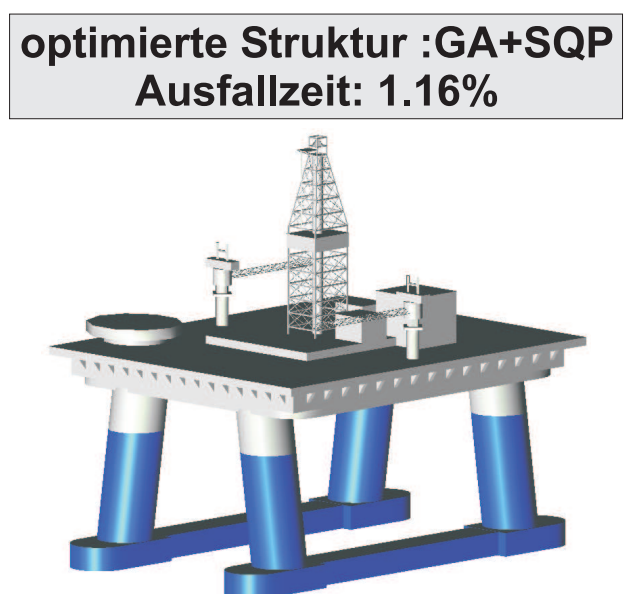
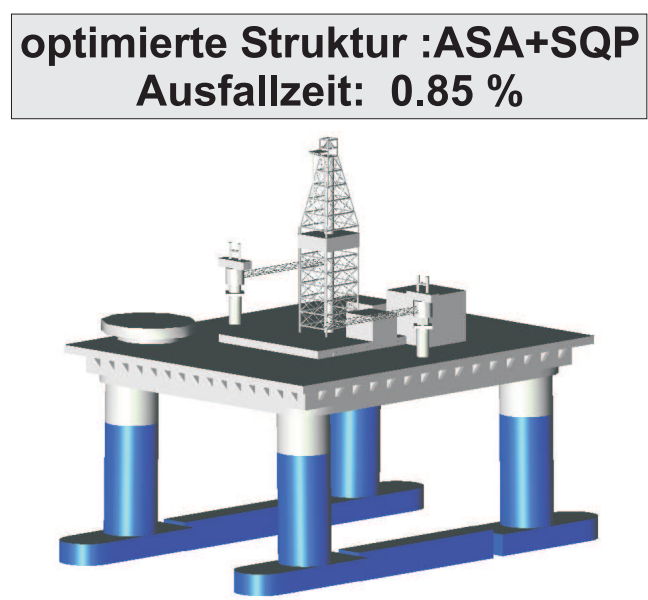

\begin{tabular}{|l|rrrrrrrrr|}
\hline \multicolumn{10}{|c|}{ Hybride Optimierungsalgorithmen } \\
\hline \multicolumn{10}{|c|}{ Zähigkeitseinfluss: Dämpfungskoeffizient $C_{d}=1.0, \zeta_{a}=1 m$} \\
\hline \hline \multirow{3}{*}{ Algorithmen } & \multicolumn{10}{c|}{ freie Variable } \\
\cline { 2 - 10 } & $x_{1}$ & $x_{2}$ & $x_{3}$ & $x_{4}$ & $x_{5}$ & $x_{6}$ & $x_{7}$ & $x_{8}$ & $x_{9}$ \\
& {$[m]$} & {$[m]$} & {$[\cdot]$} & {$[\cdot]$} & {$[\cdot]$} & {$[\cdot]$} & {$[m]$} & {$[m]$} & {$[m]$} \\
\hline GA + SQP & 53.54 & 30.00 & 1.72 & 0.35 & 2.38 & 1.62 & 13.53 & 3.37 & 111.04 \\
ASA + SQP & 60.00 & 30.00 & 1.42 & 0.65 & 2.50 & 1.00 & 13.34 & 0.39 & 120.00 \\
\hline
\end{tabular}

Abbildung 6.23: Geometrie der optimierten Strukturen mit den hybriden Optimierungsalgorithmen unter Berücksichtigung der Zähigkeit $C_{d}=1.0, \zeta_{a}=1.0 \mathrm{~m}$

\begin{tabular}{|c|c|c|c|c|}
\hline \multicolumn{5}{|c|}{ Zähigkeitseinfluss: Dämpfungskoeffizient $C_{d}=1.0$} \\
\hline \hline Algorithmen & \multicolumn{2}{|l|}{$\begin{array}{l}\text { Anzahl der Bewertungen } \\
\text { der Zielfunktion }\end{array}$} & $\begin{array}{l}\text { Ausfallzeit } \\
\text { in } \%\end{array}$ \\
\cline { 2 - 5 } & $\zeta_{a}=1.0 m$ & $\zeta_{a}=5.0 m$ & $\zeta_{a}=1.0 m$ & $\zeta_{a}=5.0 m$ \\
\hline SQP (A) & 21 & 186 & 1.98 & 1.25 \\
SQP B & 170 & 31 & 0.96 & 2.37 \\
SQP C) & 187 & 72 & 1.33 & 0.85 \\
GA & 1321 & 1284 & 1.57 & 1.40 \\
GA + SQP & $1321+197$ & $1284+89$ & 1.16 & 1.01 \\
ASA & 2588 & 730 & 0.94 & 1.32 \\
ASA + SQP & $2588+96$ & $730+121$ & 0.85 & 0.77 \\
\hline
\end{tabular}

Tabelle 6.6: Vergleich der Optimierungsergebnisse bei Berücksichtigung des Zähigkeitseinflusses 
globalen Algorithmen (GA und ASA) nur lokale Minima, da das nachgeschaltete lokale SQP Verfahren z.B. mit der Ausgangsstruktur (C) ein besseres Minimum der Ausfallzeit von $0.85 \%$ lokalisieren konnte. ASA benötigt hierbei vergleichsweise weniger Zielfunktionsbewertungen als der Genetische Algorithmus. Die nachgeschaltete Optimierung mit dem SQP Verfahren ermöglicht ebenfalls eine weitere Reduktion der Zielfunktionswerte, wobei das beste Minimum der Ausfallzeit von $0.77 \%$ ausgehend von der durch ASA optimierten Struktur (1.32\%) erzielt wurde.

Die Verbesserung der hydrodynamischen Eigenschaften lässt sich deutlich anhand der Übertragungsfunktionen zeigen (Abb. 6.24). Im Bereich der mittleren Wellenfrequenzen mit $0.4<\omega<0.7$ ist festzustellen, dass die durch die Formoptimierung durchgeführten Änderungen an der Geometrie die Bewegungsamplituden um mehr als die Hälfte verringern. Obwohl die hydrodynamischen Formoptimierungen mit Hilfe der Begrenzung der maximalen Übertragungsfunktion auf $2[\mathrm{~m} / \mathrm{m}]$ erfolgreich durchgeführt wurden (siehe Abschnitt 6.1.2), sind die Unterschiede in den Übertragungsfunktionen der Tauchbewegung aufgrund der Zähigkeiteffekte deutlich vorhanden.

Wie Abb. 6.24 (c) zeigt, spielt die Zähigkeitsdämpfung besonders im Bereich der Tauchresonanz bei $\omega_{R} \approx 0.3[\mathrm{rad} / \mathrm{s}]$ eine Rolle. Der von der Wellenamplitude abhängige Dämpfungskoeffizient begrenzt die Bewegungsamplitude auf endliche Werte. Bei der Auslöschungsfrequenz $\omega_{C} \approx 0.35[\mathrm{rad} / \mathrm{s}]$ verschwindet die Trägheitskraft, so dass die Wellenkraft auf die Struktur ausschließlich aus der geschwindigkeitsabhängigen Zähigkeitskraft besteht. Die Widerstandskraft führt zu endlichen Tauchbewegungen im Bereich der Auslöschungsfrequenz. Im Gegensatz zeigt Abb 6.24 (a), dass die Bewegungsamplituden bei Auslöschungsfrequenz gleich Null sind (ohne Berücksichtigung der Zähigkeit), wobei die Resonanzpeaks einheitlich auf $2[\mathrm{~m} / \mathrm{m}]$ beschränkt wurden. Trotz dieser Unterschiede wurden die Höhe der Zielfunktionswerte für die optimierten Strukturen von der Zähigkeit nicht wesentlich beeinflusst. Dies liegt daran, dass für das betrachtete Seegebiet Haltenbanken nur kleine Änderungen der unzulässigen Seegangszustände im Bereich der Zero-Up-Crossing Perioden mit $T_{0}>15 \mathrm{~s}$ auftreten (siehe Abb.6.26).

Als Folge der Formoptimierung erhöht sich jedoch die Resonanzfrequenz der Tauchbewegung (siehe Abb 6.24). Dies führt besonders im extremen Seegang zu großen Strukturbewegungen. Um die Konsequenz der Verschiebung der Resonanzfrequenz bei optimierten Strukturen zu untersuchen, werden die Antwortspektren der optimierten Strukturen für ein Seegangsspektrum mit $H_{s}=10 \mathrm{~m}$ und $T_{0}=12 \mathrm{~s}$ berechnet. Für das betrachtete Seegebiet Haltenbanken tritt ein solcher extremer Seegangszustand auf, auch wenn die statistische Häufigkeit gering ist (siehe Abb.5.6). Wie Abb.6.25 zeigt, ist die signifikante Doppelamplitude für die mit der Wellenamplitude $\zeta_{a}=1.0 \mathrm{~m}$ optimierte Struktur ist mit $\left(2 s_{3 a}\right)_{s}=7.01[\mathrm{~m}]$ größer als bei der Ausgangsstruktur mit $\left(2 s_{3 a}\right)_{s}=4.65[\mathrm{~m}]$. Für die mit $\zeta_{a}=5.0 \mathrm{~m}$ optimierte Struktur ist die Strukturantwort mit $\left(2 s_{3 a}\right)_{s}=5.17[\mathrm{~m}]$ ebenfalls größer. Wie der Gl.5.14 folgt, ist die größere signifikante Doppelamplitude der Tauchbewegung für die optimierten Strukturen der Grund für die kleinere zulässige signifikante Wellenhöhe im Bereich der Zero-up-crossing Periode mit $T_{0}>10 s$ (siehe Abb.6.26).

In Abb. 6.26 werden die Ergebnisse von dem lokalen Algorithmus SQP mit der 


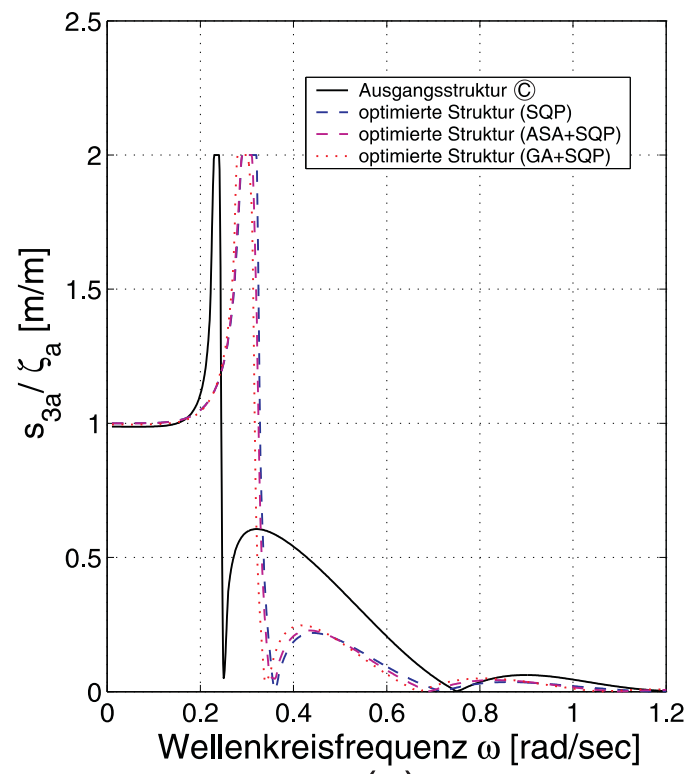

(a)

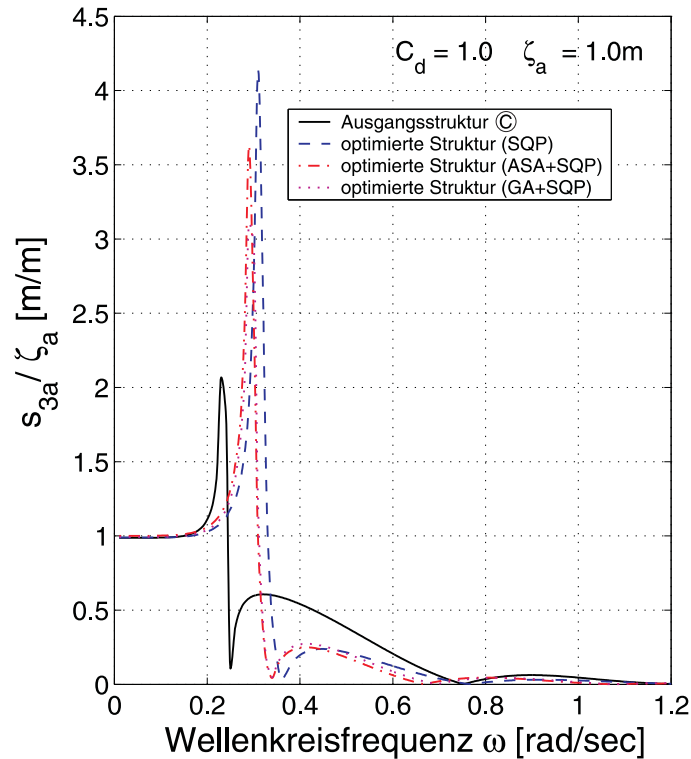

(b)

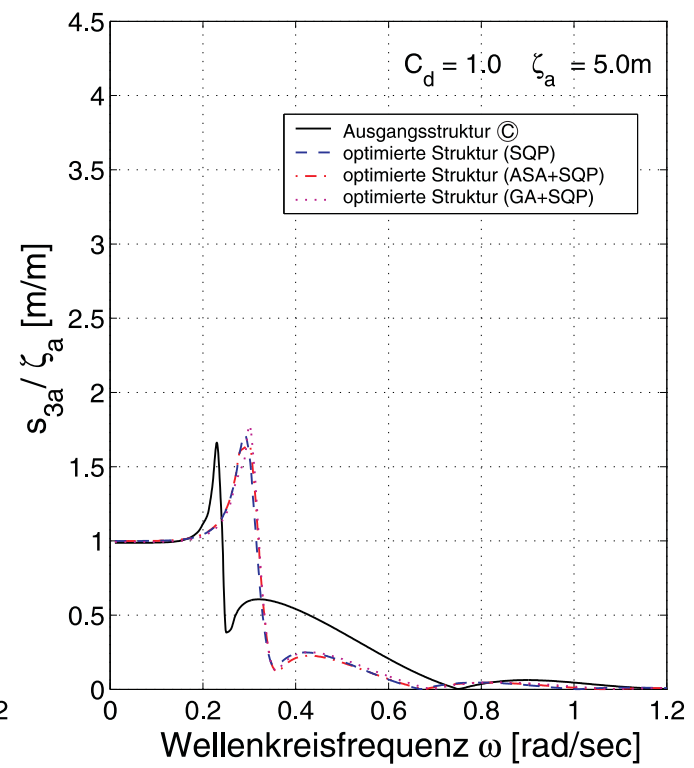

(c)

Abbildung 6.24: Vergleich der Übertragungsfunktionen der Tauchbewegung: Optimierung (a) mit Begrenzung des maximalen RAOs auf $2[\mathrm{~m} / \mathrm{m}]$ (b) mit Berücksichtigung des Zähigkeitseinflusses mit $C_{d}=1.0, \zeta_{a}=1.0 \mathrm{~m}$ (c) mit Berücksichtigung des Zähigkeitseinflusses mit $C_{d}=1.0, \zeta_{a}=5.0 \mathrm{~m}$ 


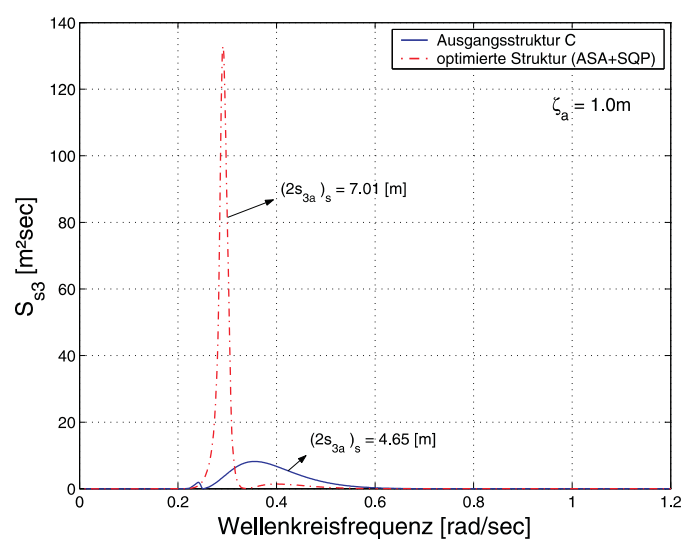

(a)

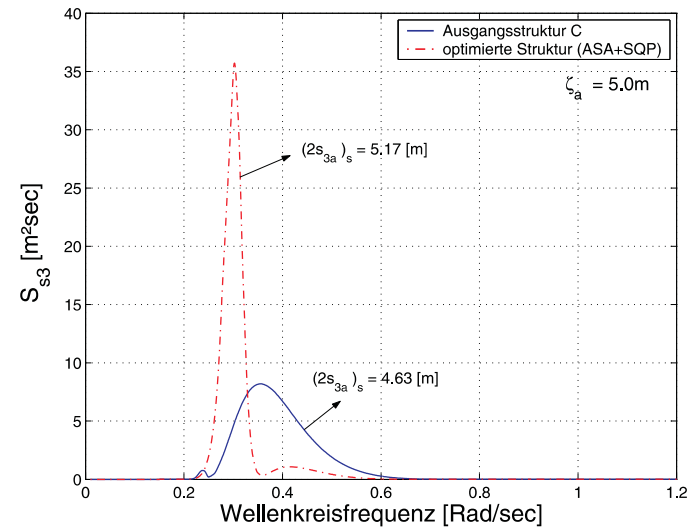

(b)

Abbildung 6.25: Vergleich der Antwortspektren der Tauchbewegung für die optimierten Strukturen (ASA+SQP): (a) mit Berücksichtigung des Zähigkeitseinflusses mit $C_{d}=$ $1.0, \zeta_{a}=1.0 \mathrm{~m}$ (b) mit Berücksichtigung des Zähigkeitseinflusses mit $C_{d}=1.0, \zeta_{a}=$ $5.0 \mathrm{~m}$
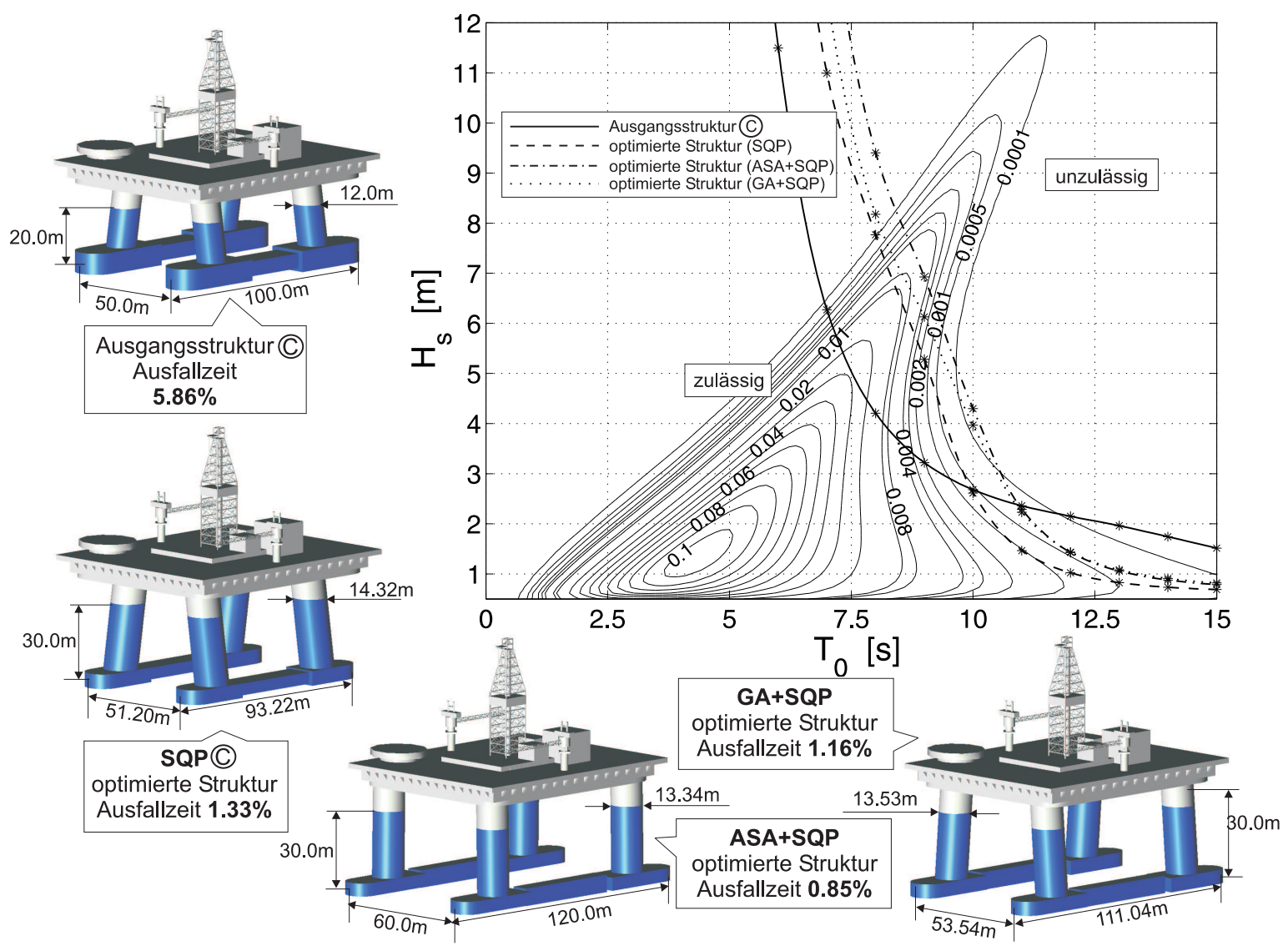

Abbildung 6.26: Vergleich der Geometrien und Grenzkurven der zulässigen signifikanten Wellenhöhen: Optimierungen mit Ausgangsstruktur (C) bei Berücksichtigung der Zähigkeit $C_{d}=1.0, \zeta_{a}=1.0 \mathrm{~m}$ 
Ausgangsstruktur $\mathbb{C}$ und von den globalen Algorithmen (ASA+SQP, GA+SQP) zusammengefasst, wobei die Zähigkeit mit $C_{d}=1.0, \zeta_{a}=1.0 \mathrm{~m}$ berücksichtigt wurde. Während bei Begrenzung der maximalen Bewegungsamplitude auf $2[\mathrm{~m} / \mathrm{m}]$ die Grenzkurven der zulässigen signifikanten Wellenhöhen für die optimierten Strukturen fast identisch sind (Abb. 6.16), ist bei Berücksichtigung der Zähigkeit mit der $\zeta_{a}=1.0 \mathrm{~m}$ eine deutliche Abweichung hydrodynamischer Eigenschaften der optimierten Strukturen vorhanden. Bei Berücksichtigung der Zähigkeit mit $\zeta_{a}=5.0 \mathrm{~m}$ sind die Unterschiede in den Grenzkurven der zulässigen signifikanten Wellenhöhen gering, da die Übertragungsfunktionen der Tauchbewegung für die optimierten Strukturen in Abb 6.24 (c) fast identisch sind. 


\subsection{Optimierung hydrodynamisch kompakter Struk- turen}

Die Analyse hydrodynamisch kompakter Strukturen ist wesentlich aufwendiger als für hydrodynamisch transparente Strukturen. Letztere kann auf einem handelsüblichen PC in wenigen Sekunden ausgeführt werden, während die potentialtheoretische Analyse nach der Diffraktionstheorie selbst auf einer Hochleistungsworkstation noch fünf Minuten dauert. Deshalb wurde für die hydrodynamische Formoptimierung kompakter Strukturen bislang nur eine lokale Suchstrategie eingesetzt. Trotz der guten Ergebnisses mit der Tangentensuchmethode bei vielen Optimierungen (Clauss und Birk, 1994b, 1998b; Birk et al., 2002) bleibt festzustellen, dass Tests mit deutlich mehr freien Variablen und zusätzlichen Nebenbedingungen keinen so deutlichen Optimierungserfolg erbrachten. Im Rahmen dieser Arbeit werden für die Formoptimierung hydrodynamisch kompakter Strukturen die globalen Optimierungsstrategien eingesetzt, um bestmögliche Kombination der Entwurfsparameter zu bestimmen. Zum Vergleich wird dabei auch die lokale Suchstrategie SQP eingesetzt. Der erhöhte Rechenaufwand bei der Anwendung globaler Algorithmen ist unvermeidlich, doch Dank der rapiden Entwicklung der PC-Leistung können Formoptimierungen in einer Woche ausgeführt werden. Der Vergleich der Optimierungsstrategien wird mittels Formoptimierung zweier typischen hydrodynamisch kompakten Strukturen durchgeführt:

- Halbtaucher großer Verdrängung

- Tension-Leg Plattformen.

\subsubsection{Halbtaucher}

Als hydrodynamisch kompakte Strukturen wird zuerst ein größerer Halbtaucher mit einer Gesamtverdrängung $\triangle=50000 \mathrm{~m}^{3}$ optimiert. Das geplante Einsatzgebiet liegt in diesem Fall im Nordostatlantik. Als Seegangsstatistik werden die nach zwölf Windrichtungen aufgeteilten Tabellen nach Hogben und Lumb (1967, Marsden Square 182) verwendet. Die Zielfunktion in dieser Optimierung ist wiederum die statistisch zu erwartende Ausfallzeit nach (5.14) und (5.16) mit $\left(2 s_{3 a}\right)_{s, z u l}=1.0 \mathrm{~m}$. Wie aus der statistischen Verteilung der Seegangszustände in Abb. 6.27(a) folgt, herrschen vorwiegend südwestliche Winde, d.h. die Wellen breiten sich in nordöstliche Richtung aus. Die häufigste Seegangsrichtung ( $\beta=240 \mathrm{Grad})$ weist auch die größte Häufigkeit an schweren Stürmen auf. Die diskreten Verteilungen werden, wie z.B. in Abb.6.27 (b) gezeigt, durch nichtlineare Regression als kontinuierliche 2D Wahrscheinlichkeitsdichtefunktion dargestellt.

Der zu optimierende Halbtaucher besteht aus vier Säulen und zwei Pontons (Abb. 6.28). Eine Pontonhälfte wurde (wie in Abb.5.3 dargestellt) in vier Abschnitte unterteilt: Mittelsektion, Übergang, Hauptsektion (unter der Säule) und Endsektion. Als freie Variable werden ähnlich wie das Anwengungsbeispiel in Abschnitt 6.1.2 neun Formparameter gewählt:

- der Abstand zwischen beiden Pontonachsen: $x_{1}=P_{\text {sep }}$ 


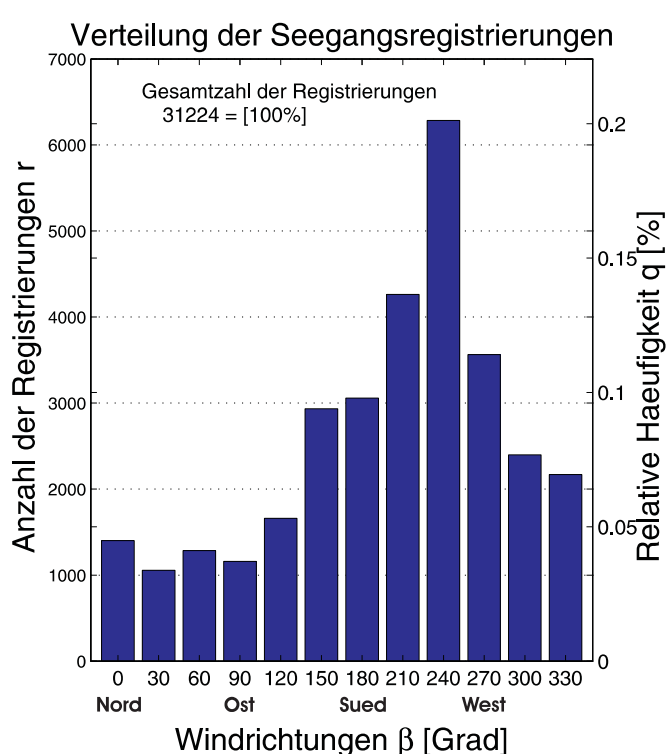

(a)

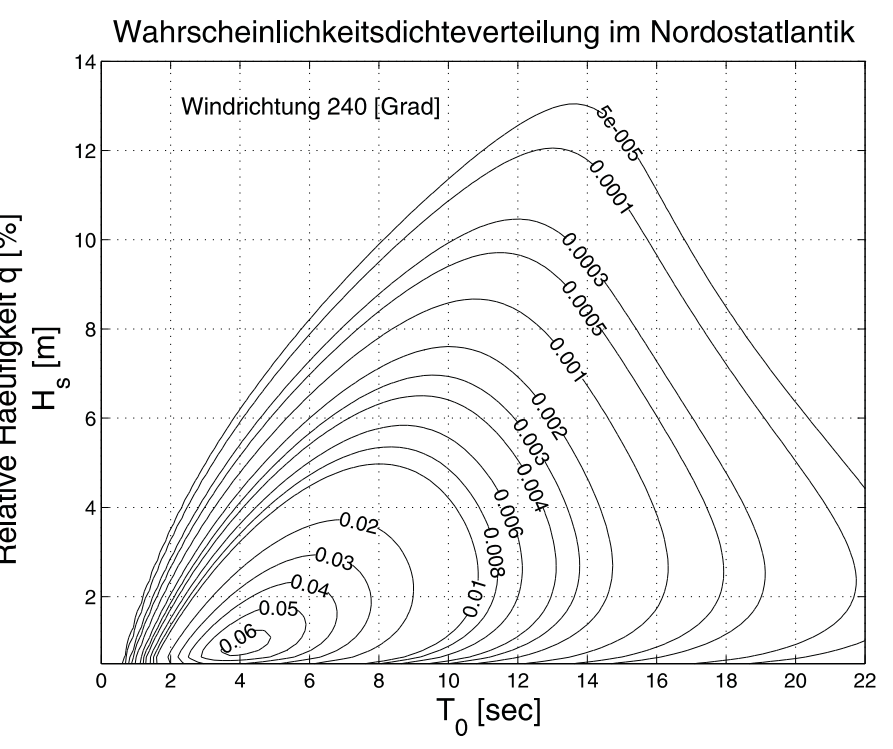

(b)

Abbildung 6.27: (a) Verteilung der Häufigkeit von Seegangszuständen mit Windrichtung $\beta$ im Nordostatlantik (Marsden Square 182) (b) Abgeleitete 2D Wahrscheinlichkeitsdichteverteilung für die Windrichtung 240 [Grad]

- die Tauchtiefe der Pontonachse: $x_{2}=D_{p}$

- das Verhältnis der Querschnittsflächen von Hauptsektion und Mittelsektion: $x_{3}=A_{p m} / A_{p c}$

- das Verhältnis der Länge von Hauptsektion und Mittelsektion: $x_{4}=L_{p m} / L_{p c}$

- das Verhältnis von Breite zu Höhe der Hauptsektion: $x_{5}=B_{p m} / H_{p m}$

- das Verhältnis von Breite zu Höhe der Mittelsektion: $x_{6}=B_{p c} / H_{p c}$

- der Durchmesser der Säulen: $x_{7}=D_{c}$

- die Querverschiebung der Säulen an der Wasseroberfläche (nach innen): $x_{8}=\triangle y_{c}$ und

- die relative Lage des Volumenschwerpunktes für die Säule $V C B / L\left(\right.$ von WL aus): $x_{9}=\xi_{c c}$

Die Länge des Pontons wird allerdings hierbei mit $L_{p}=106 \mathrm{~m}$ als unveränderlicher Formparameter festgelegt. Zusätzlich wird auch die Länge des Übergangspontons mit $L_{p i}=5 \mathrm{~m}$ als konstanter Formparameter eingeführt.

Als Nebenbedingung wird die hydrostatische Anfangsstabilität überprüft. Es wird verlangt, dass die metazentrische Höhe mindestens $1 \mathrm{~m}$ beträgt, d.h. $\overline{G M}>\overline{G M}_{\text {min }}=$ 


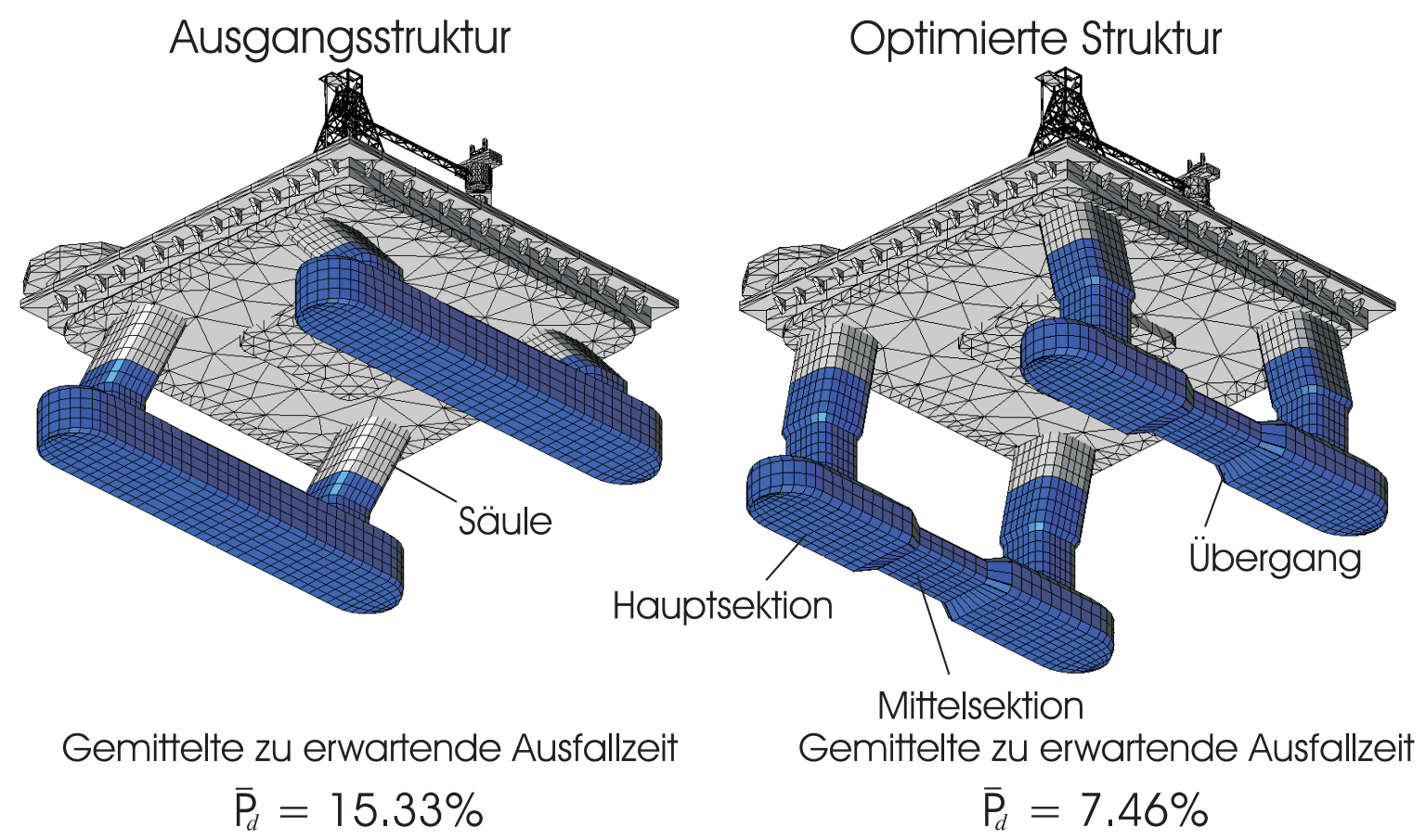

Abbildung 6.28: Ergebnis der Optimierung eines hydrodynamisch kompakten Halbtauchers mit dem Sequential Quadratic Programming Algorithmus (SQP)

$1 m$. Da sich die Säulendurchmesser, der Tiefgang und der Säulenabstand bei der Optimierung ständig ändern, müssen die für die Anfangsstabilität wichtigen Größen (Wasserlinienträgheitsmomente $I_{L}, I_{T}$, Auftriebs- $B$ und Gewichtsschwerpunkt $G$ ) für jeden Entwurf neu ermittelt werden. Die Verschiebung des Gewichtsschwerpunktes wurde hierbei durch ein einfaches Massenmodell in Abhängigkeit vom Auftriebsschwerpunkt abgeschätzt. Die Gesamtverdrängung des Halbtauchers bleibt konstant. Nebenbedingungen hydrostatischer Stabilität, Ober- und Untergrenzen für jede freie Variable begrenzen den Lösungsraum. Um eine Entartung der Geometrie während der Optimierung möglichst auszuschließen, müssen die Grenzen der freien Variablen vor der Optimierung sorgfältig untersucht und damit geometrisch sinnvoll definiert werden. Trotzdem kann es bei der Optimierung vorkommen, dass infolge der zufälligen Kombinationen der Formparameter die Geometrieerzeugung versagt. Für diesen Fall wird der Zielfunktion als Strafe die Ausfallzeit 100\% zugewiesen, um ohne Abbruch den Ablauf der Optimierung zu sichern.

Die minimierten Zielfunktionswerte und die Anzahl der Funktionsauswertungen sind nach den verwendeten Algorithmen in Tab.6.7 zusammengefasst. Wie Abb.6.29 (a) zeigt, minimiert der Sequential Quadratic Programming Algorithmus die gemittelte zu erwartende Ausfallzeit in 184 Zielfunktionsauswertungen. Beim 11-ten und 42-sten Optimierungsschritt wurden die für die hydrodynamische Analyse notwendigen Panelelemente infolge der Entartung der Geometrie nicht erzeugt und deshalb die Ausfallzeit von $100 \%$ als Strafe der Zielfunktionen zugeteilt. SQP verringert die gemittelte zu erwartende Ausfallzeit von $\bar{P}_{d}=15.3 \%$ auf $7.5 \%$.

Mit deutlich größerem Rechenaufwand erzielen die globalen Algorithmen bessere 


\begin{tabular}{|c|c|c|}
\hline Algorithmen & $\begin{array}{l}\text { Anzahl der } \\
\text { Zielfunktionsbewertungen }\end{array}$ & $\begin{array}{l}\text { gemittelte } \\
\text { Ausfallzeit in \% }\end{array}$ \\
\hline \multicolumn{2}{|c|}{ Ausgangsstruktur } & 15.33 \\
\hline SQP & 184 & 7.46 \\
\hline GA & 1338 & 7.35 \\
\hline ASA & 693 & 7.38 \\
\hline
\end{tabular}

Tabelle 6.7: Vergleich der Ergebnisse der hydrodynamischen Formoptimierungen für einen Halbtaucher mit $\triangle=50,000 m^{3}$

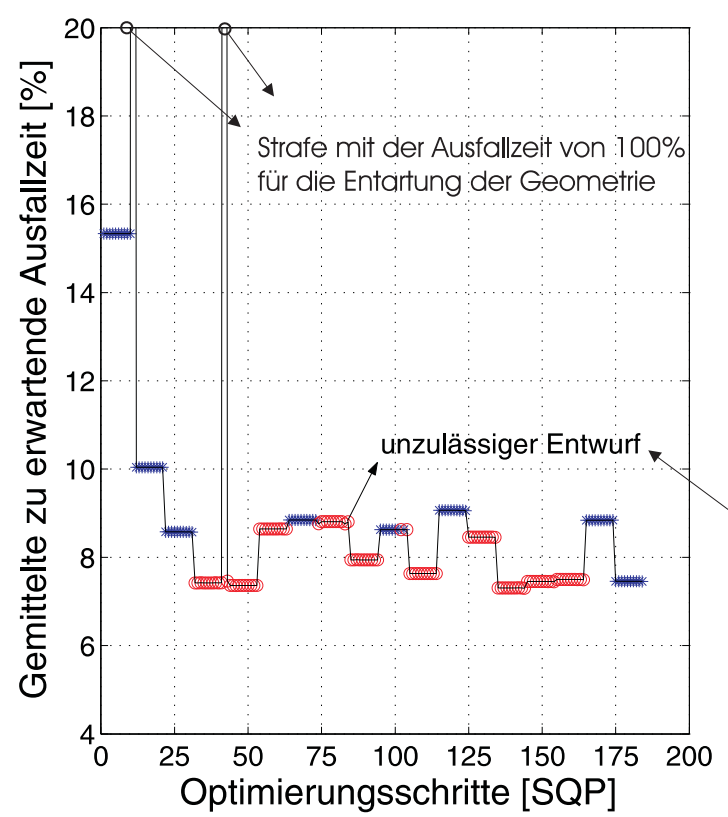

(a)

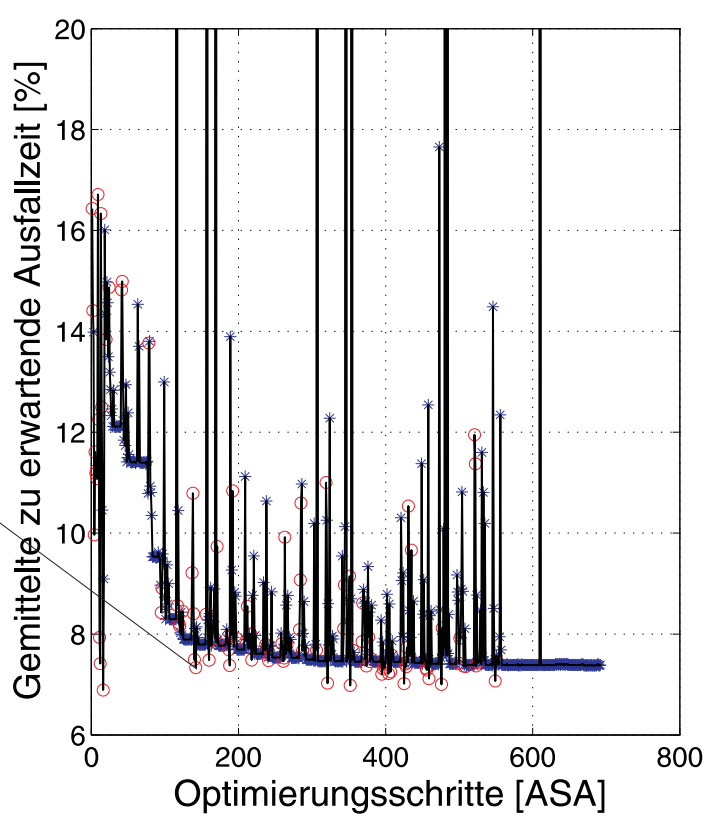

(b)

Abbildung 6.29: Optimierungsschritte und Änderung der Zielfunktion: (a) für den Sequential Quadratic Programming Algorithmus, (b) für den Adaptive Simulated Annealing Algorithmus

\begin{tabular}{|c|c|c|c|c|c|c|c|c|c|}
\hline \multirow[b]{2}{*}{ Algorithmen } & \multicolumn{9}{|c|}{ freie Variable } \\
\hline & $\begin{array}{l}P_{\text {sep }} \\
{[m]}\end{array}$ & $\begin{array}{c}D_{p} \\
{[m]}\end{array}$ & $\begin{array}{c}\frac{A_{p m}}{A_{p c}} \\
{[\cdot]}\end{array}$ & $\begin{array}{c}\frac{L_{p m}}{L_{p c}} \\
{[\cdot]}\end{array}$ & $\begin{array}{c}\frac{B_{p m}}{H_{p m}} \\
{[\cdot]}\end{array}$ & $\begin{array}{c}\frac{B_{p c}}{H_{p c}} \\
{[\cdot]}\end{array}$ & $\begin{array}{l}D_{c} \\
{[m]}\end{array}$ & $\begin{array}{c}\triangle y_{c} \\
{[m]}\end{array}$ & $\begin{array}{c}\xi_{c c} \\
{[\cdot]}\end{array}$ \\
\hline Ausgangsstruktur & 80.0 & 15.0 & 1.00 & 2.00 & 1.50 & 1.50 & 13.50 & 4.00 & 0.50 \\
\hline SQP & 85.0 & 30.0 & 2.11 & 2.65 & 2.12 & 2.37 & 13.84 & 2.61 & 0.47 \\
\hline GA & 79.44 & 29.08 & 1.39 & 1.72 & 1.92 & 1.91 & 13.61 & 4.17 & 0.49 \\
\hline ASA & 82.63 & 29.31 & 2.25 & 1.89 & 2.46 & 2.49 & 14.43 & 5.17 & 0.48 \\
\hline
\end{tabular}

Tabelle 6.8: Freie Variable der Ausgangsstruktur und der optimierten Strukturen 


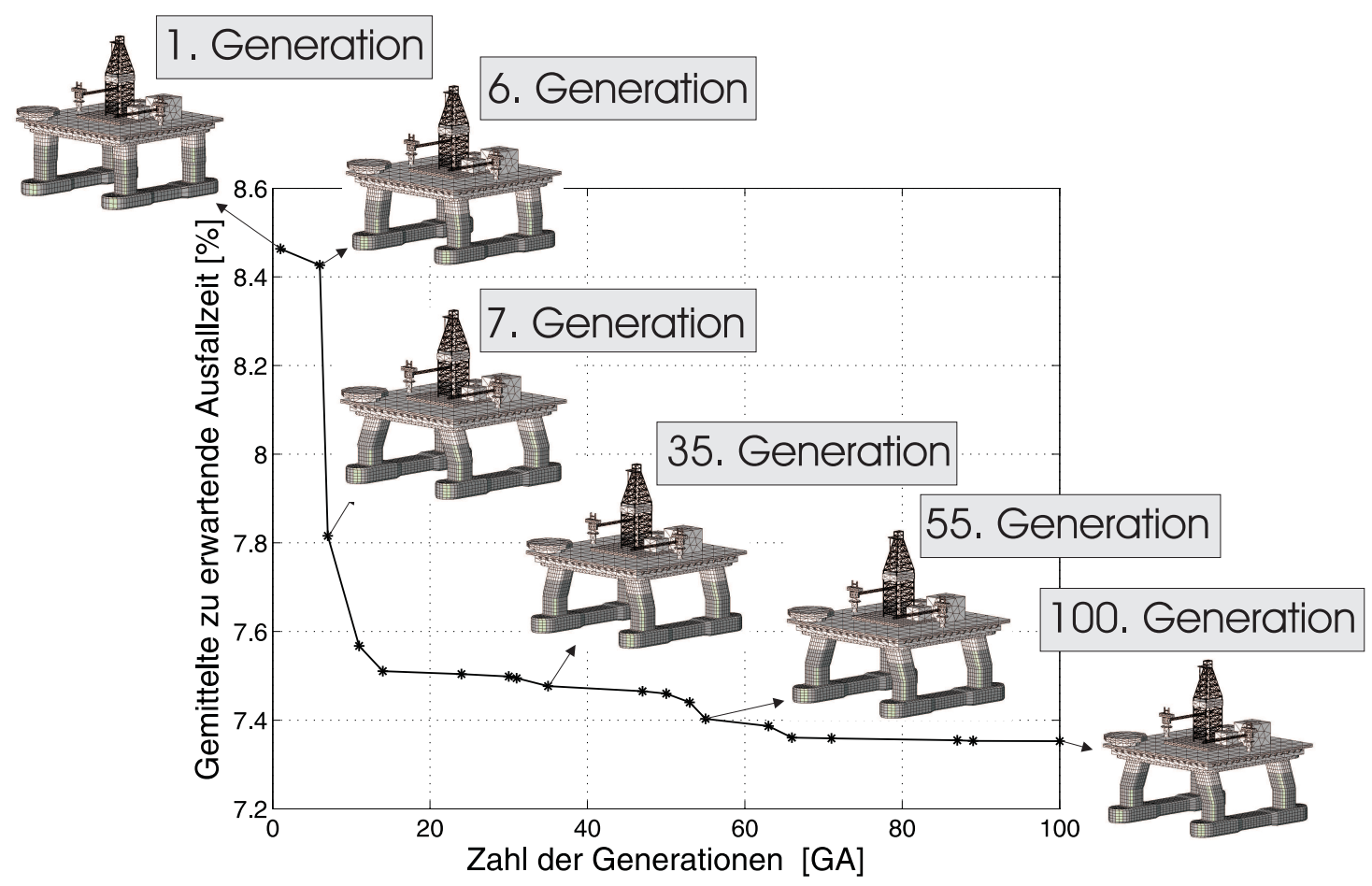

Abbildung 6.30: Entwicklung von Geometrie und Änderung der Zielfunktionswerte im Laufe der hydrodynamischen Formoptimierung mit einem Genetischen Algorithmus für den Halbtaucher mit $\triangle=50,000 \mathrm{~m}^{3}$

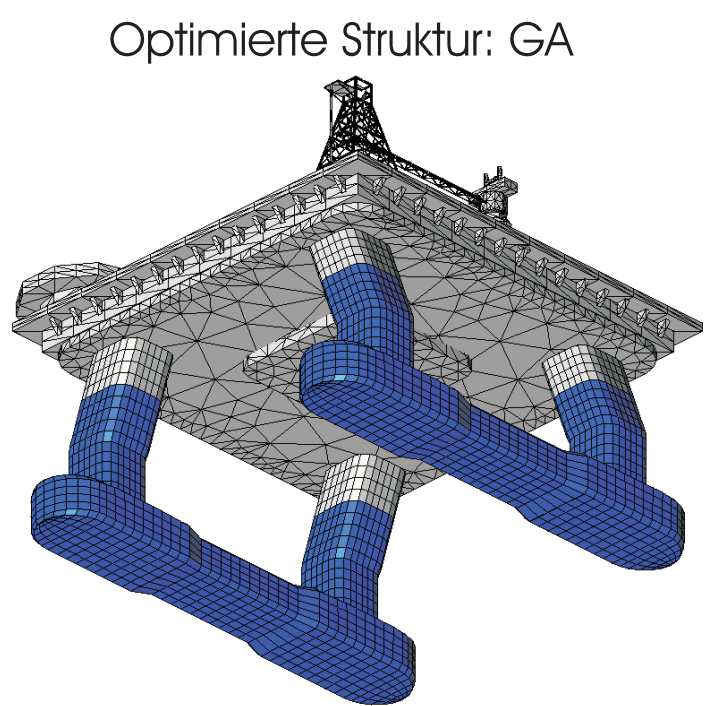

Gemittelte zu erwartende Ausfallzeit

$$
\overline{\mathrm{P}}_{d}=7.35 \%
$$

Optimierte Struktur: ASA

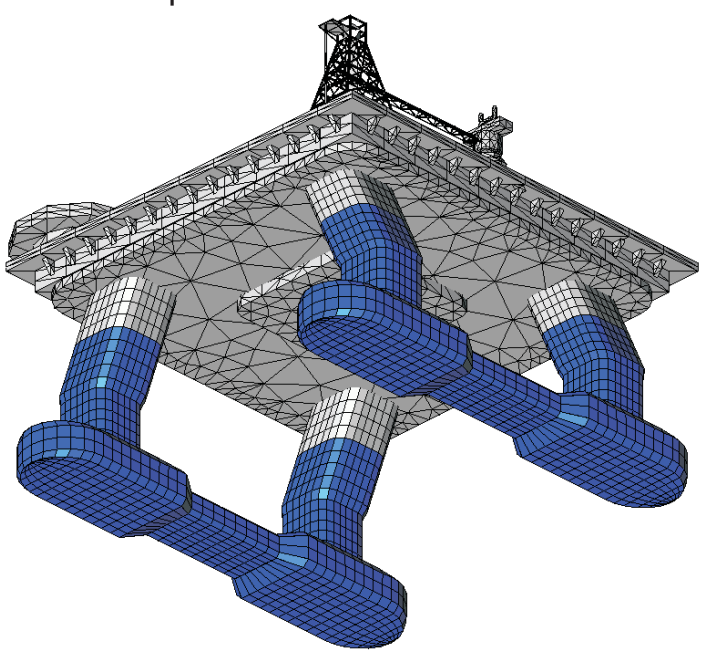

Gemittelte zu erwartende Ausfallzeit

$$
\overline{\mathrm{P}}_{d}=7.38 \%
$$

Abbildung 6.31: Ergebnis der globalen Optimierung eines hydrodynamisch kompakten Halbtauchers mit dem Genetischen Algorithmus (GA) und dem Adaptive Simulated Annealing Algorithmus (ASA) 


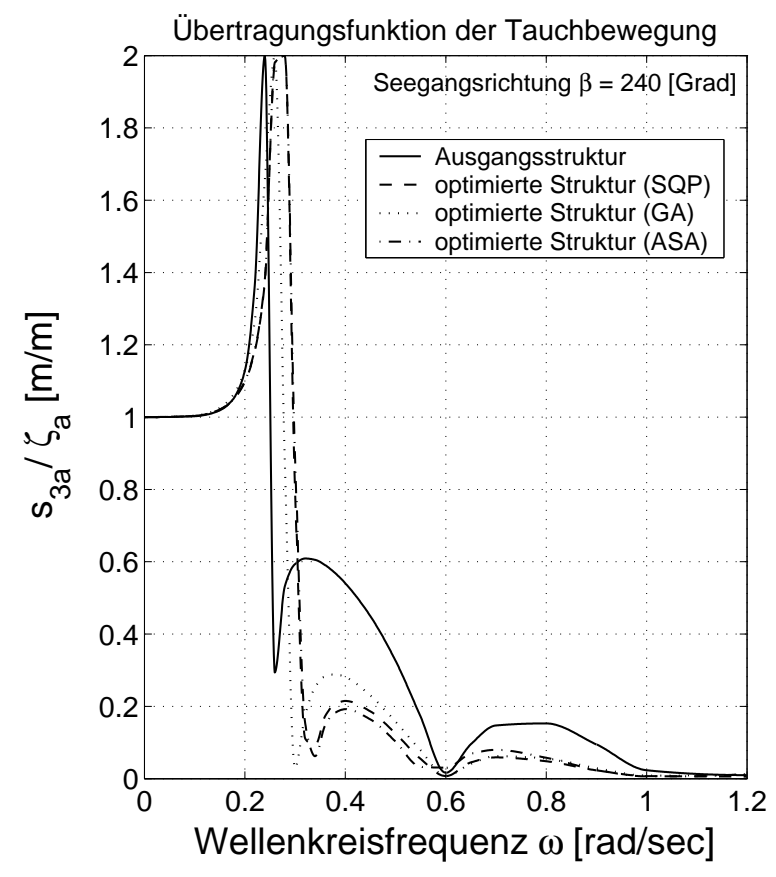

Abbildung 6.32: Übertragungsfunktionen der Tauchbewegung für die Ausgangsstruktur und die optimierten Strukturen bei Windrichtung $\beta=240$ [Grad]

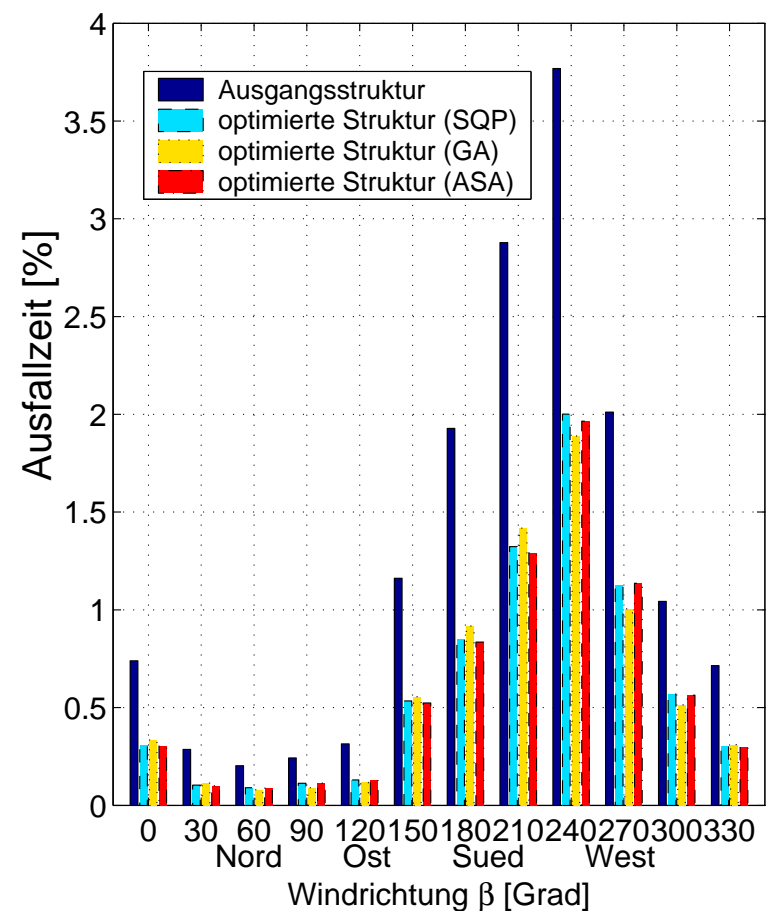

Abbildung 6.33: Verteilung der zu erwartenden Ausfallzeit nach Windrichtung $\beta$ im Nordostatlantik (Marsden Square 182) 
Minima als der mit SQP Algorithmus. Die Verbesserung der Zielfunktion für beide Minima ist jedoch mit $0.1 \%$ sehr gering. Der Genetische Algorithmus optimiert mit 1338 Funktionsauswertungen die gemittelte Ausfallzeit bis 7.35\%, wobei als Dauer der Evolution maximal 100 Generationen zugelassen wurde. Abb. 6.30 zeigt die Entwicklung der Geometrien anhand der jeweils besten Entwürfe ausgewählter Generationen und die Abnahme der Zielfunktionswerte im Laufe der hydrodynamischen Formoptimierung. Hierbei ist zu beobachten, dass während der Evolution die Veränderung der Geometrie mit abnehmender Wahrscheinlichkeit großer Mutationen geringer wird.

Der Adaptive Simulated Annealing Algorithmus erreicht vergleichsweise schneller ein Minimum etwa mit der Hälfte des Rechenaufwandes wie GA (siehe Tab.6.7). Die optimierte Struktur besitzt dabei fast den gleichen Zielfunktionswert mit $\bar{P}_{d}=7.38 \%$. Die Geometrien der beiden optimierten Strukturen von GA und ASA sind jedoch unterschiedlich (Abb.6.31, siehe auch Tab.6.8). Vor allem ist die Veränderung des Pontons bei der durch ASA optimierten Struktur deutlich. Mit beiden optimierten Strukturen konnte durch die nachgeschaltete Optimierung mit dem lokalen Algorithmus SQP keine weitere Verbesserung der Zielfunktion erzielt werden.

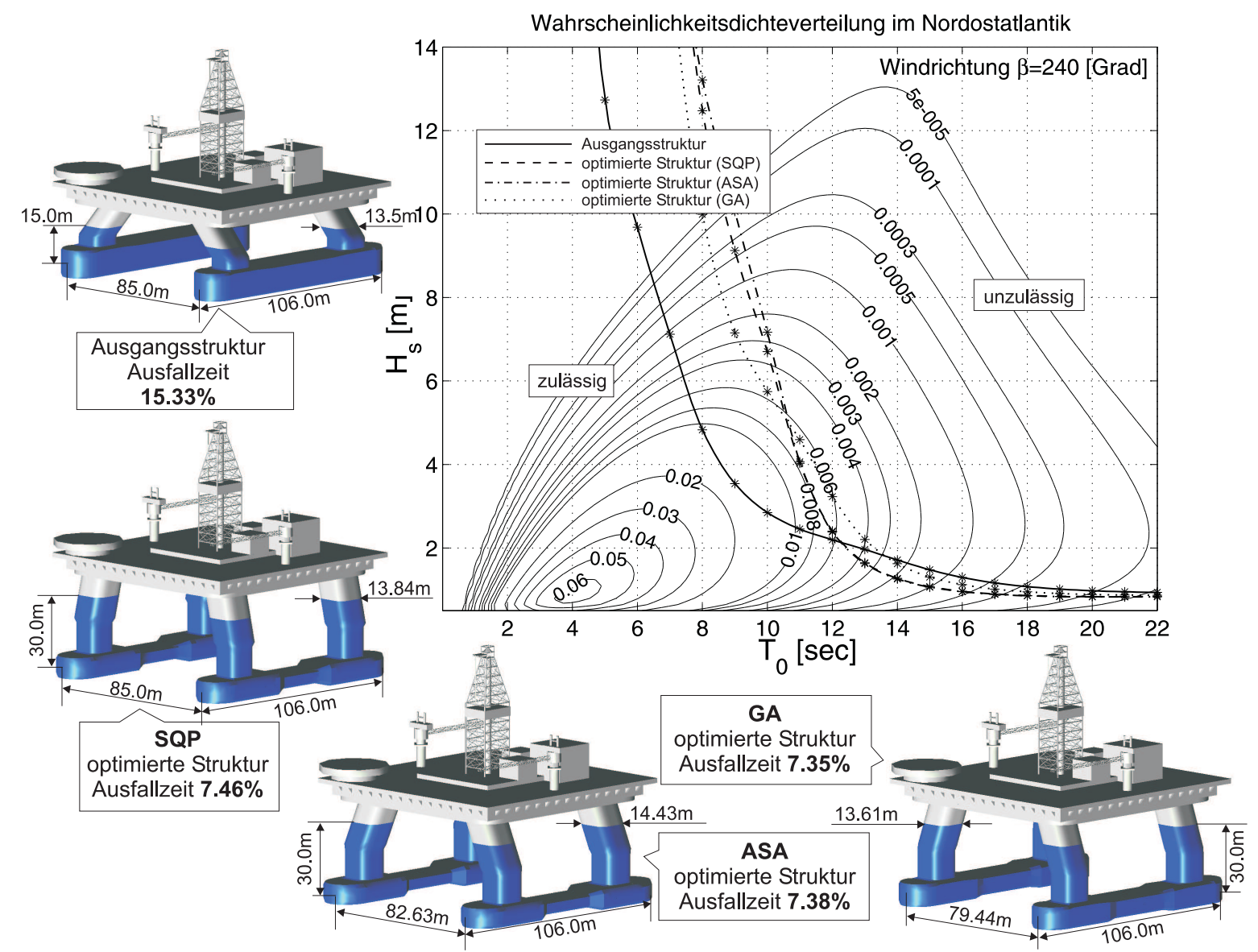

Abbildung 6.34: Vergleich der Geometrien und Grenzkurven der zulässigen signifikanten Wellenhöhen für die Ausgangsstruktur und die optimierten Strukturen bei Windrichtung $\beta=240[\mathrm{Grad}]$ 
Die durch die Formoptimierung gewonnenen Qualitätserhöhungen der hydrodynamischen Eigenschaften für die optimierten Strukturen zeigen sich deutlich in den Übertragungsfunktionen der Tauchbewegung für die häufigste Seegangsrichtung $\beta=240$ (Abb. 6.32). Bei der Resonnazstelle wird die Bewegung nur durch Potentialdämpfung gedämpft, da hierbei kein Zähigkeitseinfluss berücksichtigt wird. Für alle Seegangsrichtungen konnten auch die zu erwartenden Ausfallzeiten deutlich reduziert werden (Abb 6.33). Für mehrere Seegangsrichtungen z.B. $\beta=150^{\circ}, 180^{\circ}$ und $210^{\circ}$ weist das GA-Optimum im Vergleich zum ASA-Optimum größere zu erwartende Ausfallzeiten auf. Für die häufigste Seegangsrichtung $\beta=240^{\circ}$ ist jedoch die Einsatzzeit der durch GA optimierten Struktur größer als von ASA. Dadurch ergibt sich eine fast identische mittlere Ausfallzeit für GA und ASA.

Aufgrund der Unterschiede in den Übertragungsfunktionen der Tauchbewegung für die optimierten Strukturen erhält man auch unterschiedliche Grenzkurven der zulässigen signifikanten Wellenhöhen (Abb. 6.34). Obwohl für das GA-Optimum im Bereich der Zero-Upcrossing Perioden von $8 s<T_{0}<10.5 s$ die zulässigen signifikanten Wellenhöhen $H_{s, z u l}$ kleiner sind als die anderer optimierter Strukturen (SQP und ASA), wird im Bereich für $T_{0}>10.5 \mathrm{~s}$ die Grenzkurve von $H_{s, z u l}$ nach oben verschoben und damit der zulässige Bereich der Seegangszustände vergrößert. Die durch globale Algorithmen gefundenen Entwürfe ergeben somit zwei deutlich unterschiedliche Minima, die jedoch nur eine geringe Abweichung beim Zielfunktionswert haben.

\subsubsection{Tension Leg Plattform}

Das letzte Anwendungsbeispiel präsentiert die Optimierung eines zugspannungsverankerten Halbtauchers (Tension Leg Plattform -TLP), bei der als Zielfunktion der zu erwartende Ermüdungsschaden (fatigue damage) des Tethers für das Einsatzgebiet Haltenbanken minimiert werden soll. Mit einer Gesamtverdrängung $\triangle=105000 \mathrm{~m}^{3}$ besteht die Tension Leg Plattform aus vier Säulen, die durch schlanke Pontons verbunden sind (Abb. 6.35). Bei der Formoptimierung wird die Geometrie der TLP insgesamt mit 6 freien Variablen erzeugt:

- der Abstand zwischen Säulen $C_{\text {sep }}$,

- die Tauchtiefe der Pontonachse $D_{p}$,

- das Verhältnis der Querschnittsfläche von oberer und unterer Säule $A_{c o} / A_{c u}$,

- das Verhältnis der Länge von oberer und unterer Säule $L_{c o} / L_{c u}$,

- das Verhältnis des Säulenvolumens zur Gesamtverdrängung $\nabla_{c} / \nabla$,

- das Verhältnis von Breite zu Höhe des Pontons $B_{p} / H_{p}$.

Als unveränderliche Formparameter wird die Länge des Übergangs zwischen der oberen und der unteren Säule mit $L_{c i}=2.5 \mathrm{~m}$ definiert.

Zur Abschätzung der Ermüdungsschaden nach (5.29) und (5.30) müssen zunächst die Zugspannungen des Tethers berechnet werden. Bei früheren Arbeiten von Clauss 


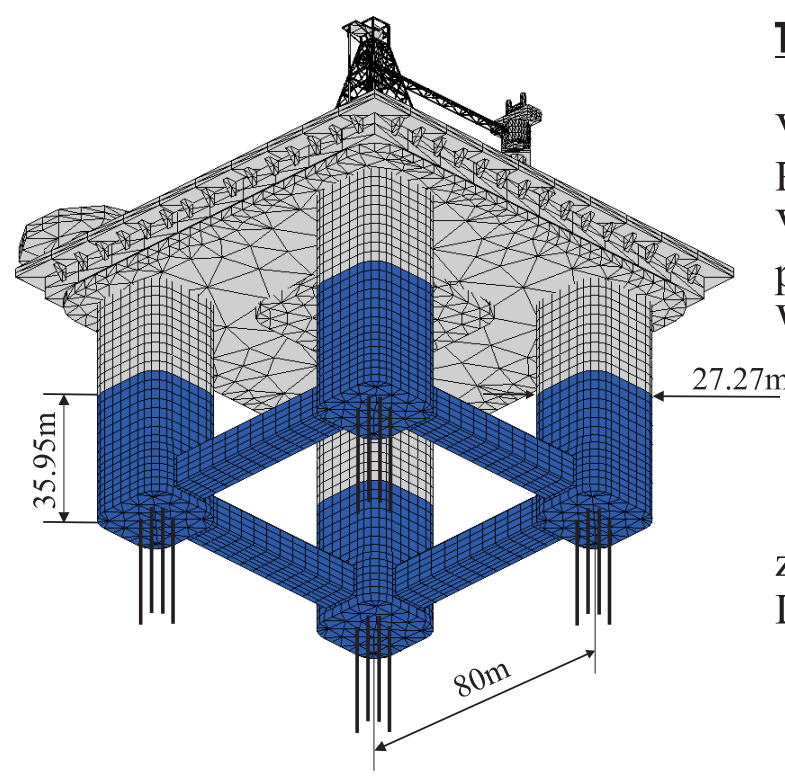

Tension Leg Plattform

Verdrängung $\Delta=105000 \mathrm{~m}^{3}$

Plattformgewicht $\mathrm{W}=78750 \mathrm{t}$

Vorspannung $\quad \mathrm{T}=64378 \mathrm{kN}$

pro eine Säule

Wassertiefe $\quad \mathrm{d}=310 \mathrm{~m}$

zu erwartende Schädigung $\mathrm{D}=0.0614$

Lebensdauer des Tethers 16 Jahre

\begin{tabular}{|c|c|}
\hline freie Variable & Ausgangsstruktur \\
\hline Abstand der Säulenachse $C_{s e p}$ & $80.0 \mathrm{~m}$ \\
Tauchtiefe der Pontonachse $D_{p}$ & $30.0 \mathrm{~m}$ \\
Verhältnis der Querschnittsflächen $A_{c o} / A_{c u}$ & 1.00 \\
Verhältnis der Länge $L_{c o} / L_{c u}$ & 1.00 \\
Verhältnis des Volumens $\triangle_{c} / \triangle$ & 0.80 \\
Verhältnis von Breite zu Höhe $B_{p} / H_{p}$ & 1.20 \\
\hline
\end{tabular}

Abbildung 6.35: Geometrie und Formparameter der Ausgangsstruktur für Tension Leg Plattform

und Birk (1996) und Birk (1998) wurden die Zugkräfte nur aus den Wellenkräften ermittelt und die Wechselwirkungen zwischen Tethern und Rumpf nicht berücksichtigt. Um die Resonanzerscheinungen des Systems einzubeziehen, wird ein mechanisches Modell betrachtet, wobei die TLP unter Welleneinwirkungen in ausgelenkter Lage dargestellt wurde (Abb. 6.36). Nach dem TLP-Konzept sollten Roll- bzw Stampfbewegungen aufgrund der großen Zugspannungen im Verankerungssystem kaum auftreten. Nur durch die unvermeidbare elastische Dehnung der Zuganker sind geringe Roll- und Stampfbewegungen möglich. In Abb.6.36 sind diese (nicht maßstabsgetreu) wiedergegeben, um sie sichtbar zu machen. Mit $T_{0}$ wird in Abb.6.36 die Vorspannung der Zuganker bezeichnet (pretension), die durch Auftriebsüberschuss entsteht:

$$
T_{0}=\frac{\triangle-W}{N_{C}}
$$

Dabei ist $\triangle$ die Verdrängung, $W$ das Gesamtgewicht der Plattform und $N_{C}$ die Anzahl der Säulen $\left(N_{C}=4\right)$. Die axiale Steifigkeit $k$ des Tethers definiert man mit dem Elastizitätsmodul $E$, der Querschnittsfläche des Tethers $A_{T}$ und der Länge des Tethers 


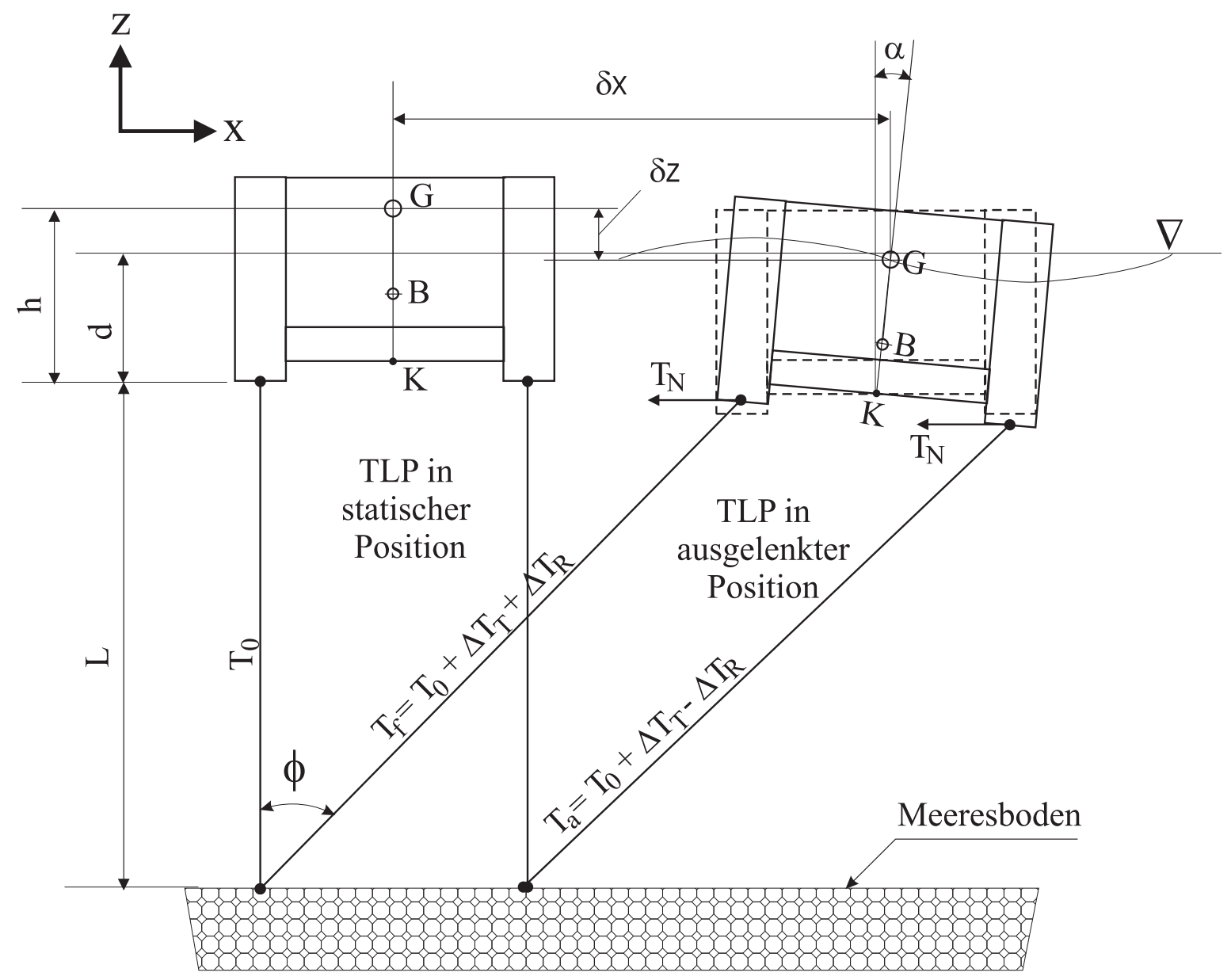

Abbildung 6.36: Mechanisches Modell für Tension Leg Plattform

$L:$

$$
k=N_{T} \frac{A_{T} E}{L}
$$

wobei $N_{T}$ die Zahl der Tether pro Säule ist.

Im Betrieb ändert sich die Zugkraft aufgrund translatorischer und rotatorischer Bewegungen der Plattform (Abb. 6.36). Ist $\Delta T_{T}$ die Änderung der Zugkraft aus translatorischer und $\Delta T_{R}$ die Änderung aus rotatorischer Bewegung, erhält man für die Zugkräfte der vorderen Tethergruppe $T_{f}$ und der hinteren Tethergruppe $T_{a}$ in ausgelenkter Lage:

$$
\begin{aligned}
T_{f} & =T_{0}+\Delta T_{T}+\Delta T_{R} \\
& =T_{0}+k\left(\varepsilon+\frac{C_{s e p}}{2} \alpha\right) \\
T_{a} & =T_{0}+\Delta T_{T}-\Delta T_{R} \\
& =T_{0}+k\left(\varepsilon-\frac{C_{s e p}}{2} \alpha\right) .
\end{aligned}
$$

Die Dehnung des Tethers $\varepsilon$ bezeichnet den Anteil infolge der Tauchbewegung und $C_{s e p} \alpha / 2$ den Anteil aus der Stampfbewegung. 
Bei der dynamischen Analyse mit WAMIT werden die Effekte des Tethers und die Auftriebskräfte des TLP-Körpers durch lineare Federn ersetzt. Im folgenden werden die äquivalenten Federkonstanten $C_{x}, C_{z}$ und $C_{\alpha}$ für die Tension-Leg Plattform zusammengefasst, die für die Definition der Matrix der Rückstellkoeffizienten $\underline{\underline{C}} \mathrm{im}$ Bewegungsgleichungssystem (B.104) verwendet werden:

- die vertikale Federkonstante $C_{z}$ :

Die axiale Steifigkeit besteht aus zwei Termen, d.h. aus der Steifigkeit des Tethers und aus der hydrostatischen Rückstellkraft:

$$
C_{z}=N_{C}\left(k+\rho g A_{c o}\right)
$$

wobei $\rho \widehat{=}$ Wasserdichte, $A_{c o} \widehat{=}$ die Querschnittsfläche der Säule

- die horizontale Federkonstante $C_{x}$ :

Aus der horizontalen Verschiebung $\delta_{x}$ erhält man die gesamte Rückstellkraft in horizontaler Richtung:

$$
N_{C} T_{N}=N_{C} T_{0} \sin \phi=N_{C} T_{0} \frac{\delta_{x}}{L} .
$$

Daraus ergibt sich das Rückstellkoeffizient in x-Richtung:

$$
C_{x}=\left(\frac{N_{C} T_{0}}{L}\right)
$$

- die rotatorische Federkonstante $C_{\alpha}$ :

$$
C_{\alpha}=\Delta \overline{K B}-W \overline{K G}+\frac{C_{z}}{N_{c}} C_{s e p}^{2}
$$

wobei $\overline{K B}$ : der Abstand vom Kiel $K$ zum Auftriebsschwerpunkt $B$

$\overline{K G}$ : der Abstand von $K$ zum Gewichtsschwerpunkt $G$

Während der Optimierung werden die Rückstellkoeffizienten bei jedem Optimierungsschritt neu berechnet, da sich die Federkennwerte nach der Variation der Geometrie ständig ändern. Bei der hydrodynamischen Analyse nach der Diffraktionstheorie wurden zur Begrenzung der Resonanzamplitude die konstanten Dämpfungen (Dämpfungs$\operatorname{grad} \delta \approx 1 \%$ ) für Tauch-, Roll- und Stampfbewegungen eingeführt. Setzt man die Lösungen der hydrodynamischen Analyse für $\varepsilon$ und $\alpha$ in (6.3) ein, erhält man die Zugkräfte $T_{f}$ und $T_{a}$ unter Welleneinwirkung. Mit der Einführung des Spannungskonzentrationsfaktor $S C F$ lässt sich nun die Übertragungsfunktion der dynamischen Spannungen für die vordere $\sigma_{f} / \zeta_{a}$ und für die hintere Tethergruppe $\sigma_{a} / \zeta_{a}$ folgendermaßen definieren:

$$
\begin{aligned}
& \frac{\sigma_{f}}{\zeta_{a}}=S C F \frac{k\left(\varepsilon+\frac{C s e p}{2} \alpha\right)}{\zeta_{a} A_{T} N_{T}} \\
& \frac{\sigma_{a}}{\zeta_{a}}=S C F \frac{k\left(\varepsilon-\frac{C s e p}{2} \alpha\right)}{\zeta_{a} A_{T} N_{T}}
\end{aligned}
$$


Die für die Ermüdungsanalyse notwendigen Daten sind in Tab. 6.9 dargestellt. Aus der Übertragungsfunktion von (6.9) folgt nach (5.11) das Antwortspektrum der dynamischen Spannungen für einen Seegang. Damit lässt sich schließlich der zu erwartende Gesamtschaden $D$ des Tethers nach (5.30) ermitteln. Für die genaue Abschätzung der Ermüdungsschädigungen der TLP reicht die lineare Analyse jedoch nicht aus, da bei Resonanzperioden für Stampf-, Roll- und Tauchbewegungen Ringing- und SpringingErscheinungen infolge extremer Wellenkräfte zweiter Ordnung auf die Lebensdauer des Tethersystems erheblichen Einfluss haben (Matten et al., 1996). Die absoluten Werte der Ermüdungsschäden sind deshalb mit Vorsicht zu bewerten. Dennoch bieten die Ergebnisse der linearen Spektralanalyse eine verlässliche Basis für den Vergleich unterschiedlicher Entwürfe.

\begin{tabular}{|c|c|}
\hline \multicolumn{2}{|c|}{ S-N Kurve } \\
\hline Ermüdungsgrenze (fatigue limit) & $\sigma_{D}=210 \mathrm{~N} / \mathrm{mm}^{2}$ \\
die Zahl der Lastzyklen bei $\sigma_{D}$ & $N_{D}=2.0 \cdot 10^{6}$ \\
der Exponent der Kurve & $k=3$ \\
\hline \multicolumn{2}{|c|}{ RAO der dynamischen Spannung } \\
\hline Spannungskonzentrationsfaktor & $S C F=10$ \\
die Zahl der Tether pro Säule & $N_{T}=4$ \\
Querschnittsfläche des Tethers & $A_{T}=92727 \mathrm{~mm}^{2}(\mathrm{D}=812.8 \mathrm{~mm}, \mathrm{t}=38.1 \mathrm{~mm})$ \\
\hline
\end{tabular}

Tabelle 6.9: Verwendete Daten für die Ermüdungsanalyse

\begin{tabular}{|c|c|c|c|}
\hline Algorithmen & $\begin{array}{l}\text { Anzahl der } \\
\text { Zielfunktionsbewertungen }\end{array}$ & $\begin{array}{l}\text { Gesamtschaden } \\
\text { D [1/Jahr }]\end{array}$ & $\begin{array}{l}\text { Lebensdauer } \\
\text { [Jahre }]\end{array}$ \\
\hline \multicolumn{2}{|c|}{ Ausgangsstruktur } & 0.0614 & 16 \\
\hline SQP & 71 & 0.0155 & 65 \\
GA & 401 & 0.0152 & 66 \\
ASA & 518 & 0.0120 & 83 \\
GA+SQP & $401+139$ & 0.0118 & 85 \\
ASA+SQP & $518+47$ & 0.0120 & 83 \\
\hline
\end{tabular}

Tabelle 6.10: Vergleich der Ergebnisse der hydrodynamischen Formoptimierungen für eine Tension Leg Plattform

Wie die Ergebnisse der Optimierungen in Tab. 6.10 zeigen, konnte der Ermüdungsschaden durch die Formoptimierung deutlich verringert werden. Der lokale SQPAlgorithmus minimiert bereits nach 71 Schritten den Gesamtschaden auf 25\%, d.h. erhöht die Lebensdauer des Tethers von 16 Jahren auf 65 Jahre. Durch den Einsatz globaler Algorithmen konnte die zu erwartende Lebensdauer auf 85 Jahre maximiert werden, wobei die benötigte Anzahl der Funktionsbewertungen wesentlich höher ist als bei SQP. Bei Anwendung des Genetischen Algorithmus konvergiert die Lösung langsam, besonders wenn das Minimum mit hoher Genauigkeit lokalisiert werden soll. Deshalb 
wird hier zunächst nur insgesamt über 30 Generationen die Optimierung durchgeführt, um ein potenzielles Minimum zu finden, das sich als bestes Individuum auszeichnet. Anschließend findet die deterministische Suchstrategie SQP mit diesem Anfangsvektor das beste Minimum. Ausgehend vom Minimum nach dem ASA Verfahren wurde ebenfalls die nachgeschaltete Optimierung mit Hilfe des SQP Verfahren durchgeführt, wobei eine kaum erkennbare Verbesserung der Zielfunktion erreicht werden konnte.

Abb. 6.37 vergleicht die Geometrien und geschätzte Lebensdauern der Zuganker der optimierten Tension Leg Plattformen. Die Abstände zwischen den Säulen und die Tauchtiefe der Pontonachse wurden teilweise bis zur vordefinierten Grenze $C_{\text {sep }}=85 \mathrm{~m}$ und $D_{p}=40 \mathrm{~m}$ vergrößert. Auch die Länge und die Querschnittsfläche des oberen Teils der Säulen wurden leicht vergrößert. Die Unterschiede der mit globalen Optimierungsalgorithmen maximierten Lebensdauer sind gering. Dies spiegelt sich auch in geringen Unterschieden bei den Formparametern wider.

Zur Bewertung der durch die Formoptimierung erzielten Verringerung der Ermüdungsschäden werden die Übertragungsfunktionen der dynamischen Spannungen der Zuganker sowie die Verteilungen der Schädigungen im Seegang für die Ausgangsstruktur und die optimierten Strukturen verglichen. In Abb. 6.38 zeigt sich, dass die dynamischen Spannungen des Tethers besonders deutlich im Frequenzbereich von $0.4<\omega<0.6$ reduziert werden. Bei der Ausgangsstruktur liegt die Resonanzfrequenz für die Dehnung des Tethers infolge der Tauchbewegung etwa bei $\omega=0.58 \mathrm{rad} / \mathrm{s}$. Deshalb tritt hier die maximale dynamische Spannung des Tethers auf. Für alle optimierten Strukturen werden die Resonanzfrequenzen mit $\omega=0.6 \mathrm{rad} / \mathrm{s}$ leicht verschoben. Die Resonanzamplituden der dynamischen Spannungen werden dabei wesentlich verkleinert, weil die Wellenkräfte durch optimale Änderungen an der Geometrie reduziert wurden. Im niedrigen Frequenzbereich $\omega<0.3 \mathrm{rad} / \mathrm{s}$ hat die Formoptimierung erhöhte Tetherspannungen zur Folge. Dies trägt aber nicht wesentlich zur Erhöhung der Schädigungen bei, da im betrachteten Seegebiet die Häufigkeit langer Wellen gering ist. Wie auch die Verteilungen der Schädigungen in Abb. 6.39 eindrucksvoll zeigen, konzentrieren sich die Schädigungen der Zuganker auf den Bereich von $T_{0} \in[7 s, 8 s]$. Aus der Zero-up-crossing Periode von $T_{0}=7.5$ ergibt sich die so genannte Modalperiode zu $T_{p}=1.408 \cdot T_{0}=10.56 \mathrm{~s}$ bzw. die Peakfrequenz zu $\omega \approx 0.6$ (Faltinsen, 1990). Die Optimierung verringert die Spannungen in diesem kritischen Bereich um gut $40 \%$. 


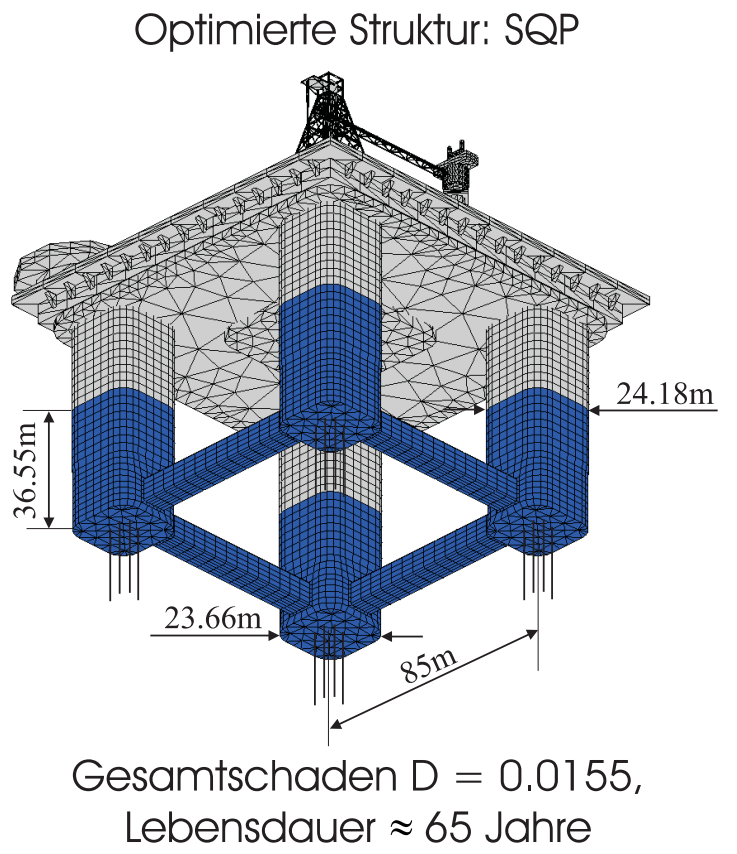

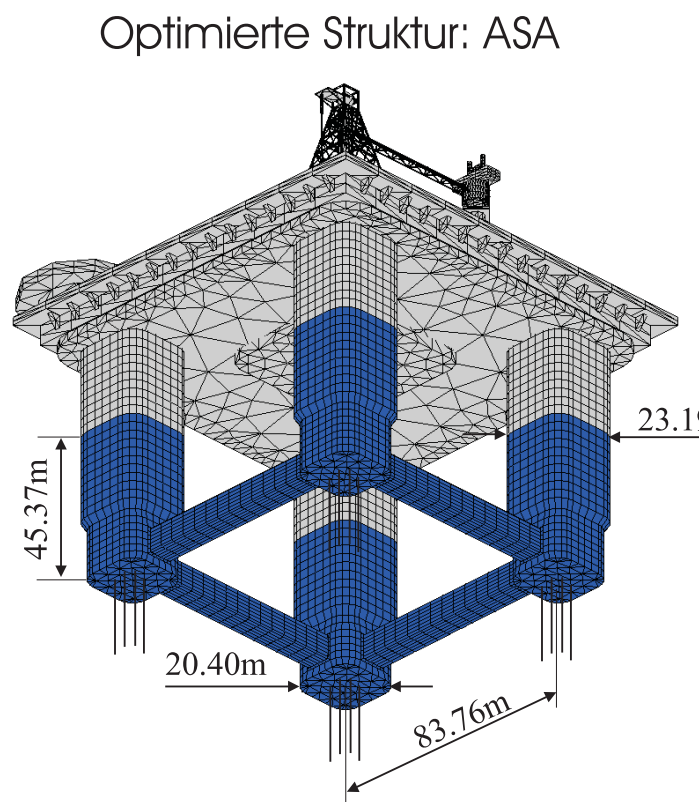

Gesamtschaden D $=0.0120$, Lebensdauer $\approx 83$ Jahre
Optimierte Struktur: GA+SQP

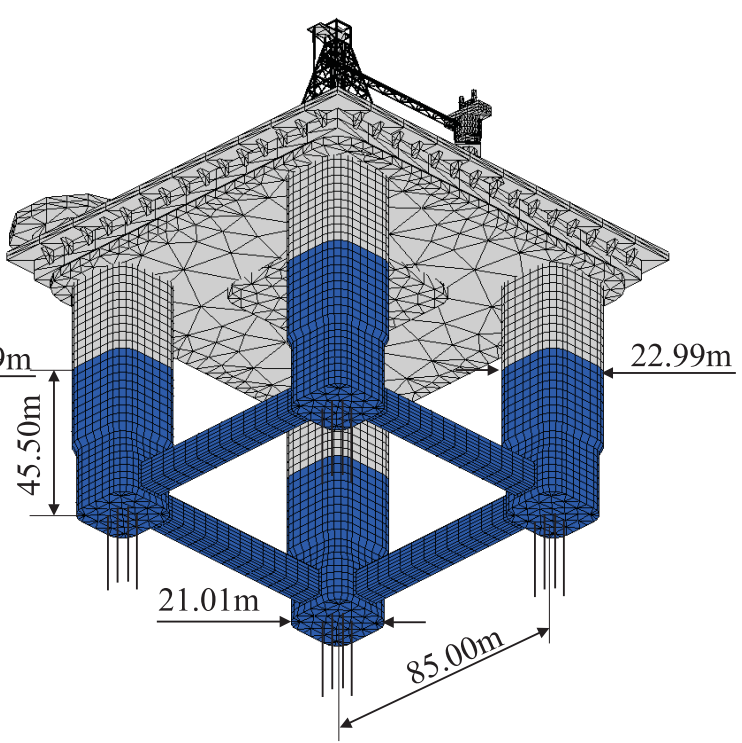

Gesamtschaden D $=0.0118$, Lebensdauer $\approx 85$ Jahre

\begin{tabular}{|l|cccccc|}
\hline \multirow{3}{*}{ Algorithmen } & \multicolumn{6}{|c|}{ freie Variable } \\
\cline { 2 - 7 } & $C_{\text {sep }}$ & $D_{p}$ & $A_{c o} / A_{c u}$ & $L_{c o} / L_{c u}$ & $\nabla_{c} / \nabla$ & $B_{p} / H_{p}$ \\
& {$[m]$} & {$[m]$} & {$[\cdot]$} & {$[\cdot]$} & {$[\cdot]$} & {$[\cdot]$} \\
\hline SQP & 85.00 & 33.71 & 1.05 & 1.01 & 0.8500 & 1.10 \\
ASA & 83.76 & 39.98 & 1.29 & 1.71 & 0.8492 & 1.04 \\
GA+SQP & 85.00 & 40.00 & 1.20 & 1.20 & 0.8460 & 1.00 \\
\hline
\end{tabular}

Abbildung 6.37: Vergleich der Geometrien und Lebensdauern für die optimierten Strukturen: TLP 


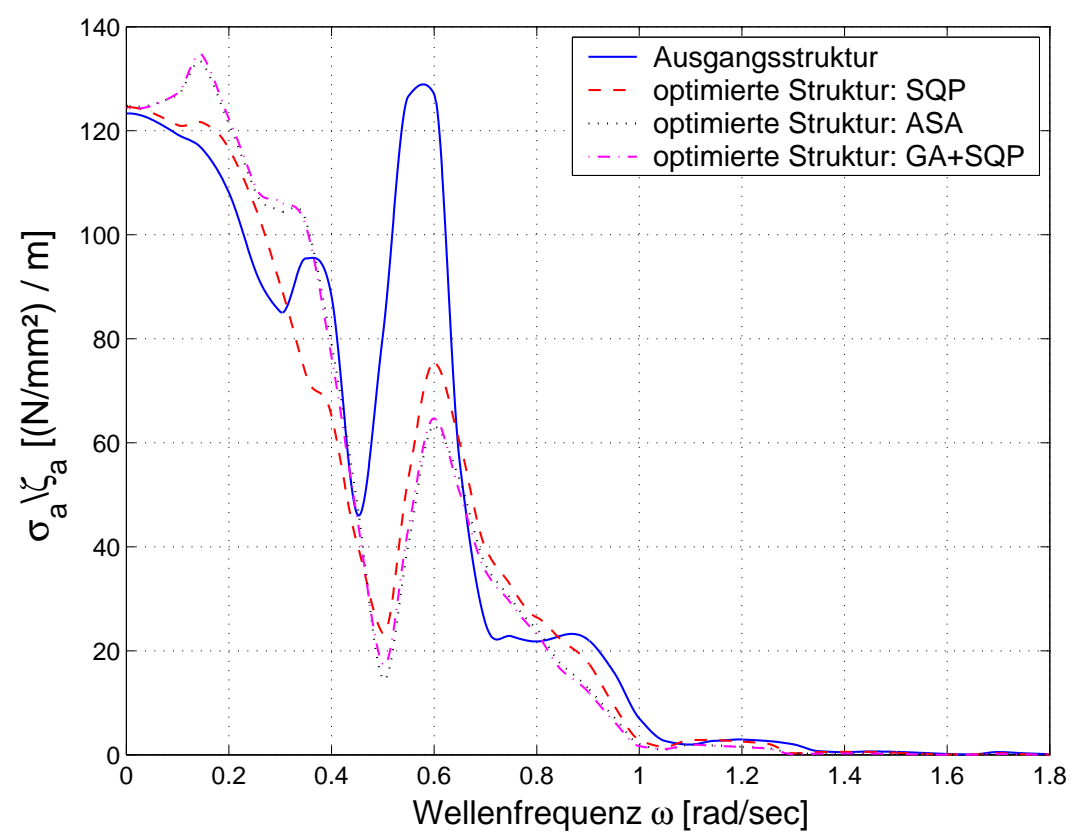

Abbildung 6.38: Vergleich der Übertragungsfunktionen der dynamischen Spannungen der Tether
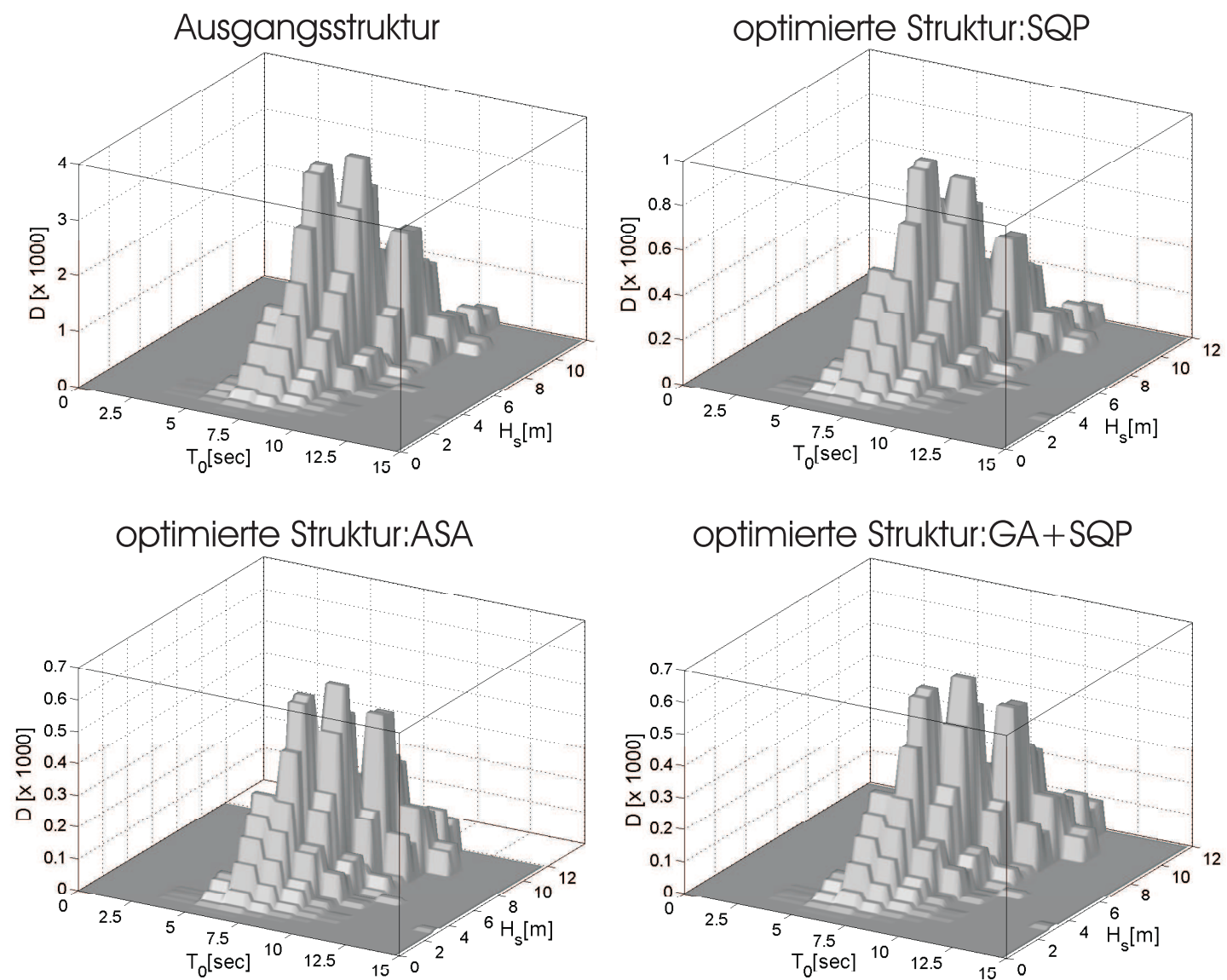

Abbildung 6.39: Vergleich der Verteilungen der Schädigungen für das HaltenbankenGebiet 


\section{Kapitel 7}

\section{Schluss}

Das System der hydrodynamischen Formoptimierung meerestechnischer Konstruktionen beruht auf drei eigenständigen numerischen Werkzeugen, der Geometriemodellierung (CAD), der hydrodynamischen Analyse (CFD) und der Wahrscheinlichkeitstheorie zur Abschätzung der hydrodynamischen Eigenschaften im unregelmäßigen Seegang. Das Gesamtsystem wird durch Optimierungsalgorithmen gesteuert, um die Seegangseigenschaften in einem automatisierten Prozess zu verbessern. Ausgehend von einem vorgegebenen Entwurf oder zufällig ausgewählter Ausgangsstrukturen werden Formvariationen durchgeführt, bis ein Minimum der Zielfunktion gefunden ist. In der vorliegenden Dissertationsschrift wurden unterschiedliche Klassen von globalen Optimierungsstrategien in das hydrodynamische Formoptimierungssystem integriert, um sie hinsichtlich Aufwand und Leistungsfähigkeit zu vergleichen.

Zur Vergrößerung der Formenvielfalt wird bei der hydrodynamischen Formoptimierung ein erweitertes Formgenerierungsverfahren eingesetzt (Huang, 2000). Die Beschreibung der Topologie und der geometrischen Parameter erfolgt mittels einer eigenen Definitionssprache, die auf Python (van Rossum und Drake Jr., 2003) basiert. Dadurch lassen sich Zusammenhänge zwischen einzelnen Formparametern einfach und flexibel darstellen. Aus den Formparametern werden die Grundkurven einer Strukturkomponente definiert und damit die Oberfläche der Komponente durch eine modifizierte Sweeping-Operation in Form einer Non-Uniform-Rational-B-Spline (NURBS) Fläche automatisch erzeugt, ohne jeglichen Eingriff des Benutzers.

Bei der hydrodynamischen Formoptimierung werden sowohl hydrodynamisch transparente als auch hydrodynamisch kompakte Konstruktionen als Anwendungsbeispiele betrachtet. Für die Analyse hydrodynamisch transparenter Strukturen wird das auf der Basis der vektoriellen Morisongleichung entwickelte TFC (Transfer Function Calculation) Programm eingesetzt, mit dem die Übertragungsfunktionen für Kräfte und Bewegungen weitgehend analytisch und dadurch sehr viel schneller berechnet werden können als mit der numerisch aufwendigen hydrodynamischen Analyse nach einem 3D-Panelverfahren. Da das TFC-Programm die linearisierte zähigkeitsbedingte Widerstandskraft einbezieht, ist es möglich, den Einfluss der Zähigkeit auf die Optimierung zu berücksichtigen. Im Gegensatz zu den transparenten Strukturen können bei hydrodynamisch kompakten Strukturen die durch Reflexion und Diffraktion bedingten Seegangskräfte nicht mehr vernachlässigt werden. Für die Auswertung der Zielfunk- 
tion wird hierfür das 3D-Diffraktion-Radiations-Programm WAMIT verwendet, mit dem die zuverlässige Analyse der Kräfte und Bewegungen beliebig geformter Körper im Seegang möglich ist.

Durch die Analyse werden zur hydrodynamischen Bewertung die relativen Häufigkeiten der Seegangszustände auf Grundlage der Langzeit-Seegangsstatistik ermittelt, bei denen der operationelle Betrieb unterbrochen werden muss. Die Summation dieser Seegangszustände, d.h. die seegangsbedingte Ausfallzeit, wird als Gütekriterium für die Formoptimierung herangezogen. Als Zielfunktion wird auch die mittlere Ausfallzeit gewählt, die für die nach Windrichtungen aufgeteilten Seegangsstatistiken ermittelt wird. Hierbei wird die diskrete Tabelle für eine statistische Verteilung der Seegangszustände mit Hilfe einer numerischen Interpolation durch eine analytische Verteilungsfunktion ersetzt. Dadurch kann die mittlere Einsatzzeit einer Struktur in einem vorgegebenen Seegebiet bestimmt werden, ohne dass der Grenzwert für die zulässige signifikante Wellenhöhe auf die Klasseneinteilung der Tabelle gerundet werden muss. Außerdem werden Ermüdungsschäden als Zielfunktion in die hydrodynamische Formoptimierung einbezogen, um die Lebensdauer ausgewählter Konstruktionselemente zu maximieren. Alle Zielfunktionen werden auf Basis einer linearen, spektralen Analyse im Frequenzbereich durchgeführt.

Wie bereits für lokale Algorithmen gezeigt (Birk, 1998), können auch globale Strategien für die hydrodynamische Formoptimierung meerestechnischer Konstruktionen herangezogen werden. Der Rechenaufwand (die Anzahl der Zielfunktionsbewertungen) ist bei globalen Strategien wie erwartet deutlich höher. Wie die in Kapitel 6 illustrierten Anwendungsbeispiele zeigen, findet der lokale Algorithmus immer nur lokale Minima, welche von der ausgewählten Ausgangsstruktur abhängen. Im Gegensatz dazu konnten globale Optimierungsstrategien bzw. die Kombination von globalen und lokalen Algorithmen fast in allen Fällen bessere Minima lokalisieren. Der Beweis dafür, ob globale Optimierungsalgorithmen tatsächlich das gesuchte globale Optimum gefunden haben, kann nur durch eine Problemanalyse möglich sein, wobei hinreichende Informationen über multimodale Zielfunktionen zur Verfügung gestellt werden müssen. In der Umgebung der jeweils von verschiedenen Optimierungsalgorithmen gefundenen Minima müssen zumindest die Konvexität der Zielfunktion und der Nebenbedingungen untersucht werden.

Lokale Algorithmen sind besonders effektiv, wenn nur kleine Veränderungen des Ausgangsentwurfs zu erwarten sind, z.B. wenn ein bereits optimierter Entwurf an eine nur wenig veränderte Aufgabenstellung angepasst werden soll. Der Mehraufwand beim Einsatz globaler Optimierungsstrategien wird sich besonders dann lohnen, wenn neuartige Aufgabenstellungen zu lösen und neue Entwürfe anzufertigen sind, bei denen der Erfahrungsschatz noch gering ist. Von den getesteten globalen Algorithmen hat sich das Adaptive Simulated Annealing (Ingber, 1996) besonders gut bewährt. Es hat bei allen Testproblemen und bei den meisten Anwendungen bessere Ergebnisse erzielt, während der Einsatz des Genetischen Algorithmus in manchen Fällen nur ein lokales Minimum erbrachte (siehe auch Birk et al.,2002).

Offensichtlich beeinflussen die Zähigkeitsdämpfung und -kräfte den Ablauf und das Optimierungsergebnis, da die Veränderung der Übertragungsfunktion im Bereich der 
Resonanz- und Auslöschungsfrequenzen eine Auswirkung auf die Zielfunktion hat. Wie das Optimierungsbeispiel eines Halbtauchers mit einer Verdrängung von 30,000 $\mathrm{m}^{3}$ zeigt, sind die Unterschiede in den Geometrien der optimierten Strukturen deutlich (siehe Abschnitt 6.1.4). Vor allem spielt der Einfluss der Zähigkeit auf die Antwort der Strukturbewegungen im extremen Seegang mit $T_{0}>10$ eine große Rolle. Im Bereich der Frequenz $\omega<0.5$ liegen die Resonanz- und Auslöschungsfrequenz von Halbtauchern, wobei das Seegangsspektrum in diesem Bereich die meiste Energie besitzt.

Ein wichtiger Schritt bei der weiteren Integration von numerischen Werkzeugen in den Entwurfsprozess wird die Kopplung von hydrodynamischer und festigkeitsorientierter Optimierung sein. Die grundlegende Frage der parametrischen Modellierung der inneren, tragenden Struktur stellt hierbei die größte Hürde dar, die es in den kommenden Jahren zu überwinden gilt. Neben den technischen Zielfunktionen wird auch die Einbeziehung ökonomischer Zielfunktionen, z.B. Ausfallkosten ein wichtiger Schritt in der Optimierung von Offshore-Strukturen sein. 


\section{Literaturverzeichnis}

Abt, C., Bade, S., Birk, L., und Harries, S. (2001). Parametric hull form modelling a step towards one week ship design. In Proc. of $8^{\text {th }}$ Int. Symposium on Practical Design of Ships and other Floating Structures (PRADS 2001), Shanghai, China.

Akagi, S. und Ito, K. (1984). Optimal design of semisubmersible form by minimizing its motion in random seas. Journal of Mechanisms, Transmissions, and Automation in Design, Transactions of the ASME, 106:23-30.

Arciszewski, T., De Jong, K., und Vyas, H. (1999). Inventive design in structural engineering: evolutionary computation approach. In $5^{\text {th }}$ Int. Conf. on the applications of AI to Civil and Structural Engineering, S. 1-9. Oxford, UK.

Barltrop, N. und Adams, A. (1991). Dynamics of Fixed Marine Structures. Butterworth-Heinemann Ltd, Oxford, $3^{\text {rd }}$ edition.

Bennage, W. und Dhingra, A. (1995). Single and multiobjective structural optimization in descrete-continous variables using simulated annealing. Int. Jour. for Numerical Methods in Engineering, 38:2753-2773.

Bentley, P. und Wakefield, J. (1996). Overview of generic evolutionary design systems. In Proceedings of $2^{\text {nd }}$ On-Line World Conference on Evolutionary Computation (WEC2), S. 53-56.

Bertsekas, D. (1999). Nonlinear Programming. Athena Scientific, Belmont, MA, $2^{\text {nd }}$ edition.

Birk, L. (1990a). Die Berechnung der Bewegungsübertragungsfunktion für hydrodynamisch transparente Konstruktionen. Studienarbeit im Fach Meerestechnik, Institut für Schiffs- und Meerestechnik, TU Berlin.

Birk, L. (1990b). Vergleich von numerischer und experimenteller Analyse der Hydrodynamik eines Halbtauchers. Master's thesis, Technische Universität Berlin.

Birk, L. (1991). Vergleich von numerischer und experimenteller Analyse der Hydromechanik von Halbtauchern. Jahrbuch der Schiffbautechnischen Gesellschaft (STG), Bd. 85 .

Birk, L. (1998). Hydrodynamic Shape Optimization of Offshore Structures. Dissertation, Technische Universität Berlin.

Birk, L. (2003). Introduction to nonlinear programming. In OPTIMISTIC - Optimization in Marine Design, 39 ${ }^{\text {th }}$ WEGEMT Summer School, Kapitel 3, S. 53-82. Mensch \& Buch Verlag, Berlin. 
Birk, L. und Clauss, G. (1999). Efficient development of innovative offshore structures. In Offshore Technology Conference, Houston, Texas. OTC 10774.

Birk, L. und Clauss, G. (2001a). Automated hull optimisation of offshore structures based on rational seakeeping criteria. In Proc. of $11^{\text {th }}$ Int. Offshore and Polar Engineering Conference (ISOPE-2001), Stavanger, Norway.

Birk, L. und Clauss, G. (2001b). Rational design criteria and their application to hull form optimisation of floating systems in random seas. In Proc. of $8^{\text {th }}$ Int. Symposium on Practical Design of Ships and Other Floating Structures (PRADS 2001), Shanghai, China.

Birk, L., Clauss, G., und Lee, J. Y. (2002). Strategien zur hydrodynamischen Formoptimierung meerestechnischer Konstruktionen. In Jahrbuch der Schiffbautechnischen Gesellschaft, Hamburg, Germany.

Birk, L. und Harries, S. (2000). Automated optimization - a complementing technique for the hydrodynamic design of ships and offshore structures. In $1^{\text {st }}$ Conf. on Computer Applications and Information Technology in the Maritime Industries (COMPIT), Potsdam, Germany.

Boggs, P. und Tolle, J. (1995). Sequential Quadratic Programming. In Acta Numerica, S. 1-52. Springer Verlag.

Brebbia, C. und Walker, S. (1979). Dynamic Analysis of Offshore Structures. NewnesButterworths, London Boston.

Breit, S., Newman, J., und Sclavounos, P. (1985). A new generation of panel programs for radiation-diffraction problems. In Battjes, J., editor, Behaviour of Offshore Structures BOSS'85, Delft, The Netherlands.

Cartwright, D. und Longuet-Higgins, M. (1956). The statistical distribution of the maxima of a random function. In Proc. Royal Society, S. p.212-232. A237 (1209).

Catley, D., Lehmann, E., Maniar, N., Baret, B., Homma, Y., und Trafalski, W. (1990). Design optimization: A state of the art review. Marine Structures, 3(5):343-390.

Cerny, V. (1985). A thermodynamical approach to the travelling salesman problem: An efficient simulation algorithm. Journal of Optimization Theory and Application, $45: 41-51$.

Chakrabarti, S., Tam, W., und Wolpert, A. (1975). Wave forces on a randomly oriented tube. In Offshore Technology Conference, Volume 1, Houston.

Chou, F. (1977). A minimization scheme for the motions and forces of an ocean platform in random seas. SNAME Transactions, 85:32-50.

Clauss, G., Lehmann, E., und Östergaard, C. (1992). Offshore Structures, Volume 1: Conceptual Design and Hydrodynamics. Springer Verlag London.

Clauss, G. und Birk, L. (1994a). Hydrodynamic optimization of large offshore structures. In Proc. of $5^{\text {th }}$ Int. Marine Design Conference (IMDC '94), S. 595-608, Delft, The Netherlands.

Clauss, G. und Birk, L. (1994b). Optimizing the motion behaviour of offshore struc- 
tures. In Proc. of th Int. Conf. on Behaviour of Offshore Structures (BOSS '94), Volume 2 - Hydrodynamics and Cable Dynamics, S. 665-684, Cambridge (MA), USA.

Clauss, G. und Birk, L. (1995a). Design optimization of large offshore structures by nonlinear programming. In IX Int. Symp. on Offshore Eng. , BRASIL OFFSHORE '95, Rio de Janeiro, Brasil.

Clauss, G. und Birk, L. (1995b). Hydrodynamische Formoptimierung von Ein- und Mehrkörpersystemen im Seegang. Techn. Bericht TUB/ISM 95/6, Institut für Schiffs- und Meerestechnik, TU Berlin. DFG Cl 35/11-2.

Clauss, G. und Birk, L. (1996). Hydrodynamic shape optimization of large offshore structures. In Applied Ocean Research,18(4):15\%-171.

Clauss, G. und Birk, L. (1998a). Design of optimum offshore structures based on long term wave statistics. In Proc. of $17^{\text {th }}$ Int. Conf. on Offshore Mechanics and Arctic Engineering (OMAE '98), Lisbon, Portugal. ASME.

Clauss, G. und Birk, L. (1998b). Downtime minimization by optimum design of offshore structures. In Proc. of th $^{\text {th }}$ Practical Design of Ships and Mobile Units, Volume 11 von Development in Marine Technology, S. 1091-1070, The Hague, The Netherlands. Elsevier Science B.V.

Clauss, G., Birk, L., und Lee, J. Y. (2002). Hydrodynamische Formoptimierung meerestechnischer Konstruktionen unter Einsatz globaler Optimierungsstrategien. Zwischenbericht DFG Cl 35/28, Institut für Land- und Seeverkehr, Technische Universität Berlin.

Clauss, G. und Kühnlein, W. (1994). Seekeeping tests of marine structures with deterministic wave groups and tank side wall wave absorbers. In Proceedings of BOSS Conference, Cambridge, Massachusetts. Massachusetts Institute of Technology.

Cooper, L. und Glass, H. (1965). Sequential Search: A method for solving constrained optimization problems. Journal of Applied Computational Mathematics, 12:71-82.

Crandall, S. H. und Mark, W. D. (1973). Random Vibration in Mechanical Systems. Academic Press.

Cvetkovic, D. und Parmee, I. (1999). Genetic algorithms based systems for conceptual engineering design. In $12^{\text {th }}$ International Conference on Engineering Design (ICED'99), Volume 2, S. 1035-1038, München, Germany.

Deb, K. (1999). Evolutionary algorithms for multicriterion optimization in engineering design. In Evolutionary Algorithms in Engineering and Computer Science. Wiley \& Sons. pp 135-161.

Epperly, T. (1995). Global optimization of nonconvex nonlinear programs using parallel Branch and Bound. Dissertation, University of Wisconsin - Madison.

Evans, J. (1959). Basic design concepts. Journal of the American Society of Naval Engineers, 71(4):671-678.

Faltinsen, O. (1990). Sea loads on ships and offshore structures. Ocean Technology. 
Cambridge University Press.

Fiacco, A. und McCormick, G. (1968). Nonlinear Programming: Sequential Unconstrained Minimization Techniques. John Wiley and Sons, New York.

Fletcher, R. (1987). Practical Methods of Optimization. John Wiley \& Sons, New York, $2^{\text {nd }}$ edition.

Floudas, C., Androulakis, I., und Visweswaran, V. (1996). Distributed decompositionbased approaches in global optimization. In Floudas, C. und Pardalos, P., editors, State of the Art in Global Optimization: Comp. Methods and Applications, Book Series on Nonconvex Optimization and its Application. Kluwer Academic Publishers, Boston.

Fricke, W., Patzold, H., und Petershagen, H. (1997). Fatigue strength of ship structures - Part I: basic principles. Techn. Bericht 1/97, Germanischer Lloyd Group, Hamburg, Germany.

Gill, P., Murray, W., Saunders, M., und Wright, M. (1982). User's guide for sol/qpsol: a FORTRAN package for quadratic programming. Techn. Bericht SOL 82-70, Dept. of Operations Research, Stanford University, Stanford, CA.

Gill, P., Murray, W., Saunders, M., und Wright, M. (1986). User's guide for lssol (version 1.0): A fortran package for constrained linear least-squares and convex quadratic programming. Techn. Bericht SOL 86-1, Dept. of Operations Research, Stanford University, Stanford, CA.

Gill, P., Murray, W., und Wright, M. (1992). Practical Optimization. Academic Press, New York.

Goldberg, D. (1989). Genetic Algorithms in Search, Optimization, and MAchine Learning. Addison-Wesley.

Greville, T. (1967). On the normalisation of the B-Splines and the location of the nodes for the case of unequally spaced knots. Academic Press, New York.

Grossman, I. (1990). Mixed-integer nonlinear programming technigues for the synthesis of engineering systems. Research in Engineering Design, 1:205-228.

Grossmann, C. und Terno, J. (1997). Numerik der Optimierung. Teubner Studienbücher.

Hansen, E. (1992). Global optimization using interval analysis. Marcel Dekkar, New York, NY.

Heimann, J. (1993). Seegangskräfte und -bewegungen von optimierten Halbtauchermodellen in transienten Wellenpaketen und irregulärem Seegang. Master's thesis, Technische Universität Berlin. In German.

Hilleary, R. (1966). The Tangent Search Method of constrained minimization. Tech.Rep./Res.Paper 59, United States Naval Postgraduate School, Monterey.

Himmelblau, D. (1972). Applied Nonlinear Programming. McGraw-Hill Book Co., New York.

Himmelblau, D. und Paviani, D. (1969). Constrained nonlinear optimization by heu- 
ristic programming. Operations Research, 17(2).

Hogben, N. und Lumb, F. (1967). Ocean Wave Statistics. Her Majesty's Stationery Office, London.

Holland, J. (1975). Adaptation in natural and artificial systems: an introductory analysis with applications to biology, control, and artificial intelligence. University of Michigan Press, Ann Arbor, MI, USA.

Holland, J. (1992). Adaption in natural and artifical systems. MIT Press, Cambridge, Mass.

Hooke, R. und Jeeves, T. (1961). Direct Search solution of numerical and statistical problems. Journal of the Association for Computing Machinery, 8(4).

Horst, R. und Tuy, H. (1993). Global Optimization: Deterministic Approaches. Springer Verlag, Berlin, 2. edition.

Houck, C., Joines, J., und Kay, M. (1995a). The effective use of local improvement procedures in conjunction with genetic algorithms. Technical reprot ncsu-ie technical report 95, North Carolina State University.

Houck, C., Joines, J., und Kay, M. (1995b). A genetic algorithm for function optimization: A MATLAB implementation. NCSU-IE Technical Report 95-09, North Carolina State University.

Huang, L. (2000). Anwendung von Freiformflächen beim parametergesteuerten Entwurf von Offshore-Plattformen. Dissertation, Institut für Schiffs- und Meerestechnik, Technische Universität Berlin, Germany. In German.

Ingber, L. (1989). Very fast simulated re-annealing. Mathematical and Computer Modelling, 12:967-973.

Ingber, L. (1992). Generic mesoscopic neural networks based on statistical mechanics of neocortical interactions. Physical Review A, 45(4):R2183-R2186.

Ingber, L. (1993). Simulated annealing: practice versus theory. Mathematical and Computer Modelling, 18(11):29-57.

Ingber, L. (1996). Adaptive simulated annealing (ASA): lessons learned. Journal of Control and Cybernetics, 25(1):33-54.

John, F. (1950). On the motion of floating bodies II - Simple harmonic motions. Communications on Pure and Applied Mathematics, 3:45-101.

Johnson, B. (1990). On the integration of CFD and CAD in ship design. In van Oortmerssen, G., editor, Proc. Int. Symp. on CFD and CAD in Ship Design, S. 3-20. Elsevier Science Publishers B.V.

Kagemoto, H. (1992). Minimization of wave forces on an array of floating bodies. Applied Ocean Research, 14(2):83-92.

Kagemoto, H. und Yue, D. (1986). Interactions among multiple three-dimensional bodies in water waves: an exact algebraic method. Journal of Fluid Mechanics, 166:189-209. 
Kearfott, R. (1997). Empirical evaluation of innovations in interval branch and bound algorithms for nonlinear systems. SIAM Journal of Scientific Computing, 18(2):574594.

Kinsman, B. (1965). Wind Waves. Prentice Hall Inc., Englewood Cliffs, New York.

Kirkpatrick, S., Gelatt, Jr., C., und Vecchi, M. (1983). Optimization by Simulated Annealing. Science, 220(4598):671-680.

Kitamura, M. (2001). Genetic algorithm and optimal design of ship structure incorporating dynamic constraints. Class NK TECH BULLETIN 2001.

Klingman, W. und Himmelblau, D. (1964). Nonlinear programming with the aid of a multiple-gradient summation technique. Journal of the Association for Computing Machinery (ACM), 11:400-415.

Kuipers, E. (2000). ftp://ftp.mathworks.com/pub/contrib/v5/optim/bnb/.

Kwik, K. (1969). Zur mathematischen Darstellung von Schiffslinien (on the mathematical representation of ship lines). In Schiffstechnik, Volume Band 16, Heft 83.

Lee, C.-H. und Sclavounos, P. (1989). Removing the irregular frequencies from integral equations in wave body interactions. Journal of Fluid Mechanics, 207:393-418.

Lee, K. Y., Cho, S., und Roh, M. (2002). An efficient global-local hybrid optimization method using design sensitivity analysis. International Journal of Vehicle Design, $28(4): 300-317$.

Longuet-Higgins, M. (1952). On the statistical distribution of the heights of sea waves. Journal of Marine Research, Vol. 9:245-266.

Marcelin, J. (1999). Evolutionary optimisation of mechanical structures: Towards an integrated optimisation. Engineering with Computers, 15:326-333. Springer Verlag London Limited.

Mathisen, J. und Bitner-Gregersen, E. (1990). Joint distributions for significant wave height and wave zero-upcrossing period. Applied Ocean Research, 12(2):93-103.

Matten, R., Str $\phi$ msen, K., Lassabliere, J., und Vogel, H. (1996). The heidrun field: Tlp global performance and hydrodynamics. In Proc. of the Offshore Technology Conference, Houston. OTC 8098.

Mayer, D., Belward, J., und Burrage, K. (1996). Use of advanced techniques to optimize a multi-dimensional diary model. Journal of Agricultural Systems, 50:239-253.

Mei, C. (1989). The applied dynamics of ocean surface waves, Volume 1 von Advanced Series on Ocean Engineering. World Scientific, 2 edition.

Metropolis, N., Rosenbluth, A., Rosenbluth, M., Teller, A., und Teller, E. (1953). Equation of state calculations by fast computing machines. Journal of Chemical Physics, 21(6):1087-1092.

Michalewicz, Z. (1994). Genetic Algorithms + Data Structures = Evolution Programs. AI Series. Springer-Verlag, New York.

Miner, M. (1945). Cumulative damage in fatigue. ASME Journal of Applied Mechanics, 
12:A159-A164.

Mitten, L. (1970). Branch-and-Bound methods: general formulation and properties. Operations Research, 18:24-34.

Morison, J., O'Brian, M., Johnson, J., und Schaaf, S. (1950). The force exerted by surface waves on piles. Pet. Trans. AIME, 189(T.P. 2846):149-157.

Murawski, K., Arciszewski, T., und De Jong, K. (2000). Evolutionary computation in structual design. Enginering with Computers, 16:275-286. Springer Verlag London Limited.

Newman, J. (1977). Marine Hydrodynamics. The MIT Press, Cambridge, Massachusetts.

Newman, J. (1985). Algorithms for the free-surface Green functions. Journal of Engineering Mathematics, 19:57-67.

Newman, J. (1986). Distributions of sources and dipoles over a quadrilateral panel. Journal of Engineering Mathematics, 20:113-126.

Newman, J. und Sclavounos, P. (1988). The computation of wave loads on large offshore structures. In Proc. of Int. Conf. on Behaviour of Offshore Structures (BOSS '88), S. 605-622. Trondheim, Norway.

Nowacki, H. (1994). Rechnergestützter Schiffsentwurf (Computer Aided Ship Design). Institut für Schiffs- und Meerestechnik, Technische Universität Berlin, Germany. Lecture notes (in German).

Nowacki, H. (2003). Design Synthesis and Optimization - A Historical Perspective. In OPTIMISTIC - Optimization in Marine Design, $39^{\text {th }}$ WEGEMT Summer School, Kapitel 1, S. 1-26. Mensch \& Buch Verlag, Berlin.

Nowacki, H., Bloor, M., und Oleksiewicz, B. (1995). Computational geometry for ships. World Scientific Publishers, Singapore, New Jersey, London, Hong Kong.

Nowacki, H. und Reed, A. (1974). Interactive creation of fair ship lines. Journal of Ship Research, 18(2):96-112.

Osyczka, A. (1984). Multicriterion Optimization in Engineering with FORTRAN Programs. Elis Horwood Ltd.

Palmgren, A. (1924). Die Lebensdauer von Kugellagern. VDI, 68. In German.

Papanikolaou, A., Zaraphonitis, G., und Androulakakis, M. (1991). Preliminary design of a high-speed SWATH passenger/car ferry. Marine Technology, 28(3):129-141.

Powell, M. (1970). A survey of numerical methods for unconstrained optimization. SIAM Rev., 12:79-97.

Powell, M. (1971). Recent advances in unconstrained optimization. Methematical Programming, 1:126-57.

Press, W., Teukolsky, S., Vetterling, W., und Flannery, B. (1996). Numerical Recipes in Fortran 90, Volume 2 von The Art of Parallel Scientific Computing. Cambridge University Press, 2 edition. 
Price, W. und Bishop, R. E. D. (1974). Probabilistic Theory of Ship Dynamics. Chapman and Hall, London.

Ratschek, H. und Rokne, J. (1988). New computer methods for global optimization. Mathematics and its Applications. Ellis Horwood Ltd.

Ratz, D. (1995). Globale Optimierung mit Ergebnisverifikation. Techn. Bericht KZFA 5560, Seiten 9-35, Forschungszentrum Karlsruhe.

Rechenberg, I. (1973). Evolutionsstrategie, Optimierung technischer Systeme nach Prinzipien der biologischen Evolution, Volume 15 von Reihe Problemata. F. Frommann Verlag, Stuttgart-Bad Cannstatt.

Rechenberg, I. (1994). Evolutionsstrategie '94. Verlag Frommann-Holzboog.

Rosen, B. (1992). Function optimization based on advanced simulated annealing. In IEEE Workshop on Physics and Computation - PhysComp '92, S. 289-293.

Royo, H. und Sahinidis, N. (1996). A Branch-and-Reduce approach to global optimization. Journal of Global Optimization, 8(2):107-139.

Schittkowski, K. (1985). NLQPL: A FORTRAN-Subroutine Solving Constrained Nonlinear Programming Problems. In Annals of Operations Research, Volume 5, S. 485500 .

Schittkowski, K. (1992). Solving nonlinear programming problems with very many constraints. In Optimization, Volume 25, S. 176-196.

Schoeneburg, E., Heinzmann, F., und Feddersen, S. (1994). Genetische Algorithmen und Evolutionsstrategien. Addison-Wesley, Bonn.

Schwefel, H., Hoffmeister, F., und Bäck, T. (1993). Applications of evolutionary algorithms. Techn. Bericht SYS-2/92, Lehrstuhl für Systemanalyse, Universität Dortmund.

Schwefel, H.-P. (1971). Evolutionary strategie for the numerical optimization. German Science Foundation(DFG) Research Report DFG Ga 24/16, Technische Universität Berlin.

Sen, M. und Stoffa, P. (1995). Global Optimization in Geophysical Inversion. Elsevier, The Netherlands. ISBN 0-444-81767-0.

Söding, H. und Häusler, F.-U. (1976). Minimization of vertical motions of floating structures. In Proc. of BOSS 'r6 Conference, S. 307-319, Trondheim, Norway.

St. Denis, M. und Pierson, W. (1953). On the motions of ships in confused seas. Transactions, SNAME. 61.

Taylor, D. (1915). Caculations for ships' forms and the light thrown by model experiments upon resistance, propulsion and rolling of ships. Transactions International Engineering Congress, (196).

Törn, A. und Zilinskas, A. (1989). Global Optimization. Springer-verlag, Berlin.

van Rossum, G. und Drake Jr., F. L. (2003). An Introduction to Python. Network Theory Ltd. 
Vasconcellos, J. und Vassalos, D. (1991). Preliminary design of semisubmersible platform for floating production system using optimization's techniques. In Proceedings of $1^{\text {th }}$ ISOPE, S. 304-310, Edinburgh, UK. The Int. Soc. of Offshore and Polar Engineers.

WAMIT (1994). WAMIT Version 5.1 - A Radiation-Diffraction Panel Program For Wave-Body Interactions. Userguide, Department of Ocean Engineering, MIT.

Wehausen, J. und Laitone, E. (1960). Surface Waves. Encyclopedia of Physics, Volume IX, pp. 446-778 von Fluid Dynamics III. Springer Verlag Berlin. Edited by S. Flügge.

Weihe, K. (1998). Algorithmen in der Praxis. Konstanzer Schriften in Mathematik und Informatik, Universität Konstanz. ISBN 1430-3558.

Weinblum, G. (1953). Systematische Entwicklung von Schiffsformen (systematic development of hull forms). In Jahrbuch der Schiffbautechnischen Gesellschaft, STG, Volume Band 47, Germany.

Zoutendijk, M. (1960). Methods of Feasible Directions. Elsevier Publ. Co., Amsterdam. 


\section{Anhang A}

\section{Darstellung der Spantarealkurve}

Für die Definition der Spantarealkurve kann der Anwender auf vier unterschiedliche Parametersätze zurückgreifen:

1. $A_{p}, A_{q}, V$ und $\xi_{c}$

2. $A_{p}, A_{q}, V, \xi_{c}$ und $d A /\left.d \xi\right|_{p}$

3. $A_{p}, A_{q}, V, \xi_{c}$ und $d A /\left.d \xi\right|_{q}$

4. $A_{p}, A_{q}, V, \xi_{c}, d A /\left.d \xi\right|_{p}$ und und $d A /\left.d \xi\right|_{q}$

Für die Spantarealkurve $A(\xi)$ mit $\xi \in[0, L]$ wird der Ansatz

$$
A(\xi)=\pi r^{2}(\xi)
$$

gemacht, wobei die Radiusverteilung $r(\xi)$ durch eine B-Spline-Kurve repräsentiert wird.

$$
\begin{aligned}
r(\xi)=r(t L) & =\sum_{j=0}^{m-1} P_{j} B_{j, k}(t) \\
\text { mit } \quad \xi & =t L, t \in[0,1] .
\end{aligned}
$$

Die $B_{j, k}(t)$ sind die B-Spline-Basis-Polynome der Ordnung $k$ mit einem Knotenvektor $T$. Der Knotenvektor $T$ enthält $m+k$ Elemente in folgender Form:

$$
T=\left(t_{0}, t_{1}, t_{2}, \cdots t_{m+k-3}, t_{m+k-2}, t_{m+k-1}\right)
$$

wobei den ersten und den letzten $k$ Elementen der gleiche Zahlenwert (jeweils 0 und 1) zugeordnet wird. Man kann z.B. innere Knoten äquidistant setzen. Werden die Zwischenknoten mit:

$$
T_{j}=\frac{1}{k}\left(t_{j+1}+\cdots+t_{j+k}\right)
$$

eingeführt und die Kontrollpunkte $P_{j}$ über $T_{j}$ aufgetragen, erhält man eine B-SplineKurve über der $t$-Achse. Entsprechend der Spantarealkurve $A(\xi)$ bezeichnet man $r(\xi)$ 
als Spantradienkurve oder Radienkurve. Abb. A.1 zeigt eine B-Spline-Kurve über der $t$-Achse. Diese parametrische Kurve lässt sich wie folgt darstellen:

$$
\left(\begin{array}{c}
r(t L) \\
t
\end{array}\right)=\left(\begin{array}{l}
\sum_{j=0}^{m-1} P_{j} B_{j, k}(t) \\
\sum_{j=0}^{m-1} T_{j} B_{j, k}(t)
\end{array}\right)=\sum_{j=0}^{m-1}\left(\begin{array}{c}
P_{j} \\
T_{j}
\end{array}\right) B_{j, k}(t)
$$

wobei die Greville-Identität (Greville, 1967) eingesetzt wird:

$$
t=\sum_{j=0}^{m-1} T_{j} B_{j, k}(t)
$$

Gesucht sind in Gl. A.5 die Kontrollpunkte $P_{j}$. Sie werden durch Interpolation aus den vorgegebenen Parametern bestimmt (Huang, 2000). Für den 4. Parametersatz lautet z.B. das Interpolationsproblem:

$$
\begin{gathered}
A_{p}=\sum_{i=0}^{m-1} \sum_{j=0}^{m-1} P_{i} P_{j} B_{i, k}(0) B_{j, k}(0) \\
A_{q}=\sum_{i=0}^{m-1} \sum_{j=0}^{m-1} P_{i} P_{j} B_{i, k}(1) B_{j, k}(1) \\
V=\pi L \int_{0}^{1} \sum_{i=0}^{m-1} \sum_{j=0}^{m-1} P_{i} P_{j} B_{i, k}(t) B_{j, k}(t) d t \\
\xi_{c}=\frac{\pi L}{V} \int_{0}^{1} t \sum_{i=0}^{m-1} \sum_{j=0}^{m-1} P_{i} P_{j} B_{i, k}(t) B_{j, k}(t) d t \\
\left.\frac{d A}{d \xi}\right|_{p}=\frac{2 \pi}{L} \sum_{i=0}^{m-1} \sum_{j=0}^{m-1} P_{i} P_{j} B_{i, k}(0) B_{j, k}^{\prime}(0) d t \\
\left.\frac{d A}{d \xi}\right|_{q}=\frac{2 \pi}{L} \sum_{i=0}^{m-1} \sum_{j=0}^{m-1} P_{i} P_{j} B_{i, k}(1) B_{j, k}^{\prime}(1) d t
\end{gathered}
$$

wobei $B_{j, k}^{\prime}(t)$ die Ableitung der B-Spline-Basisfunktionen bezüglich $t$ ist. Da für den 4. Parametersatz 6 Gleichungen bekannt sind, muss die Anzahl der Kontrollpunkte $m$ den Wert 6 annehmen, um das gekoppelte nichtlineare Gleichungssystem (A.7) eindeutig lösen zu können. Für die Formvariation wird die Ordnung der B-Splinefunktionen $B_{j, k}(t)$ quadratisch $(k=3)$ gehalten. Bei höheren Ordnungen schwingt die B-SplineKurve sehr stark und es können leicht entartete Kurven (z.B. Kurven mit Überlappungen) entstehen. Für die quadratischen B-Splinefunktionen fallen die Randknoten 3-fach zusammen. Daraus lassen sich die ersten beiden Gleichungen von (A.7) wie folgt vereinfachen:

$$
\begin{gathered}
A_{p}=\pi P_{0}^{2} \\
A_{q}=\pi P_{m-1}^{2}
\end{gathered}
$$

Die Anzahl der Unbekannten $P_{j}$ reduziert sich von $m$ auf $m-2$. Die übrigen Kontrollpunkte können durch Lösen des nichtlinearen Gleichungssystems mit Hilfe eines Newton-Verfahrens berechnet werden. Das gewählte Verfahren nach Press et al. (1996) 


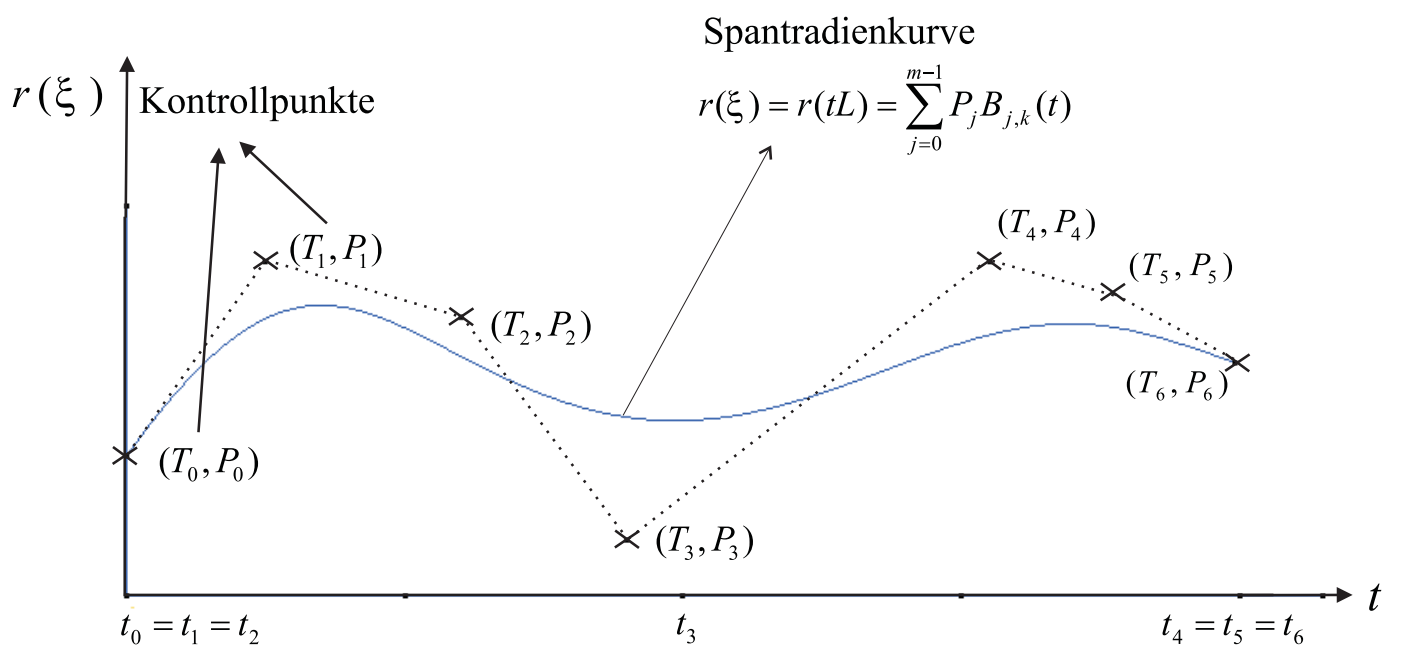

Abbildung A.1: Quadratischer B-Spline

ist sehr robust und konvergiert für fast beliebige Anfangsvektoren. Sind die $P_{j}$ bekannt, ist die Radienkurve $r(\xi)$ durch Gl. A.5 gegeben. Für die Spantarealkurve gilt dann entsprechend:

$$
\left(\begin{array}{c}
A(\xi) \\
\xi
\end{array}\right)=\left(\begin{array}{c}
\pi\left(\sum_{j=0}^{m-1} P_{j} B_{j, k}(t)\right)^{2} \\
L \sum_{j=0}^{m-1} T_{j} B_{j, k}(t)
\end{array}\right) \quad \text { mit } \quad \xi=\mathrm{tL}, \mathrm{t} \in[0,1]
$$




\section{Anhang B}

\section{Grundlagen des TFC-Programmes}

Zur Berechnung der Bewegungsübertragungsfunktion für hydrodynamisch transparente Strukturen wurde frühere Arbeit von Birk (1990a) um die zähigkeitsbedingten Kraftund Dämpfungsanteile ergänzt und auf veränderliche Querschnittsgrößen erweitert. Im folgenden werden theoretische Grundlagen des erweiterten Programms TFC (Transfer

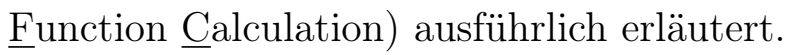

\section{B.1 Hydrodynamisch transparente Strukturen}

In der Praxis wird eine Struktur als hydrodynamisch transparent angesehen, wenn für das Verhältnis einer charakteristischen Bauteilgröße (z.B. der Durchmesser $D$ einer Säule) und der Wellenlänge $\lambda$ gilt:

$$
D / \lambda<0.2
$$

In diesem Fall passiert die Welle die Struktur ohne merkliche Störung. Beispiele für solche Konstruktionen sind kleine und mittelgroße Halbtaucher, sowie die Rohrkonstruktionen feststehender Jackets. Bei hydrodynamisch transparenten Konstruktionen sind Trägheits- und Widerstandskräfte maßgebend, während bei großvolumigen, kompakten Konstruktionen Trägheits- und Diffraktionseffekte dominieren.

Mit vereinfachenden Annahmen für schlanke Zylinder haben Morison et al. (1950) eine Gleichung für die Horizontalkraft auf die Mantelfläche senkrechter Pfähle entwickelt (Morisongleichung), die von Chakrabarti et al. (1975) für im Raum beliebig orientierte, feststehende Zylinder erweitert wurde. Zur Berechnung der hydrodynamischen Kräfte auf beliebig angeordnete und bewegte zylindrische Bauteile im Seegang wird hier die erweiterte Form der Morisongleichung verwendet (Clauss et al., 1992).

\section{B.2 Beschreibung der Struktur}

Für die Berechnung der Kräfte wird die Struktur in einzelne Kreiszylinder mit veränderlichem Durchmesser aufgeteilt. Die Endflächen sollen senkrecht zur Zylinderachse stehen. Durch Aufspalten der Struktur in einzelne Teile entstehen bei der rechnerinternen Darstellung zusätzliche benetzte Flächen, z.B. die untere Endfläche der Säule 
und die entsprechende Mantelfläche des Auftriebskörpers. Die zusätzlichen Kräfte sind jedoch entgegengesetzt und heben sich wieder auf. Da das Verhältnis der Wellenlänge zum Zylinderdurchmesser $(\lambda / D)$ groß ist, wird angenommen, dass sich durch die komplizierten Verbindungsbereiche keine signifikanten Unterschiede für die Erregerkaft auf die Gesamtstruktur ergeben.

\section{B.2.1 Koordinatensystem}

Zur Beschreibung der Geometrie wird ein kartesisches Koordinatensystem mit der Basis $\left\langle\mathbf{e}_{x}, \mathbf{e}_{x}, \mathbf{e}_{z}\right\rangle$ eingesetzt (siehe Abb. B.1). Der Ursprung 0 soll in der Ruhewasserlinie
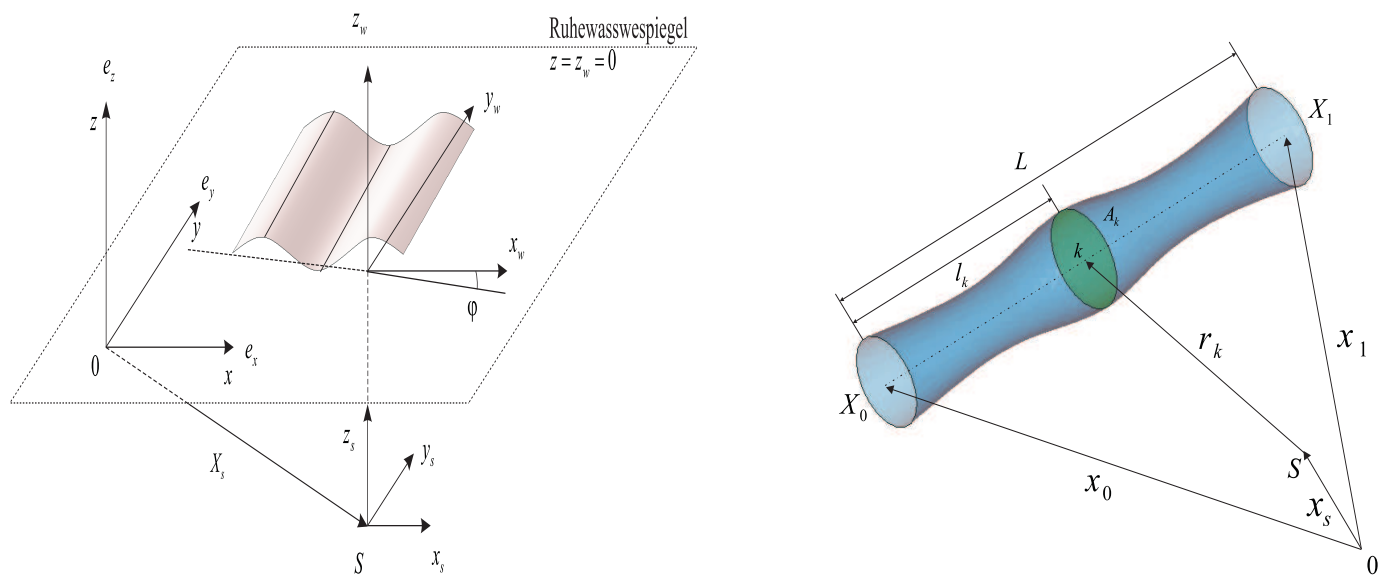

Abbildung B.1: Koordinatensysteme und Beschreibung eines Strukturelements

liegen. Das Koordinatensystem $\left(x_{w}, y_{w}, z_{w}\right)$ für die langkämmigen Wellen hat seinen Ursprung ebenfalls in der Ruhewasserlinie, aber senkrecht über dem Gewichtsschwerpunkt $S$. Um unterschiedliche Einfallsrichtungen der Wellen zu berücksichtigen, kann das Wellenkoordinatensystem um den Winkel $\varphi$ in der $x-y$ Ebene gedreht sein. Bei beiden Koordinatensystemen zeigen die z-Achsen nach oben.

Der Vektor vom Ursprung 0 zum Schwerpunkt $S$ wird mit $\mathbf{x}_{\mathbf{s}}$ bezeichnet. Die Position eines kreiszylindrischen Strukturelements im Raum wird durch den Anfangspunkt $X_{0}$, mit Ortsvektor $\mathbf{x}_{0}$ und den Endpunkt $X_{1}\left(\mathbf{x}_{1}\right)$ festgelegt. Die Länge $L$ des Zylinders ergibt sich aus dem Betrag des Vektors $\mathbf{x}_{\mathbf{1}}-\mathbf{x}_{\mathbf{0}}$ :

$$
L=\left|\mathbf{x}_{1}-\mathbf{x}_{0}\right|
$$

Der Einheitsvektor $\mathbf{e}_{T}$ in Längsrichtung des Zylinders zeigt immer von $\mathbf{x}_{0}$ nach $\mathbf{x}_{1}$ :

$$
\mathbf{e}_{T}=\left(\mathbf{x}_{1}-\mathbf{x}_{0}\right) / L
$$

Ein Punkt $k$ auf dem Zylinder lässt sich folglich durch den Ortsvektor $\mathbf{r}$ (vom Ursprung aus)

$$
\mathbf{r}_{0}=\mathbf{x}_{0}+\ell_{k} \mathbf{e}_{T} \quad \text { mit } \quad 0<\ell_{k}<L
$$


oder, bezogen auf den Schwerpunkt $S$, durch

$$
\mathbf{r}_{S}=\mathbf{x}_{0}-\mathbf{x}_{s}+\ell_{k} \mathbf{e}_{T} \quad \text { mit } \quad 0<\ell_{k}<L
$$

beschreiben. Da das Wellenkoordinatensystem aus dem der Geometrie durch eine Verschiebung und eine Drehung um den Winkel $\varphi$ in der x-y-Ebene hervorgeht, gilt für den Vektor $\mathbf{r}_{w}$ die einfache Beziehung:

$$
\mathbf{r}_{w}=\left[\begin{array}{ccc}
\cos \varphi & \sin \varphi & 0 \\
-\sin \varphi & \cos \varphi & 0 \\
0 & 0 & 1
\end{array}\right] \cdot\left(\mathbf{r}_{0}-\left(\begin{array}{c}
x_{s x} \\
x_{s y} \\
0
\end{array}\right)\right)
$$

Ausgeschrieben lautet Gleichung (B.4) demnach:

$$
\mathbf{r}_{w}=\left(\begin{array}{c}
x_{w} \\
y_{w} \\
z_{w}
\end{array}\right)=\left(\begin{array}{c}
\left(x_{0 x}-x_{s x}+\ell_{k} e_{T x}\right) \cos \varphi+\left(x_{0 y}-x_{s y}+\ell_{k} e_{T y}\right) \sin \varphi \\
-\left(x_{0 x}-x_{s x}+\ell_{k} e_{T x}\right) \sin \varphi+\left(x_{0 y}-x_{s y}+\ell_{k} e_{T y}\right) \cos \varphi \\
x_{0 z}+\ell_{k} e_{T z}
\end{array}\right)
$$

\section{B.2.2 Bewegungsfreiheitsgrade}

Ein frei schwimmender starrer Körper besitzt im Raum 6 Freiheitsgrade. Abb. B.2 zeigt die drei translatorischen Freiheitsgrade $s_{1}, s_{2}, s_{3}$ (Verschiebungen) und die drei rotatorischen $s_{4}, s_{5}, s_{6}$ (Drehungen). Zur einfachen Darstellung der Trägheitskraft $\mathbf{F}$ bzw. des

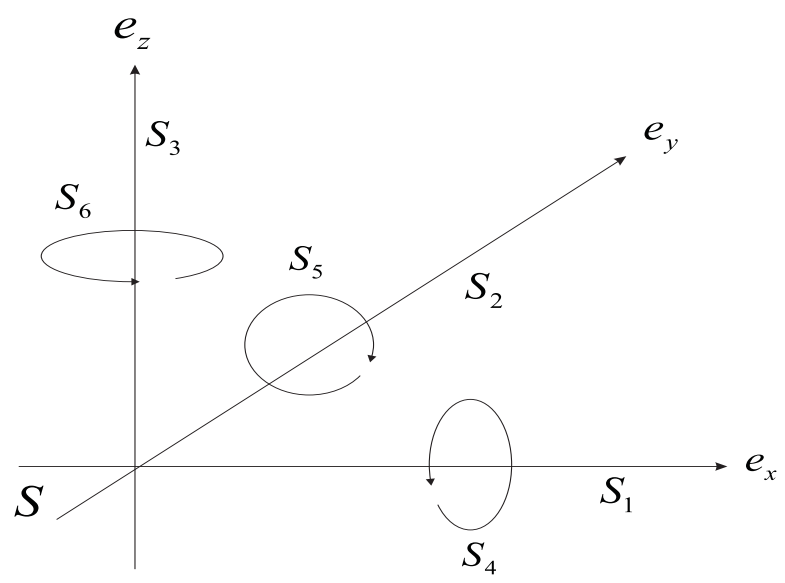

$s_{1}$ Horizontalbewegung (surge)

$s_{2}$ Querbewegung (sway)

$s_{3}$ Vertikalbewegung (heave)

$s_{4}$ Rollbewegung (roll)

$s_{5}$ Stampfbewegung (pitch)

$s_{6}$ Gierbewegung (yaw)

Abbildung B.2: Bewegungsfreiheitsgrade

Momentes $\mathbf{M}$ des Körpers sollen die Strukturbewegungen auf den Massenschwerpunkt des Systems bezogen werden. Die Verschiebungen $s_{1}, s_{2}$ und $s_{3}$ des Schwerpunkte $S$ in Richtung der Koordinatenachsen ergeben den Verschiebungsvektor:

$$
\mathbf{S}_{T}=\left(\begin{array}{c}
s_{1} \\
s_{2} \\
s_{3}
\end{array}\right)
$$


Die Drehungen $s_{4}, s_{5}$ und $s_{6}$ um die Achsen $x_{s}, y_{s}, z_{s}$ durch den Schwerpunkt bilden den Drehvektor:

$$
\mathbf{s}_{D}=\left(\begin{array}{c}
s_{4} \\
s_{5} \\
s_{6}
\end{array}\right)
$$

Um die Schreibweise zu vereinfachen werden die beiden Vektoren in einer Spaltenmatrix zusammengefasst:

$$
\mathbf{s}^{*}=\left(\begin{array}{c}
\mathbf{s}_{T} \\
\mathbf{s}_{D}
\end{array}\right)
$$

Der verallgemeinerte Bewegungsvektor $\mathbf{s}^{*}$ ist kein physikalischer Vektor, da er Größen verschiedener Dimension enthält. Deshalb wird die Größe zusätzlich durch das Zeichen * gekennzeichnet. Diese Schreibweise wird im folgenden auch für die Darstellung des verallgemeinerten Kraftvektors verwendet.

\section{B.3 Berechnung der Kräfte auf ein Strukturelement}

Die äußere Kraft (Moment) auf ein Strukturelement resultiert aus der durch Seegang und Strukturbewegung erzeugten Strömung. Die Gewichts- und die Auftriebskraft der Ruhelage ergeben keinen Beitrag zum dynamischen Kräftegleichgewicht, da sie als Resultierende den Nullvektor haben.

Der Teil der äußeren Kraft, der durch Beschleunigung (bzw. Geschwindigkeit) von Wasserpartikeln und der Struktur verursacht wird, bezeichnet man als hydrodynamische Kraft. Durch Auslenkung der schwimmenden Struktur aus der Ruhelage verändert sich die resultierende Auftriebskraft. Die Differenz zwischen veränderter Auftriebskraft und Gewichtskraft ist die sogenannte hydrostatische Rückstellkraft.

Die hydrodynamische Kraft wird bei beliebigen Körpern durch die Integration des instationären Druckes über die benetzte Oberfläche gewonnen. Bei einem zylindrischen Körper ist es zweckmäßig, die Oberfläche in die Mantelfläche und die Deckelflächen des Zylinders aufzuteilen und die Kraftanteile getrennt zu ermitteln. Da sich der Durchmesser der Querschnittsfläche ändern kann, wirken auf die Mantelfläche jedoch neben der normal zur Zylinderachse gerichteten Kräfte auch Kräfte in Richtung der Zylinderlängsachse. Bei der Berechnung der hydrodynamischen Kraft werden deshalb die zusätzlichen Kraftanteile in Längsrichtung des Zylinders berücksichtigt.

\section{B.3.1 Kraft normal zur Zylinderachse}

Für beliebig orientierte, bewegte Zylinder ergibt die Morisongleichung für die normal zur Zylinderachse gerichtete Kraft auf einen Streifen $d \ell$ des Mantels (siehe Abb. B.3):

$$
d \mathbf{F}_{N}=\rho \mathrm{A} \frac{\partial \mathbf{v}_{N}}{\partial t} d \ell+C_{a} \rho \mathrm{A} \frac{\partial \mathbf{u}_{R N}}{\partial t} d \ell+\frac{1}{2} C_{d} \rho \mathrm{D}\left|\mathbf{u}_{R N}\right| \mathbf{u}_{R N} d \ell
$$

Der Kraftvektor $d \mathbf{F}_{N}$ setzt sich aus drei Anteilen zusammen: 


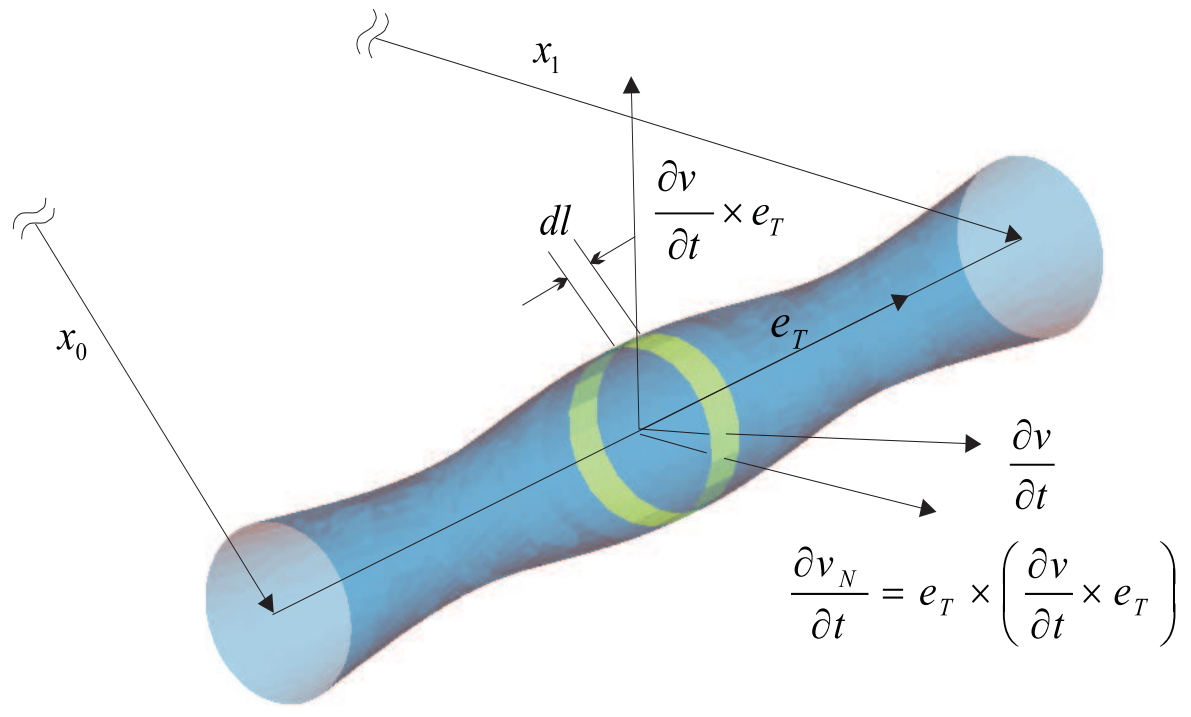

Abbildung B.3: Wellenpartikelbeschleunigung am kreiszylindrischen Strukturelement mit veränderlichen Querschnittsgrößen

- Froude-Krylov-Kraft $\mathbf{F}_{F K N}$ : Sie ist die Wirkung des ungestörten Druckfeldes der beschleunigten Außenströmung und ist proportional der normal zur Zylinderachse gerichteten Komponente $\partial \mathbf{v}_{N} / \partial t$ der Wellenpartikelbeschleunigung $\partial \mathbf{v} / \partial t$. Entsprechend den Annahmen für hydrodynamisch transparente Strukturen wird für $\partial \mathbf{v} / \partial t$ der Wert am Ort der Zylinderachse eingesetzt (siehe Abb. B.3).

- Hydrodynamische Massenkraft $\mathbf{F}_{M a N}$ : Sie ist proportional zur Normalkomponente der Relativbeschleunigung $\partial \mathbf{u}_{R} / \partial t$ zwischen Körper und Fluid. Der hydrodynamische Koeffizient $C_{a}$ ist von mehreren Faktoren (z.B. Körperform, Form der Orbitalbahn, Bodeneffekt bei begrenzter Wassertiefe) abhängig. Potentialtheoretisch ergibt sich für einen Kreiszylinder in unbegrenzter Flüssigkeit der Wert $C_{a}=1.0$ (Clauss et al., 1992). Da Kreiszylinder quer zu ihrer Achse keine ausgezeichnete Bewegungsrichtung besitzen, ist $C_{a}$ davon unabhängig und tritt als skalarer Wert auf.

- Widerstandskraft $\mathbf{F}_{D N}$ : Der 3.Term beschreibt die zähigkeitsbedingte Widerstandskraft. Sie ist proportional zum Quadrat der Relativgeschwindigkeit und daher nichtlinear. Der Widerstandsbeiwert $C_{d}$ ist wie $C_{a}$ von mehreren Faktoren (vor allem der Reynoldszahl und der Rauigkeit) abhängig und muss experimentell ermittelt werden. Für hydrodynamisch transparenten Strukturen spielt die zähigkeitsbedingte Kraft bei hohen Wellen und größeren Wellenperioden eine Rolle, wobei die Zähigkeitsdämpfung beim Bewegungsverhalten vor allem im Bereich der Resonanzfrequenzen und Auslöschungsstellen berücksichtigt werden muss. 
Um die Ermittlung der Übertragungsfunktion linear durchzuführen, muss die nichtlineare Widerstandskraft linearisiert werden. Die Linearisierung erfolgt durch Reihenentwicklung bzw. Energieäquivalenz (Clauss et al., 1992). Hieraus ergibt sich der Ausdruck für die linearisierte Widerstandskraft:

$$
\begin{aligned}
d \mathbf{F}_{D N} & =\frac{\rho}{2} \frac{8}{3 \pi} C_{d} \mathrm{D}\left[\mathbf{u}_{R N}\right]_{a} \mathbf{u}_{R N} d \ell=\frac{\rho}{2} C_{d \ell} \mathrm{D} \mathbf{u}_{R N} d \ell \\
\text { mit } \quad C_{d \ell} & =\frac{8}{3 \pi} C_{d} \mathrm{D}\left[\mathbf{u}_{R N}\right]_{a}
\end{aligned}
$$

Dabei bezeichnet $\left[\mathbf{u}_{R N}\right]_{a}$ die Amplitude der Relativgeschwindigkeit. Damit gilt für ein differentielles zylindrisches Element die linearisierte Morisongleichung:

$$
\begin{aligned}
d \mathbf{F} & =\rho \mathrm{A} \frac{\partial \mathbf{v}_{N}}{\partial t} d \ell+C_{a} \rho \mathrm{A} \frac{\partial \mathbf{u}_{R N}}{\partial t} d \ell+\frac{\rho}{2} C_{d \ell} \mathrm{D} \mathbf{u}_{R N} d \ell \\
& =d \mathbf{F}_{F K N}+d \mathbf{F}_{M a N}+d \mathbf{F}_{D N}
\end{aligned}
$$

Die Kraft bzw. das Moment des hydrodynamischen Druckes auf den Zylindermantel wird nun durch Integration von Gleichung B.10 über die Zylinderlänge ermittelt:

$$
\mathbf{F}_{N}^{*}=\int_{\ell}\left(\begin{array}{c}
d \mathbf{F}_{F K N} \\
\mathbf{r} \times d \mathbf{F}_{F K N}
\end{array}\right)+\int_{\ell}\left(\begin{array}{c}
d \mathbf{F}_{M a N} \\
\mathbf{r} \times d \mathbf{F}_{M a N}
\end{array}\right)+\int_{\ell}\left(\begin{array}{c}
d \mathbf{F}_{D N} \\
\mathbf{r} \times d \mathbf{F}_{D N}
\end{array}\right)
$$

Im folgenden werden die drei Kraftanteile einzeln behandelt. Die auftretenden Terme werden zweckmäßig umgeformt und die unbekannten Bewegungsgrößen von gegebenen geometrischen Größen getrennt. Letztere können dann in Matrizenschreibweise zusammengefasst werden.

\section{Froude-Krylov-Kraft normal zur Zylinderachse}

Aus Gl. (B.11) folgt für die Froude-Krylov-Kraft, die normal zur Zylinderachse auf die Mantelfläche wirkt:

$$
\mathbf{F}_{F K N}=\int_{0}^{L} \rho \mathrm{A} \frac{\partial \mathbf{v}_{N}}{\partial t} d \ell
$$

Mit dem Einheitsvektor in Richtung der Zylinderachse $\mathbf{e}_{T}=\left(e_{T x}, e_{T y}, e_{T z}\right)^{T}$ erhält man $\partial \mathbf{v}_{N} / \partial t$ aus dem doppelten Kreuzprodukt von $\mathbf{e}_{T}$ und der Partikelbeschleunigung $\partial \mathbf{v} / \partial t($ Abb. B.3):

$$
\frac{\partial \mathbf{v}_{N}}{\partial t}=\mathbf{e}_{T} \times\left(\frac{\partial \mathbf{v}}{\partial t} \times \mathbf{e}_{T}\right)
$$

Um $\partial \mathbf{v} / \partial t$ aus dem Ausdruck herausziehen zu können, wird das Kreuzprodukt durch äquivalente Matrizenprodukte ${ }^{1}$ ersetzt:

$$
\frac{\partial \mathbf{v}_{N}}{\partial t}=\frac{\partial\left(\mathbf{T}_{N} \mathbf{v}\right)}{\partial t}
$$

\footnotetext{
${ }^{1}$ Das Kreuzprodukt $(\mathbf{a} \times \mathbf{b})$ zweier Vektoren $\mathbf{a}=\left(a_{1}, a_{2}, a_{3}\right)^{T}$ und $\mathbf{b}=\left(b_{1}, b_{2}, b_{3}\right)^{T}$ lässt sich durch die folgende Matrizenoperation ersetzen:

$\mathbf{a} \times \mathbf{b}=\left[\begin{array}{ccc}0 & b_{3} & -b_{2} \\ -b_{3} & 0 & b_{1} \\ b_{2} & -b_{1} & 0\end{array}\right] \mathbf{a}$

wie sich leicht durch Nachrechnen zeigen lässt.
} 
$\mathbf{T}_{N}$ ist dabei die folgende Transformationsmatrix:

$$
\mathbf{T}_{N}=\left[\begin{array}{ccc}
1-e_{T x}^{2} & -e_{T x} e_{T y} & -e_{T x} e_{T z} \\
-e_{T y} e_{T x} & 1-e_{T y}^{2} & -e_{T y} e_{T z} \\
-e_{T z} e_{T x} & -e_{T z} e_{T y} & 1-e_{T z}^{2}
\end{array}\right]
$$

Hier sollen nur gerade Bauteile betrachtet werden. Deshalb kann die Transformationsmatrix $\mathbf{T}_{N}$ aus dem Integral herausgezogen werden, da $\mathbf{T}_{N}$ (bzw. $\mathbf{e}_{T}$ ) unabhängig von der Integrationsvariablen $\ell$ ist. Setzt man Gl. (B.13) in Gl. (B.12) ein, folgt:

$$
\mathbf{F}_{F K N}=\rho \mathbf{T}_{N} \int_{0}^{L} \mathrm{~A}(\ell) \frac{\partial \mathbf{v}}{\partial t} d \ell
$$

Da hier der Querschnitt $A$ veränderlich sein kann, hängt er von der Integrationsvariablen $\ell$ ab. Bei der Integration wird für den veränderlichen Querschnitt $A$ die Spantarealkurve (siehe Gl. A.9) eingesetzt, die aus gegebenen Formparametern mit Hilfe der B-Spline-Kurve bestimmt worden ist.

Für das Moment $\mathbf{M}_{F K N}$ der Froude-Krylov-Kraft ergibt sich mit Gl. (B.13) aus Gl. (B.11):

$$
\mathbf{M}_{F K N}=\int_{0}^{L}\left[\mathbf{r} \times \rho \mathrm{AT}_{\mathbf{N}} \frac{\partial \mathbf{v}}{\partial \mathbf{t}}\right] d \ell
$$

Setzt man für den Ortsvektor r Gl. (B.2) in Gl. (B.16) ein, kann man das Integral zweckmäßig in folgender Weise aufspalten:

$$
\mathbf{M}_{F K N}=\int_{0}^{L}\left[\left(\mathbf{x}_{0}-\mathbf{x}_{s}\right) \times \rho \mathrm{A} \mathbf{T}_{N} \frac{\partial \mathbf{v}}{\partial t}\right] d \ell+\int_{0}^{L}\left[\mathbf{e}_{T} \times \rho \mathrm{A} \mathbf{T}_{N} \ell \frac{\partial \mathbf{v}}{\partial t}\right] d \ell
$$

Die Kreuzprodukte können auch analog zu Gl. (B.13) durch folgende Matrizen ersetzt werden:

$$
\begin{aligned}
\mathbf{X}_{0 s}= & {\left[\begin{array}{ccc}
0 & -x_{0 z}+x_{s z} & x_{0 y}-x_{s y} \\
x_{0 z}-x_{s z} & 0 & -x_{0 x}+x_{s x} \\
-x_{0 y}+x_{s y} & x_{0 x}-x_{s x} & 0
\end{array}\right] } \\
\mathbf{E}_{T}= & {\left[\begin{array}{ccc}
0 & -e_{T z} & e_{T y} \\
e_{T z} & 0 & -e_{T x} \\
-e_{T y} & e_{T x} & 0
\end{array}\right] }
\end{aligned}
$$

Damit folgt aus Gl. (B.17):

$$
\mathbf{M}_{F K N}=\rho \mathbf{X}_{0 s} \mathbf{T}_{N} \int_{0}^{L} \mathrm{~A} \frac{\partial \mathbf{v}}{\partial t} d \ell+\rho \mathbf{E}_{T} \mathbf{T}_{N} \int_{0}^{L} \mathrm{~A} \ell \frac{\partial \mathbf{v}}{\partial t} d \ell
$$


Führt man für die beiden verbleibenden, unbekannten Integrale die Abkürzungen

$$
\mathbf{I}_{A v}=\int_{0}^{L} \mathrm{~A} \frac{\partial \mathbf{v}}{\partial t} d \ell \quad \text { und } \quad \mathbf{I}_{A v l}=\int_{0}^{L} \mathrm{~A} \ell \frac{\partial \mathbf{v}}{\partial t} d \ell
$$

ein, lässt sich $\mathbf{M}_{F K N}$ in Form von Matrizen darstellen:

$$
\mathbf{M}_{F K N}=\rho\left[\begin{array}{ll}
\mathbf{X}_{0 s} \mathbf{T}_{N} & \mathbf{E}_{T} \mathbf{T}_{N}
\end{array}\right]\left[\begin{array}{c}
\mathbf{I}_{A v} \\
\mathbf{I}_{A v l}
\end{array}\right]
$$

Abschließend lassen sich die Gleichungen (B.15) und (B.21) zu dem verallgemeinerten Kraftvektor $\mathbf{F}_{F K N}^{*}$ in einer Spaltenmatrix zusammenfassen:

$$
\mathbf{F}_{F K N}^{*}=\rho\left[\begin{array}{cc}
\mathbf{T}_{N} & \mathbf{0} \\
\mathbf{X}_{0 s} \mathbf{T}_{N} & \mathbf{E}_{T} \mathbf{T}_{N}
\end{array}\right]\left[\begin{array}{c}
\mathbf{I}_{A v} \\
\mathbf{I}_{A v l}
\end{array}\right]
$$

\section{Hydrodynamische Massenkraft normal zur Zylinderachse}

Für die hydrodynamische Massenkraft $\mathbf{F}_{M a N}$ auf die Mantelfläche (normal zur Zylinderachse) ergibt der zweite Term von Gl. (B.11):

$$
\mathbf{F}_{M a N}=\int_{0}^{L} C_{a} \rho \mathrm{A}(\ell) \frac{\partial \mathbf{u}_{R N}}{\partial t} d \ell
$$

Die Relativgeschwindigkeit $\mathbf{u}_{R}$ erhält man aus der Differenz von Wellenpartikelgeschwindigkeit $\mathbf{v}$ und der Körpergeschwindigkeit $\mathbf{u}_{k}$ :

$$
\mathbf{u}_{R}=\mathbf{v}-\mathbf{u}_{k}
$$

Letztere ist über die Eulersche Geschwindigkeitsformel für starre Körper durch Translationsund Rotationsgeschwindigkeit $\mathbf{u}_{T}$ und $\mathbf{u}_{D}$ des Schwerpunktes gegeben. Vernachlässigt man beim Differenzieren der Geschwindigkeit die Zentripetalbeschleunigung (sie ist von 2. Ordnung), ergibt sich für $\partial \mathbf{u}_{R} / \partial t$ :

$$
\frac{\partial \mathbf{u}_{R}}{\partial t}=\frac{\partial \mathbf{v}}{\partial t}-\frac{\partial \mathbf{u}_{T}}{\partial t}-\frac{\partial \mathbf{u}_{D}}{\partial t} \times \mathbf{r}
$$

Dabei wird für $\mathbf{r}$ der Ortsvektor vom Schwerpunkt $S$ zum Punkt $K$ (Gl. B.2) eingesetzt. Analog zu $\partial \mathbf{v}_{N} / \partial t$ (Gl. B.13) erhält man die Normalkomponente der Relativbeschleunigung mit der Transformationsmatrix $\mathbf{T}_{N}$ nach Gl. (B.14):

$$
\frac{\partial \mathbf{u}_{R N}}{\partial t}=\mathbf{T}_{N} \frac{\partial \mathbf{u}_{R}}{\partial t}=\mathbf{T}_{N}\left(\frac{\partial \mathbf{v}}{\partial t}-\frac{\partial \mathbf{u}_{T}}{\partial t}-\frac{\partial \mathbf{u}_{D}}{\partial t} \times \mathbf{r}\right)
$$

Wird Gl. (B.26) in Gl. (B.23) für $\mathbf{F}_{M a N}$ eingesetzt, folgt:

$$
\mathbf{F}_{M a N}=\rho \mathbf{T}_{N} \int_{0}^{L} C_{a} A(\ell)\left[\frac{\partial \mathbf{v}}{\partial t}-\frac{\partial \mathbf{u}_{T}}{\partial t}-\frac{\partial \mathbf{u}_{D}}{\partial t} \times \mathbf{r}\right] d \ell
$$


Der hydrodynamische Massenbeiwert $C_{a}$ wird als konstant über die Länge $L$ angenommen, oder ist zumindest durch einen Mittelwert ersetzbar. Damit folgt aus Gl. (B.27):

$$
\mathbf{F}_{M a N}=C_{a} \rho \mathbf{T}_{N}\left[\int_{0}^{L} A(\ell) \frac{\partial \mathbf{v}}{\partial t} d \ell-\frac{\partial \mathbf{u}_{T}}{\partial t} \int_{0}^{L} A(\ell) d \ell-\frac{\partial \mathbf{u}_{D}}{\partial t} \times \int_{0}^{L} A(\ell) \mathbf{r} d \ell\right]
$$

Die Beschleunigungsvektoren des Schwerpunktes $\partial \mathbf{u}_{T} / \partial t$ und $\partial \mathbf{u}_{D} / \partial t$ sind von der Integrationsvariablen $\ell$ unabhängig und werden deshalb aus dem Integral herausgezogen.

Um die unbekannten Vektoren aus der eckigen Klammer herausziehen zu können, wird das Kreuzprodukt $\partial \mathbf{u}_{D} / \partial t \times \int_{0}^{L} A(\ell) \mathbf{r} d \ell$ zweckmäßig umgeformt und wieder durch ein äquivalentes Matrizenprodukt ersetzt:

$$
\mathbf{F}_{M a N}=C_{a} \rho \mathbf{T}_{N} \int_{0}^{L} A(\ell) \frac{\partial \mathbf{v}}{\partial t} d \ell-C_{a} \rho V \mathbf{T}_{N}\left[\frac{\partial \mathbf{u}_{T}}{\partial t}-\left(\mathbf{X}_{0 s}+\frac{I_{A \ell}}{V} \mathbf{E}_{T}\right) \frac{\partial \mathbf{u}_{D}}{\partial t}\right]
$$

mit den Abkürzungen:

$$
V=\int_{0}^{L} A(\ell) d \ell \quad \text { und } \quad I_{A \ell}=\int_{0}^{L} A(\ell) \ell d \ell
$$

Der erste Term in dieser Gleichung ist das $C_{a}$-fache der schon bekannten Froude-KrylovKraft (Gl. B.15). Werden die Vektoren $\partial \mathbf{u}_{T} / \partial t$ und $\partial \mathbf{u}_{D} / \partial t$ zu einer Spaltenmatrix zusammengefasst,

$$
\frac{\partial \mathbf{u}^{*}}{\partial t}=\frac{\partial}{\partial t}\left[\begin{array}{l}
\mathbf{u}_{T} \\
\mathbf{u}_{D}
\end{array}\right]
$$

erhält man folgende Matrizengleichung für $\mathbf{F}_{M a N}$ :

$$
\mathbf{F}_{M a N}=C_{a} \mathbf{F}_{F K N}-C_{a} \rho\left[V \mathbf{T}_{N}-\left(V \mathbf{T}_{N} \mathbf{X}_{0 s}+I_{A \ell} \mathbf{T}_{N} \mathbf{E}_{T}\right)\right] \frac{\partial \mathbf{u}^{*}}{\partial t}
$$

Für das Moment der hydrodynamischen Massenkraft $\mathbf{M}_{M a N}$ folgt aus Gl. (B.11)

$$
\mathbf{M}_{M a N}=\int_{0}^{L} \mathbf{r} \times d \mathbf{F}_{M a N}=\int_{0}^{L} \mathbf{r} \times C_{a} \rho \mathrm{A}(\ell) \mathbf{T}_{N} \frac{\partial \mathbf{u}_{R}}{\partial t} d \ell
$$

Mit analogen Umformungen erhält man auch die folgende Matrizengleichung für $\mathbf{M}_{M a N}$

$$
\begin{aligned}
\mathbf{M}_{M a N}= & C_{a} \mathbf{M}_{F K N}- \\
& C_{a} \rho\left[\left(\begin{array}{r}
V \mathbf{X}_{0 s} \mathbf{T}_{N} \\
+I_{A \ell} \mathbf{E}_{T} \mathbf{T}_{N}
\end{array}\right):\left(\begin{array}{r}
-V \mathbf{X}_{0 s} \mathbf{T}_{N} \mathbf{X}_{0 s} \\
-I_{A \ell}\left(\mathbf{X}_{0 s} \mathbf{T}_{N} \mathbf{E}_{T}+\mathbf{E}_{T} \mathbf{T}_{N} \mathbf{X}_{0 s}\right) \\
-I_{A \ell 2} \mathbf{E}_{T} \mathbf{T}_{N} \mathbf{E}_{T}
\end{array}\right)\right] \frac{\partial \mathbf{u}^{*}}{\partial t}
\end{aligned}
$$


mit der Abkürzung:

$$
I_{A \ell 2}=\int_{0}^{L} A(\ell) \ell^{2} d \ell
$$

Abschließend lassen sich auch die Gleichungen (B.32) und (B.34) zu dem verallgemeinerten Kraftvektor $\mathbf{F}_{M a N}^{*}$ zusammenfassen:

$$
\mathbf{F}_{M a N}^{*}=C_{a} \mathbf{F}_{F K N}^{*}-C_{a} \rho\left[\begin{array}{rrr}
V \mathbf{T}_{N} & \vdots & -V \mathbf{T}_{N} \mathbf{X}_{0 s}-I_{A \ell} \mathbf{T}_{N} \mathbf{E}_{T} \\
\ldots \ldots \ldots \ldots \ldots \ldots \ldots \ldots \ldots \ldots \ldots \ldots \ldots \ldots \ldots & \vdots & \ldots \ldots \ldots \ldots \mathbf{X}_{0 s} \mathbf{T}_{N} \mathbf{X}_{0 s} \\
V \mathbf{X}_{0 s} \mathbf{T}_{N} & \vdots & -\mathbf{u}^{*} \\
+I_{A \ell} \mathbf{E}_{T} \mathbf{T}_{N} & \vdots & -I_{A \ell}\left(\mathbf{X}_{0 s} \mathbf{T}_{N} \mathbf{E}_{T}+\mathbf{E}_{T} \mathbf{T}_{N} \mathbf{X}_{0 s}\right) \\
& \vdots & -I_{A \ell 2} \mathbf{E}_{T} \mathbf{T}_{N} \mathbf{E}_{T}
\end{array}\right] \frac{\partial}{\partial t}
$$

Der zweite Term entspricht dem Anteil der hydrodynamischen Massenkraft, der durch die Beschleunigung des Körpers in ruhendem Wasser verursacht wird. Die Matrix mit dem Proportionalitätsfaktor $C_{a} \rho$ vor den Schwerpunktsbeschleunigungen $\partial \mathbf{u}^{*} / \partial t$ hat die Dimension $[\mathrm{kg}]$ und ist die hydrodynamische Masse eines quer zu seiner Achse beschleunigten Kreiszylinders. Die Matrix bewirkt die Transformation der beliebig gerichteten Schwerpunktsbeschleunigung auf die Normale der Zylinderachse. Für einen Kreiszylinder definiert man die Matrix der hydrodynamischen Masse A wie folgt:

$$
\mathbf{A}=C_{a} \rho\left[\begin{array}{rrr}
V \mathbf{T}_{N} & \vdots & -V \mathbf{T}_{N} \mathbf{X}_{0 s}-I_{A \ell} \mathbf{T}_{N} \mathbf{E}_{T} \\
\ldots \ldots \ldots \ldots \ldots \ldots \ldots \ldots \ldots & \vdots & -V \mathbf{X}_{0 s} \mathbf{T}_{N} \mathbf{X}_{0 s} \\
V \mathbf{X}_{0 s} \mathbf{T}_{N} & \vdots & -I_{A \ell}\left(\mathbf{X}_{0 s} \mathbf{T}_{N} \mathbf{E}_{T}+\mathbf{E}_{T} \mathbf{T}_{N} \mathbf{X}_{0 s}\right) \\
+I_{A \ell} \mathbf{E}_{T} \mathbf{T}_{N} & \vdots & -I_{A \ell 2} \mathbf{E}_{T} \mathbf{T}_{N} \mathbf{E}_{T}
\end{array}\right]
$$

\section{Zähigkeitsbedingte Widerstandskraft normal zur Zylinderachse}

Aus Gln. (B.9) und (B.11) folgt für die linearisierte Widerstandskraft normal zur Zylinderachse:

$$
\mathbf{F}_{D N}=\frac{\rho}{2} \frac{8}{3 \pi} \int_{0}^{L} C_{d} \mathbf{D}(\ell)\left[\mathbf{u}_{R N}\right]_{a} \mathbf{u}_{R N} d \ell
$$

Analog zu $\partial \mathbf{u}_{R N} / \partial t$ (Gl. B.25) erhält man die normal zur Zylinderachse gerichtete Relativgeschwindigkeit $\mathbf{u}_{R N}$ :

$$
\begin{aligned}
\mathbf{u}_{R N} & =\mathbf{T}_{N}\left(\mathbf{v}-\mathbf{u}_{T}-\mathbf{u}_{D} \times \mathbf{r}\right) \\
& =\mathbf{T}_{N}\left(\mathbf{v}-\mathbf{u}_{T}-\mathbf{X}_{0 s} \mathbf{u}_{D}+\ell \mathbf{E}_{T} \mathbf{u}_{D}\right)
\end{aligned}
$$

wobei das Kreuzprodukt durch die Matrizen $\mathbf{X}_{0 s}$ und $\mathbf{E}_{T}$ (Gl. B.18) ersetzt worden ist. 
Um die unbekannte Amplitude der Relativgeschwindigkeit $\left[\mathbf{u}_{R N}\right]_{a}$ als Funktion der Bewegungsvektoren $\mathbf{s}_{T}$ und $\mathbf{s}_{D}$ darstellen zu können, werden die Geschwindigkeitsvektoren $\mathbf{u}_{T}$ und $\mathbf{u}_{D}$ durch die Bewegungsvektoren $\mathbf{s}_{T}$ und $\mathbf{s}_{D}$ ersetzt. Mit dem harmonischen Ansatz für die Schwerpunktsbewegung s* (Siehe Gl. B.101) gilt die folgende Beziehung:

$$
\left(\begin{array}{l}
\mathbf{u}_{T} \\
\mathbf{u}_{D}
\end{array}\right)=\frac{\partial}{\partial t}\left(\begin{array}{c}
\mathbf{s}_{T} \\
\mathbf{s}_{D}
\end{array}\right)=-i \omega\left(\begin{array}{c}
\mathbf{s}_{T} \\
\mathbf{s}_{D}
\end{array}\right)
$$

Aus Gln. (B.40) und (B.39) ergibt sich für $\left[\mathbf{u}_{R N}\right]_{a}$ :

$$
\left[\mathbf{u}_{R N}\right]_{a}=\left|\mathbf{T}_{N}\left(\mathbf{v}+i \omega \mathbf{s}_{T}-i \omega \mathbf{X}_{0 s} \mathbf{s}_{D}-i \omega \ell \mathbf{E}_{T} \mathbf{s}_{D}\right)\right|
$$

Die beiden unbekannten Größen $\mathbf{s}_{T}$ und $\mathbf{s}_{D}$ werden später für die Ermittlung der Übertragungsfunktion auf die Wellenamplitude $\zeta_{a}$ normiert. Wird die Wellenamplitude $\zeta_{a}$ aus Gl. (B.41) herausgezogen, folgt:

$$
\left[\mathbf{u}_{R N}\right]_{a}=\zeta_{a} \cdot\left|\mathbf{T}_{N}\left(\widetilde{\mathbf{v}}+i \omega \widetilde{\mathbf{s}}_{T}-i \omega \mathbf{X}_{0 s} \widetilde{\mathbf{s}}_{D}-i \omega \ell \mathbf{E}_{T} \widetilde{\mathbf{s}}_{D}\right)\right|
$$

mit den Abkürzungen

$$
\widetilde{\mathbf{v}}=\frac{\mathbf{v}}{\zeta_{a}}, \quad \widetilde{\mathbf{s}}_{T}=\frac{\mathbf{s}_{T}}{\zeta_{a}} \quad \text { und } \quad \widetilde{\mathbf{s}}_{D}=\frac{\mathbf{s}_{D}}{\zeta_{a}}
$$

Bei Gl. (B.42) ist festzustellen, dass die Amplitude der Relativgeschwindigkeit $\left[\mathbf{u}_{R N}\right]_{a}$ von den unbekannten Bewegungsvektoren abhängt. Demzufolge ist die Widerstandskraft auch mit den Bewegungsvektoren $\mathbf{s}_{T}$ und $\mathbf{s}_{D}$ gekoppelt.

Werden Gln. (B.42) und (B.41) in die Gl. (B.38) eingesetzt, ergibt sich für $\mathbf{F}_{D N}$ :

$$
\mathbf{F}_{D N}=\frac{\rho}{2} \frac{8}{3 \pi} C_{d} \mathbf{T}_{N}\left(\begin{array}{c}
\int_{0}^{L} \mathbf{D}(\ell) \mathbf{v} d \ell-\mathbf{u}_{T} \int_{0}^{L} \mathbf{D}(\ell)\left[\mathbf{u}_{R N}\right]_{a} d \ell \\
+\mathbf{X}_{0 s} \mathbf{u}_{D} \int_{0}^{L} \mathbf{D}(\ell)\left[\mathbf{u}_{R N}\right]_{a} d \ell+\mathbf{E}_{T} \mathbf{u}_{D} \int_{0}^{L} \mathbf{D}(\ell) \ell\left[\mathbf{u}_{R N}\right]_{a} d \ell
\end{array}\right)
$$

Der Widerstandsbeiwert $C_{d}$ wird hier ebenfalls als konstant über $\ell$ angenommen. Die Geschwindigkeitsvektoren $\mathbf{u}_{T}$ und $\mathbf{u}_{D}$ sind von der Integrationsvariablen $\ell$ unabhängig.

Mit der Spaltenmatrix

$$
\mathbf{u}^{*}=\left(\begin{array}{l}
\mathbf{u}_{T} \\
\mathbf{u}_{D}
\end{array}\right)
$$

lässt sich Gl. (B.44) wieder zu einer Matrizengleichung zusammenfassen:

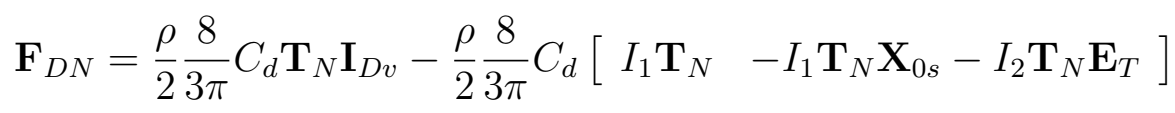

Dabei gelten folgende Abkürzungen:

$$
\mathbf{I}_{D v}=\int_{0}^{L} \mathbf{D} \mathbf{v} d \ell \quad I_{1}=\int_{0}^{L} \mathbf{D}\left[\mathbf{u}_{R N}\right]_{a} d \ell \quad I_{2}=\int_{0}^{L} \mathbf{D} \ell\left[\mathbf{u}_{R N}\right]_{a} d \ell
$$


Für das Moment der linearisierten Widerstandskraft erhält man durch analoge Umformungen folgende Matrizengleichung:

$$
\begin{aligned}
\mathbf{M}_{D N}= & \frac{\rho}{2} \frac{8}{3 \pi} C_{d}\left[\begin{array}{lll}
\mathbf{X}_{0 s} \mathbf{T}_{N} & \vdots & \mathbf{E}_{T} \mathbf{T}_{N}
\end{array}\right]\left[\begin{array}{c}
\mathbf{I}_{D v} \\
\mathbf{I}_{D v \ell}
\end{array}\right] \\
& -\frac{\rho}{2} \frac{8}{3 \pi} C_{d}\left[\begin{array}{rrr}
I_{1} \mathbf{X}_{0 s} \mathbf{T}_{N} & \vdots & -I_{1} \mathbf{X}_{0 s} \mathbf{T}_{N} \mathbf{X}_{0 s} \\
+I_{2} \mathbf{E}_{T} \mathbf{T}_{N} & \vdots & -I_{2}\left(\mathbf{X}_{0 s} \mathbf{T}_{N} \mathbf{E}_{T}+\mathbf{E}_{T} \mathbf{T}_{N} \mathbf{X}_{0 s}\right) \\
& \vdots & -I_{3} \mathbf{E}_{T} \mathbf{T}_{N} \mathbf{E}_{T}
\end{array}\right] \mathbf{u}^{*}
\end{aligned}
$$

mit den Abkürzungen

$$
\mathbf{I}_{3}=\int_{0}^{L} \mathbf{D} \ell^{2}\left[\mathbf{u}_{R N}\right]_{a} d \ell \quad \mathbf{I}_{D v \ell}=\int_{0}^{L} \mathbf{D} \ell \mathbf{v} d \ell
$$

Abschließend erhält man wieder den verallgemeinerten Kraftvektor aus Gln. (B.46) und (B.48):

$$
\begin{aligned}
\mathbf{F}_{D N}^{*}= & \frac{\rho}{2} \frac{8}{3 \pi} C_{d}\left[\begin{array}{cr}
\mathbf{T}_{N} & \mathbf{0} \\
\mathbf{X}_{0 s} \mathbf{T}_{N} & \mathbf{E}_{T} \mathbf{T}_{N}
\end{array}\right]\left[\begin{array}{c}
\mathbf{I}_{D v} \\
\mathbf{I}_{D v \ell}
\end{array}\right] \\
& -\frac{\rho}{2} \frac{8}{3 \pi} C_{d}\left[\begin{array}{rrr}
I_{1} \mathbf{T}_{N} & \vdots & -I_{1} \mathbf{T}_{N \mathbf{X}_{0 s}}-I_{2} \mathbf{T}_{N} \mathbf{E}_{T} \\
\ldots \ldots \ldots & \vdots & \ldots \ldots \ldots \ldots \ldots \ldots \ldots \\
I_{1} \mathbf{X}_{0 s} \mathbf{T}_{N} & \vdots & -I_{1} \mathbf{X}_{0 s} \mathbf{T}_{N} \mathbf{X}_{0 s} \\
+I_{2} \mathbf{E}_{T} \mathbf{T}_{N} & \vdots & -I_{2}\left(\mathbf{X}_{0 s} \mathbf{T}_{N} \mathbf{E}_{T}+\mathbf{E}_{T} \mathbf{T}_{N} \mathbf{X}_{0 s}\right) \\
\vdots & -I_{3} \mathbf{E}_{T} \mathbf{T}_{N} \mathbf{E}_{T}
\end{array}\right] \mathbf{u}^{*}
\end{aligned}
$$

Der zweite Anteil entspricht der zähigkeitsbedingten Dämpfung, die durch die Bewegung des Körpers in unbewegtem Wssser verursacht wird. Mit dem konstanten Faktor $4 \rho C_{d} / 3 \pi$ hat die Matrix vor $\mathbf{u}^{*}$ die Dimension $[N \cdot s / m]$. Sie hat die gleichen Matrizenkomponenten wie die Matrix der hydrodynamischen Masse A bis auf die Integrationskonstanten (vgl. Gl. B.37). Analog zu A lässt sich hierbei eine Dämpfungsmatrix B definieren:

$$
\mathbf{B}=\frac{\rho}{2} \frac{8}{3 \pi} C_{d}\left[\begin{array}{rrr}
-I_{1} \mathbf{T}_{N} & \vdots & -I_{1} \mathbf{T}_{N} \mathbf{X}_{0 s}-I_{2} \mathbf{T}_{N} \mathbf{E}_{T} \\
\ldots \ldots \ldots & \vdots & \ldots \ldots \ldots \ldots \ldots \ldots \cdots \\
I_{1} \mathbf{X}_{0 s} \mathbf{T}_{N} & \vdots & I_{1} \mathbf{X}_{0 s} \mathbf{T}_{N} \mathbf{X}_{0 s} \\
+I_{2} \mathbf{E}_{T} \mathbf{T}_{N} & \vdots & -I_{2}\left(\mathbf{X}_{0 s} \mathbf{T}_{N} \mathbf{E}_{T}+\mathbf{E}_{T} \mathbf{T}_{N} \mathbf{X}_{0 s}\right) \\
& \vdots & -I_{3} \mathbf{E}_{T} \mathbf{T}_{N} \mathbf{E}_{T}
\end{array}\right]
$$

Wie oben bei Gl. (B.42) gezeigt, ist hierbei zu beachten, dass die Dämpfungsmatrix sowohl von der Wellenamplitude als auch von den unbekannten Bewegungsvektoren $\mathbf{s}_{T}$ und $\mathbf{s}_{D}$ abhängig ist. Deshalb ist es erforderlich, die Dämpfungsmatrix sowie die Widerstandskraft iterativ zu ermitteln (siehe Abschnitt B.6). 


\section{B.3.2 Kraft in Richtung der Zylinderlängsachse}

Wie dem Zylindermantel können auch den benetzten Endflächen die Anteile der hydrodynamischen Kräfte zugeordnet werden. Da die Querschnittsfläche des Strukturelements veränderlich sein kann, müssen neben den Kräften auf die Endflächen die zusätzlichen Kräfte berücksichtigt werden, die auf die in Längsrichtung des Zylinders projizierten Mantelflächen wirken.

Für die Berechnung der hydrodynamischen Kräfte (in Längsrichtung) auf ein Strukturelement mit variablem Kreisquerschnitt wird folgendes Berechnungsmodell verwendet: Man zerlegt das Strukturelement in mehrere Kreisscheiben, um die Kraft auf die projizierte Oberfläche näherungsweise zu ermitteln (siehe Abb. B.4). Die Kraft auf die Endflächen wird dann getrennt berechnet. Es wird ebenfalls vorausgesetzt, dass die Beschleunigungen und Geschwindigkeiten von Wasserpartikeln nicht an der Körperoberfläche, sondern am Ort der Zylinderachse berechnet werden.

Im folgenden werden die Kraftanteile in Längsrichtung der Zylinderachse einzeln behandelt. Die auftretenden Terme werden dabei ebenfalls in Form von Matrizen zusammengefasst. Für schlanke Kreiszylinder ist der Anteil der zähigkeitsbedingten Widerstandskraft in Längsrichtung bedeutend kleiner als die Trägheitskraft und wird hier vernachlässigt.

\section{Froude-Krylov-Kraft in Längsrichtung}

Der Betrag der Druckkraft auf die Endfläche errechnet sich näherungsweise als das Produkt der Endfläche $A_{e}$ und dem dynamischen Druck $p_{d y n}$ im Schwerpunkt der Fläche. Mit dem in die Flüssigkeit zeigenden Normalenvektor $\mathbf{n}_{e}=-\mathbf{e}_{T}$ der Fläche $A_{e}$ ist die Kraft des dynamischen Druckes $\mathbf{F}_{p e}$ auf die benetzte Endfläche $A_{e}$ :

$$
\mathbf{F}_{p e}=-\mathbf{n}_{e} A_{e} p_{d y n}
$$

Mit dem Ortvektor $\mathrm{r}_{e}$ vom Schwerpunkt $\mathbf{S}$ zum Flächenmittelpunkt folgt das Moment der Kraft auf die Endfläche:

$$
\mathbf{M}_{p e}=-\mathbf{r}_{e} \times \mathbf{n}_{e} A_{e} p_{d y n}
$$

Zur Ermittlung der Druckkraft auf die projizierte Oberfläche betrachtet man eine Kreisscheibe des Zylinders (Abb. B.4). Der Betrag der projizierten Oberfläche eines Scheibenelementes in Längsrichtung der Zylinderachse lässt sich näherungsweise als Differenz der beiden benachbarten Querschnittsflächen berechnen. Die Druckkraft auf eine Kreisscheibe ergibt sich mit dem Einheitsvektor $\mathbf{e}_{T}$ :

$$
\delta \mathbf{F}_{p m}^{j}=\left(A_{j+1}-A_{j}\right) p_{d y n j}(z) \mathbf{e}_{T}
$$

Für die gesamte Kraft auf die Oberfläche in Längsrichtung gilt also:

$$
\mathbf{F}_{p m}=\sum_{j}\left(A_{j+1}-A_{j}\right) p_{d y n j} \mathbf{e}_{T}
$$



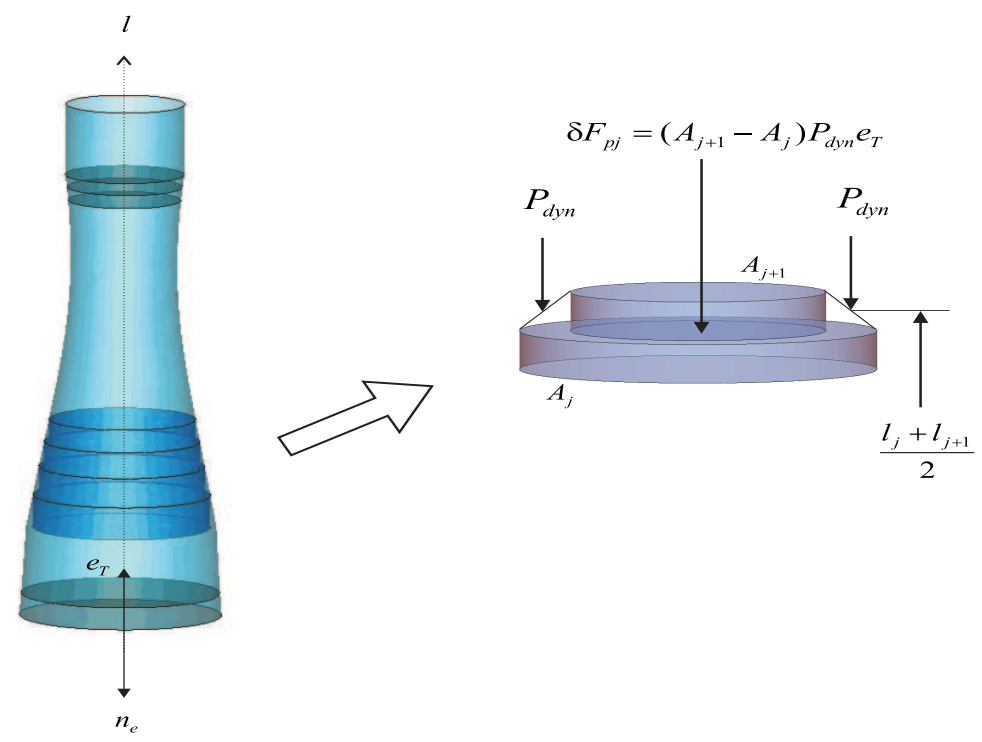

Abbildung B.4: Berechnungsmodell zur Ermittlung der Druckkraft auf die in Längsrichtung eines Strukturelementes projizierte Fläche

wobei der Zähler $j$ die j-te Endfläche der Kreisscheibe bezeichnet. Für das Moment der Druckkraft auf die Oberfläche in Längsrichtung gilt dann analog zu Gl. (B.53):

$$
\mathbf{M}_{p m}=\sum_{j} \mathbf{r}_{j} \times\left(A_{j+1}-A_{j}\right) p_{d y n j} \mathbf{e}_{T}
$$

Zusammengefasst erhält man als verallgemeinerten Kraftvektor $F_{p}^{*}$ :

$$
\mathbf{F}_{p}^{*}=\left(\begin{array}{c}
-\mathbf{n}_{e} A_{e} p_{d y n}+\sum_{j}\left(A_{j+1}-A_{j}\right) p_{d y n j} \mathbf{e}_{T} \\
-\mathbf{r}_{e} \times \mathbf{n}_{e} A_{e} p_{d y n}+\sum_{j} r_{j} \times\left(A_{j+1}-A_{j}\right) p_{d y n j} \mathbf{e}_{T}
\end{array}\right)
$$

\section{Hydrodynamische Massenkraft in Längsrichtung}

Für Strukturen mit konstanten Querschnittsflächen kann die hydrodynamische Masse in Längsrichtung nach Clauss et al. (1992) in folgender Weise berechnet werden:

$$
\begin{aligned}
\text { für einen Caisson: } & m_{h}=\frac{8}{3} \rho C_{a}\left(\alpha_{c}\right) R_{c}^{3} \\
\text { für eine Säule: } & m_{h}=\frac{4}{3} \rho C_{a}\left(\alpha_{s}\right) R_{s}^{3}
\end{aligned}
$$

Der hydrodynamische Massenbeiwert $C_{a}$ wird für den Durchmesser-Längen-Parameter $\alpha_{c}$ bei Caissons (vollgetaucht) bzw. $\alpha_{s}$ bei Säulen (durchstoßen die Wasseroberfläche) aus Abb. B.5 bestimmt.

Für die Berechnung der hydrodynamischen Massenkraft in Längsrichtung des Strukturelements benötigt man analog zu $\partial \mathbf{u}_{R N} / \partial t$ die Projektion $\partial \mathbf{u}_{R T} / \partial t$ von $\partial \mathbf{u}_{R} / \partial t$ in 


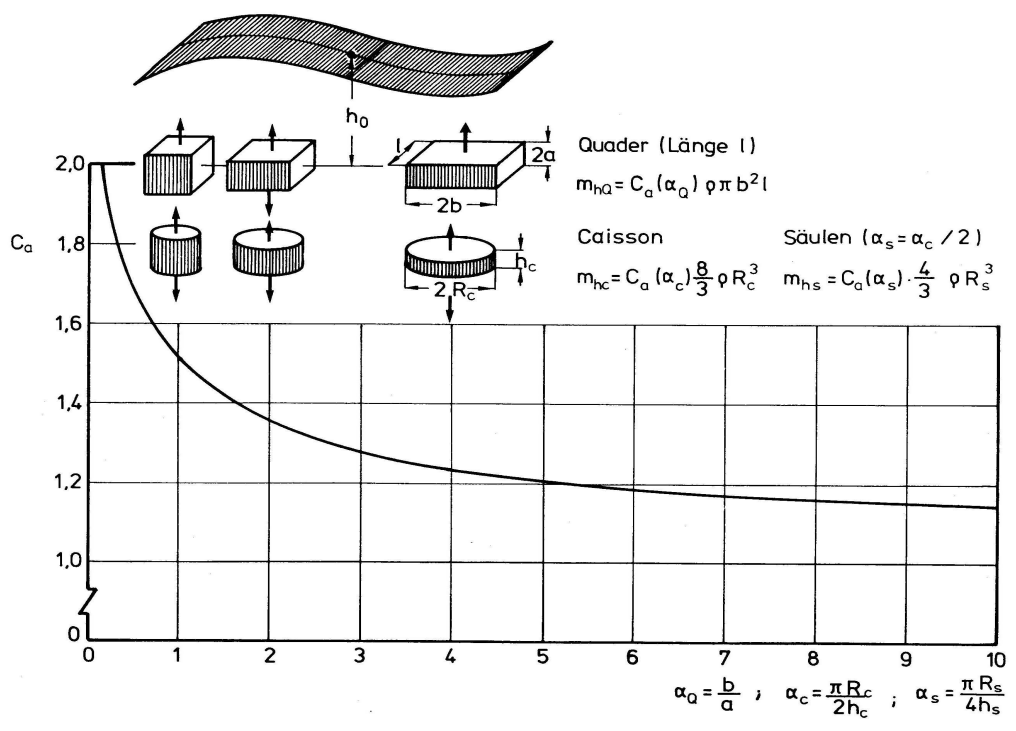

Abbildung B.5: Beiwerte der hydrodynamischen Masse für harmonisch in Axialrichtung schwingende Körper (Clauss et al., 1992)

Richtung der Zylinderachse. Der Vektor der Relativbeschleunigung in Richtung der Zylinderachse $\partial \mathbf{u}_{R T} / \partial t$ ergibt sich wie folgt

$$
\frac{\partial \mathbf{u}_{R T}}{\partial t}=\left(\frac{\partial \mathbf{u}_{R}}{\partial t} \cdot \mathbf{e}_{T}\right) \mathbf{e}_{T}=\left(\mathbf{e}_{T}^{T} \frac{\partial \mathbf{u}_{R}}{\partial t}\right) \mathbf{e}_{T}=\left(\mathbf{e}_{T} \cdot \mathbf{e}_{T}^{T}\right) \frac{\partial \mathbf{u}_{R}}{\partial t}=\mathbf{N}_{T} \frac{\partial \mathbf{u}_{R}}{\partial t} .
$$

Die Transformationsmatrix $\mathbf{N}_{T}$ berechnet sich aus dem dyadischen Produkt $\mathbf{e}_{T} \cdot \mathbf{e}_{T}^{T}$ :

$$
\mathbf{N}_{T}=\left[\begin{array}{ccc}
e_{T x}^{2} & e_{T x} e_{T y} & e_{T x} e_{T z} \\
e_{T x} e_{T y} & e_{T y}^{2} & e_{T y} e_{T z} \\
e_{T x} e_{T z} & e_{T y} e_{T z} & e_{T z}^{2}
\end{array}\right]
$$

Bei der Berechnung der hydrodynamischen Massenkraft in Längsrichtung wird vorausgesetzt, dass der Angriffspunkt der Kraft in den Volumenschwerpunkt des Strukturelementes gelegt werden kann. Für diesen Punkt wird auch die Relativbeschleunigung $\partial \mathbf{u}_{R T} / \partial t$ bestimmt. Bei schlanken Zylindern ist die hydrodynamische Masse klein und somit der Einfluss der hydrodynamischen Kraft in Längsrichtung insgesamt klein. Die Auswahl des Volumenschwerpunktes als Angriffspunkt spielt deshalb keine wesentliche Rolle. Aus Gl. (B.58) und (B.59) ergibt sich für die hydrodynamische Massenkraft in Längsrichtung $\mathbf{F}_{M a T}$ :

$$
\mathbf{F}_{M a T}=m_{h} \mathbf{N}_{T} \frac{\partial \mathbf{u}_{R}}{\partial t}=m_{h} \mathbf{N}_{T}\left(\frac{\partial \mathbf{v}}{\partial t}-\frac{\partial \mathbf{u}_{T}}{\partial t}-\frac{\partial \mathbf{u}_{D}}{\partial t} \times \mathbf{r}_{m}\right)
$$

Dabei bezeichnet $\mathbf{r}_{m}$ den Ortsvektor vom Schwerpunkt $S$ zu dem Volumenschwerpunkt des Strukturelementes mit $\mathbf{r}_{m}=\left(r_{m x}, r_{m y}, r_{m z}\right)^{T}$. Wird das Kreuzprodukt $\partial \mathbf{u}_{D} / \partial t \times \mathbf{r}_{m}$ 
ebenfalls gegen eine Matrizenmultiplikation ausgetauscht

$$
\frac{\partial \mathbf{u}_{D}}{\partial t} \times \mathbf{r}_{m}=\left[\begin{array}{ccc}
0 & r_{m z} & -r_{m y} \\
-r_{m z} & 0 & r_{m x} \\
r_{m y} & -r_{m x} & 0
\end{array}\right] \frac{\partial \mathbf{u}_{D}}{\partial t}=\mathbf{R}_{m} \frac{\partial \mathbf{u}_{D}}{\partial t}
$$

folgt aus Gl. (B.61):

$$
\mathbf{F}_{M a T}=m_{h} \mathbf{N}_{T} \frac{\partial \mathbf{v}}{\partial t}-m_{h}\left[\begin{array}{ll}
\mathbf{N}_{T} & \mathbf{N}_{T} \mathbf{R}_{m}
\end{array}\right] \frac{\partial \mathbf{u}^{*}}{\partial t}
$$

Das entsprechende Moment erhält man mit analogen Umformungen

$$
\begin{aligned}
\mathbf{M}_{M a T} & =m_{h} \cdot\left(r_{m} \times \frac{\partial \mathbf{u}_{R T}}{\partial t}\right) \\
& =m_{h}\left[-\mathbf{R}_{m} \mathbf{N}_{T}\right] \frac{\partial \mathbf{v}}{\partial t}-m_{h}\left[-\mathbf{R}_{m} \mathbf{N}_{T} \quad-\mathbf{R}_{m} \mathbf{N}_{T} \mathbf{R}_{m}\right] \frac{\partial \mathbf{u}^{*}}{\partial t}
\end{aligned}
$$

Fasst man abschließend Kraft und Moment zu einer Spaltenmatrix zusammen, so ergibt sich:

$$
\mathbf{F}_{M a T}^{*}=m_{h}\left[\begin{array}{c}
\mathbf{N}_{T} \\
-\mathbf{R}_{m} \mathbf{N}_{T}
\end{array}\right] \frac{\partial \mathbf{v}}{\partial t}-m_{h}\left[\begin{array}{cc}
\mathbf{N}_{T} & \mathbf{N}_{T} \mathbf{R}_{m} \\
-\mathbf{R}_{m} \mathbf{N}_{T} & -\mathbf{R}_{m} \mathbf{N}_{T} \mathbf{R}_{m}
\end{array}\right] \frac{\partial \mathbf{u}^{*}}{\partial t}
$$

Für die Berechnung der hydrodynamischen Masse wurde eine Funktion programmiert. Sie enthält eine interpolierte B-Spline-Kurve als Näherung für die in Abb. B.5 gezeigte Kurve des Beiwertes $C_{a}$. Der zum aktuellen Parameter $\alpha$ gehörende $C_{a}$-Wert wird durch diese Funktion bestimmt.

Für Strukturen mit veränderlichem Durchmesser kann die hydrodynamische Masse nicht direkt durch Abb. B.5 berechnet werden. Da der Anteil der hydrodynamischen Masse infolge der veränderlichen Querschnittsflächen zusätzlich berücksichtigt werden muss, wird im folgenden die hydrodynamischen Massenkraft in Längsrichtung wie bei Froude-Krylov-Kraft auf Endflächen und auf die Mantelflächen getrennt ermittelt.

Nach Clauss et al. (1992) kann die hydrodynamische Masse für eine in Axialrichtung schwingende Kreisscheibe wie folgt berechnet werden:

$$
m_{h}=\frac{8}{3} \rho R^{3}
$$

Bei Endflächen des Strukturelementes ist nur eine Querschnittsfläche benetzt, so dass für die hydrodynamische Masse der halbe Wert der für eine Kreisscheibe gültigen Größe anzusetzen ist. Aus (B.66) folgt also:

$$
m_{h e}=\frac{4}{3} \rho R_{e}^{3}
$$

Für die hydrodynamische Massenkraft auf die Endfläche $A_{e}$ erhält man analog zu Gl. (B.61):

$$
\mathbf{F}_{M a T e}=m_{h e} \mathbf{N}_{T} \frac{\partial \mathbf{u}_{R}}{\partial t}=m_{h e} \mathbf{N}_{T}\left(\frac{\partial \mathbf{v}}{\partial t}-\frac{\partial \mathbf{u}_{T}}{\partial t}-\frac{\partial \mathbf{u}_{D}}{\partial t} \times \mathbf{r}_{0}\right)
$$


Dabei wird der Angriffspunkt der Kraft in den Mittelpunkt der Endfläche gelegt. Ana$\log$ zu Gl. (B.65) erhält man durch gezielte Umformungen folgende Matrizengleichung für die hydrodynamische Massenkraft und das entsprechende Moment auf die Endfläche $A_{e}$ :

$$
\mathbf{F}_{M a T e}^{*}=m_{h e}\left[\begin{array}{c}
\mathbf{N}_{T} \\
\mathbf{X}_{0 s} \mathbf{N}_{T}
\end{array}\right] \frac{\partial \mathbf{v}}{\partial t}-m_{h e}\left[\begin{array}{cc}
\mathbf{N}_{T} & \mathbf{N}_{T} \mathbf{X}_{0 s} \\
\mathbf{X}_{0 s} \mathbf{N}_{T} & -\mathbf{X}_{0 s} \mathbf{N}_{T} \mathbf{X}_{0 s}
\end{array}\right] \frac{\partial \mathbf{u}^{*}}{\partial t}
$$

Die Gl. (B.69) gilt für ein Strukturelement, das nur eine benetzte Endfläche enthält, z.B. eine Säule eines Halbtauchers. Für ein vollgetauchtes Strukturelement muss deshalb die Kraft auf die zweite Endfläche zu Gl. (B.69) addiert werden.

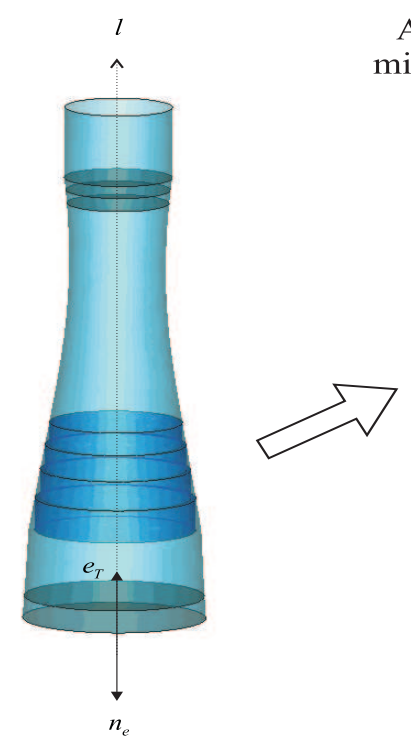

Ansatz für die hydrodynamische Masse
mit zwei benachbarten Scheibenelementen

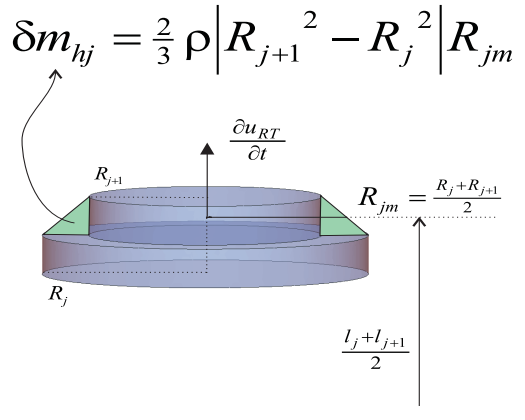

Abbildung B.6: Näherungsansatz für die hydrodynamische Masse mit zwei benachbarten Scheibenelementen

Um den Anteil der hydrodynamische Massenkraft auf die Mantelfläche (in Längsrichtung) näherungsweise zu ermitteln, wird ebenfalls ein Berechnungsmodell verwendet, in dem das veränderliche Strukturelement in mehrere Scheibenelemente aufgeteilt wird (siehe Abb. B.6). Es wird dabei angenommen, dass sich der zusätzliche Anteil der hydrodynamischen Masse für veränderliche Querschnittsflächen durch Differenz der hydrodynamischen Massen zwischen zwei benachbarten Scheibenelementen errechnen lässt. Der Näherungsansatz unter Verwendung von Gl. (B.67) lautet folglich:

$$
\delta m_{h j}=\frac{1}{2} \frac{4}{3} \rho\left|R_{j+1}^{2}-R_{j}^{2}\right| R_{j m} \quad \text { mit } \quad R_{j m}=\frac{R_{j+1}+R_{j}}{2}
$$

Wie in Abb. B.6 dargestellt, entspricht die Differenz der Volumina zwischen beiden Scheibenelementen geometrisch dem doppelten Wert der eigentlichen Volumendifferenz zwischen der ursprünglichen Struktur und dem Scheibenmodell. Aus diesem Grund 
wurde der Faktor 1/2 eingeführt, so dass bei der Approximation die Änderung der hydrodynamischen Masse entsprechend berücksichtigt wird. Da die hydrodynamische Masse nicht negativ werden kann, wird die Änderung der Querschnittsfläche als Betrag erfasst. Daraus folgt für die hydrodynamische Massenkraft auf ein Scheibenelement:

$$
\begin{aligned}
\delta \mathbf{F}_{M a T m} & =\delta m_{h j} \frac{\partial \mathbf{u}_{R T}}{\partial t} \\
& =\frac{2}{3} \rho\left|R_{j+1}^{2}-R_{j}^{2}\right| R_{j m} \mathbf{N}_{T} \frac{\partial \mathbf{u}_{R}}{\partial t}
\end{aligned}
$$

Der Angriffspunkt wird ebenfalls in den Schwerpunkt des Scheibenelementes gelegt. Die Beschleunigung $\partial \mathbf{u}_{R T} / \partial t$ wird bezogen auf diesen Punkt berechnet. Für die Kraft auf die gesamte Mantelfläche ergibt sich schließlich:

$$
\begin{aligned}
\mathbf{F}_{M a T m} & =\sum_{j} \frac{2}{3} \rho\left|R_{j+1}^{2}-R_{j}^{2}\right| R_{j m} \mathbf{N}_{T} \frac{\partial \mathbf{u}_{R}}{\partial t} \\
& =\sum_{j} \frac{2}{3} \rho\left|R_{j+1}^{2}-R_{j}^{2}\right| R_{j m} \mathbf{N}_{T}\left(\frac{\partial \mathbf{v}}{\partial t}-\frac{\partial \mathbf{u}_{T}}{\partial t}-\frac{\partial \mathbf{u}_{D}}{\partial t} \times \mathbf{r}_{j m}\right)
\end{aligned}
$$

Um diese Gleichung in eine übersichtliche Matrizengleichung zu transformieren, ersetzt man das Kreuzprodukt durch äquivalente Matrizen $\mathbf{X}_{0 s}$ und $\mathbf{E}_{T}$ (Gl. B.18)

$$
\begin{aligned}
\mathbf{F}_{\text {MaTm }}= & \frac{2}{3} \rho \sum_{j}\left|R_{j+1}^{2}-R_{j}^{2}\right| R_{j m} \mathbf{N}_{T} \frac{\partial \mathbf{v}}{\partial t} \\
& -\frac{2}{3} \rho\left[\begin{array}{rr}
\sum_{j}\left|R_{j+1}^{2}-R_{j}^{2}\right| R_{j m} \mathbf{N}_{T} \vdots & -\left(\sum_{j}\left|R_{j+1}^{2}-R_{j}^{2}\right| R_{j m} \mathbf{N}_{T} \mathbf{X}_{0 s}\right. \\
\vdots & \left.\left.+\sum_{j}\left|R_{j+1}^{2}-R_{j}^{2}\right| R_{j m} \ell_{j m} \mathbf{N}_{T} \mathbf{E}_{T}\right)\right] \frac{\partial \mathbf{u}^{*}}{\partial t}
\end{array}\right.
\end{aligned}
$$

Für das Moment der hydrodynamischen Massenkraft $\mathbf{M}_{M a T m}$ gilt nach Gl. (B.71):

$$
\mathbf{M}_{M a T m}=\sum_{j}\left(\mathbf{r}_{j m} \times \delta \mathbf{F}_{M a T}\right)
$$

Mit den Abkürzungen

$$
\begin{aligned}
\mathbf{S}_{v} & =\sum_{j}\left|R_{j+1}^{2}-R_{j}^{2}\right| R_{j m} \frac{\partial \mathbf{v}}{\partial t} \\
\mathbf{S}_{v \ell} & =\sum_{j}\left|R_{j+1}^{2}-R_{j}^{2}\right| R_{j m} \ell_{j m} \frac{\partial \mathbf{v}}{\partial t} \\
S_{1} & =\sum_{j}\left|R_{j+1}^{2}-R_{j}^{2}\right| R_{j m} \\
S_{2} & =\sum_{j}\left|R_{j+1}^{2}-R_{j}^{2}\right| R_{j m} \ell_{j m} \\
S_{3} & =\sum_{j}\left|R_{j+1}^{2}-R_{j}^{2}\right| R_{j m} \ell_{j m}^{2}
\end{aligned}
$$


ergibt sich das Moment $\mathbf{M}_{M a T m}$ in Form von Matrizen

$$
\begin{aligned}
\mathbf{M}_{M a T m}= & \frac{2}{3} \rho\left[\begin{array}{ll}
\mathbf{X}_{0 s} \mathbf{N}_{T} & \left.\mathbf{E}_{T} \mathbf{N}_{T}\right]
\end{array}\right]\left[\begin{array}{c}
\mathbf{S}_{v} \\
\mathbf{S}_{v \ell}
\end{array}\right] \\
& -\frac{2}{3} \rho\left[\begin{array}{ccc}
S_{1} \mathbf{X}_{0 s} \mathbf{N}_{T} & \vdots & -\left(S_{1} \mathbf{X}_{0 s} \mathbf{N}_{T} \mathbf{X}_{0 s}+S_{2} \mathbf{X}_{0 s} \mathbf{N}_{T} \mathbf{E}_{T}\right. \\
+S_{2} \mathbf{E}_{T} \mathbf{N}_{T} & \vdots & \left.+S_{2} \mathbf{E}_{T} \mathbf{N}_{T} \mathbf{X}_{0 s}+S_{3} \mathbf{E}_{T} \mathbf{N}_{T} \mathbf{E}_{T}\right)
\end{array}\right] \frac{\partial \mathbf{u}^{*}}{\partial t}
\end{aligned}
$$

Abschließend lassen sich die Gln. (B.73) und (B.76) wieder zu dem verallgemeinerten Kraftvektor $\mathbf{F}_{\text {Matm }}^{*}$ in einer Spaltenmatrix zusammenfassen:

$$
\begin{aligned}
& \mathbf{F}_{M a T m}^{*}=\frac{2}{3} \rho\left[\begin{array}{cc}
\mathbf{N}_{T} & \mathbf{0} \\
\mathbf{X}_{0 s} \mathbf{N}_{T} & \mathbf{E}_{T} \mathbf{N}_{T}
\end{array}\right]\left[\begin{array}{c}
\mathbf{S}_{v} \\
\mathbf{S}_{v \ell}
\end{array}\right] \\
& -\frac{2}{3} \rho\left[\begin{array}{lrr}
S_{1} \mathbf{N}_{T} & \vdots & -\left(S_{1} \mathbf{N}_{T} \mathbf{X}_{0 s}+S_{2} \mathbf{N}_{T} \mathbf{E}_{T}\right) \\
\cdots \ldots \ldots \ldots & \ldots \ldots \ldots \ldots \ldots \ldots \ldots \ldots \\
\left(S_{1} \mathbf{X}_{0 s} \mathbf{N}_{T} \vdots\right. & -\left(S_{1} \mathbf{X}_{0 s} \mathbf{N}_{T} \mathbf{X}_{0 s}+S_{2} \mathbf{X}_{0 s} \mathbf{N}_{T} \mathbf{E}_{T}\right. \\
\left.+S_{2} \mathbf{E}_{T} \mathbf{N}_{T}\right) \vdots & \left.+S_{2} \mathbf{E}_{T} \mathbf{N}_{T} \mathbf{X}_{0 s}+S_{3} \mathbf{E}_{T} \mathbf{N}_{T} \mathbf{E}_{T}\right)
\end{array}\right] \frac{\partial \mathbf{u}^{*}}{\partial t}
\end{aligned}
$$

Die gesamte Kraft (bzw. das Moment) auf einen Körper (z.B. eine Säule) erhält man aus Gln. (B.69) und (B.77)

$$
\mathbf{F}_{M a T}^{*}=\mathbf{F}_{M a T e}^{*}+\mathbf{F}_{M a T m}^{*} .
$$

Analog zu Gl. B.37 kann hierbei die Matrix der hydrodynamischen Masse in Längsrichtung $\mathbf{A}_{a x}$ definiert werden

$$
\begin{aligned}
& \mathbf{A}_{a x}= m_{h e}\left[\begin{array}{cc}
\mathbf{N}_{T} & \mathbf{N}_{T} \mathbf{X}_{0 s} \\
\mathbf{X}_{0 s} \mathbf{N}_{T} & -\mathbf{X}_{0 s} \mathbf{N}_{T} \mathbf{X}_{0 s}
\end{array}\right] \\
&+\frac{2}{3} \rho\left[\begin{array}{rrr}
S_{1} \mathbf{N}_{T} & \vdots & -\left(S_{1} \mathbf{N}_{T} \mathbf{X}_{0 s}+S_{2} \mathbf{N}_{T} \mathbf{E}_{T}\right) \\
\ldots \ldots \ldots \ldots \ldots \ldots \ldots \ldots & \ldots \ldots \ldots \ldots \ldots \ldots \ldots \\
\left(S_{1} \mathbf{X}_{0 s} \mathbf{N}_{T}\right. & \vdots & -\left(S_{1} \mathbf{X}_{0 s} \mathbf{N}_{T} \mathbf{X}_{0 s}+S_{2} \mathbf{X}_{0 s} \mathbf{N}_{T} \mathbf{E}_{T}\right. \\
\left.+S_{2} \mathbf{E}_{T} \mathbf{N}_{T}\right) & \left.+S_{2} \mathbf{E}_{T} \mathbf{N}_{T} \mathbf{X}_{0 s}+S_{3} \mathbf{E}_{T} \mathbf{N}_{T} \mathbf{E}_{T}\right)
\end{array}\right] .
\end{aligned}
$$




\section{B.3.3 Hydrostatische Rückstellkraft}

Die Rückstellkraft bzw. ihr Moment resultiert aus einer Änderung der hydrostatischen Druckverteilung am Körper. Diese verändert sich jedoch nur bei Tauchen, Rollen und Stampfen, d.h. bei Bewegungen, die Anteile in vertikaler Richtung besitzen. Bei Strukturelementen, die die Wasseroberfläche durchstoßen, führt die Druckänderung zu einer resultierenden Druckkraft $\mathbf{F}_{c}$ in z-Richtung, deren Betrag der Gewichtskraft des zusätzlich, bzw. weniger verdrängten Wassers entspricht. Da die Rückstellkraft $\mathbf{F}_{c}$ der Bewegung immer entgegengerichtet ist (siehe Abb. B.7), gilt

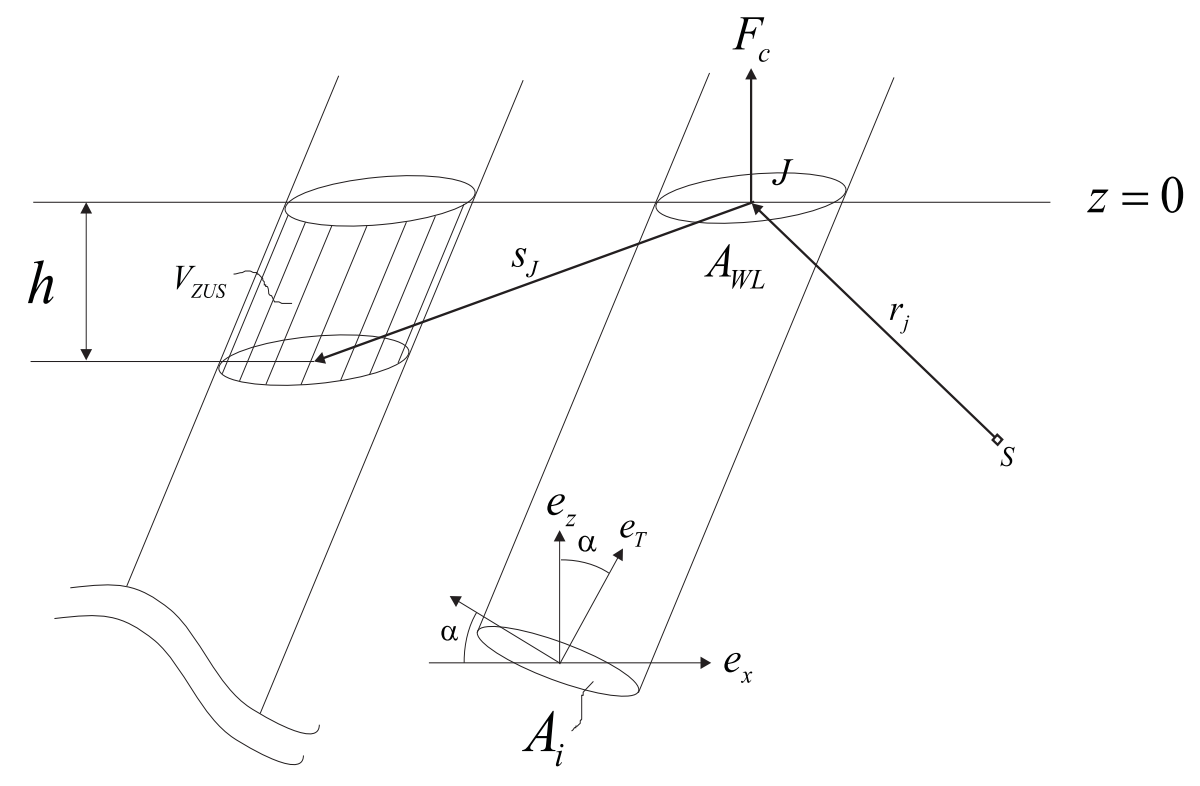

Abbildung B.7: Verdrängungszunahme bei kleinen Bewegungen

$$
\mathbf{F}_{c}=-\rho g V_{z u s} \mathbf{e}_{z}
$$

wobei die Verdrängungszunahme aus der Geometrie leicht zu bestimmen ist:

$$
V_{z u s}=h A_{W L}
$$

Es wird hierbei angenommen, dass bei kleinen Bewegungen (lineare Theorie) die Wasserlinienfläche konstant bleibt. Das bedeutet, dass sich die Querschnittsfläche in der Nähe der Wasseroberfläche nicht drastisch verändern darf. Die Höhe $h$ des Prismas gleicht der Länge der Projektion des Bewegungsvektors $\mathbf{s}_{j}$ auf den Einheitsvektor $\mathbf{e}_{z}$ :

$$
h=\mathbf{e}_{z}^{T} \mathbf{s}_{j}
$$

Bei konstantem Querschnitt ist die Wasserlinienfläche gleich $A_{i} / \cos \alpha$. Mit der Beziehung:

$$
\cos \alpha=\frac{\mathbf{e}_{T}^{T} \mathbf{e}_{z}}{\left|\mathbf{e}_{z}\right|\left|\mathbf{e}_{z}\right|}=\mathbf{e}_{T}^{T} \mathbf{e}_{z}
$$


ergibt sich für $\mathbf{F}_{c}$ :

$$
\begin{aligned}
\mathbf{F}_{c} & =-\rho g A_{i} / \cos \alpha\left(\mathbf{e}_{T}^{T} \mathbf{s}_{j}\right) \mathbf{e}_{z}=-\rho g A_{i} / \cos \alpha \mathbf{e}_{z}\left(\mathbf{e}_{T}^{T} \mathbf{s}_{j}\right) \\
& \left.=-\rho g A_{i} / \cos \alpha\left(\mathbf{e}_{z} \mathbf{e}_{T}^{T}\right) \mathbf{s}_{j}\right)
\end{aligned}
$$

Das dyadische Produkt $\mathbf{e}_{z} \mathbf{e}_{T}^{T}$ ergibt die schwach besetzte Matrix $\mathbf{E}_{z}$ :

$$
\mathbf{E}_{z}=\left[\begin{array}{ccc}
0 & 0 & 0 \\
0 & 0 & 0 \\
0 & 0 & 1
\end{array}\right]
$$

Die Bewegung des Wasserlinienschwerpunktes $j$ lässt sich bei kleinen Bewegungen aus der Translations- und Drehbewegung des Schwerpunktes ableiten:

$$
\mathbf{s}_{j}=\mathbf{s}_{T}+\mathbf{s}_{D} \times \mathbf{r}_{j}
$$

Damit folgt für die Rückstellkraft $\mathbf{F}_{c}$ :

$$
\mathbf{F}_{c}=-\rho g\left(A_{i} / \cos \alpha\right) \mathbf{E}_{z}\left(\mathbf{s}_{T}+\mathbf{s}_{D} \times \mathbf{r}_{j}\right)
$$

Um die unbekannten Vektoren $\mathbf{s}_{T}$ und $\mathbf{s}_{D}$ aus dem Ausdruck zu eliminieren, wird das Kreuzprodukt durch das äquivalente Matrizenprodukt ersetzt und man erhält:

$$
\begin{aligned}
\mathbf{F}_{c} & =-\rho g\left(A_{i} / \cos \alpha\right) \mathbf{E}_{z}\left(\mathbf{s}_{T}+\mathbf{R}_{j} \mathbf{s}_{D}\right) \\
& =-\rho g\left(A_{i} / \cos \alpha\right)\left[\begin{array}{ll}
\mathbf{E}_{z} & \mathbf{E}_{z} \mathbf{R}_{j}
\end{array}\right]\left[\begin{array}{l}
\mathbf{s}_{T} \\
\mathbf{s}_{D}
\end{array}\right] \\
& \text { mit } \quad \mathbf{R}_{j}=\left[\begin{array}{ccc}
0 & r_{j z} & -r_{j y} \\
-r_{j z} & 0 & r_{j x} \\
r_{j y} & -r_{j x} & 0
\end{array}\right]
\end{aligned}
$$

Die Kraft $\mathbf{F}_{c}$ bewirkt ein rückstellendes Moment $\mathbf{M}_{c}$ um den Schwerpunkt der Struktur. Als Angriffspunkt der Rückstellkraft wird dabei der Schwerpunkt der Wasserlinienfläche angenommen. Anteile aus dem Kippen des Zylinders um den Punkt $j$ selbst werden damit vernachlässigt. Dies entspricht der Vernachlässigung des Eigenflächenträgheitsmomentes der Wasserlinie, wenn rückstellende Momente über die metazentrische Höhe bestimmt werden. Bei Halbtauchern überwiegen jedoch aufgrund der großen Säulenabstände die Anteile nach Steiner am Wasserlinienträgheitsmoment. Für das Moment gilt folglich:

$$
\mathbf{M}_{c}=\mathbf{r}_{j} \times \mathbf{F}_{c}=-\mathbf{R}_{j} \mathbf{F}_{c}
$$

oder in Matrizenschreibweise :

$$
\mathbf{M}_{c}=-\rho g\left(A_{i} / \cos \alpha\right)\left[\begin{array}{ll}
-\mathbf{R}_{j} \mathbf{E}_{z} & -\mathbf{R}_{j} \mathbf{E}_{z} \mathbf{R}_{j}
\end{array}\right]\left[\begin{array}{c}
\mathbf{s}_{T} \\
\mathbf{s}_{D}
\end{array}\right]
$$


Abschließend lassen sich die Gleichungen (B.88) und (B.91) zu dem verallgemeinerten Kraftvektor $\mathbf{F}_{c}^{*}$ in einer Spaltenmatrix zusammenfassen:

$$
\mathbf{F}_{c}^{*}=-\rho g\left(A_{i} / \cos \alpha\right)\left[\begin{array}{cc}
\mathbf{E}_{z} & \mathbf{E}_{z} \mathbf{R}_{j} \\
-\mathbf{R}_{j} \mathbf{E}_{z} & -\mathbf{R}_{j} \mathbf{E}_{z} \mathbf{R}_{j}
\end{array}\right] \mathbf{s}^{*}
$$

Der Ausdruck $\rho g\left(A_{i} / \cos \alpha\right)$ hat die Dimension $[N / m]$ (wie eine Federkonstante). Das folgende Matrizenprodukt erzeugt aus der Schwerpunktsbewegung die tatsächliche Verschiebung der Wasserlinienfläche. Zusammen ergeben die Größen die lineare Rückstellkraft(bzw. -moment) eines Kreiszylinders, der die Wasseroberfläche durchstößst. Man definiert eine Matrix der Rückstellkoeffizienten $\mathbf{C}_{i}$ für den Kreiszylinder $i$ :

$$
\mathbf{C}_{i}=\rho g\left(A_{i} / \cos \alpha\right)\left[\begin{array}{cc}
\mathbf{E}_{z} & \mathbf{E}_{z} \mathbf{R}_{j} \\
-\mathbf{R}_{j} \mathbf{E}_{z} & -\mathbf{R}_{j} \mathbf{E}_{z} \mathbf{R}_{j}
\end{array}\right]
$$

Bei der Rückstellkraft (Gl. B.92) werden die Momente durch das Kräftepaar von Gewichtskraft $G$ und Auftriebskraft $A$ (siehe Abb. B.8) nicht berücksichtigt, die bei Roll- und Stampfbewegungen $\left(s_{4}\right.$ und $s_{5}$ ) auftauchen. Daher ist Gl. (B.92) nur gültig, wenn der Verdrängungsschwerpunkt $B$ mit dem Gewichtsschwerpunkt $S$ zusammenfällt. Gl. (B.92) muss daher durch eine zusätzliche Matrix $\Delta \mathbf{C}$ ergänzt werden. Bei

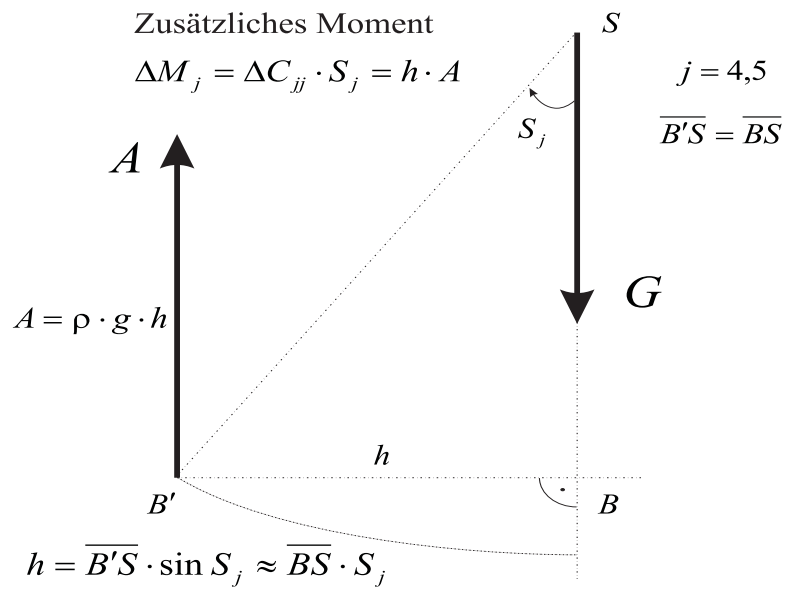

Abbildung B.8: Krängungsmoment durch Schwerpunktsverschiebung

schwimmenden Körpern besitzt sie nur zwei von Null verschiedene Elemente, für die bei kleinen Bewegungen gilt (Newman, 1977):

$$
\Delta c_{44}=\Delta c_{55}=\rho g V \overline{B S}
$$

wobei $V$ die Verdrängung der Struktur und $\overline{B S}$ der Abstand zwischen Verdrängungsschwerpunkt $B$ und Gewichtsschwerpunkt $S$ ist. Für die Rückstellkraft ergibt sich damit folgende endgültige Gleichung:

$$
\mathbf{F}_{c}^{*}=-[\mathbf{C}+\Delta \mathbf{C}] \mathbf{s}^{*}=-\mathbf{C}_{s} \mathbf{s}^{*}
$$




\section{B.4 Die äußere Kraft auf die Gesamtstruktur}

In vorigen Abschnitten wurden aus der verallgemeinerten Morisongleichung die äußere Kraft und das äußere Moment auf ein Strukturelement berechnet. Die gesamte äußere Kraft ist gleich der Summe aus hydrodynamischer und hydrostatischer Kraft. Sie kann mit den Begriffen der Erregerkraft $\mathbf{F}_{e}^{*}$, der Matrix der hydrodynamischen Massen A, der Dämpfungsmatrix B und der Matrix der Rückstellkoeffizienten $\mathbf{C}$ zusammengefasst werden:

$$
\mathbf{F}_{a}^{*}=\mathbf{F}_{e}^{*}-\mathbf{A} \frac{\partial \mathbf{u}^{*}}{\partial t}-\mathbf{B u}^{*}-\mathbf{C}_{s} \mathbf{s}^{*}
$$

Dabei gilt für $\mathbf{F}_{e}^{*}$ folgende Beziehung:

$$
\begin{aligned}
\mathbf{F}_{e}^{*}= & \left(1+C_{a}\right) \mathbf{F}_{F K N}^{*}+\mathbf{F}_{p}^{*} \\
& +\frac{\rho}{2} \frac{8}{3 \pi} C_{d}\left[\begin{array}{cc}
\mathbf{T}_{N} & \mathbf{0} \\
\mathbf{X}_{0 s} \mathbf{T}_{N} & \mathbf{E}_{T} \mathbf{T}_{N}
\end{array}\right]\left[\begin{array}{c}
\mathbf{I}_{D v} \\
\mathbf{I}_{D v \ell}
\end{array}\right]
\end{aligned}
$$

Die Erregerkraft besteht aus Termen, die von Größen der ungestörten Wellenströmung abhängen. $\mathbf{F}_{p}^{*}$ ist eine Funktion des dynamischen Druckes $p_{d y n}$ und $\mathbf{F}_{F K N}^{*}$ hängt von der Wellenpartikelbeschleunigung $\partial v / \partial t$ ab (siehe Gl. B.22). Jedoch hängen die beiden Integrale $\mathbf{I}_{D v}$ und $\mathbf{I}_{D v \ell}$ nicht nur von der Wellenpartikelgeschwindigkeit $v$ ab, sondern auch von der Amplitude der Relativgeschwindigkeit $\left[\mathbf{u}_{R N}\right]_{a}$, die mit den unbekannten Bewegungsvektoren gekoppelt ist (siehe Gl. B.42). Deshalb ist die Erregerkraft nicht von der Körperbewegung unabhängig und kann somit nicht im voraus ermittelt werden.

Die gesuchte äußere Kraft auf die Gesamtstruktur lässt sich durch komplexe Addition (wegen der Phasenbeziehungen) der Einzelkräfte $\mathbf{F}_{e}^{*}$ (Gl. B.96) ermitteln. Dabei werden Wechselwirkungen mit benachbarten Bauteilen nicht berücksichtigt. Im realen Fall liegen komplizierte dreidimensionale Strömungszustände vor, die durch dieses einfache Modell der Struktur nicht erfasst werden.

\section{B.5 Die Bewegungsdifferentialgleichungen}

Die Bewegungsdifferentialgleichungen für einen starren Körper lassen sich mittels Schwerpunktund Drallsatz der Mechanik bestimmen. Daraus erhält man ein Differentialgleichungssystem mit 6 Gleichungen:

$$
\mathbf{M} \frac{\partial \mathbf{u}^{*}}{\partial t}=\mathbf{F}_{a}^{*}
$$

mit der Matrix der Massenträgheitsmomente $\mathbf{M}$ :

$$
\mathbf{M}=\left[\begin{array}{cccccc}
m & 0 & 0 & 0 & 0 & 0 \\
0 & m & 0 & 0 & 0 & 0 \\
0 & 0 & m & 0 & 0 & 0 \\
0 & 0 & 0 & m_{44} & m_{45} & m_{46} \\
0 & 0 & 0 & m_{54} & m_{55} & m_{56} \\
0 & 0 & 0 & m_{64} & m_{65} & m_{66}
\end{array}\right]
$$


Setzt man die Gl. (B.96) für die äußere Kraft in die Bewegungsgleichungen (B.98) ein und bringt alle von der Schwerpunktsbewegung abhängigen Terme (bis auf die Erregerkraft) auf die linke Seite, erhält man ein lineares, inhomogenes Differentialgleichungssystem 2.Ordnung:

$$
(\mathbf{M}+\mathbf{A}) \frac{\partial \mathbf{u}^{*}}{\partial t}+\mathbf{B u}^{*}+\mathbf{C}_{s} \mathbf{s}^{*}=\mathbf{F}_{e}^{*}
$$

Zur Lösung des Differentialgleichungssystems wird für die Erregerkraft $\mathbf{F}_{e}^{*}$ und für die Körperbewegung $\mathbf{s}^{*}$ jeweils folgender Ansatz mit der harmonischen Funktion der Zeit gemacht:

$$
\begin{aligned}
\mathbf{F}_{e}^{*} & =\mathbf{F}_{e A}^{*} \exp (-i \omega t) \\
\mathbf{s}^{*} & =\mathbf{s}_{A}^{*} \exp (-i \omega t)
\end{aligned}
$$

Für die Körperbewegung wird eine harmonische Funktion gleicher Frequenz wie für die Erregerkraft, aber mit einer zusätzlichen Phasenverschiebung $\varepsilon$ angesetzt. Daher gilt für die komplexe Amplitude $\mathbf{s}_{A}^{*}$ folgende Beziehung:

$$
\mathbf{s}_{A}^{*}=\mathbf{s}_{a}^{*} \exp (-i \varepsilon)
$$

Die ersten beiden zeitlichen Ableitungen von $\mathbf{s}^{*}$, Geschwindigkeit $\mathbf{u}^{*}$ und Beschleunigung $\partial \mathbf{u}^{*} / \partial t$ lauten dann:

$$
\begin{aligned}
\mathbf{u}^{*} & =-i \omega \mathbf{s}_{A}^{*} \exp (-i \omega t) \\
\frac{\partial \mathbf{u}^{*}}{\partial t} & =-\omega^{2} \mathbf{s}_{A}^{*} \exp (-i \omega t)
\end{aligned}
$$

Durch Einsetzen der Gleichungen (B.101) und (B.103) sowie Division durch den zeitabhängigen Faktor $\exp (-i \omega t)$ erhält man ein lineares Gleichungsystem für die komplexe Amplitude $\mathbf{s}_{A}^{*}$ der Schwerpunktsbewegung:

$$
\left[\mathbf{C}-i \omega \mathbf{B}-\omega^{2}(\mathbf{M}+\mathbf{A})\right] \mathbf{s}_{A}^{*}=\mathbf{F}_{e A}^{*}
$$

\section{B.6 Die Berechnung der Bewegungsübertragungsfunk- tion}

Das Gleichungssystem (B.104) enthält als linearen Faktor die Wellenamplitude $\zeta_{a}$. Um die gesuchte Übertragungsfunktion der Bewegung zu berechnen, wird das Gleichungssystem (B.104) auf die Wellenamplitude normiert. Daraus ergibt sich für die Übertragungsfunktion $\mathbf{H}^{*}(\omega)$ (Siehe Gl. 5.4) :

$$
\begin{gathered}
{\left[\mathbf{C}-i \omega \mathbf{B}-\omega^{2}(\mathbf{M}+\mathbf{A})\right] \mathbf{H}^{*}=\mathbf{f}_{e A}^{*}} \\
\operatorname{mit} \quad \mathbf{f}_{e A}^{*}=\frac{\mathbf{F}_{e A}^{*}}{\zeta_{a}}
\end{gathered}
$$

Die Matrizen A und $\mathbf{C}$ müssen nur einmal für die Struktur berechnet werden. Die normierte Erregerkraft $\mathbf{f}_{e A}^{*}$ und die Dämpfungsmatrix $\mathbf{B}$ sind, wie bereits diskutiert, 
sowohl frequenzabhängig als auch eine Funktion von der gesuchten, unbekannten Körperbewegung. Die gesuchte komplexe Übertragungsfunktion kann daher nicht direkt, sondern nur iterativ ermittelt werden.

Um das gekoppelte Gleichungssystem (B.105) iterativ zu lösen, wird zuerst ein Startvektor $\mathbf{H}^{*(0)}$ eingesetzt, der nur den Nullwert enthält:

$$
\mathbf{H}^{*(0)}=\left[\begin{array}{c}
\widetilde{\mathbf{s}}_{T} \\
\widetilde{\mathbf{s}}_{D}
\end{array}\right]=\left[\begin{array}{l}
\mathbf{0} \\
\mathbf{0}
\end{array}\right]
$$

Man stellt sich hierbei vor, dass die Ruhelage des Systems zunächst die Lösung ist. Setzt man den Startvektor in Gln. (B.97) und (B.51) ein, erhält man die erste Näherung für die Erregerkraft $\mathbf{f}_{e A}^{*(0)}$ und die Dämpfungsmatrix $\mathbf{B}^{(0)}$. Da die Matrizen und die Erregerkraft bekannt sind, lässt sich nun der 1. iterierte Vektor $\mathbf{H}^{*(1)}$ aus dem linearen Gleichungssystem (B.105) ermitteln:

$$
\mathbf{H}^{*(1)}=\left[\mathbf{C}-i \omega \mathbf{B}^{(0)}-\omega^{2}(\mathbf{M}+\mathbf{A})\right]^{-1} \mathbf{f}_{e A}^{*(0)}
$$

Die inverse Matrix kann dabei durch bekannte Inversionsverfahren, wie Links-RechtsZerlegung berechnet werden. Der 1. iterierte Vektor $\mathbf{H}^{*(1)}$ wird dann wieder als bekannte Lösung für die weitere Vektor-Iteration verwendet, wobei die Erregerkraft $\mathbf{f}_{e A}^{*(1)}$ und der Dämpfungsmatrix $\mathbf{B}^{(1)}$ im voraus berechnet werden. Analog zu Gl. (B.107) erhält man den 2. iterierten Vektor $\mathbf{H}^{*(2)}$ :

$$
\mathbf{H}^{*(2)}=\left[\mathbf{C}-i \omega \mathbf{B}^{(1)}-\omega^{2}(\mathbf{M}+\mathbf{A})\right]^{-1} \mathbf{f}_{e A}^{*(1)}
$$

und den n-ten iterierten Vektor $\mathbf{H}^{*(n)}$ :

$$
\mathbf{H}^{*(n)}=\left[\mathbf{C}-i \omega \mathbf{B}^{(n-1)}-\omega^{2}(\mathbf{M}+\mathbf{A})\right]^{-1} \mathbf{f}_{e A}^{*(n-1)}
$$

Die Vektor-Iteration wird solange durchgeführt, bis die Lösung mit ausreichender Genauigkeit konvergiert. Hierzu wird folgendes Kriterium definiert, in dem zur Fehlerabschätzung des Vektors die Euklidische Norm verwendet wurde:

$$
\frac{\left\|\mathbf{H}^{*(n)}-\mathbf{H}^{*(n-1)}\right\|}{\left\|\mathbf{H}^{*(n-1)}\right\|}<10^{-6}
$$




\section{B.7 Das Programm TFC (Transfer Function Calcu- lation)}

Aus den abgeleiteten Gleichungen wurde das Programm TFC (Transfer Function Calculation) entwickelt. Das Programm TFC enthält neben verschiedenen Modulen für die Ein- und Ausgabe als zentralen Teil ein Modul zur Berechnung der Übertragungsfunktion. Abb. B.9 zeigt den prinzipiellen Ablauf des Programmes.

$\mathrm{Zu}$ Beginn werden die Eingabedateien für die Struktur und Wellen eingelesen. Anhand von gegebenen Formparametern wird dann die Spantarealkurve bestimmt. Nach der Geometrieverarbeitung erfolgt die Berechnung der Matrix der hydrodynamischen Massen A und der hydrostatischen Rückstellkoeffizienten C. Die Matrix C ist von den Wellenperioden unabhängig.

Bei der Berechnung der frequenzabhängigen Größen (Wellenkräfte, Übertragungsfunktionen) muss die Wellenzahl vorab für jede durch das Eingabefile spezifizierten Wellenperiode berechnet werden. Die Wellenzahl $\kappa$ wird aus der transzendenten Gleichung

$$
\kappa=\frac{\omega^{2}}{g \cdot \tanh (\kappa d)}
$$

durch ein Newton-Iterationsverfahren gelöst. Die mit der unbekannten Körperbewegung gekoppelte Erregerkraft sowie die Dämpfungsmatrix werden mit dem Nullvektor als Startvektor iterativ ermittelt. Bei jeder Iteration wird das lineare, komplexe Gleichungssystem neu erstellt und durch Links-Rechtszerlegung gelöst. Die Iteration wird beendet, wenn die Differenz zwischen zwei aufeinanderfolgenden Beträgen des iterierten Lösungsvektors kleiner als $10^{-6}$ ist. Die Konvergenz der Lösung ist von der Wellenperiode abhängig. Im Bereich der Resonanz- und der Auslöschungsfrequenz, bei denen die Zähigkeitsdämpfung eine Rolle spielt, benötigt die Berechnung mehr Iterationsschritte. Im übrigen Frequenzbereich konvergiert die Lösung schnell.

Um den Zähigkeitseinfluss auf das Bewegungsverhalten zu veranschaulichen, wird im folgenden der Betrag der Übertragungsfunktion der Tauchbewegung betrachtet. Nach Gl. (B.105) ergibt sich für $s_{3 a} / \zeta_{a}$

$$
\begin{array}{r}
\frac{s_{3 a}}{\zeta_{a}}=\frac{\mathbf{F}_{3 e A}}{c_{33} \zeta_{a} \sqrt{\left[1-\left(\frac{\omega}{\omega_{R}}\right)^{2}\right]^{2}+\left(\frac{\omega b_{33}}{c_{33}}\right)^{2}}} \\
\text { mit } \quad \omega_{R}=\sqrt{\frac{c_{33}}{m_{33}+a_{33}}}
\end{array}
$$

Bei der Auslöschungsfrequenz $\omega=\omega_{C}$ verschwindet die Trägheitskraft, so dass die Wellenkraft auf die Struktur ausschließlich aus der geschwindigkeitsabhängigen Zähigkeitskraft besteht. Die Widerstandskraft führt zu endlichen Bewegungen im Bereich der Auslöschungsfrequenz (siehe z.B. Abb. B.10). Für die Resonanzfrequenz $\omega=\omega_{R}$ reduziert sich der Nenner auf das Glied $b_{33} \zeta_{a} \omega_{R}$, hängt also ebenfalls von der Dämpfungsmatrix ab. Der von der Wellenamplitude abhängige Dämpfungskoeffizient $b_{33}$ begrenzt im Resonanzfall die Bewegungsamplitude auf endliche Werte. 


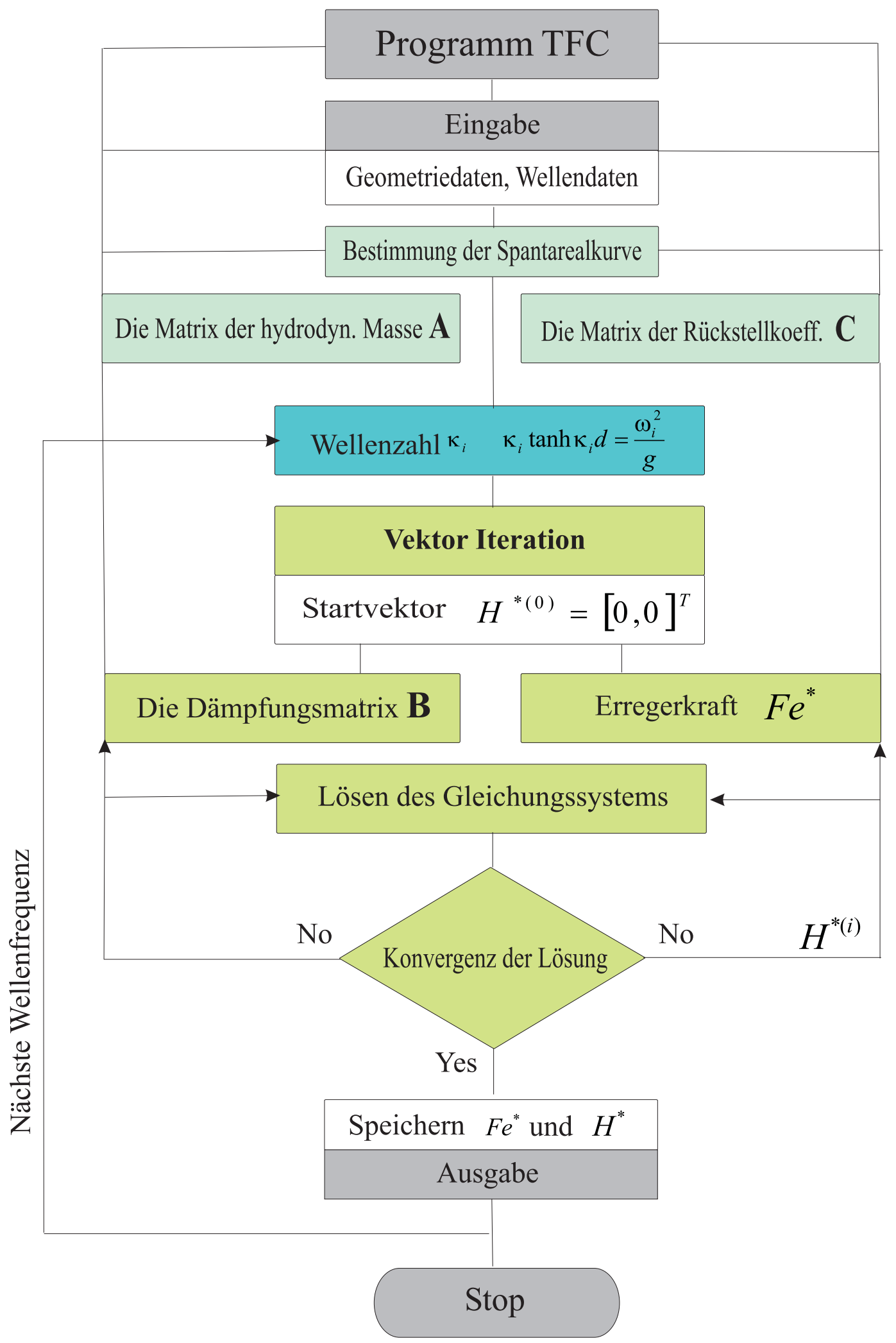

Abbildung B.9: Schematischer Ablauf des Programmes TFC 


\section{B.8 Validierung des Programmes mit Versuchdaten}

Um das entwickelte Programm zu validieren, werden in diesem Abschnitt die Übertragungsfunktionen der Bewegung für zwei Modelle eines Halbtauchers mit gemessenen Versuchdaten verglichen (Birk, 1990b, 1991).
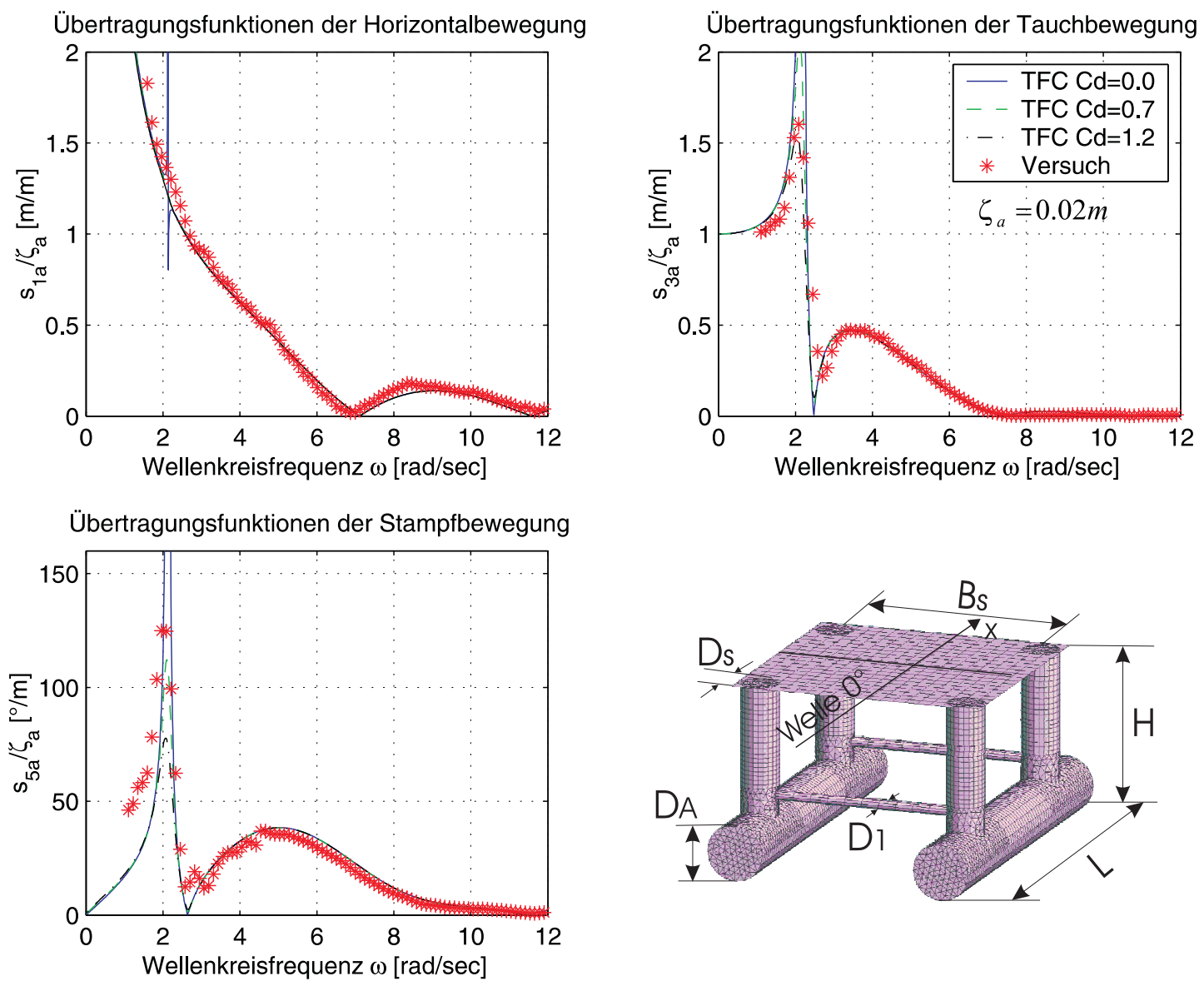

Abbildung B.10: Vergleich der gerechneten und experimentellen Übertragungsfunktionen bei einem Welleneinfallswinkel von $\varphi=0^{\circ}$.

Die Abbildungen B.10, B.11 und B.12 zeigen die Übertragungsfunktionen der Bewegung für das erste Halbtauchermodell. Das Modell besteht aus Zylindern mit konstanten Querschnittsflächen und hat folgende Konstruktionsdaten (Birk, 1991):

$$
\begin{array}{ccc}
\mathrm{m}=49.76 \mathrm{~kg} & \mathrm{~L}=114.5 \mathrm{~cm} & D_{A}=15.0 \mathrm{~cm} \\
D_{S}=12.0 \mathrm{~cm} & D_{1}=3.0 \mathrm{~cm} & \mathrm{H}=56.9 \mathrm{~cm}
\end{array}
$$

Bei allen untersuchten Welleneinfallswinkeln $\left(\varphi=0^{\circ}, 45^{\circ}\right.$ und $\left.90^{\circ}\right)$ ergibt sich für Horizontal- und Vertikalbewegung eine sehr gute Übereinstimmung der Ergebnisse. Im Bereich der Tauchresonanz bei $\omega \approx 2.1[\mathrm{rad} / \mathrm{s}]$ spielt wie erwartet die Zähigkeitsdämpfung eine Rolle, wobei das Maximum der Tauchbewegung für den Widerstandsbeiwert $C_{d}=1.2$ am besten mit den experimentellen Ergebnissen übereinstimmt. Resonanzund Auslöschungsfrequenz werden von der Rechnung sehr gut wiedergegeben. 

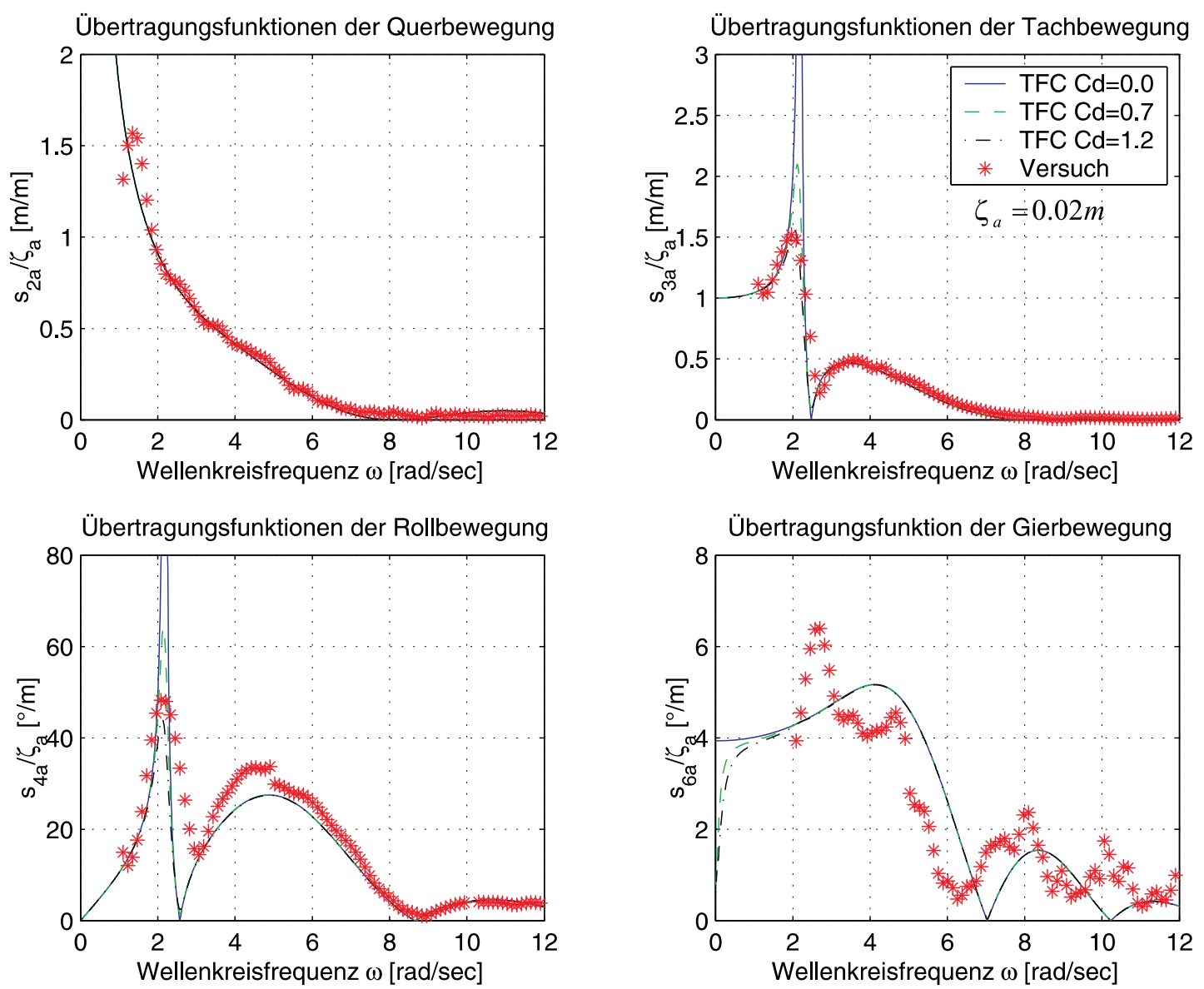

Abbildung B.11: Vergleich der gerechneten und experimentellen Übertragungsfunktionen bei einem Welleneinfallswinkel von $\varphi=45^{\circ}$.

Auch für die rotatorischen Bewegungen liefert das Programm gute Ergebnisse. Bei Stampfbewegung (siehe Abb. B.10 unten links) ergibt sich eine gute Übereinstimmung der Resonanzfrequenz $(\omega \approx 2.1[\mathrm{rad} / \mathrm{s}])$ und der Auslöschungsfrequenz $(\omega \approx 2.7$ $[\mathrm{rad} / \mathrm{s}])$. Das Maximum der berechneten Stampfbewegung bei Resonanzfrequenz ist für $C_{d}=1.2$ etwas kleiner als das gemessene. Im mittleren und oberen Frequenzbereich stimmen die numerischen und experimentellen Ergebnisse gut überein. Ähnliches gilt auch für die Rollbewegung (siehe Abbildungen B.11 und B.12 unten links). Die Resonanzpeaks der Rollbewegung sind niedriger als bei Stampfbewegung. Die Gierbewegung (siehe Abb. B.11 unten rechts) ist nahezu eine Größenordnung kleiner als die Rollbewegung. Für $\omega<2[\mathrm{rad} / \mathrm{s}]$ wächst der Betrag der experimentell ermittelten Übertragungsfunktion stark an. Die Ursache dafür ist eine Folge der Resonanzschwingung des Modells im Verankerungssystem. Im Frequenzbereich $\omega>2[\mathrm{rad} / \mathrm{s}]$ stimmen die numerischen und experimentellen Ergebnisse gut überein.

Abb. B.13 zeigt einen Caisson-Halbtaucher, der aus vier Säulen mit veränderlichen Querschnittsflächen besteht. Das Seegangsverhalten dieser für ein Pierson-MoskowitzSpektrum mit $T_{0}=9 \mathrm{~s}$ optimierten Struktur wurde durch Modellversuche im Maßstab 1:70 verifiziert (Heimann, 1993). Kräfte und Bewegungen wurden in transienten Wel- 

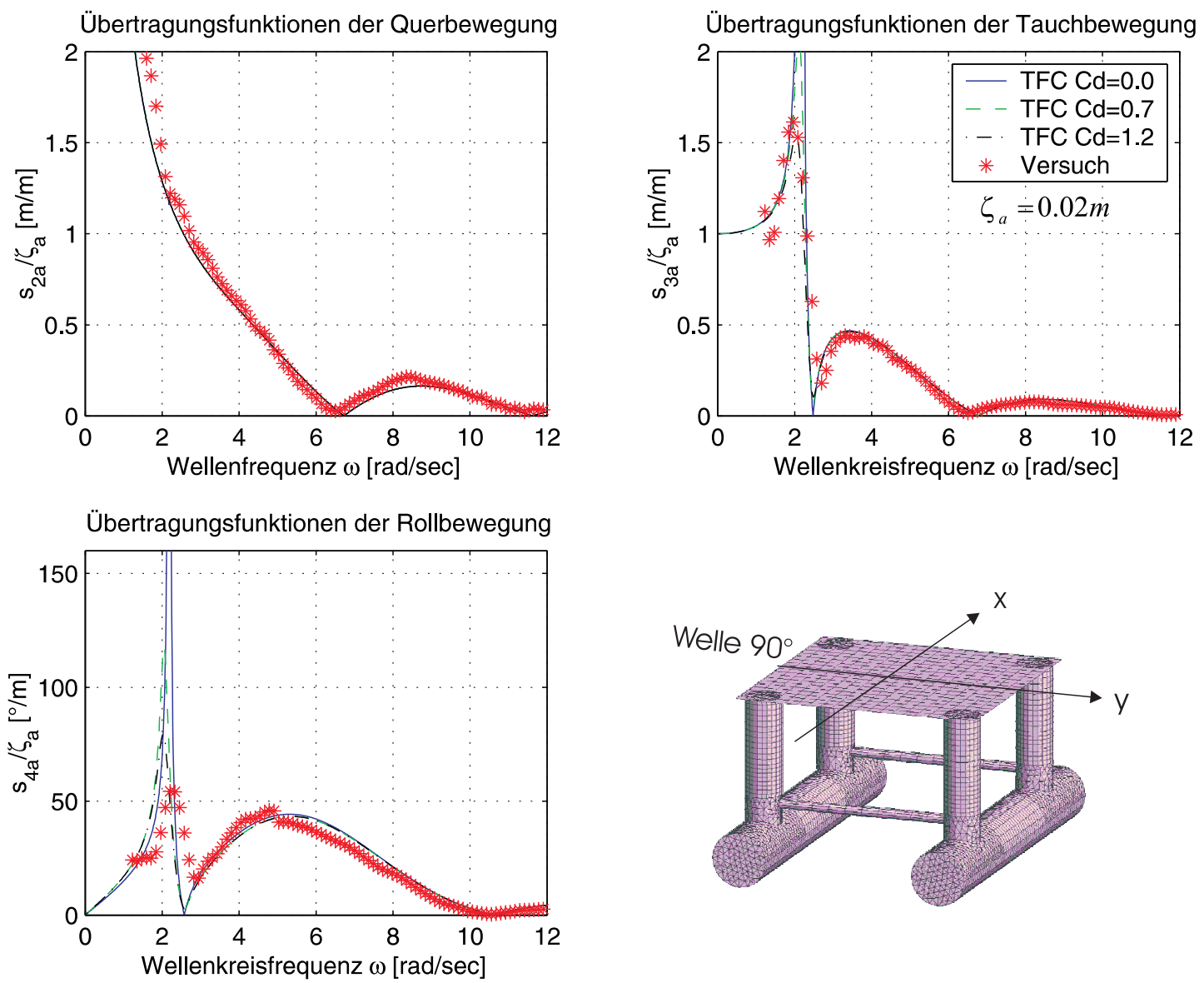

Abbildung B.12: Vergleich der gerechneten und experimentellen Übertragungsfunktionen bei einem Welleneinfallswinkel von $\varphi=90^{\circ}$.

lenpaketen gemessen (Clauss und Kühnlein, 1994). Wie die Übertragungsfunktionen für die Tauchbewegung in Abb. B.13 zeigen, stimmen die numerischen Ergebnisse mit den experimentellen Daten gut überein. Für Strukturen mit Caisson-artigen Auftriebskörpern darf die hydrodynamische Masse in Richtung der Zylinderachse nicht vernachlässigt werden. Für den untersuchten Caisson-Halbtaucher lässt sich die hydrodynamische Masse $a_{33}$ in Axialrichtung nach dem Näherungsansatz (Gl. B.70) berechnen. Sie beträgt nahezu ein Drittel der Verdrängung $\left(a_{33}=9973.8 t\right)$. Mit dem Rückstellkoeffizient $c_{33}=4 \rho g A_{W L}=3002 \mathrm{kN} / \mathrm{m}$ lässt sich die Resonanzfrequenz nach Gl. (B.113) bestimmen:

$$
\omega_{R}=\sqrt{\frac{c_{33}}{\rho V+a_{33}}}=0.274 s^{-1}
$$

Dies liegt genau auf dem experimentell ermittelten Wert, wodurch die zuverlässige Vorhersage der Übertragungsfunktionen auch für Strukturen mit veränderlichen Querschnitten bestätigt wird. 

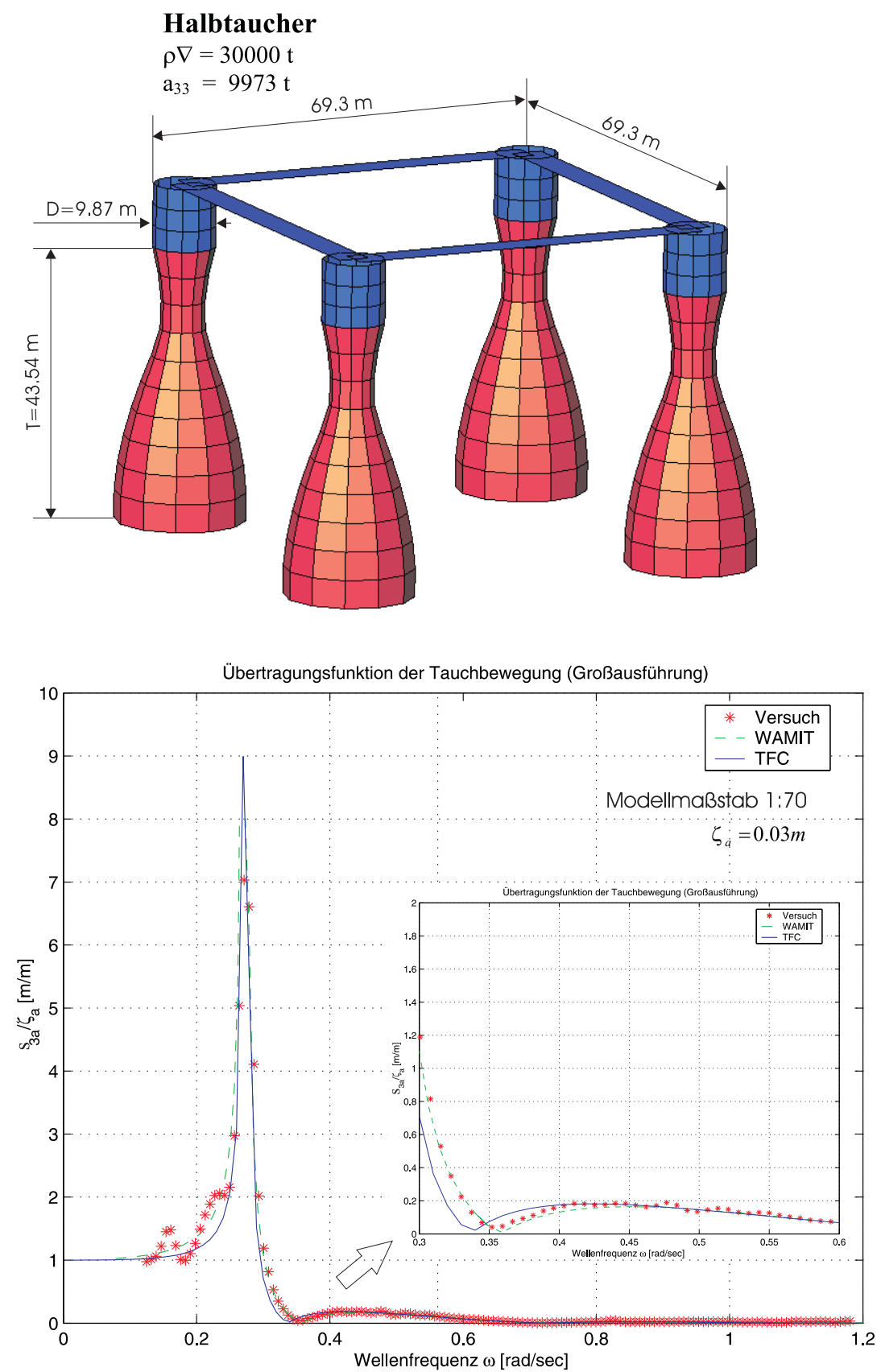

Abbildung B.13: Vergleich der gerechneten und experimentellen Übertragungsfunktionen am Beispiel eines Caisson-Halbtauchers mit veränderlichen Querschnittsflächen: Welleneinfallswinkel $\varphi=0^{\circ}$. 


\section{Anhang C}

\section{Grundlagen des Programmsystems WAMIT}

Hydrodynamische kompakte Strukturen beeinflussen das Initialwellenfeld erheblich. Man unterscheidet zwei Effekte:

- Diffraktion und

- Radiation

Im allgemeinen bezeichnet man beide Effekte zusammen als Diffraktion (engl. diffraction). Das Wort Diffraktion kommt aus dem lateinischen und bedeutet Beugung von Wellen. Ein ruhender Körper im Initialwellenfeld wird dieses also durch Beugung verändern (Diffraktion). Ein Teil der Initialwelle wird dabei auch reflektiert. Ein Körper, der sich in ruhendem Wasser bewegt, strahlt Wellen ab (Radiation). Die für die Erzeugung der Wellen notwendige Energie wird der kinematischen Energie der Struktur entzogen, d.h. die Bewegung wird gedämpft. Dieser Energieverlust wird als Potentialdämpfung bezeichnet. In großer Entfernung vom Körper sind die Radiationswellen immer kreisförmig.

Bei hydrodynamischen kompakten Strukturen überwiegen die Trägheitskräfte und können im Rahmen einer linearen Theorie zähigkeitsbedingte Effekte vernachlässigt werden. Das Verhältnis von Wellenamplitude $\zeta_{a}$ und charakteristischer Abmessung $D$ des Körpers ist klein, d.h. $\zeta_{a} / D \ll 1$. Dadurch ergeben sich kleine Geschwindigkeitsgradienten, bzw. kleine Schubspannungen an der Körperoberfläche. Es wird deshalb im folgenden von einer idealen, rotationsfreien Flüssigkeit ausgegangen.

\section{C.1 Das Randwertproblem für einen Körper im Wel- lenfeld}

Unter der Voraussetzung von der idealen Flüssigkeit lässt sich die Strömung um einen Körper im langkämmigen Wellenfeld durch ein Potential $\phi$ beschrieben werden. Aus dem Potential $\phi$ lassen sich Strömmungsgeschwindigkeiten und Drücke bestimmen, mit 
denen dann alle anderen dynamischen Größen berechnet werden können. Das Potential $\phi$ muss die bekannte Laplacegleichung erfüllen:

$$
\Delta \phi=0
$$

Unter der Voraussetzung der Linearität des Problems wird für das Gesamtpotential $\phi$ folgende Ansatz gemacht (Newman, 1977):

$$
\phi=\phi_{0}+\phi_{7}+\sum_{l=1}^{6} \phi_{l}
$$

Dabei ist

- $\phi_{0}$ das Potential der ungestörten Welle,

- $\phi_{7}$ das Diffraktionspotential, das die Störung der einfallenden Welle durch den unbewegten Körper beschreibt, und

- $\phi_{l}=\dot{s}_{l} \varphi_{l}$ die den sechs Freiheitsgraden der Starrrkörperbewegung zugeordneten Radiationspotentiale. Sie beschreiben die durch die jeweilige Körperbewegung verursachte Wellenabstrahlung. $\underline{\dot{s}}=\left(\dot{s}_{1} \dot{s}_{2}, \ldots \dot{s}_{6}\right)^{T}$ ist der generalisierte Geschwindigkeitsvektor des Körpers ${ }^{1}$. Die Ortsfunktion $\varphi_{l}$ beschreiben das Potential aufgrund einer Einheitsgeschwindigkeit für einen der Freiheitsgrade $l=1 \ldots 6$.

Die gesuchte Lösung der Laplacegleichung muss zusätzlich die linearisierten Randbedingungen erfüllen (Abb. C.1):

(i) am Meeresboden $S_{B}(z=-d)$ :

$$
\frac{\partial \phi}{\partial z}=0
$$

(ii) an der freien Flüssigkeitsoberfläche $S_{F}(z=0)$ :

$$
\frac{\partial \phi}{\partial z}-\frac{\omega^{2}}{g} \phi=0
$$

(iii) auf der benetzten Körperoberfläche $S_{b}$ :

$$
\frac{\partial \phi}{\partial n}=\underline{\dot{s}}^{T} \underline{n}
$$

(iv) und im Fernfeld $S_{\infty}$ (Sommerfeld'sche-Ausstrahlungsbedingung):

$$
\begin{array}{r}
\lim _{R \rightarrow \infty} \sqrt{R}\left(\frac{\partial \phi_{i}}{\partial R}-i k \phi_{j}\right)=0 j=1, \ldots, 7 \\
\operatorname{mit} R=\sqrt{(x-\xi)^{2}+(y-\eta)^{2}}
\end{array}
$$

\footnotetext{
${ }^{1} \dot{s}_{1}, \dot{s}_{2}, \dot{s}_{3}$ stehen für die translatorischen und $\dot{s}_{4}, \dot{s}_{5}, \dot{s}_{6}$ für die rotatorischen Geschwindigkeiten
} 


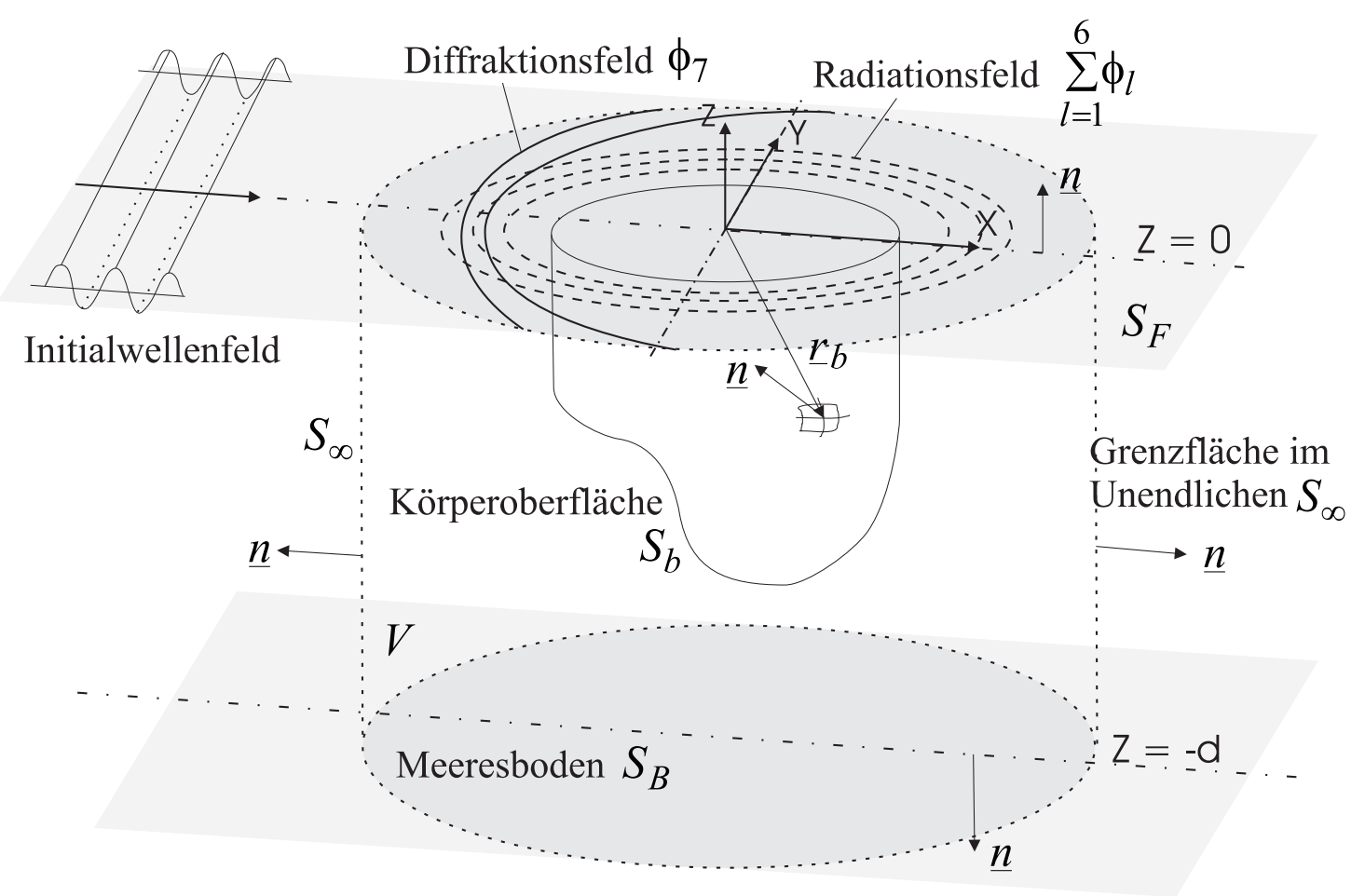

Abbildung C.1: Schematische Darstellung des Diffraktion-Radiationsproblems

Der Normalenvektor $\underline{n}$ der benetzten Körperoberfläche zeigt aus dem Flüssigkeitsbereich heraus, also in den Körper hinein. Der generalisierte Normalenvektor $\underline{n}^{*}$ besteht aus den translatorischen und den rotatorischen Komponenten aus, d.h.

$$
\underline{n}^{*}=\left(\begin{array}{c}
\underline{n} \\
\underline{r}_{b} \times \underline{n}
\end{array}\right)
$$

Die kinematische Randbedingung auf der benetzten Körperoberfläche verlangt, dass die lokale Strömungsgeschwindigkeit normal zur Körperoberfläche $\partial \phi / \partial n$ gleich der lokalen Punktgeschwindigkeit der Körperoberfläche $\underline{\dot{s}}^{T} \underline{n}$ ist. Daraus folgt für einen feststehenden Körper $(\underline{\dot{s}} \equiv 0)$ im Wellenfeld

$$
\frac{\partial \phi_{0}}{\partial n}=-\frac{\partial \phi_{7}}{\partial n}
$$

und für einen bewegten Körper in ruhigem Wasser

$$
\frac{\partial \phi}{\partial n}=\underline{\dot{s}}^{T} \underline{n}^{*} \text { oder } \frac{\partial \varphi_{l}}{\partial n}=\underline{n}^{*} \quad \text { mit } l=1,2, \ldots, 6 .
$$

Im Fall der bewegten Struktur im Wellenfeld gelten natürlich beide Bedingungen (C.8) und (C.9) gleichzeitig.

Die Lösung des Randwertproblems, das durch die Laplacegleichung (C.1) und die Randbedingungen (C.3 - C.6) definiert wird, beruht auf dem 2. Greenschen Satz

$$
\iint_{V} \int[\phi \Delta G-G \Delta \phi] d V=\iint_{S}\left[\phi \frac{\partial G}{\partial n}-G \frac{\partial \phi}{\partial n}\right] d S .
$$


Durch die Anwendung des Greenschen Satzes (C.10) wird das ursprünglich dreidimensionale Problem auf zwei Dimensionen reduziert. Die sogenannte Greensche Funktion $G(\underline{x}, \underline{\xi})$ beschreibt das Potential im Punkt $\underline{x}=(x, y, z)^{T}$ aufgrund einer harmonisch pulsierenden Quelle (Singularität) der Stärke $-4 \pi$ im Punkt $\underline{\xi}=(\xi, \eta, \zeta)^{T}$. Die Greensche Funktion setzt sich aus einer Rankinequelle $1 / r$ und einer regulären Funktion $H(\underline{x}, \underline{\xi})$ zusammen, die beide für sich die Laplacegleichung erfüllen:

$$
\begin{array}{r}
G(\underline{x}, \underline{\xi})=\frac{1}{r}+H(\underline{x}, \underline{\xi}) \\
\text { mit } r=\sqrt{(x-\xi)^{2}+(y-\eta)^{2}+(z-\zeta)^{2}}
\end{array}
$$

Details über die Herleitung der Greenschen Funktion finden sich bei Wehausen und Laitone (1960). Offensichtlich ist die Greensche Funktion singulär für $\underline{x}=\underline{\xi}$ (Newman, 1977).

Das Oberflächenintegral in Gl. (C.10) umfasst die gesamte Berandung des Strömungsgebietes, d.h. Meeresboden, freie Wasseroberfläche, benetzte Körperoberfläche und die Grenzfläche im Fernfeld. Setzt man alle oben genannten Randbedingungen in Gl. (C.10) ein und berücksichtigt, dass $\phi$ und $G$ die Laplacegleichung erfüllen, reduziert sich das Oberflächenintegral auf die benetzte Körperoberfläche. Alle anderen Anteile werden identisch Null (Mei, 1989). Aufgrund der Singularität im Punkt $\underline{x}=\underline{\xi}$ muss das verbleibende Integral in zwei Teilintegrale aufgespalten werden:

- Die Punktquelle auf der benetzten Körperoberfläche wird durch eine Halbkugel $S_{h s}$ mit Radius $R$ umfasst. Mit $d S=R^{2} d \alpha d \beta$ und $\partial / \partial n=-\partial / \partial R$ ergibt sich für das Integral über die Halbkugel ${ }^{2}$ für $R \rightarrow 0$ :

$$
\iint_{S_{h s}}\left[\phi(\underline{x}) \frac{\partial}{\partial n} \frac{1}{R}-\frac{1}{R} \frac{\partial \phi(\underline{x})}{\partial n}\right] d S=2 \pi \phi(\underline{x}) .
$$

- Auf dem verbleibenden Teil der benetzten Fläche $S_{b}$ (ohne die Singularität) ist die Greensche Funktion regulär, die Integration also problemlos möglich. Das Volumenintegral in Gl. (C.10) verschwindet identisch zu Null. Mit Gl. (C.12) erhält man demnach für das Integral über die gesamte benetzte Oberfläche

$$
2 \pi \phi(\underline{x})+\iint_{S_{b}}\left[\phi(\underline{\xi}) \frac{\partial G(\underline{x}, \underline{\xi})}{\partial n}-G(\underline{x}, \underline{\xi}) \frac{\partial \phi(\underline{\xi})}{\partial n}\right] d S=0 .
$$

Somit folgt mit Gln. (C.12) und (C.13) aus dem 2. Greenschen Satz (C.10) die Integralgleichung

$$
\begin{array}{r}
2 \pi \phi(\underline{x})+\iint_{S_{b}} \phi(\underline{\xi}) \frac{\partial G(\underline{x}, \underline{\xi})}{\partial n} d S=\iint_{S_{b}} G(\underline{x}, \underline{\xi}) \frac{\partial \phi(\underline{\xi})}{\partial n} d S \\
\left(\underline{x}, \underline{\xi} \in S_{b} \text { und } \underline{x} \neq \underline{\xi}\right) .
\end{array}
$$

\footnotetext{
${ }^{2}$ Die Integration ergibt $4 \pi \phi(\underline{x})$ wenn die Singularität im Strömungsgebiet $V$ liegt und in eine Kugel eingeschlossen werden muss.
} 
Gl. (C.14) gilt für jede beliebige Pontentialfunktion, insbesondere auch für die unbekannten Potentiale der rechten Seite von Gl. (C.2). Setzt man die Beziehungen (C.8) und (C.9) aus der kinematischen Randbedingung in Gl. (C.14) ein, erhält man sieben lineare Fredholmsche Integralgleichungen für die sechs Ortsfunktionen $\varphi_{l}$ (und damit die sechs Radiationspotentiale $\phi_{l}$ ) und für das Diffraktionspotential $\phi_{7}$ :

$$
\begin{aligned}
2 \pi \varphi_{l}(\underline{x})+\iint_{S_{b}} \varphi_{l}(\underline{\xi}) \frac{\partial G(\underline{x}, \underline{\xi})}{\partial n} d S=\iint_{S_{b}} n_{l} G(\underline{x}, \underline{\xi}) d S \\
2 \pi \phi_{7}(\underline{x})+\iint_{S_{b}} \phi_{7}(\underline{\xi}) \frac{\partial G(\underline{x}, \underline{\xi})}{\partial n} d S=-\iint_{S_{b}} G(\underline{x}, \underline{\xi}) \frac{\partial \phi_{0}(\underline{\xi})}{\partial n} d S
\end{aligned}
$$

Die rechte Seite von Gl. (C.16) erfordert eine aufwendige Integration. Durch Addition einer zusätzlichen Integralgleichung kann diese Integration jedoch umgangen werden. Betrachtet man die virtuelle Strömung im Inneren des Körpers - die benetzte Oberfläche stellt potentialtheoretisch nur eine Stromfläche und keine starre Wand dar - lässt sich analog zu den Gln. (C.15) und (C.16) die Integralgleichung

$$
-2 \pi \phi_{0}(\underline{x})+\iint_{S_{b}} \phi_{0}(\underline{\xi}) \frac{\partial G(\underline{x}, \underline{\xi})}{\partial n} d S=\iint_{S_{b}} G(\underline{x}, \underline{\xi}) \frac{\partial \phi_{0}(\underline{\xi})}{\partial n} d S
$$

ableiten. Das negative Vorzeichen folgt dabei aus dem in das betrachtete Gebiet hineinzeigenden Normalenvektor $\underline{n}$. Addiert man auf beiden Seiten der Gl. (C.17) den Term $4 \pi \phi_{0}$ und addiert das Ergebnsi zu Gl. (C.16) erhält man eine Integralgleichung mit einfacher rechter Seite. Führt man $\phi_{0}=\varphi_{0} e^{-i \omega t}$ sowie $\phi_{7}=\varphi_{7} e^{-i \omega t}$ ein und dividiert durch den Faktor $e^{-i \omega t}$, so ergibt sich abschließend eine Integralgleichung für das kombinierte Diffraktionspotential $\varphi_{D}=\varphi_{0}+\varphi_{7}$ :

$$
2 \pi \varphi_{D}(\underline{x})+\iint_{S_{b}} \varphi_{D}(\underline{\xi}) \frac{\partial G(\underline{x}, \underline{\xi})}{\partial n} d S=4 \pi \varphi_{0}(\underline{x})
$$

\section{C.2 Numerische Lösung}

Für die numerische Lösung des Geschwindigkeitspotential $\phi$ aus den Gln. (C.15) und (C.18) wird das Programmsystem WAMIT (1994) (Wave Analysis Massachusetts Institute of Technology) eingesetzt. Für die Diskretisierung der Integralgleichung wird angenommen, dass das Potential konstant auf jeder Panelfläche $S_{k}$ ist und der Mittelpunkt des Panels als Kontrollpunkt $\underline{x}_{k}$ verwendet wird. Somit ergeben sich aus den Integralgleichungen sieben lineare Gleichungssysteme. Die Anzahl der Unbekannten entspricht der Anzahl der für die Diskretisierung eingesetzten Panels $N_{P}$, d.h. es entsteht ein lineares Gleichungssystem mit $N_{P}$ Gleichungen für die Radiationspotentiale

$$
2 \pi \varphi_{l}\left(\underline{x}_{i}\right)+\sum_{k=1}^{N_{P}} \varphi_{l}\left(\underline{x}_{k}\right) \iint_{S_{k}} \frac{\partial G\left(\underline{x}_{i}, \underline{\xi}\right)}{\partial n} d S=\sum_{k=1}^{N_{P}} n_{l}^{*}\left(\underline{x}_{k}\right) \iint_{S_{k}} G\left(\underline{x}_{i}, \underline{\xi}\right) d S
$$


und für das kombinierte Diffraktionspotential

$$
2 \pi \varphi_{D}\left(\underline{x}_{i}\right)+\sum_{k=1}^{N_{P}} \varphi_{D}\left(\underline{x}_{k}\right) \iint_{S_{k}} \frac{\partial G\left(\underline{x}_{i}, \underline{\xi}\right)}{\partial n} d S=4 \pi \varphi_{0}\left(\underline{x}_{i}\right)
$$

die Bestimmung der Koeffizienten $D_{i k}$ und $S_{i k}$

$$
D_{i k}=\iint_{S_{k}} \frac{\partial G\left(\underline{x}_{i}, \underline{\xi}\right)}{\partial n} d S \quad \text { und } \quad S_{i k}=\iint_{S_{k}} G\left(\underline{x}_{i}, \underline{\xi}\right) d S .
$$

ist aufgrund der Greenschen Funktion $G(\underline{x}, \underline{\xi})$ sowie deren normalen Ableitung $\partial G(\underline{x}, \underline{\xi}) / \partial n$ die meist zeitraubende Operation bei der numerischen Lösung der Gl. (C.20). Die für die Auswertung der Integrale (C.21) verwendete Algorithmen finden sich bei Newman (1985, 1986). Die Genauigkeit der Lösung wird durch einen iterativen Gleichungslöser gewährleistet (Newman und Sclavounos, 1988). In der Regel wird eine Genauigkeit von sechs signifikanten Stellen erreicht. Um dem bei Integralgleichungen auftretenden Problem der irregular Frequenzen (John, 1950) zu begegnen, wurde die Methode von Lee und Sclavounos (1989) implementiert.

\section{C.3 Erregerkräfte und die Übertragungsfunktion der Bewegung}

Aus den Geschwindigkeitspotentialen lassen sich die zur Charakterisierung notwendigen physikalischen Größen ableiten. Erregerkräfte und -momente werden durch Integration des dynamischen Druckes über die benetzte Körperoberfläche berechnet. Der dynamische Druck $p$ auf die benetzte Körperoberfläche ergibt sich aus der linearisierten Bernoulli-Gleichung

$$
\begin{aligned}
p & =-\rho \frac{\partial \phi}{\partial t} \\
& =-\rho\left[\frac{\partial \phi_{0}}{\partial t}+\frac{\partial \phi_{7}}{\partial t}+\sum_{j=1}^{6} \frac{\partial \dot{s}_{j}}{\partial t} \varphi_{j}\right]
\end{aligned}
$$

Aus der Integration des Druckes über die benetzte Oberfläche ergibt sich die Kraft $\underline{F}_{h y d}$

$$
\begin{aligned}
\underline{F}_{h y d} & =\iint_{\bar{S}_{b}} p \underline{n}^{*} d S \\
& =-\rho \iint_{\bar{S}_{b}} \frac{\partial}{\partial t}\left(\phi_{0}+\phi_{7}\right) \underline{n}^{*} d S-\rho \sum_{j=1}^{6} \iint_{\bar{S}_{b}} \varphi_{j} \frac{\partial \dot{s}_{j}}{\partial t} \underline{n}^{*} d S
\end{aligned}
$$


Analog zu der Linearisierung der Randbedingung (C.4) werden hier auch nur kleine Wellen- und Bewegungsamplituden berücksichtigt. Somit ist die momentane benetzte Oberfläche näherungsweise gleich wie die mittlere benetzte Körperoberfläche, d.h. $S_{b} \approx \bar{S}_{b}$. Das erste Integral beinhaltet nur die Druckanteile aus dem Potential der Initialwelle und dem Diffraktionspotential $\phi_{0}+\phi_{7}=\varphi_{D} e^{i \omega t}$ und ergibt die von den Körperbewegungen unabhängigen Erregerkräfte. Dividiert man durch den Faktor $e^{i \omega t}$ und durch die Wellenamplitude $\zeta_{a}$, so ergibt sich der generalisierte Vektor der Erregerkraft $\underline{f}=\left(f_{1}, f_{2}, \ldots, f_{6}\right)^{T}$ :

$$
\underline{f}=\frac{i \omega \rho}{\zeta_{a}} \iint_{\bar{S}_{b}} \varphi_{D} \underline{n}^{*} d S
$$

Die Matrix der hydrodynamischen Massen $\underline{\mathcal{A}}=\left\{a_{l j}\right\}$ und die Matrix der Potentialdämpfungen $\underline{\mathcal{B}}=\left\{b_{l j}\right\}$ erhält man aus der Beziehung

$$
a_{l j}+\frac{i}{\omega} b_{l j}=-\rho \iint_{\bar{S}_{b}} \varphi_{l} n_{l}^{*} d S .
$$

Die Übertragungsfunktionen der Bewegung (RAO: Response Amplitude Operator) folgen aus den Newtonschen Bewegungsgleichungen:

$$
\left[-\omega^{2}(\underline{\mathcal{M}}+\underline{\mathcal{A}})+i \omega \underline{\mathcal{B}}+\underline{\mathcal{C}}\right] \frac{\underline{s}}{\zeta_{a}}=\underline{f}
$$

Die $[6 \times 6]$ Matrix $\underline{\mathcal{C}}$ enthält die hydrostatischen Rückstellkoeffizienten (siehe z.B. Newman,1977). 